\title{
Molecular Beam Scattering from Ultrathin Metallic Films
}

Dissertation

zur Erlangung des mathematisch-naturwissenschaftlichen Doktorgrades „Doctor rerum naturalium“ der Georg-August-Universität Göttingen im Promotionsprogramm Chemie der Georg-August-University School of Science (GAUSS)

vorgelegt von

Christoph Steinsiek

aus Rheda-Wiedenbrück

Göttingen, 2017 


\section{Betreuungsausschuss}

Alec Wodtke, Abteilung Wodtke, Institut für Physikalische Chemie, Institut für Physikalische Chemie / Max-Planck-Institut für biophysikalische Chemie

Dirk Schwarzer, Abteilung Wodtke, Institut für Physikalische Chemie, Institut für Physikalische Chemie / Max-Planck-Institut für biophysikalische Chemie

\section{Mitglieder der Prüfungskommission}

Referent: $\quad$ Prof. Alec Wodtke, Abteilung Wodtke, Institut für Physikalische Chemie / Max-PlanckInstitut für Biophysikalische Chemie

Co-Referent: Prof. Dirk Schwarzer, Abteilung Wodtke, Institut für Physikalische Chemie / MaxPlanck-Institut für Biophysikalische Chemie

Weitere Mitglieder der Prüfungskommission:

Prof. Jürgen Troe, Abteilung Troe, Institut für Physikalische Chemie

Prof. Jörg Schroeder, Abteilung Troe, Institut für Physikalische Chemie

Prof. Ricardo Mata, Abteilung Mata, Institut für Physikalische Chemie

PD Dr. Thomas Zeuch, Abteilung Suhm, Institut für Physikalische Chemie

Tag der mündlichen Prüfung:

07.07.2017 


\section{Content}

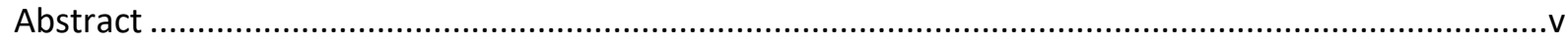

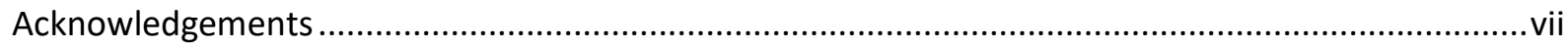

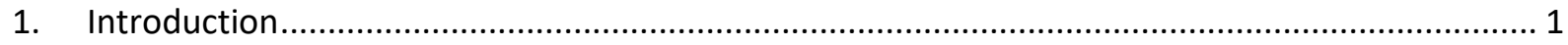

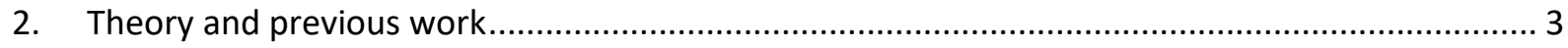

2.1 Validity of the Born-Oppenheimer Approximation at Metal Surfaces.............................. 3

2.2 Nonadiabatic Vibrational Energy Transfer in Molecule-Surface Collisions......................... 7

2.2.1 Molecular Vibrational Excitation .................................................................. 7

2.2.2 Molecular Vibrational Relaxation ................................................................. 11

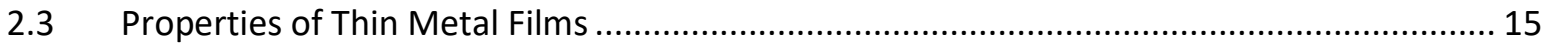

2.3.1 Epitaxial Growth Mode of Thin Metallic Film Systems........................................ 15

2.3.2 Electronic Surface Properties of Thin Metallic Films ............................................ 17

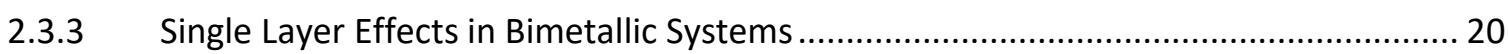

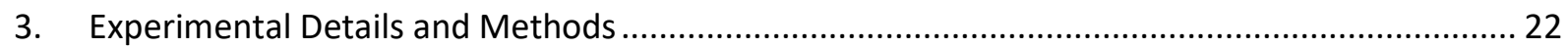

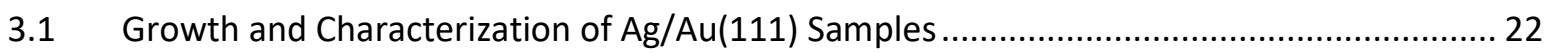

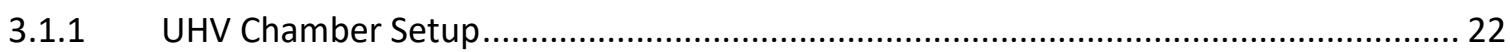

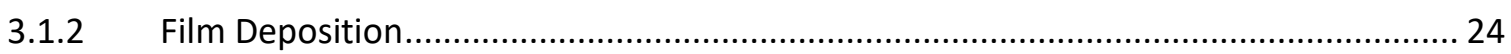

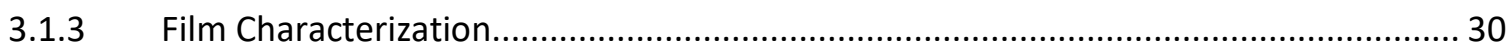

3.2 Molecular Beam Scattering Experiments ................................................................ 34

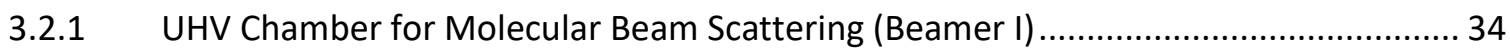

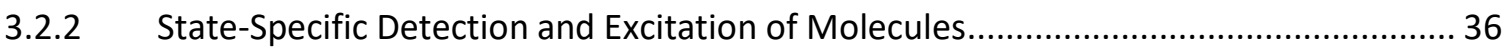

3.2.3 Beamer I: Wedge Sample Preparation and Position/Thickness Assignment ............... 38

3.2.4 Experimental Details for NO/CO translational energy transfer ............................... 40

3.2.5 Experimental Details for REMPI Signal Intensity Experiments...............................42

3.2.6 Experimental Details for Angular Distribution Measurements .............................. 47

4. Translational Inelasticity in Molecular Beam Scattering from Ultrathin Films of Ag/Au(111)..... 49

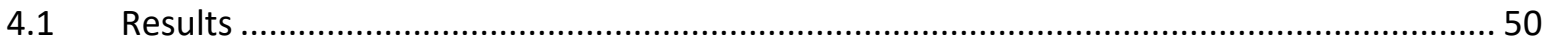

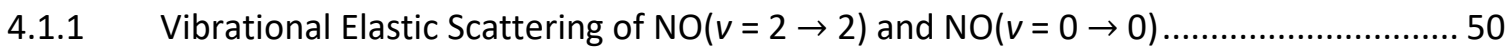

4.1.2 Inelastic $\mathrm{NO}(v=2)$ Scattering - Thickness Dependence $\mathrm{NO}(v=2 \rightarrow 1) \ldots \ldots \ldots \ldots \ldots \ldots . \ldots 3$

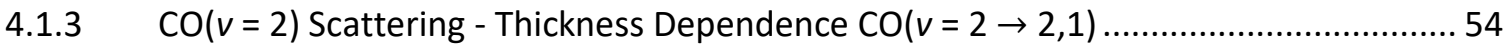

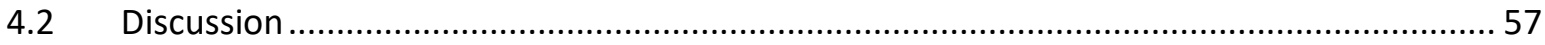

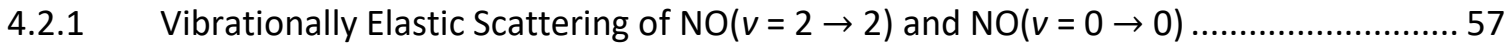

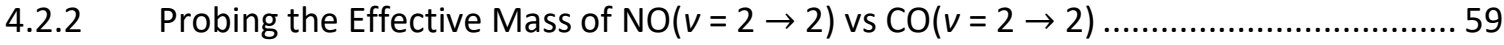

4.2.3 Vibration-Translation (V-T) Coupling of NO and CO on Au(111) and Ag/Au(111) .......60

4.2.4 A Possible Mechanism Explaining the Film Thickness Trend .............................63

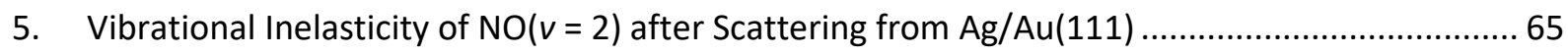




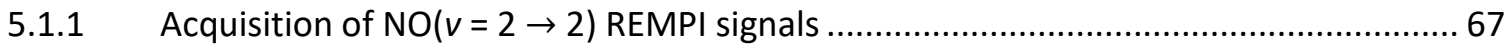

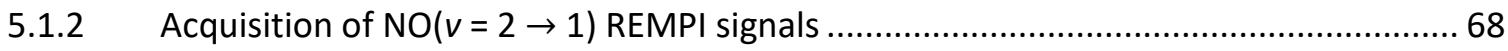

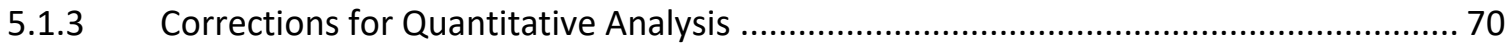

5.1.4 Determination of Absolute Relaxation Probabilities .......................................... 74

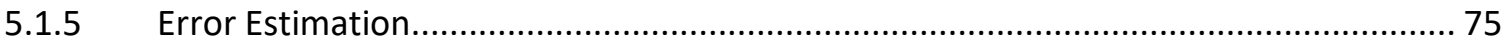

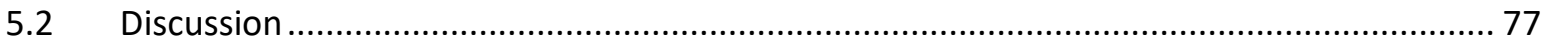

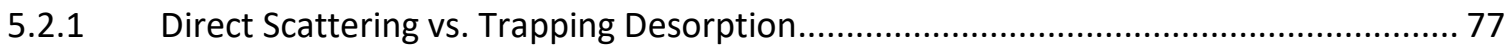

5.2.2 Role of Surface Characteristics for Vibrational Energy Transfer on Metal Surfaces ..... 78

5.2.3 Mechanistic Discussion: Influence of the Work Function on Vibrational Relaxation ... 82

5.2.4 Alternative Explanations for the Increased Relaxation Probability on $\mathrm{Ag} / \mathrm{Au}$..............85

5.2.5 A General Description of Vibrational Relaxation at Surfaces...................................8 86

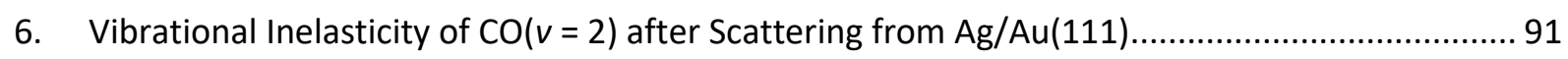

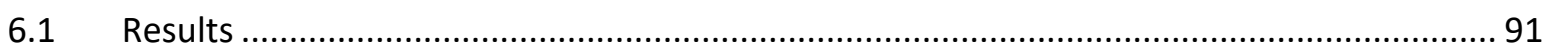

6.1.1 Acquisition of $\mathrm{CO}(v=2 \rightarrow 2)$ and $\mathrm{CO}(v=2 \rightarrow 1)$ REMPI Signals...............................91

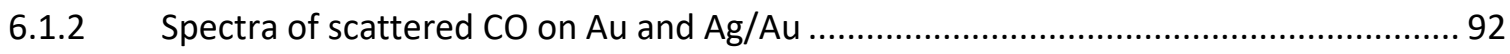

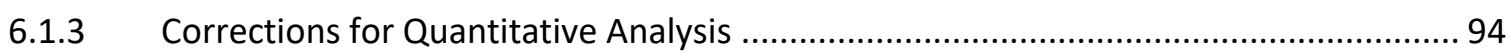

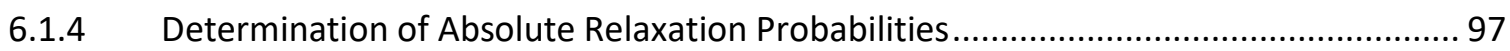

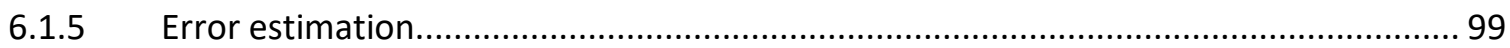

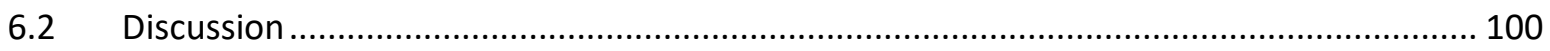

6.2.1 Direct Scattering vs. Trapping Desorption....................................................... 100

6.2.2 Vibrational Energy Transfer of $\mathrm{CO}(v=2)$ at Metal Surfaces.................................. 101

6.2.3 Mechanistic discussion: $\mathrm{CO}(v=2)$ relaxation at bulk (-like) surfaces....................... 103

6.2.4 $\mathrm{CO}(v=2)$ at $1 \mathrm{ML} \mathrm{Ag/Au}$ in the context of other relaxation measurements ............. 107

6.2.5 A special single layer effect for $\mathrm{CO}$ on $\mathrm{Ag} / \mathrm{Au}$ ? ................................................ 108

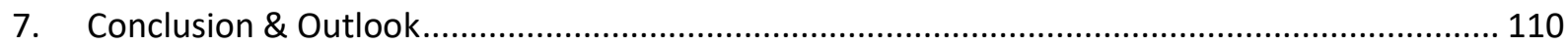

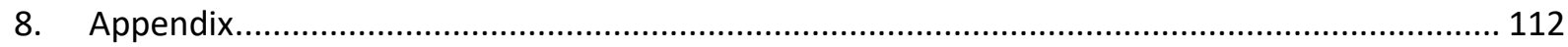

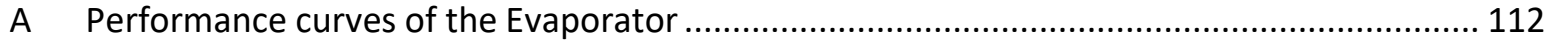

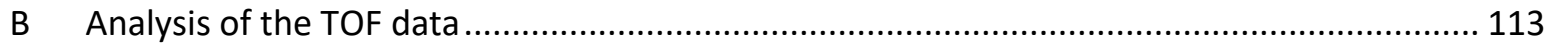

C Comparison $\mathrm{NO}(v=2 \rightarrow 2)$ and $\mathrm{NO}(v=0 \rightarrow 0)$ on $\mathrm{Au}$ and $\mathrm{Ag} / \mathrm{Au}$ with $\mathrm{NO}(v=0 \rightarrow 0)$ from bulk

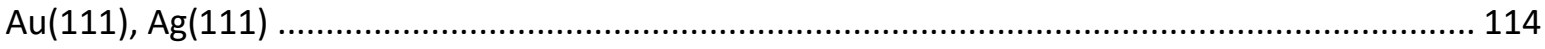

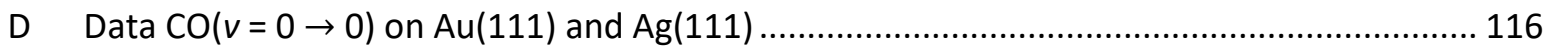

E Parameters for the model potential energy surface of NO/Au(111) and similar systems .... 117

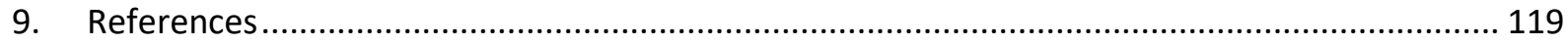

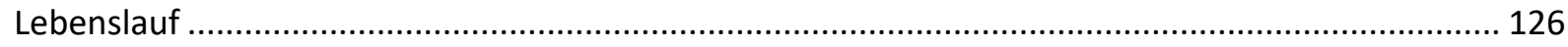




\section{Abstract}

One of the goals of studying molecule-surface interactions is to understand the details of the chemical reactivity at surfaces. The exchange of energy between a gas and a solid is a complex sequence of events even when focused on a model system like a diatomic molecule scattering from a monocrystalline metal surface. Due to the number of energy channels of the molecule (translational, rotational and vibrational energy) and the surface (phonons, electrons) involved, a detailed knowledge about the role of each of these degrees of freedom in the collision is necessary for the modeling of such processes. Over the last decades, the importance of electronically nonadiabatic interactions in molecule-surface interactions, where the Born-Oppenheimer Approximation is not valid anymore, has become clear. New theoretical models going beyond this approximation are needed to adequately describe such processes. Equally important is the acquisition of experimental data of model systems as a standard for comparison that can challenge the validity of such models. Although many ideas and concepts could be learned during the investigation of flat single crystals, "real" catalysts used in industrial processes are substances complex in structure and chemical composition. The introduction of model systems with a higher degree of complexity like bimetallic layers in the field of surface science bridges the gap between the ideal behavior of models and the diversity of "real world" catalysts.

The present work investigates the role of the surface in molecule-surface scattering processes. In contrast to prior studies employing a single material surface, layers of $\mathrm{Ag} / \mathrm{Au}(111)$ with defined thickness are chosen as a target. Beam scattering methods, combined with laser-based detection techniques, are used to investigate the molecules NO and CO with many similar properties but different strength of nonadiabatic coupling.

We examined translational energy exchange of vibrationally excited $\mathrm{NO}(v=2)$ and $\mathrm{CO}(v=2)$ from ultrathin metallic films of $\mathrm{Ag} / \mathrm{Au}(111)$ as a function of film thickness at an incidence translational energy of $0.6 \mathrm{eV}$. For NO, arrival time distributions were measured for vibrationally elastic $(v=2 \rightarrow 2$ and $v=0 \rightarrow 0)$ and inelastic scattering $(v=2 \rightarrow 1)$ for Ag film thicknesses up to $33 \mathrm{ML}$. For CO, vibrationally elastic $(v=2 \rightarrow 2)$ and inelastic $(v=2 \rightarrow 1)$ scattering channels were examined in a similar manner. For both molecules and for all investigated vibrational states, we observe a gradual decrease of the mean final translational energy for $0-3 \mathrm{ML} \mathrm{Ag} / \mathrm{Au}$ films before reaching constant behavior for higher thicknesses (3-30 ML). The results are consistent with experimental data for pure $\mathrm{Au}$ and $\mathrm{Ag}$ crystal surfaces, and can be explained as head-on elastic collisions between NO molecules and surface elements with effective masses of 200amu $\left(1.0 \mathrm{~m}_{\mathrm{Au}}\right)$ and $130 \mathrm{amu}\left(1.2 \mathrm{~m}_{\mathrm{Ag}}\right)$. Due to the substantial change of translational inelasticity up to a film thickness of $3 \mathrm{ML}$, we propose that subsurface layers take part in the scattering process. The thickness dependence of the final translations energy suggests that this kind of inelasticity is purely dominated by mechanical properties of a surface.

Furthermore, we measured the thickness-dependent relaxation probabilities of $\mathrm{NO}(v=2)$ and $\mathrm{CO}(v=2)$ scattered off $\mathrm{Ag} / \mathrm{Au}(111)$. Both molecules show a significantly different trend. For $\mathrm{NO}(v=2)$, which is characterized as a molecule with strong nonadiabatic coupling strength, relaxation is gradually increasing between 0-3 ML $\mathrm{Ag} / \mathrm{Au}$ until reaching a constant value, dominated by multiquantum relaxation into the vibrational ground state. A linear correlation between the survival probability of $\mathrm{NO}(v=2)$ and the work function strongly suggests that this property determines the amount of vibrational energy loss during the collision event. The data is discussed in the context of previous relaxation experiments exhibiting a trend when plotting the relaxation probability against the difference of the vertical electron binding energy (VEBE) of a vibrationally excited molecule and the work function of a bulk surface. For $\operatorname{CO}(v=2)$ which is understood as a molecule with only weak 
nonadiabatic coupling, scattering from $\mathrm{Au}(111)$ and films above $3 \mathrm{ML} \mathrm{Ag} / \mathrm{Au}(111)$ exhibits similar low relaxation probabilities. However, for a thickness of $1 \mathrm{ML} \mathrm{Ag} / \mathrm{Au}$, a maximum of vibrational relaxation into the ground state is observed. The equal amount of energy loss when scattered from $\mathrm{Au}(111)$ and $\mathrm{Ag}(111)$-like surfaces shows that in the case of $\mathrm{CO}$, the surface work function does not substantially influence vibrational relaxation, probably due to the low electron affinity of $\mathrm{CO}(-1.5 \mathrm{eV})$ in comparison to $\mathrm{NO}(0.026 \mathrm{eV})$. The peaking of $\mathrm{CO}(v=2)$ relaxation at $1 \mathrm{ML} \mathrm{Ag} / \mathrm{Au}$ cannot be assigned to a single surface property with a maximum/minimum emerging for this film thickness. Moreover, the dependence of vibrational relaxation does not follow the trend of the previously suggested model on the basis of VEBE and surface work function. It is therefore interpreted as a special effect characteristic for a bimetallic layer system as observed previously for many metal-metal bilayer systems. Possible effects that explain the high relaxation probability are discussed. 


\section{Acknowledgements}

First, I would like to thank Alec Wodtke for the opportunity to work in his group. You gave me a lot of support, motivation and were an inspiration for many ideas over the last several years. Thanks to your both encouraging and skeptic attitude, I could learn a lot about science and I really enjoyed working in your group!

Next, I thank Christof Bartels for his supervision and guidance since the beginning of the thin film project. You taught me a lot when we set up the test chamber and provided me with advice when I had no clue how to solve experimental problems. Furthermore, thanks a lot for your feedback in all scientific discussions.

I want to say thank you to Pranav Shirhatti. It was a pleasure to work with you in the laboratory measuring with and sometimes fighting against the machine. Devices kept failing but thanks to you we kept on solving puzzles experimentally and scientifically which was a lot of fun.

I would like to thank Dirk Schwarzer who helped me especially in the beginning when I started in this group. He designed many excellent devices (like the evaporator) that contributed substantially in the projects that I was working on.

There were many people working together with me in the lab making the work even more fun. Jan Geweke, Arthur Meling and Sven Meyer, I know that your will treat the good old "Beamer I" setup well and I wish you good luck with your experiments!

A special thanks to all the people that I had fruitful discussions about the results of the relaxation experiments, Bastian Krüger, Roman Wagner, Tim Schäfer. Bastian and Roman contributed data and scientific input to find the "Komplettlösung" of vibrational relaxation. Furthermore I have to thank Sascha Kandratsenka and Jan Altschäffel for the explanations and discussions regarding the underlying theory.

Thank you Dan Harding and Theo Kitsupoulos for letting me use the Auger device for the characterization of the thin films at the test chamber.

I have to acknowledge the help and advice from the people from both mechanical and workshop of the Institut für Physikalische Chemie. Without people like you it would not have been possible for me to set up any new experiments. Special thanks to Volker Meyer, Reinhard Hildebrandt and Andreas Knorr.

I really enjoyed working in the Wodtke group with all you nice people who provided a good atmosphere. Great thanks to everybody!

Auch möchte ich meiner Familie danken, meinen Eltern und meiner Schwester, die mich immer unterstützt haben.

最後に、伊藤麻衣さんに感謝の気持ちを伝えたいと思います。時には実験や論文執筆の際に 迷惑をかけたこともありましたが、色々と支えてくれて本当にありがとうございました。 


\section{Introduction}

Numerous applications of heterogeneous catalysis are widespread in modern societies and have a profound influence on our daily lives. Examples include, the removal of toxic gases from the exhaust of internal combustion engines in addition to a large number of industrial processes, such as the fixation of nitrogen in ammonia synthesis [1].

For a detailed understanding of heterogeneous catalytic processes, it is crucial to gain insights into the fundamental steps of energy transfer during a molecule-surface interaction [2]. The complexity arises as early as the first step, in which the molecule collides with the surface. A molecule carrying energy in translational, rotational, vibrational and electronic degrees of freedom impinges on the surface. During the collision, it might gain or lose energy by interaction with the lattice vibrations or electronhole pairs of the surface. In addition to direct scattering which involves a single collision event with a short interaction time, the molecule might be trapped temporarily or permanently. The diversity of scattering processes reflects the complexity of the dynamics of gas-surface interactions. With the ultimate goal of controlling chemical reactivity for the design of better and more efficient catalysts, it is important to understand quantitatively which energy channels are participating in a gas-surface collision step and how energy conversion can be controlled.

Energy transfer in gas-surface collisions is best studied in non-reactive molecule-surface systems and it has proven to be successful to choose a bottom-up strategy that concentrates on simple model systems. Experimentally, molecular beam scattering plays an important role in the exploration of energy-transfer dynamics. Pioneering works have focused on diatomic molecules scattered from single crystal surfaces and investigated the dependence of the final quantum state distribution of a molecule on the initial conditions [3-7]. These state-to-state scattering measurements were performed using molecular beams in combination with laser-based techniques. Supersonic expansion from a nozzle produces rotationally cold molecular beams that can be excited to desired vibrational states by intense infrared laser pulses. Final quantum state distributions after the surface collision can be measured by state-selective laser detection techniques. Performing measurements on clean low index metal surfaces under ultra-high vacuum (UHV) conditions minimizes changes of the surface in the course of the experiment. Additionally, the choice of simple model systems makes it possible to compare the experimental results to first-principles theory.

A number of basic ideas and concepts can be extracted by studies of single crystal surfaces. However, the application of those insights might be limited in regard to real catalysts that are used in industrial processes. In contrast to simple and ordered surfaces, "real" catalysts are often much more complex in structure and chemical composition [8]. One step toward bridging this "material gap", as this problem is called in the field of surface science, is to increase complexity by the introduction of metal films as model systems. Although there are already many thorough investigations of the growth and structural properties of bimetallic layer systems [9] and a number of systematic experimental and theoretical studies about their electronic [10-12] and chemical properties [13], there have been no studies about molecular beam scattering studies focusing on molecular energy transfer and its dependence on the film thickness.

The present work is about understanding the energy transfer of $\mathrm{NO}, \mathrm{CO}$ with thin films of $\mathrm{Ag} / \mathrm{Au}(111)$ using scattering of $\mathrm{NO}(v=2)$ and $\mathrm{CO}(v=2) . \mathrm{Ag} / \mathrm{Au}(111)$ provides a nice model system for scattering experiments because it is relatively simple and has been well characterized. The lattice mismatch between $\mathrm{Au}$ and $\mathrm{Ag}$ is negligible providing a bilayer system with ideal layer-by-layer growth avoiding complicating effects like surface strain or strong local inhomogeneities at the surface. Several studies 
have investigated the electronic properties of $\mathrm{Ag} / \mathrm{Au}(111)$ films and their dependence on the film thickness. Moreover, both bulk materials have been subject to previous molecular beam scattering studies. The results in the present work can therefore be interpreted in the light of the surface properties and compared to the behavior from bulk samples. Finally, the insights obtained from the model system $\mathrm{Ag} / \mathrm{Au}(111)$ may be transferrable to other thin film systems.

With the probe molecules $\mathrm{NO}$ and $\mathrm{CO}$, two molecules with comparable molecular mass, dipole moment and vibrational frequency but very different electron affinity are chosen. The main focus is on the translational and vibrational energy transfer of the vibrationally excited species $\mathrm{NO}(v=2)$ and $\mathrm{CO}(v=2)$ and how it changes with film thickness. Vibrational relaxation is of particular interest. At metal surfaces, this process is understood as a nonadiabatic coupling between the molecular vibrational degrees of freedom with electron-hole pairs of the surface. Note that this energy transfer cannot be explained within the framework of the Born-Oppenheimer approximation (BOA). Determining the degree of vibrational relaxation can therefore be a measure to quantify the breakdown of this important approximation. Recent state-of-the-art theoretical descriptions struggle to describe vibrational relaxation correctly on a quantitative level [14], it is therefore hoped that the results of this work can act as benchmark for new or improved existing theoretical models. 


\section{Theory and previous work}

This section will provide a theoretical background about concepts important in the context of the present work. First, a short introduction about nonadiabatic effects will be given, followed by a discussion of previous experimental work on vibrational energy transfer. The final paragraph will be about growth and properties of thin metals with special focus on the $\mathrm{Ag} / \mathrm{Au}(111)$ system employed in the present work.

\subsection{Validity of the Born-Oppenheimer Approximation at Metal Surfaces}

Due to its fundamental role in theoretical surface science, the breakdown of the Born-Oppenheimer approximation at surfaces is topic of fundamental importance for the calculation of potential energy surfaces and is treated extensively in the literature. A brief overview will be provided blow, the interested reader might be directed to references [15-18].

The potential energy surface (PES) is a fundamental concept in chemistry containing all the atomic scale forces that control a reaction depending on the nuclear arrangement. Not only does it offer a mathematical description that defines the base for trajectory calculations, it also allows the visualization of a reaction and delivers with its peaks and valleys an intuitive approach to understand which molecular configurations are stable.

The PES is typically defined within the Born-Oppenheimer approximation (BOA) which takes note of the great difference between the masses of nuclei and electrons assuming that the electrons respond instantaneously to displacement of the nuclei [19]. Within that approximation, it is possible to solve the Schrödinger equation for the electrons in a static potential with a fixed nuclear geometry yielding the energy for this particular configuration. By repeating the calculation for a number of arrangements of the nuclei, the PES can be constructed. In principle, for every electronic state, a separate PES can be obtained but in most cases, the PES corresponding to the ground electronic state is of interest. Because within the BOA, the nuclei remain on a single adiabatic PES, this approach is also termed as the adiabatic approximation. While at the time of its introduction, the BOA allowed for the first time to calculate potentials which were based on the quantum theory, nowadays it is still the fundament of theoretical chemistry.

However, there are certain requirements that have to be met to ensure the validity of this approximation: On the one hand, (1) electrons have to adjust immediately with the change of the nuclei positions. This condition might be violated either in the case of strong coupling between both electronic and nuclear motion or a weak coupling but high velocity of the nuclei. On the other hand, (2) different electronic PESs have to be widely separated in energy. For many gas-phase reactions, adiabatic models have had great success because in most cases, nuclei velocities are sufficiently slow that instantaneous adjustment of the electrons is possible and energy levels are far apart due to the quantized nature of molecular electronic states.

At metal surfaces, however, violations might occur more often than in gas phase. Here, the barrier for electron transfer processes is substantially decreased because the ionization energy of transition metal 
surfaces (which the work function) is typically in the order of $5 \mathrm{eV}$ while simple diatomic molecules $\left(\mathrm{H}_{2}\right.$, $\mathrm{NO}, \mathrm{CO}, \mathrm{N}_{2}$ ) exhibit values in the order of $10 \mathrm{eV}$. Furthermore, the formation of ions close to a metal surface is energetically stabilized due to an image potential. As a result, an avoided crossing of the ionic and the neutral potential of a molecule might arise for which the BOA is not valid anymore and nonadiabatic energy transfer is possible. An example is the electron transfer to a (vibrationally excited) NO molecule scattering from a metal surface. This situation is sketched in Figure 2.1 in a simplified manner, showing the neutral and one anionic potential energy curve as a function of surface-distance $z$. At large distance to the surface, the neutral state of molecule $A$ is energetically more stable in comparison to the anionic state. However, due to the image charge stabilization, the anionic potential is lowered in energy in closer distance, leading to an avoided crossing (red circle at $z=2 \AA$ ). At those points, the decoupling between electronic and nuclear motions is not possible and the BOA is not valid anymore.

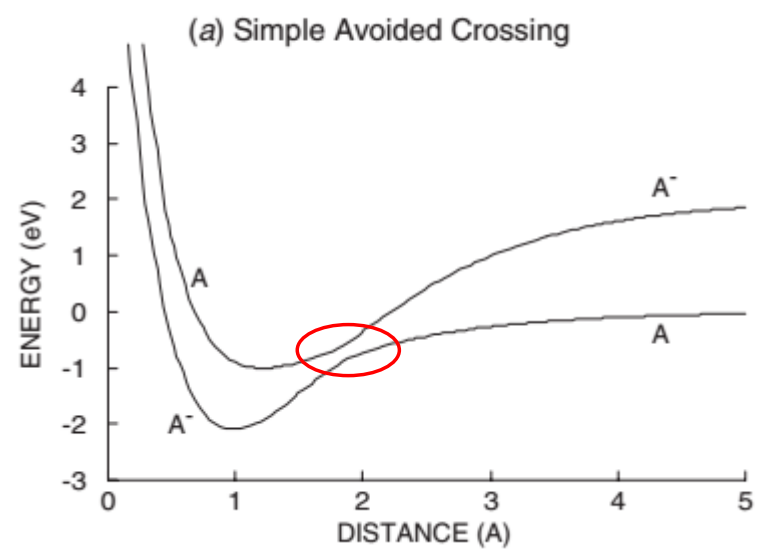

Figure 2.1: Simplified model of the potential energy curves in the case of an electron transfer for the example of NO/Au(111). At the avoided crossing (red circle), the BOA is not valid anymore. Taken from Ref. [15].

The situation is further complicated by the presence of a continuum of electronic states at the surface. Depending on the energy level of the electron participating in the electron transfer, the anionic level is different in energy resulting in a continuum of possible anionic potential energy curves. Again, one requirement for the BOA, a wide separation of the states, is not fulfilled anymore. This situation is depicted in Figure 2.2 (left panel). The lowest curve of the continuum represents the interaction with an electron directly at the Fermi level, while electrons that are more strongly bound will result in curves shifted by corresponding offset to the Fermi level.
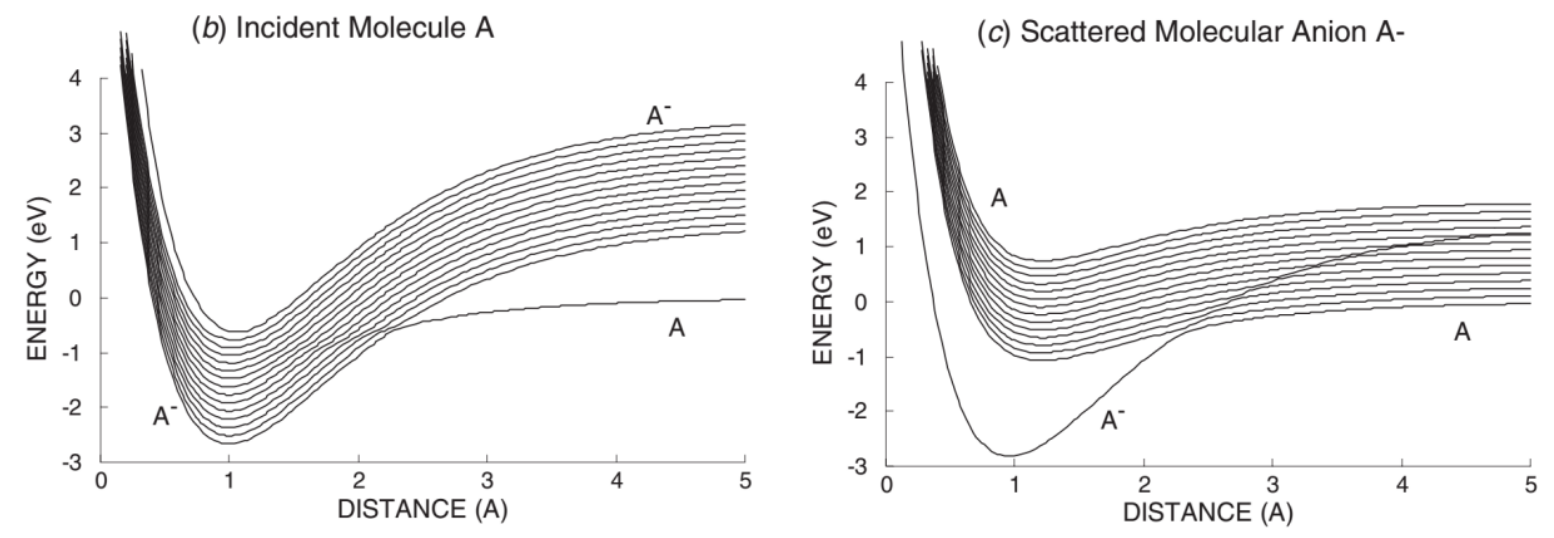

Figure 2.2: Continuum of potential energy curves arising due to the continuum of electronic levels at the metal surface. Left: Continuum of possible anionic states for the approaching molecule A. Right: Continuum of possible neutral states for the anion $A^{-}$when receding from the surface. Taken from Ref. [15]. 
A similar situation arises when the electron is transferred to back. After collision and receding as an anion from the surface, the avoided crossing is approached again. Because of the high number of available states with different energies that can accept the electron, a continuum of neutral states is possible as depicted in Figure 2.2 (right panel). Note that the existence of the anion $A^{-}$is purely transient at distances close to the surface where it energetically favored.

Now, it could be imagined that the neutral molecule approaches and leaves the surfaces forming the transient ion in an adiabatic process. In that case, the electron would be removed from the Fermi level of the metal and returned exactly to the Fermi level following the electronic adiabatic ground state. However, due to the amount of nonadiabatic pathways that are possible it is likely that a high amount of electron transfer processes lead to a net excitation of electron-hole pairs. The electron transfer can be seen as a tunneling process that happens with a limited probability. Especially fast molecules will have approached that surface closer than the avoided crossing region undergoing a nonadiabatic process. To conclude, the stabilization of charged particles and the existence of a continuum of states at metal surfaces lead to a situation where nonadiabatic transfer processes are highly likely to occur.

Experimentally, there are many well-documented examples of the breakdown of the BOA in molecular processes at surfaces. Early findings include phenomena like chemiluminescence [20], electron and anion emission during the reaction of low work function surfaces with of $\mathrm{O}_{2}$ [21] or halogens [22]. That electronically nonadiabatic effects can play an important role for the interaction between electronic degrees of freedom of a metal surface and molecular vibrations was observed when comparing the vibrational lifetime of $\mathrm{CO}$ adsorbed on metals and on an insulator surface. While vibrationally excited $\mathrm{CO}$ was found to have relaxed within $2 \mathrm{ps}$ when adsorbed on a $\mathrm{Cu}(100)$ surface [23], the lifetime of $\mathrm{CO} / \mathrm{NaCl}$ was on the ms-scale close to its gas phase value [24]. The continuum of electronic states present at $\mathrm{Cu}(100)$ leads to vibrational quenching of the molecules, a pathway which is not available at an insulating surface.

The main topic of the present work is vibrational energy transfer during molecular beam scattering. In contrast to the previously introduced example, molecules are not in an adsorbed state but scatter from the surface experiencing only a short interaction in the sub-ps time scale. Before elaborating on this topic, two rather recent examples of nonadiabatic processes shall be highlighted.
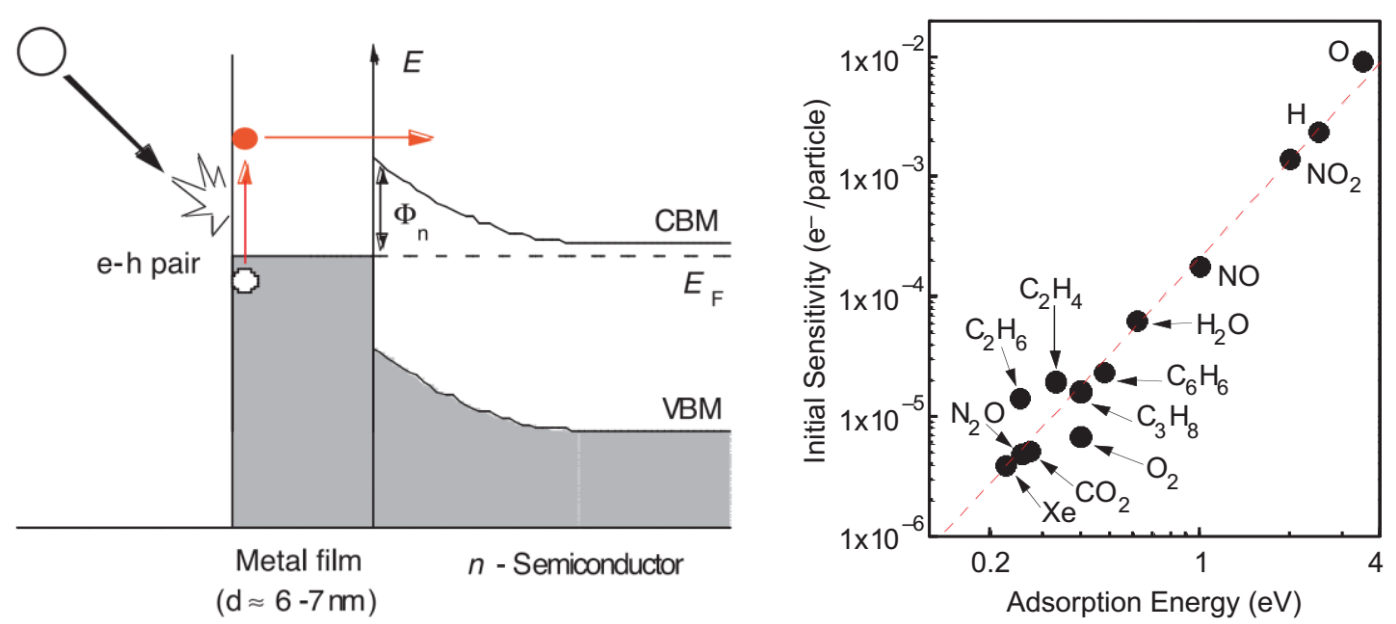

Figure 2.3: Chemicurrents. Left: Excitation of electron-hole pairs at the surface by chemical reaction, leading to a measurable current. Right: Correlation between the electron detection sensitivity and the adsorption energy. Taken from Ref. [25].

The first is the detection of chemically induced electronic excitations at metal surfaces, also referred to as "chemicurrents" [25]. Schottky diodes consisting of a silver film of a few nm thickness were grown on Si(111). After exposition to a number of different atomic and molecular gas species (Figure 2.3, left 
panel) a measurable current signal could be detected at the diode. The initial sensitivity of the electron signal correlated very strongly with the adsorption energy of the molecule (Figure 2.3, right panel).

The current understanding of this effect is that the energy released by the surface reaction leads to electron-hole-pair excitations that can be directly detected as an electronic signal. It was shown that the phenomenon is observed for different metal and semiconductor structures [26]. Those findings suggest that electronic excitations and therefore nonadiabatic effects are a common feature for chemical reactions at metal surfaces.

A second example shows the importance of nonadiabatic effects for the very basic interaction between $\mathrm{H}$ atoms and a metal surface. The question why $\mathrm{H}$ atoms can stick efficiently to a metal surface has been a mystery for a long time. In a collision with a $\mathrm{Au}(111)$ surface, those particles are expected to lose only $2 \%$ of its initial energy in a mechanical picture according to energy and momentum conservation. A high resolution time-of-flight scattering experiment with $\mathrm{H}$ atoms could show that nonadiabatic effects have a strong influence on the energy exchange with the surface [27]. Nearly monoenergetic beams of $\mathrm{H}$ atoms were produced via laser photolysis and scattered off a $\mathrm{Au}(111)$ surface. Directly after the collision, the atoms were tagged via laser excitation into a high Rydberg state. After traveling through a defined field-free region, strong field ionization and detection made it possible to measure the translational energy distribution. Instead of a metallic $\mathrm{Au}(111)$ surface, an insulating surface of solid Xe could be produced by cooling the surface down in an atmosphere of Xe.

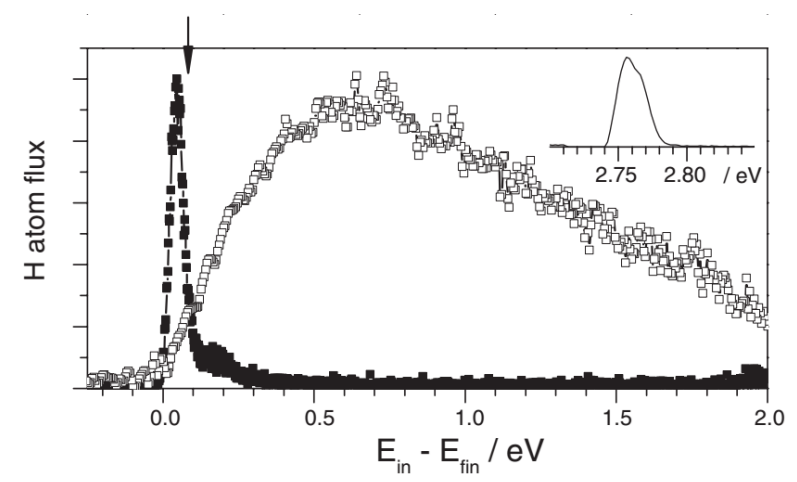

Figure 2.4: Kinetic energy loss spectrum of $\mathrm{H}$ after scattering from solid Xe (black squares) and from Au(111) (white squares). The inset shows the kinetic energy distribution of the incoming $\mathrm{H}$ atom beam. The expected energy loss from a binary collision model is marked by a vertical arrow. Taken from Ref [27].

Figure 2.4 shows the energy loss spectrum of the $\mathrm{H}$ atoms when scattered off solid Xe (black squares) and $\mathrm{Au}(111)$ (open squares). After scattering from a Xe surface the $\mathrm{H}$ atoms exhibit a very narrow kinetic energy distribution as expected from a binary collision model (vertical arrow). Energy exchange is only feasible via interaction with phonons. In contrast, when scattering from $\mathrm{Au}(111)$ a broad distribution with an average loss of $0.9 \mathrm{eV}$ is obtained. The reason for this striking difference the metallic character of the $\mathrm{Au}(111)$ surface allowing the pathway of energy loss via electron-hole pair excitations. A theoretical model taking that model into account could reproduce the obtained spectrum. To conclude, those two examples provide an insight how widespread the nonadiabatic effects are in gas-surface reactions. 


\subsection{Nonadiabatic Vibrational Energy Transfer in Molecule- Surface Collisions}

One question of the present work is which characteristics of a molecule-surface system influence the effect of vibrational relaxation. Vibrational relaxation describes the process when a vibrationally excited molecule approaches a surface and collides while losing vibrational energy to the surface. This is typically a nonadiabatic process if the main energy loss channel is the interaction with electron-hole pairs of the surface. Historically, the first reported case of nonadiabatic vibrational energy transfer was the opposite but mechanistically related process of vibrational excitation. In that case, molecules in the ground state were observed to exhibit vibrational excitation upon scattering from a hot metal surface.

Currently, it is believed that the interaction of a gas-surface system can be characterized by a certain nonadiabatic coupling strength. Experimental evidence supports that a system with stronger nonadiabatic coupling (like NO/Au [28]) exhibits a higher probability for both vibrational excitation and relaxation in comparison to a system with relatively weaker coupling (like CO/Au [29]). To learn about the characteristics of nonadiabatic coupling, the following paragraph covers therefore both processes.

\subsubsection{Molecular Vibrational Excitation}

The first observed example of nonadiabatic vibrational excitation in molecule-surface collision was the observation of $\mathrm{NO}(v=1)$ after scattering of $\mathrm{NO}(v=0)$ from a hot $\mathrm{Ag}(111)$ surface by Rettner et al. [30]. For the excitation probability, two experimental signatures were found that are typical for electronically nonadiabatic interactions: (1) a dependence on the surface temperature $T_{\mathrm{s}}$ (Figure 2.5a). (2) A strong dependence on incidence energy $E_{\mathrm{i}}$ following a nonlinear trend in absence of a threshold (Figure 2.5b). When plotting the $T_{\mathrm{s}}$ dependence as shown in (Figure 2.5a) the data can be fitted with an Arrhenius-like function according to (2.1). While the activation energy is equal to the vibrational energy $\Delta E_{\mathrm{vib}}$, the pre-exponential factor $A$ can be used as a measure to describe to the nonadiabatic coupling strength.

$$
P_{\mathrm{NO}(v=0 \rightarrow 1)}=A \exp \left(-\frac{\Delta E_{\mathrm{vib}}}{k_{\mathrm{B}} T_{\mathrm{S}}}\right)
$$

Those results were interpreted according to a mechanism by which the NO molecule is excited due to energy gain from thermally excited electron-hole pairs of the metals surface. A theoretical model by Newns based on that mechanism was able to reproduce the results [31] supporting concept of interaction between molecular degrees and electron-hole pairs.

However, vibrational excitation can follow a different mechanism as well. $A$ behavior in contrast to the previous example was found for the vibrational excitation of $\mathrm{NH}_{3}$ scattered from $\mathrm{Au}(111)$ [32] which is regarded as an electronically adiabatic process. As shown in Figure 2.5c for an incidence energy of $\sim 0.3 \mathrm{eV}$, the probability to find the scattered molecule in the $v_{2}$ ("umbrella") mode was independent of the surface temperature $T_{s}$. The dependence of the excitation probability on incidence energy follows a linear trend with a threshold close to the vibrational energy. Below that energy, no excitation is observed indicating direction conversion of incidence translational energy to molecular vibration. 
The presented examples of $\mathrm{NO}(v=0 \rightarrow 1) / \mathrm{Ag}(111)$ and $\mathrm{NH}_{3}(v=0 \rightarrow 1,2,3) / \mathrm{Au}(111)$ are two limiting cases. It should be noted that a mixed mechanism could be found like in the example of $\mathrm{HCl}(v=0 \rightarrow 1) / \mathrm{Au}$ [33]. Here, for lower surface temperatures $T_{\mathrm{s}}<800 \mathrm{~K}$, the excitation probability can be observed but remains constant as described for an adiabatic mechanism but increases for higher temperature for which nonadiabatic excitation dominates.

There are now a number of reported examples about nonadiabatic vibrational excitation for several molecule-surface systems that allow conclusions about which parameters determine the nonadiabatic coupling strength. Based on those observations we might learn which characteristics of a surface or a molecule determine the amount of "nonadiabaticity", that a prediction of the tendency to undergo processes like vibrational excitation or relaxation is possible.

The influence of a surface on nonadiabatic coupling strength might obtained by comparing the excitation probability $\mathrm{NO}(v=0 \rightarrow 1)$ that was measured for different surfaces. Experimental data is available for the surfaces of $\mathrm{Ag}(111)$ [30], $\mathrm{Cu}(110)$ [34] and $\mathrm{Au}(111)$ [35]. Because those experiments were carried out under different conditions, $E_{\mathrm{i}}$ dependence of the excitation probability was selected at a common surface temperature and is depicted in Figure 2.6 (left panel). It is difficult to measure absolute excitation probabilities and previous reviewers pointed out that for $\mathrm{Ag}(111)$ and $\mathrm{Cu}(110)$ the Arrhenius analysis according to equation (2.1) yields unrealistic pre-exponential factors, nevertheless it should be possible to estimate qualitative trends.
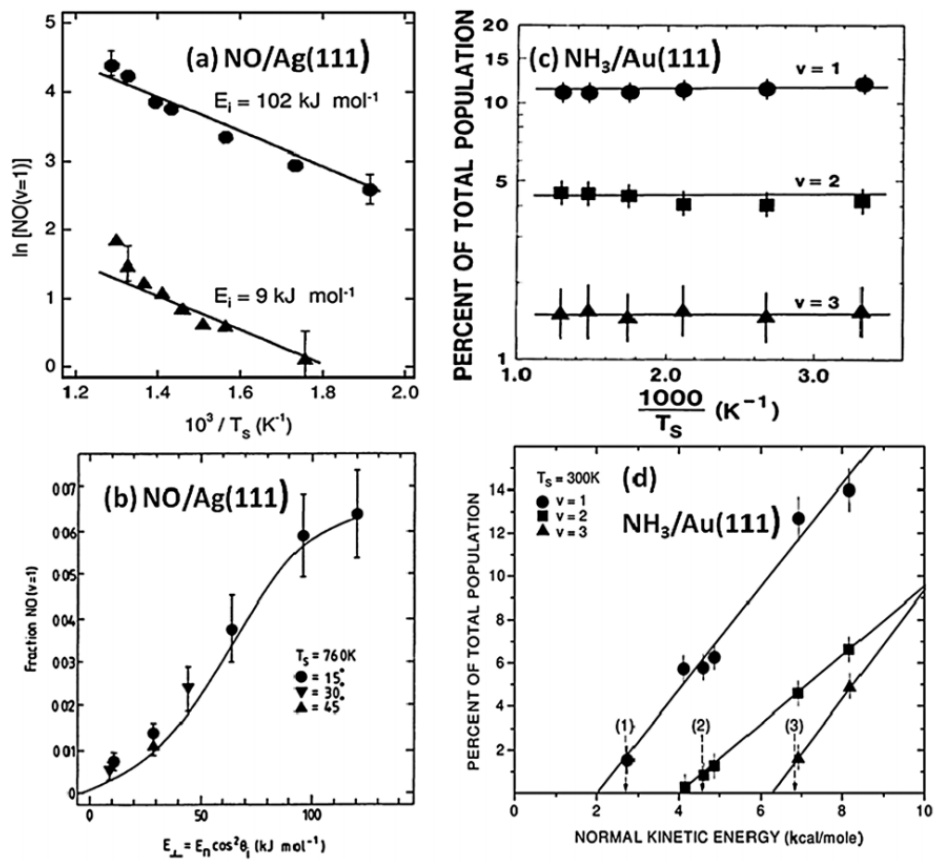

Figure 2.5: Comparison between nonadiabatic $(a, b)$ and adiabatic $(c, d)$ vibrational excitation: $T_{s}$ and $E_{i}$ dependence of the vibrational excitation probability $P_{N O(v=0 \rightarrow 1)}(a)$ : Nonadiabatic: $T_{s}$ dependence exhibiting Arrhenius behavior for the example of $\mathrm{NO}(v=0 \rightarrow 1) / A g(111)$. (b) $P_{N O(v=0 \rightarrow 1)}$ exhibits strong nonlinear $E_{i}$ dependence without a threshold. [30] (c) Adiabatic: Excitation probability of $\mathrm{NH}_{3}(v=0 \rightarrow 1,2,3) / \mathrm{Au}(111)$ is independent of $T_{s .}$ (d) $P_{N O(v=0 \rightarrow 1)}$ showing a linear behavior with clear threshold defined by the vibrational energy. [32] Taken from Ref. [18]. 

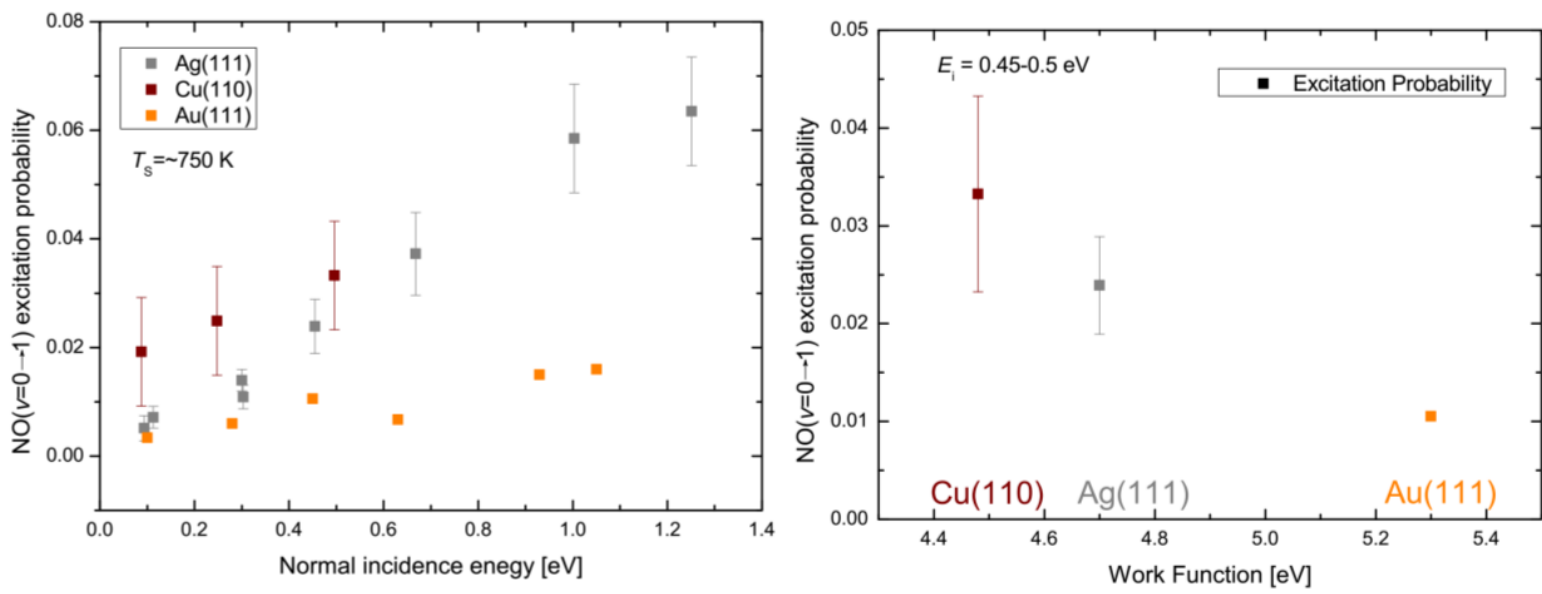

Figure 2.6: $\mathrm{NO}(v=0 \rightarrow 1)$ excitation probability at different surfaces. Left: Data at a common $T_{s}$ for $\mathrm{Ag}(111)[30], \mathrm{Cu}(110)$ [34] and $\mathrm{Au}(111)$ [35]. Right: Excitation probabilities for incidence energies of 0.45-0.5 eV plotted against the work functions of $\mathrm{Cu}(110)$ [36], $\mathrm{Ag}(111)$ [37], $\mathrm{Au}(111)$ [38].

The most obvious result is probably that $\mathrm{Ag}(111)$ and $\mathrm{Cu}(110)$ show a similar tendency for excitation, whereas the probability measured at $\mathrm{Au}(111)$ is significantly lower. This trend was first observed by Rahinov et al. [18] who suggested the work function $\phi$ as an "intuitive proxy" to quantify nonadiabatic interactions. Since excitation proceeds via the formation of a transient ion, lower work function materials are expected to show higher nonadiabatic coupling as predicted by the model by Newns [31]. The data of the three surfaces is in accord with that idea as it is shown in Figure 2.6 (right panel). For comparable incidence energies, the strongest excitation is observed on $\mathrm{Cu}(110)(\phi: 4.48 \mathrm{eV}[36])$, similar to $\mathrm{Ag}(111)$ ( $\phi: 4.72 \mathrm{eV}$ [37]) and the lowest tendency on $\mathrm{Au}(111)(\phi: 5.31 \mathrm{eV}$ [38]).

A second surface effect on the nonadiabatic coupling strength was experimentally found when measuring the $\mathrm{N}_{2}(v=0 \rightarrow 1)$ excitation probability after scattering from $\mathrm{Au}(111)$ and $\mathrm{Pt}(111)$ [39]. Due to the high work function of $\mathrm{Pt}(111)$ (5.8 eV) in comparison to $\mathrm{Au}(111)$, one would expect a lower tendency to undergo vibrational excitation on this surface. However, the opposite trend was found.
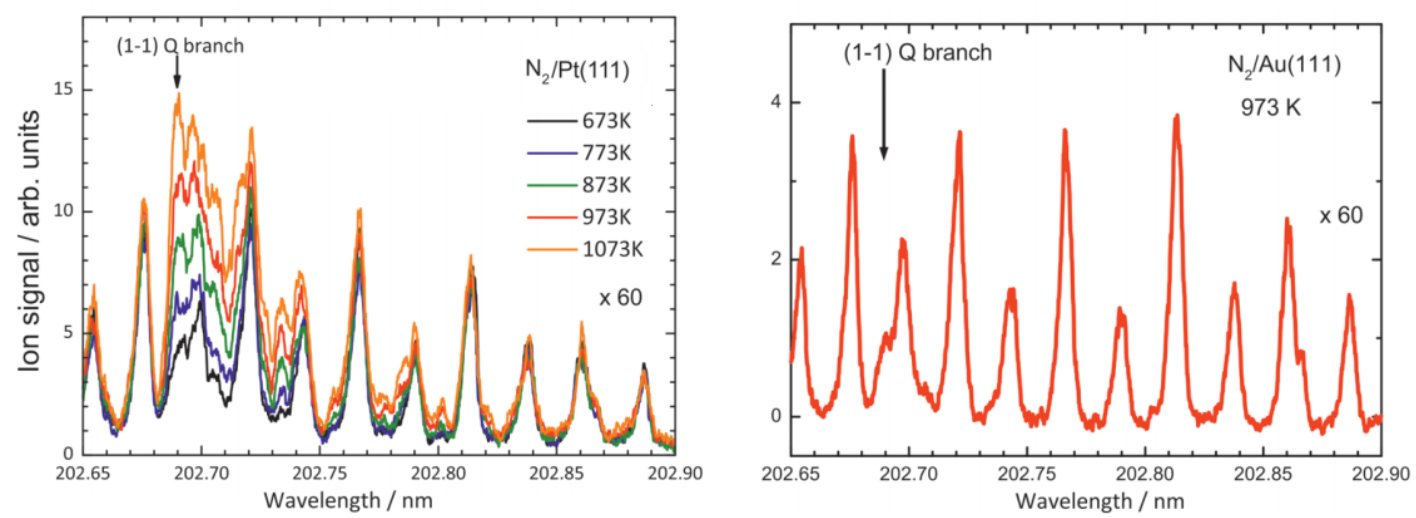

Figure 2.7: Vibrational excitation of $N_{2}$ : REMPI Spectra of scattered $N_{2}$. Left: Part of the (0-0) O branch and the (1-1) Q branch region of a beam of $E_{i}=0.7 \mathrm{eV}$ at different temperatures. Right: The much weaker excitation of $N_{2}$ scattered from Au(111) at $T_{s}=973 \mathrm{~K}$. Taken from Ref [39] .

While a signal of vibrationally excited $\mathrm{N}_{2}(v=1)$ could be detected with a $T_{\mathrm{s}}$ dependence after scattering from Pt(111), only a small signal (see arrow in Figure 2.7) was observed after scattering from $\mathrm{Au}(111)$ (see Figure 2.7). An upper limit of $P_{\mathrm{N}_{2}(v=0 \rightarrow 1)}=10^{-4}$ was estimated for $\mathrm{Au}(111)$, one magnitude lower than for Pt(111). The different vibrational excitation probabilities were explained by the differences in the electronic structure of Au and Pt. The $d$-orbitals of Au are lying $2 \mathrm{eV}$ below the Fermi level whereas 
the $d$-band of $\mathrm{Pt}$ is at the Fermi level. Three possible influences on the nonadiabaticity were discussed: (i) Due to the increased density-of-states at the Fermi level for Pt, more electron-hole pairs are available which can compensate the work function difference of $0.5 \mathrm{eV}$ between $\mathrm{Au}$ and Pt. (ii) With $d$-bands closer to the Fermi level, the chemisorption strength is increased and $\mathrm{N}_{2}$ can approach closer to the surface thereby increasing nonadiabatic coupling. (iii) Overlap between the orbitals of molecule and the surface leads to lowering of the bonding level energy in addition to the image charge stabilization, making charge-transfer more favorable.

Drawing a conclusion from experimental results of $\mathrm{NO}$ and $\mathrm{N}_{2}$ excitation measured from different metals, we state that (a) the work function or (b) the density-of-states at the Fermi level are possible properties of the surface that influence the nonadiabatic coupling strength.
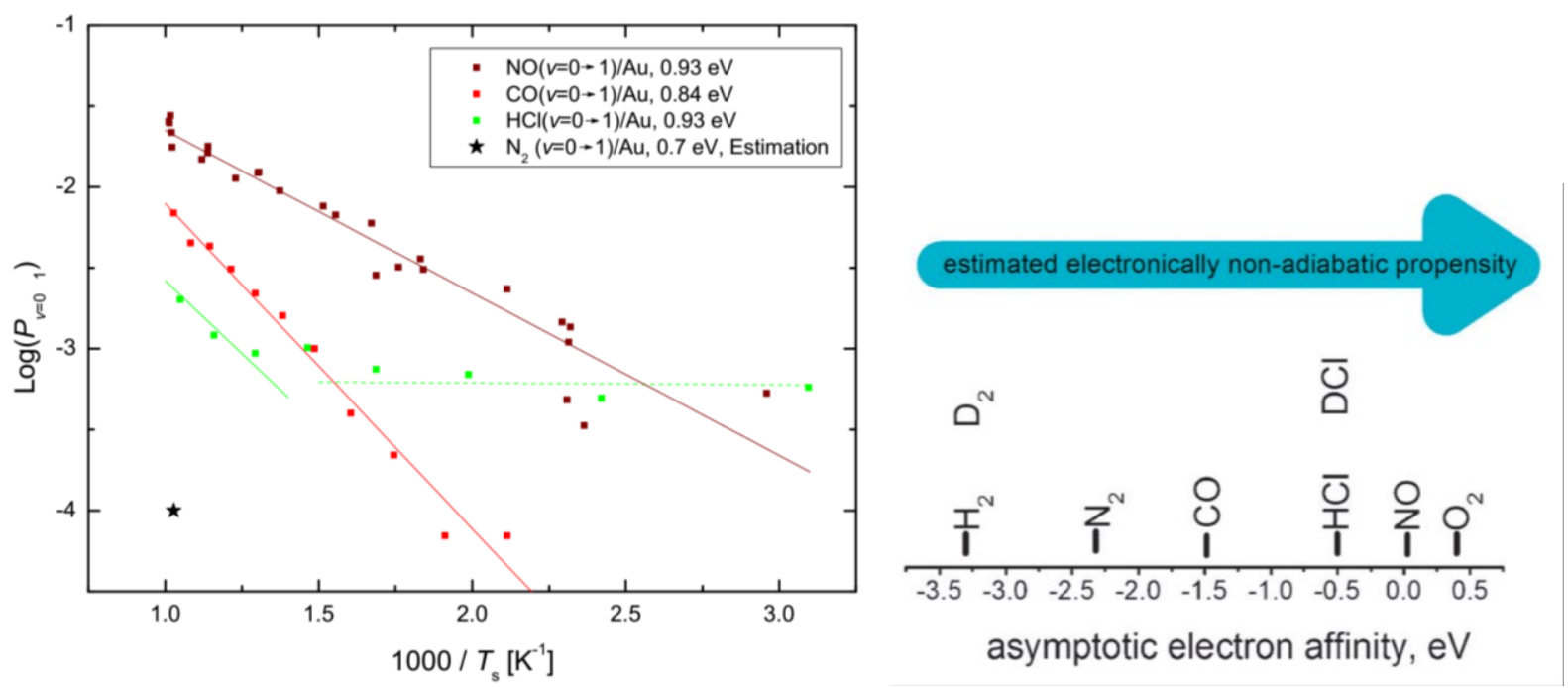

Figure 2.8: Nonadiabatic coupling strength for different molecules. Left: Arrhenius plots for vibrational excitation probabilities on $\mathrm{Au}(111)$ for $\mathrm{NO}$ [35], $\mathrm{CO}$ [40], $\mathrm{HCl}$ [41] and $\mathrm{N}_{2}$ [39]. The solid lines mark the nonadiabatic trend, for $\mathrm{HCl}$ a dashed line is drawn where excitation is dominated by an adiabatic mechanism. Right: Series of diatomics ordered according to their asymptotic electron affinity. Taken from Ref. [18].

Another way to compare the nonadiabaticity of different systems is to compare the excitation probabilities of different molecules for the same surface. Systematic experimental data is available for the excitation of the first vibrational state of the molecules $\mathrm{NO}[28,35], \mathrm{HCl}[33,41], \mathrm{CO}[29,40], \mathrm{N}_{2}[39]$ after scattering from $\mathrm{Au}(111)$ which is depicted for comparable incidence energies in Figure 2.8 (left). Instead of the excitation probability, pre-exponential factor $A$ is a better measure for comparison which takes into account the different vibrational energies. For the $\mathrm{HCl} / \mathrm{Au}$, both adiabatic and nonadiabatic contributions influence vibrational excitation and yield separated pre-exponential factors. Focusing on the nonadiabatic factors, we obtain the following trend: $A(\mathrm{NO} / \mathrm{Au})>A(\mathrm{CO} / \mathrm{Au}) \approx A(\mathrm{HCl} / \mathrm{Au})>>A\left(\mathrm{~N}_{2} / \mathrm{Au}\right)$. This can be understood in terms of the molecular electron affinity which was suggested as a further "intuitive proxy" to compare nonadiabaticity of different molecules [18]. The lower the electron affinity, the higher is the tendency to accept an electrons which can lead to nonadiabatic vibrational excitation. The electron affinities for a few diatomic molecules are shown in the diagram of Figure 2.8 (right) and are qualitatively in agreement with the trend of the pre-exponential factors.

For the investigated molecules, NO shows the highest tendency to undergo nonadiabatic effects that even excitation to higher vibrational states $\mathrm{NO}(v=0 \rightarrow 1,2,3)$ is reported [35]. It is however not clear, why $\mathrm{HCl}$ and $\mathrm{CO}$ exhibit nonadiabatic coupling of a similar order of magnitude although their affinities differ by about $1 \mathrm{eV}$. Apart from the adiabatic contribution, a further unique characteristic was found for the $\mathrm{HCl} / \mathrm{Au}$ system: Vibrational excitation probability is significantly increased for a vibrationally 
excited species. The excitation channel $\mathrm{HCl}(v=1 \rightarrow 2)$ exhibits enhanced excitation by a factor of 20 in comparison with the $\mathrm{HCl}(v=0 \rightarrow 1)$ channel [41] whereas only moderate enhancement was found for $\mathrm{NO}(v=2 \rightarrow 3)$ in comparison with $\mathrm{NO}(v=0 \rightarrow 1)$. The observation was discussed in context of the difference in dissociation barriers of both molecules on $\mathrm{Au}(111)$. While the calculated barrier of $\mathrm{NO}$ is $>3 \mathrm{eV}, \mathrm{HCl}$ dissociation will occur for lower energies. Additionally, investigations on the dissociation of $\mathrm{HCl}$ on $\mathrm{Au}(111)$ suggested that the dissociation barrier for vibrationally excited molecules was significantly decreased [42]. In an analogous manner it was argued that vibrational excitation can be increased for a system close to the dissociative transition state.

With regard to influence of the molecule on vibrational excitation, it can be concluded that the electron affinity determines the tendency for nonadiabatic interactions. Additional enhancement is possible for molecules in excited vibrational states, especially when they are energetically close to a transition state.

\subsubsection{Molecular Vibrational Relaxation}

After initial experiments on vibrational excitation it was suggested to learn about nonadiabatic effects by investigating the opposite effect of vibrational deexcitation. This approach yielded probably the most convincing experimental proof for the important role of surface electrons for vibrational energy transfer. Molecules are prepared in an excited vibrational state and scattering from a surface. The final vibrational distribution is obtained in a state-selective manner with the help of laser techniques. Comparison between a metal and an insulator surface as scattering target shows strong differences in the final state distribution. Figure 2.9 shows the vibrational distribution of highly excited NO after scattering from (a) $\mathrm{Au}(111)$ and (b) LiF [43].

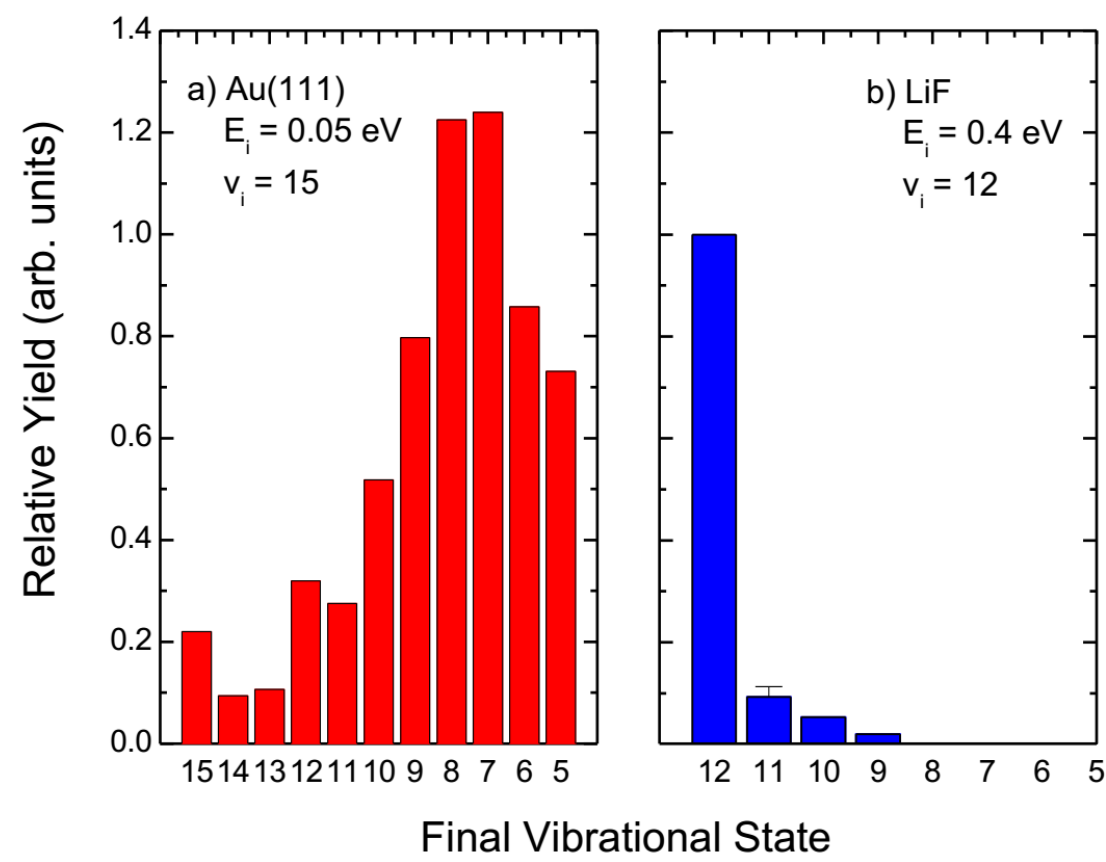

Figure 2.9: Vibrational state distribution after scattering of vibrationally excited NO from (A) Au(111) and (B) LiF. Scattering from a metal surfaces produces a broad vibrational distribution with molecules undergoing multiquantum relaxation. In contrast, after scattering from an insulator surface, most molecules keep the initial vibrational state. Taken from Ref.[43] .

After scattering from a metal, a broad final distribution is observed with a large amount of molecules having lost several quanta of vibrational energy. A significantly different distribution is found after scattering from an insulating surface. Most of the molecules remain in their initial vibrational state without undergoing relaxation. The obvious reason for the contrasting behavior is the difference in 
electronic structure of both surfaces. While NO can only interact with phonons on LiF due to the absence of metallic states, $\mathrm{Au}(111)$ provides a continuum of electronic states while can serve as a sink for molecular vibrational energy. This hypothesis was further corroborated by scattering experiments on low work function surfaces of $\mathrm{Cs}$-covered $\mathrm{Au}(111)$. Instead of measuring the final vibrational distribution, electron emitted from the surface were detected. The dependence of the electron emission on the initial vibrational state is depicted in Figure 2.10.

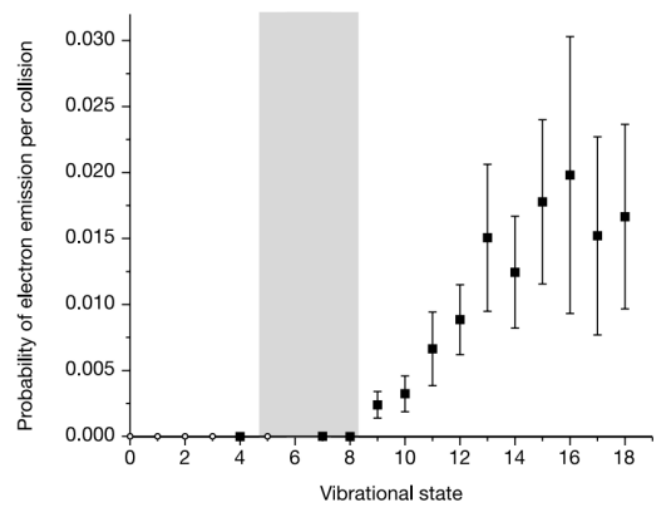

Figure 2.10: Electron emission probability after scattering of vibrational excited NO from a low work function surface. For low vibrational states, no electrons are detected. The onset of electron emission starts when the vibrational energy is slightly above the estimated work function ( $\Phi=1.3-1.6 \mathrm{eV})$. Taken from Ref. [44].

While the scattering of NO in low vibrational states $(v=0-8)$ did not result in electron emission, a clear threshold-like behavior was observed slightly above the work function of the material. This experiment suggests that vibrational energy of NO is directly converted into kinetic energy of electrons, providing additional evidence for nonadiabatic energy transfer events in molecule-surface scattering processes.

In regard to vibrational relaxation, systematic scattering data is only available for NO and CO. Especially scattering of vibrationally excited NO from $\mathrm{Au}(111)$ has been investigated for a number of incidence energies and vibrational states $(v=2,3,12,15)$ for a set of benchmark data to test theoretical models for nonadiabatic vibrational energy transfer.
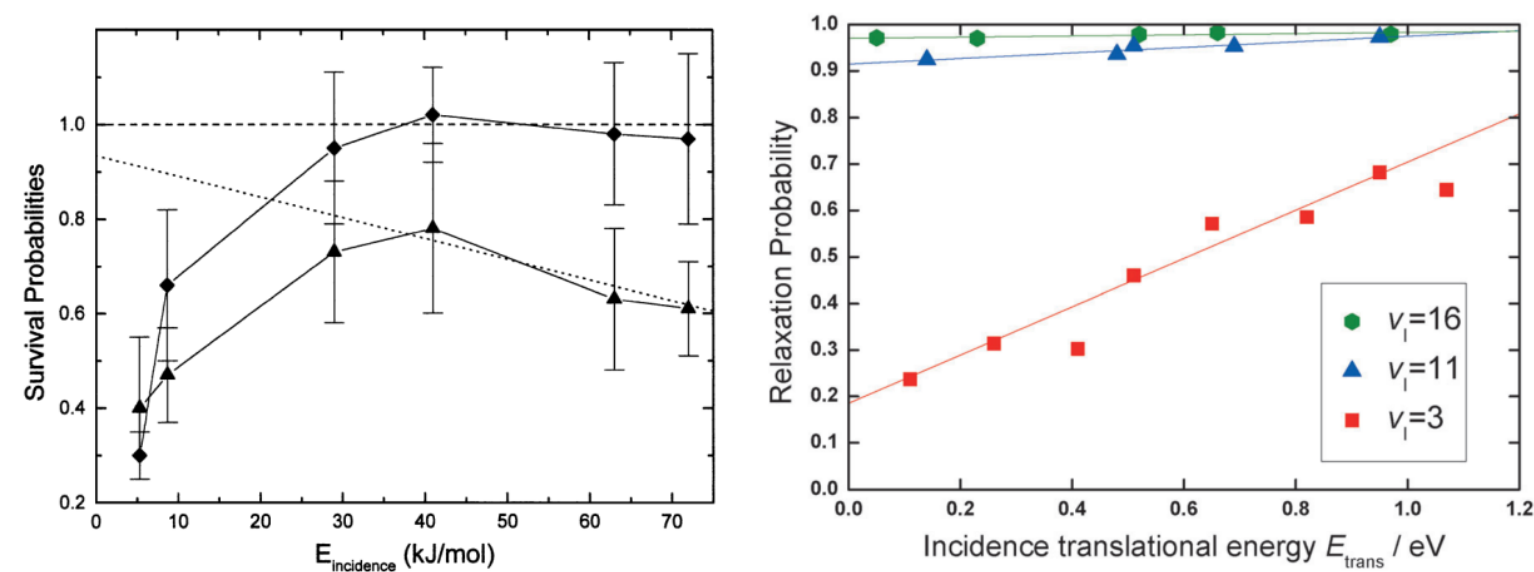

Figure 2.11: Vibrational relaxation of NO at different translational energies. Left panel: Survival probability of $N O(v=2)$ for several incidence energies (triangles). To distinguish the effect of trapping desorption, a survival probability of $N O(v=0)$ was detected separately (spades). Take from Ref. [45]. Right Panel: Relaxation probability for several incidence energies for NO( $v=3,11,16)$. Taken from Ref.[46].

An overview of these results is depicted in Figure 2.11. The left panel shows the amount of $\mathrm{NO}(v=2)$ that survives a collision at $\mathrm{Au}(111)$ [45] which is the survival probability $P_{\text {surv, }}$ whereas the counterpart, the relaxation probability $P_{\text {Relax }}=\left(1-P_{\text {surv }}\right)$ is depicted in the right panel for $\mathrm{NO}(v=3,12,15)$ [46]. 
Similarly as for excitation, we see an increase of relaxation for higher incidence energies. However, the scattering of highly vibrational states $(v=11,15)$ leads to such a substantial increase in relaxation probability that the effect of incidence energy is negligible. The same study reported an orientation effect. Motivated by theoretical predictions of a higher relaxation probability for NO with $\mathrm{N}$ oriented towards the $\mathrm{Au}(111)$ surface [47], it could experimentally be confirmed that this is true for vibrationally excited molecules $\mathrm{NO}(v=3,11)$. For the highest investigated vibrational state $\mathrm{NO}(v=16)$, the orientation effect becomes less crucial.

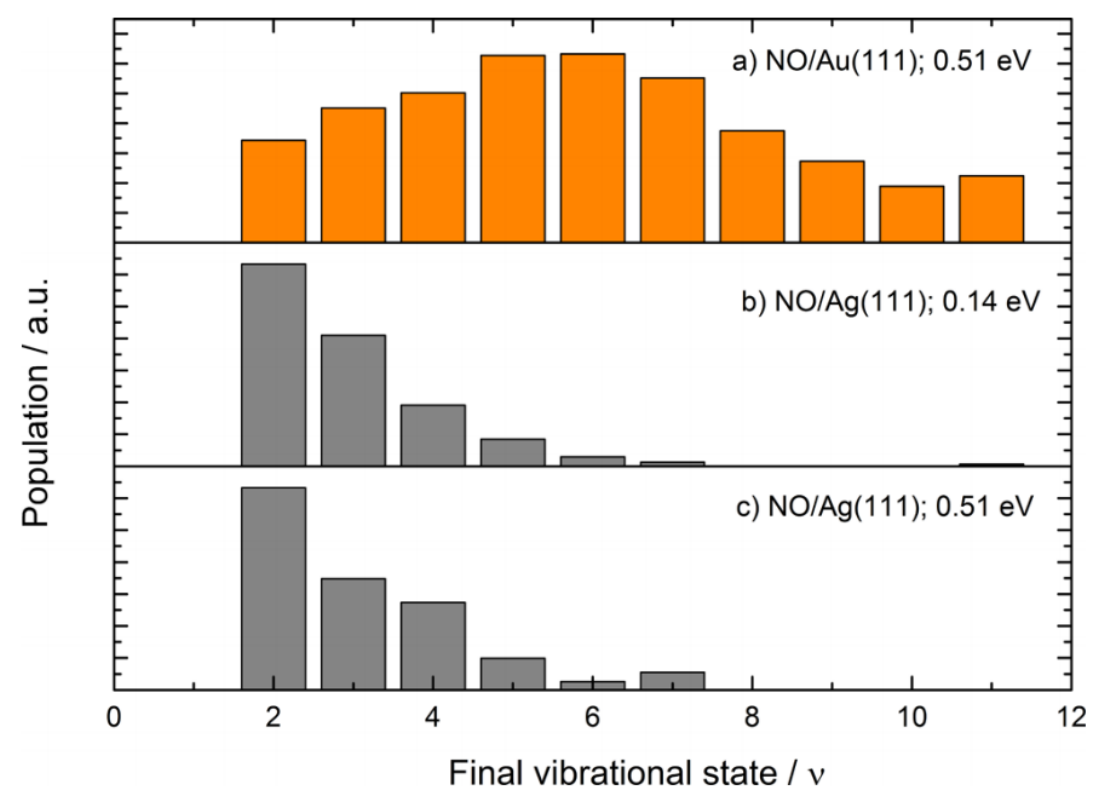

Figure 2.12: Vibrational relaxation of $N O(v=11)$ on $A u(111)$ and $A g(111)$. Taken from Ref. [48] .

A comparison between $\mathrm{NO}(v=11)$ scattering from $\mathrm{Au}(111)$ and $\mathrm{Ag}(111)$ showed that the surface has significant influence on the relaxation probability [48]. As can be seen in Figure 2.12, vibrational relaxation was strongly increased on the $\mathrm{Ag}(111)$ surface. On $\mathrm{Au}(111) \mathrm{NO}(v=11)$ loses an average of 5.1 vibrational quanta, in contrast, on $\mathrm{Ag}(111)$ the average loss is 8.1 vibrational quanta. In contrast to scattering from a $\mathrm{Au}(111)$ surface, the influence of incidence energy is only negligible on the $\mathrm{Ag}(111)$ surface. Two possible reasons were discussed to explain the increased relaxation. One the one hand, it was suggested that the lower surface work function ( $\mathrm{Au}: \phi=5.3 \mathrm{eV} ; \mathrm{Ag}: \phi=4.7 \mathrm{eV}$ ) could explain the observed behavior. One the other hand, because the energy loss of the $\mathrm{NO} / \mathrm{Ag}(111)$ system of similar magnitude as the system of NO scattered from $\mathrm{Cs}$ covered $\mathrm{Au}(111)$ which is a surface with significantly lower work function $(\phi=1.6 \mathrm{eV})$, it was postulated that the dissociation barrier of NO might influence the relaxation probability. DFT calculations predict a lower NO dissociation barrier for $\mathrm{Ag}(111)$ (3.1 eV) in comparison to $\mathrm{Au}(111)(3.6 \mathrm{eV})$ and with a highly excited state $\mathrm{NO}\left(v=11, E_{\text {vib }}=2.5 \mathrm{eV}\right)$, nonadiabatic interactions might be increased close to a transition state. 


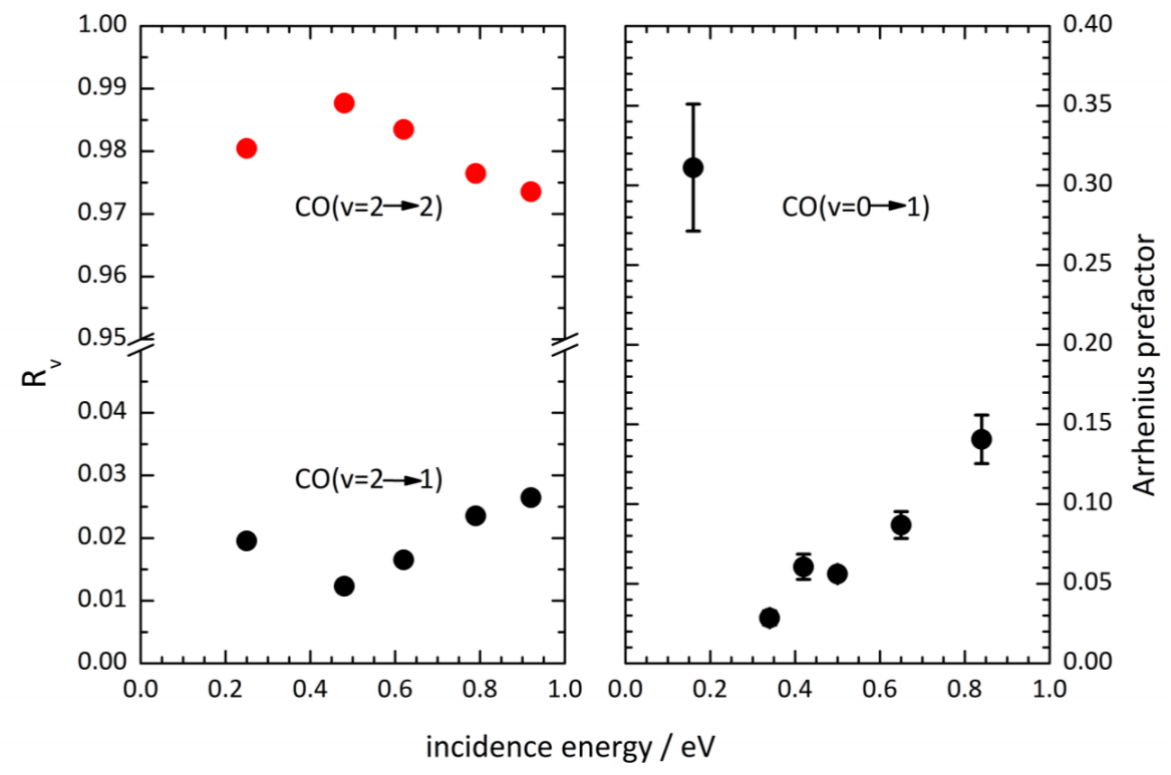

Figure 2.13: Nonadiabatic interactions of $\mathrm{CO}(v=2)$ scattering from $\mathrm{Au}(111)$ at different incidence energies. Left: Amount of $\mathrm{CO}(v=2)$ vibrational relaxation on $\mathrm{Au}(111)$ neglecting relaxation into $v=0$. Right: Arrhenius prefactor for CO for different incidence energies. Taken from Ref. [49].

Only low relaxation probabilities of $1-3 \%$ were observed for $\mathrm{CO}(v=2)$ scattering from a $\mathrm{Au}(111)$ surface. A small effect of the incidence energy could be identified which was similar to the effect obtained in vibrational excitation (see Figure 2.13). Nonadiabatic coupling is weaker than for the NO/Au(111) system which can be explained by the difference in electron affinity of both molecules (see Figure 2.8, right panel).

All in all, the amount of vibrational relaxation seems to be characterized by the same trends that have been observed for excitation. Higher Incidence energy leads to more relaxation, excluding cases in which strong relaxation is already present like high vibrational energy or a surface with strong nonadiabatic coupling. It is observed that surface characteristics can increase vibrational relaxation, suggested are the work function or the dissociation probability. Molecules with lower electron affinity exhibit less vibrational relaxation. 


\subsection{Properties of Thin Metal Films}

Although the term "thin film" is often found on books ("Handbook of Thin Films") or journals ("Thin Solid Films"), it is used to describe a vast field of different materials. Depending on the part of the scientific community, it defines film thicknesses stretching from sub-monolayer ranges $\left(10^{-10} \mathrm{~m}\right)$ to micrometers $\left(\sim 10^{-6} \mathrm{~m}\right)$. Even for a defined thickness range, the properties of the interfaces are strongly influenced by deposition method, growth conditions and of course the employed materials. Many films exhibit effects only for certain thicknesses due to a lot of possible reasons (for example quantum-size effects, interface effects or structural effects). Due to this variety, it impossible to cover this topic even in an introductory manner. This section will therefore be strongly restricted to the employed thin film system of $\mathrm{Ag} / \mathrm{Au}(111)$ and similar systems with a thickness of max. $50 \mathrm{ML}(\sim 25 \mathrm{~nm})$, consisting of two metals, grown in an epitaxial manner under UHV conditions. The focus will be on three aspects: The growth mode of thin films, thickness-dependent properties and the special group of bimetallic single layer systems. The last aspect is included because there might be a connection between the bonding character of a heteronuclear metal-metal bond and the scattering behavior in the presented work. However, this topic can only be treated in a general manner since there is no experimental data available for the system of $\mathrm{Ag} / \mathrm{Au}(111)$ but for many other bimetallic transition metal systems.

\subsubsection{Epitaxial Growth Mode of Thin Metallic Film Systems}

The $\mathrm{Ag} / \mathrm{Au}(111)$ films of the present work have been grown by molecular beam epitaxy. The term epitaxy describes a film growth that can be achieved with materials of similar lattice constants. Under UHV condition, the adsorbate material grows with the same lattice constant onto the crystalline substrate leading to clean, well-ordered monocrystalline surfaces. Surfaces of epitaxial films grown under UHV condition are therefore ideal for surface studies.

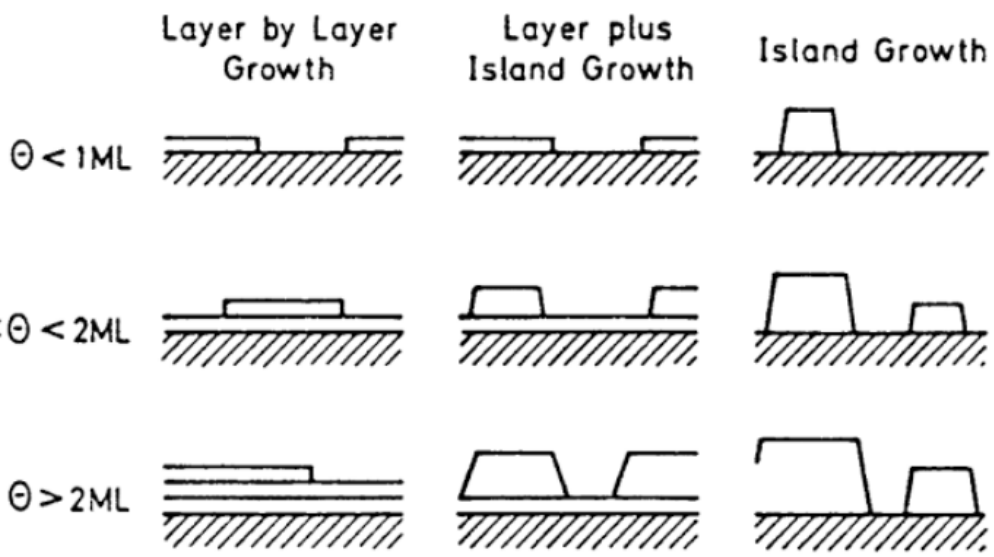

Figure 2.14: Schematic representation of the growth modes of a film for different coverage $(\theta)$ regimes. (a) Layer by-layer growth (Frank-van-der-Merwe, FM). (b) Layer-plus-island growth (Stranski-Krastanov, SK). (c) Island Growth (Vollmer-Weber, VW) (taken from Ref. [50]).

The growth mode of bimetallic layers systems is often described in a phenomenological manner. In general, three principal growth modes are distinguished (Figure 2.14): In the layer-by-layer growth (or Frank-van-der-Merwe, FM), the interaction between substrate and layer atoms is stronger than that in between neighboring layer atoms. A new layer starts to grow only if the previous one is completed. The opposite case with stronger interaction between the layer atoms than the overlayer-substrate interaction is the island growth (or Volmer-Weber growth, VW) leading to the growth of three- 
dimensional structures. An interesting case in between is the layer-plus-island growth (or StranskiKrastanov growth, SK). For this mode, the first or even several adsorbate monolayers are growing in a layer-by-layer fashion before island growth occurs. Different factors can account for this behavior, a certain lattice mismatch is present between substrate and adsorbate in many cases of that growth mode.

A simple approach to explain the growth mode for a certain metal-metal system is possible in terms of the surface or interface energy $\gamma$ of the involved materials. This measure denotes the characteristic free energy (per unit area) to create an additional piece of surface or interface. If the substrate has a surface energy $\gamma_{S}$, the adsorbate $\gamma_{A}$ and the interface between the two $\gamma_{I}$, the condition $\gamma_{S}>\gamma_{A}+\gamma_{I}$ will favor the covering over the substrate by spreading of the adsorbate, while $\gamma_{S}<\gamma_{A}+\gamma_{i}$ will favor non-wetting. Although this criterion could be supported by experiments, the prediction of a growth mode is still a complex problem. Approximate values for $\gamma_{A}$ and $\gamma_{S}$ may be known but rarely for $\gamma_{I}$. In the case of $\mathrm{Ag} / \mathrm{Au}(111)$, surfaces energies are reported as $\gamma_{\mathrm{Au}}=2.66 \mathrm{~J} \mathrm{~m}^{-2}$ and $\gamma_{\mathrm{Ag}}=1.20 \mathrm{~J} \mathrm{~m}^{-2}$, predicting a layer-by-layer growth [12]. Table 1 contains an overview of some layer systems and their growth modes showing that many bimetallic layer system follow the same trend.

Table 1: Overview of some layer systems and their growth modes FM, SK, VW (taken from Ref. [50]).

\begin{tabular}{|c|c|c|}
\hline $\begin{array}{c}\text { Layer Growth } \\
\text { Frank-van-der-Merwe (FM) }\end{array}$ & $\begin{array}{l}\text { Layer plus island growth } \\
\text { Stranski-Krastanov (SK) }\end{array}$ & $\begin{array}{l}\text { Island growth } \\
\text { Volmer-Weber (VW) }\end{array}$ \\
\hline $\begin{array}{l}\text { many metals on metals } \\
\text { e.g., } \mathrm{Pd} / \mathrm{Au}, \mathrm{Au} / \mathrm{Pd}, \mathrm{Ag} / \mathrm{Au} \text {, } \\
\mathrm{Au} / \mathrm{Ag}, \mathrm{Pd} / \mathrm{Ag}, \mathrm{Pb} / \mathrm{Ag} \text {, } \\
\mathrm{Pt} / \mathrm{Au}, \mathrm{Pt} / \mathrm{Ag}, \mathrm{Pt} / \mathrm{Cu}\end{array}$ & $\begin{array}{l}\text { some metals on metals, } \\
\text { e.g., } \mathrm{Pb} / \mathrm{W}, \mathrm{Au} / \mathrm{Mo} \text {, } \\
\mathrm{Ag} / \mathrm{W}, \\
\text { rare gases on graphite }\end{array}$ & $\begin{array}{l}\text { most metals on alkali } \\
\text { halides } \\
\text { most metals on } \mathrm{MgO} \text {, } \\
\mathrm{MoS}_{2} \text { graphite, } \\
\text { mica }\end{array}$ \\
\hline $\begin{array}{l}\text { alkali halides on alkali } \\
\text { halides } \\
\text { III-V alloys on III-V alloys } \\
\text { e.g., GaAlAs/GaAs, } \\
\text { InAs/GaSb } \\
\text { GaP/GaAsP, InGaAs/GaAs }\end{array}$ & $\begin{array}{l}\text { many metals on semi- } \\
\text { conductors, e.g.: } \\
\mathrm{Ag} / \mathrm{Si}, \mathrm{Ag} / \mathrm{Ge}, \\
\mathrm{Au} / \mathrm{Si}, \mathrm{Au} / \mathrm{Ge}, \\
\mathrm{Al} / \mathrm{GaAs}, \mathrm{Fe} / \mathrm{GaAs}, \\
\mathrm{Sn} / \mathrm{GaAs} \\
\mathrm{Au} / \mathrm{GaAs}, \mathrm{Ag} / \mathrm{GaAs}\end{array}$ & \\
\hline $\begin{array}{l}\text { IV semiconductors on some } \\
\text { III-V compounds, e.g. } \\
\text { Ge/GaAs, Si/GaP, } \alpha \text {-Sn/InSb } \\
\text { transition metals on } \mathrm{Si} \\
\mathrm{Pt} / \mathrm{Si}, \mathrm{Pd} / \mathrm{Si}, \mathrm{Ni} / \mathrm{Si} \text { (silicides) }\end{array}$ & & \\
\hline
\end{tabular}



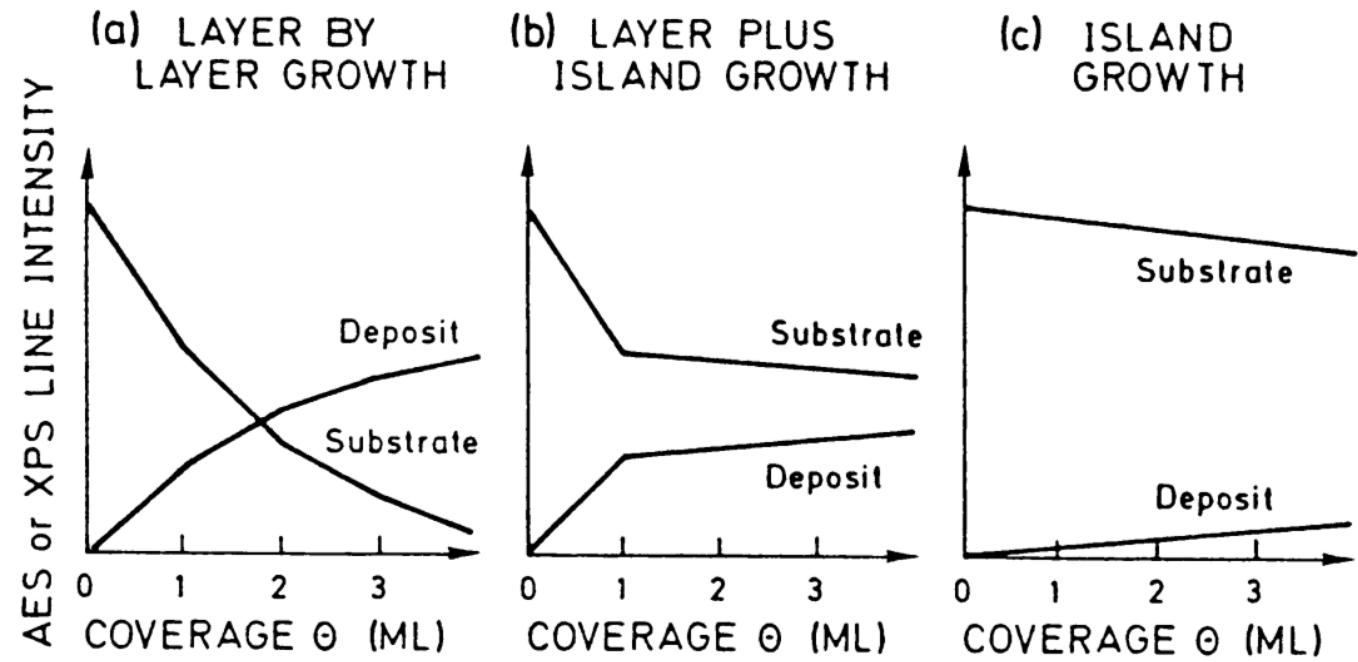

Figure 2.15: Schematic AES line intensities from deposit an substrate versus amount of deposited material (coverage $\Theta$ ) for (a) layer-by-layer growth (FM), (b) layer-plus-layer (SK), (c) island growth (VW) (taken from [50]).

One classic standard method to distinguish the different growth mechanisms is the use of Auger electron spectroscopy (AES) [9]. AES signal intensities of substrate and adsorbate as a function of coverage show characteristic patterns as shown in Figure 2.15. In the layer-by-layer mode, the AES peak intensity of both deposit and substrate shows distinct breaks, with the first break being an indication of the completion of the first monolayer [51]. In contrast, such breaks are absent in the island growth. Due to the growth of three-dimensional structures, large areas of the substrate remain free of deposit and the substrate signals are less suppressed than in the layer-by-layer mode. In the layer-plus-island growth, a single break after the first completed monolayer is found before the trend continues in a manner observed for the island growth mode.

Because of the similar lattice constants, $\mathrm{Ag}(4.079 \AA)$ ) and $\mathrm{Au}(4.065 \AA)$ [52], thin films of $\mathrm{Ag}$ and $\mathrm{Au}$ belonged to the first investigations on bimetallic systems [53] and early AES measurements characterized the room temperature growth mode as a layer-by-layer type [54]. Those findings were confirmed by transmission electron microscopy (TEM) and scanning tunneling microscopy (STM) [12,55-57].

\subsubsection{Electronic Surface Properties of Thin Metallic Films}

Adsorbed metal atoms on a substrate have a strong impact on the electronic properties of a surface. In this short overview, it will be focused on the electronic states that are close to the Fermi level because it is assumed that those might have the biggest influence. Observations that reflect the electronic effects of metal overlayers are (1) a change of the work function and (2) a shift of electronic surface states with increasing film thickness.

The work function $\phi$ of a material is often defined as the difference of two energetic levels, the vacuum level $E_{\mathrm{vac}}$ and the Fermi level $E_{\mathrm{F}}$ :

$$
\phi=E_{\mathrm{vac}}-E_{\mathrm{F}}
$$

It can be regarded as a barrier that has to be overcome to leave the bulk crystal. However, this is a very simplified approach ignoring surface characteristics. It cannot explain any changes of the surfaces, for example the difference between work functions of surfaces of the same material but a different miller index. 
Additional contributions have to be taken into account, one of those is the effect of surface dipole. In the simple quantum mechanical model of a particle-in-a-box with finite barriers, the electronic wave function is not zero at the surface but rather "spills out" into the vacuum with an exponentially decay. Using that model to describe the surface, the bulk electrons have a non-zero probability outside that barrier. This gives rise to the dipole layer at the surface which the emitted electron must pass through. Similar effects occur when steps are present on the surface which also modify the work function. Two examples of this phenomenon, the Smoluchowski effect, are the auto-epitaxy of W/W(110) [58] and $\mathrm{Pt} / \mathrm{Pt}(111)[59]$.

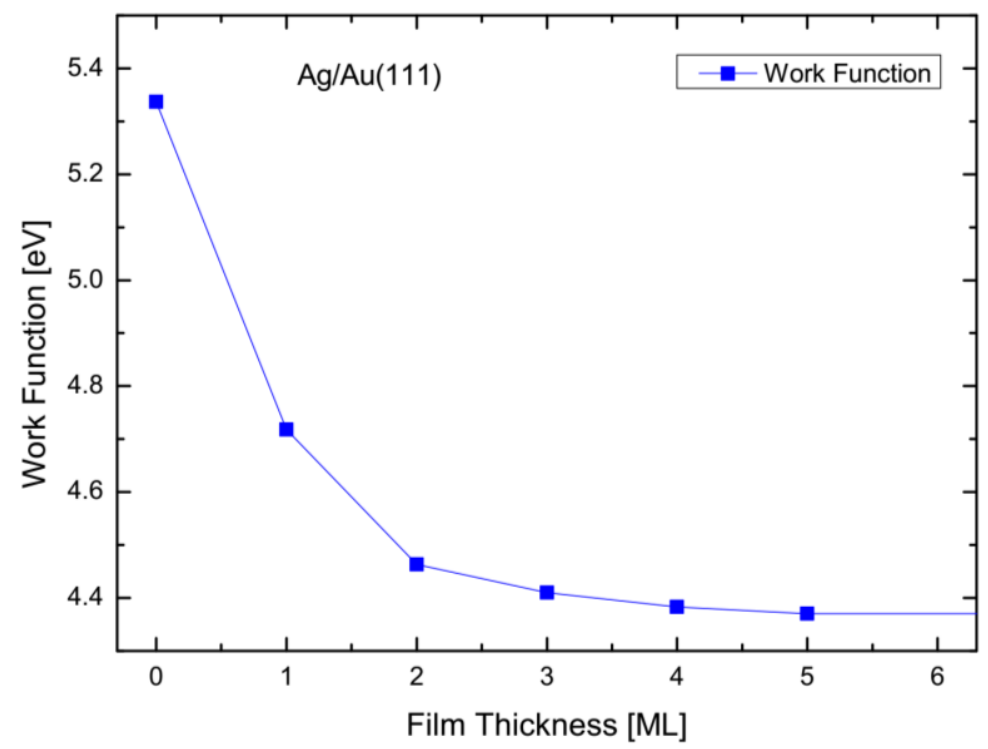

Figure 2.16: Thickness dependence of the work function of Ag/Au(111) (data from Ref. [12]).

In the case of $\mathrm{Ag}$ on $\mathrm{Au}(111)$, the adsorbate material has a lower work function, leading to a strong decrease within the first monolayers as shown in Figure 2.16. The linear behavior measured during the growth of a single monolayer ( 0 to $1 \mathrm{ML}, 1 \mathrm{ML}$ to $2 \mathrm{ML}, \ldots$ ) leads to pronounced changes in the slope at 1 and $2 \mathrm{ML}$ and is in agreement with the layer-by-layer growth [11]. It is worth pointing out that the "breaks" at full monolayer coverage are similar to the ones observed in the Auger signals (see Figure 2.15, left panel).

As predicted by Shockley [60] and Tamm [61] and experimentally confirmed by photoelectron spectroscopy [62], electronic surface states exist close to the Fermi level of a material. At the surface, the periodic structure of a lattice is abruptly terminated, leaving atoms with unsatisfied valence. Any influence, e.g. adsorbates, strongly determine the characteristics of the surface states. Figure 2.17 (left) shows photoelectron spectra of $\mathrm{Ag} / \mathrm{Au}$ for different film thicknesses in the region of $\sim 1 \mathrm{eV}$ below the Fermi level. The peak position of a surface state is strongly dependent on film thickness up to reaching the bulk value at $7 \mathrm{ML}$ with the strongest quantitative changes in the first 3-4 ML. Moreover, it can be observed that between completed monolayers, we don't observe a shift of the surface state but a mix of two surface states. In Figure 2.17 (left) that is most pronounced in the spectrum of $3.4 \mathrm{ML}$ showing a strong peak at $190 \mathrm{meV}$ (from the areas covered of $3 \mathrm{ML} \mathrm{Ag} / \mathrm{Au}$ ) and a weaker peak closer to the Fermi level (from the areas covered with $4 \mathrm{ML} \mathrm{Ag/Au}$ ). 

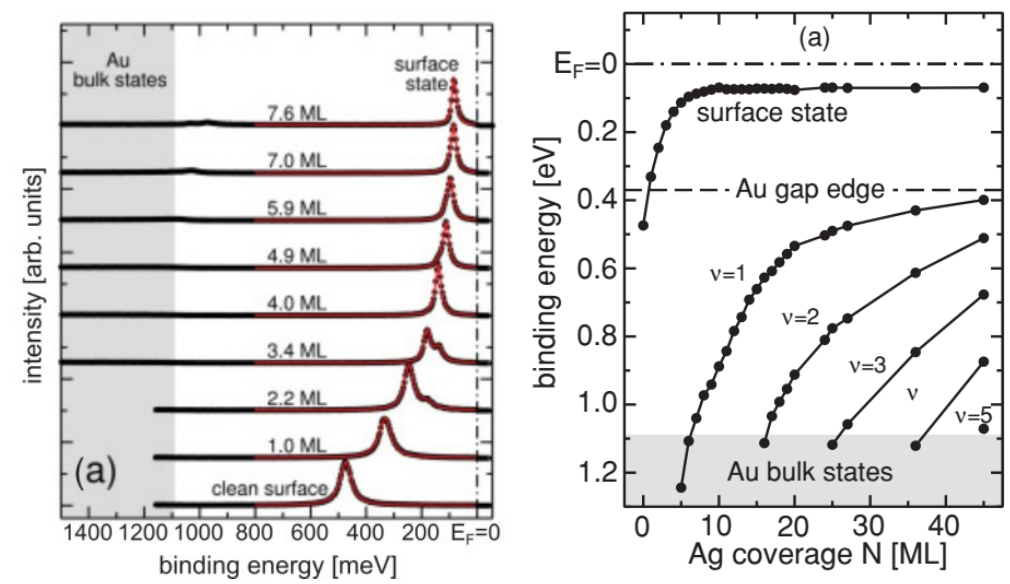

Figure 2.17: Left: Energy distribution curves at normal emission of Ag/Au(111) for different film thicknesses. Right: Binding energy of the surface state and the quantum well states as a function of film thickness (both taken from Ref. [10]).

If we want to distinguish between two different electronic effects due the change of film thickness, surface state energy and the work function, note that the representation in Figure 2.17 might be inconvenient. The spectrum is following the conventions of photoelectron spectroscopy, for which the Fermi level $E_{\mathrm{F}}$ is determined for every film thickness and set to the origin $\left(E_{\mathrm{F}}=0 \mathrm{eV}\right)$. A full picture about the surface shift can be obtained with $E_{\mathrm{vac}}=0 \mathrm{eV}$ as a common reference. Taking the change from $\mathrm{Au}(111)$ to $1 \mathrm{ML} \mathrm{Ag} / \mathrm{Au}$ as an example, the total shift of the surface state is $\sim 0.8 \mathrm{eV}$, obtained by adding the shift of the binding energy of Figure $2.17(\sim 0.2 \mathrm{eV})$ to the work function shift ( $\sim 0.6 \mathrm{eV})$ (see Figure 2.16).

Due to its importance in the context of thin films, the phenomenon of quantum size effects (QSE) in thin film systems should be mentioned. In a number of ultrathin film systems, the limitation in one dimension can lead to a confinement effect for the electrons within the overlayer. This situation can arise if the electronic states are localized at the surface and to not overlap with the electronic bands of the substrate. As a consequence, the energy of those quantum well states are strongly depending on the film thickness similarly as modeled in a particle-in-a-box picture with a finite potential wall.

Experimentally, quantum well states can be directly detected by photoelectron spectroscopy[63] and were reported to influence other properties of thin films, e.g. epitaxial growth [64], the work function $[65,66]$ and magnetic properties with the most prominent example of the giant magnetorestistance in certain multi-layered structures [67], awarded with the Nobel price in 2007. Moreover, there are studies of quantum size effects regarding the interaction with molecules, the reactivity of $\mathrm{O}_{2}$ with film of $\mathrm{Pb} / \mathrm{Si}[68]$ and $\mathrm{Mg} / \mathrm{Si}$ [69]. In the latter case, films with higher reactivity were also found to exhibit higher maximum signal in the measurement of chemicurrents, a nonadiabatic effect with the excitation of electron-hole pairs.

For $\mathrm{Ag} / \mathrm{Au}(111)$, quantum well states were observed with photoelectron spectroscopy to emerge in an energy window of $1 \mathrm{eV}$ below the Fermi level [70]. It was shown that the first states can be localized for thicknesses above $7 \mathrm{ML}$ [10]. Figure 2.17 (right panel) shows the binding energy as a function of film thickness between 0-45 ML with up to five quantum well states. 


\subsubsection{Single Layer Effects in Bimetallic Systems}

Among all thin film system, a special group consists of bimetallic systems with a single deposited monolayer. It was observed that those systems exhibited unique properties limited on the single bimetallic system but not present (or at least not as pronounced) for higher film thicknesses. Such simple adsorbate-substrate systems are interesting as model systems because synergistic effects can be studied. In the field of catalysis, two widely accepted mechanisms due to the effect of a second material are the ensemble effect and the ligand effect [71]. For a catalyst with the materials $A$ and $B$, the ensemble effect occur due to the influence of a changed number of binding sites, for example the selective blocking of sites at the surface of material $A$ by the addition of chemically inert $B$ atoms. The term ligand effect is used when electronic effects dominate, e.g. charge transfer or electronic perturbations induced by neighboring atoms of the other element.

One characteristic of bimetallic monolayer systems is the strong binding energy of the heteronuclear metal-metal bond. As an example, multiple overlayers of $\mathrm{Pd} / \mathrm{Re}$ exhibit a peak in temperature programmed desorption (TPD) experiments with a binding energy of $\sim 391 \mathrm{~kJ} / \mathrm{mol}$, similar to the sublimation energy of bulk $\mathrm{Cu}(\sim 378( \pm 20) \mathrm{kJ} / \mathrm{mol})[72]$. In contrast, for a peak that was assigned to a single overlayer, a binding energy of $\sim 416 \mathrm{~kJ} / \mathrm{mol}$ was determined. An overlayer of $\mathrm{Cu} / \mathrm{Re}$ showed similar effects. Further experiments showed that the heteronuclear bonding has a strong impact on chemical interactions. TPD measurements which examine the binding energy of $\mathrm{CO}$ became a standard technique to compare different bimetallic systems showing that the $\mathrm{CO}$ desorption temperature of a metal overlayer is strongly influenced by the choice of the substrate. Investigations of the core levels by $\mathrm{x}$-ray photoelectron spectroscopy (XPS) found that peaks of a single metal overlayer are shifted in comparison with the bulk material. This was interpreted as an effect of electronic perturbation induced by the metallic bonding. Figure 2.18 shows the strong correlation between the XPS core level shift and the CO TPD maximum.

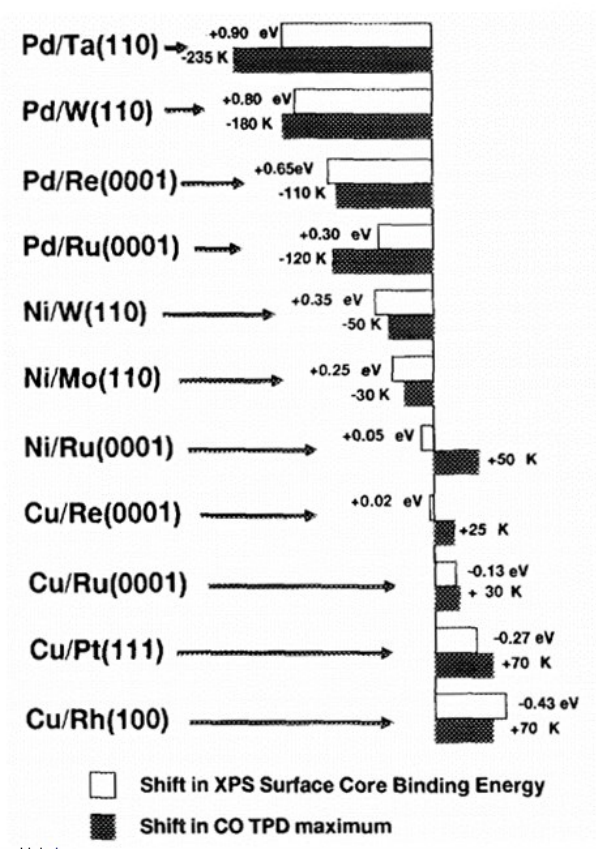

Figure 2.18: Correlation between the shifts in surface core-level binding energy and the shift in CO TPD maximum (taken from Ref. [73]).

No experimental data is available about single monolayer effects of the $\mathrm{Ag} / \mathrm{Au}(111)$ system. Estimations might be possible by comparison with a chemically similar system or from theoretical 
calculations. Core level shifts have been observed for $\mathrm{Cu} / \mathrm{Au}$, showing that even systems of materials with filled $d$-orbitals can have those properties [74]. A second indication is the comparison of the surface energies of the different metals. The stability of the metal-metal bond increases if the overlayer has a lower surface energy than the substrate which is the case for $\mathrm{Ag} / \mathrm{Au}$ [12]. A semi-empirical model based the surface energies predicts that the adsorption energy of the $\mathrm{Ag} / \mathrm{Au}$ layer is increased by $\sim 30 \mathrm{~kJ} / \mathrm{mol}$ in comparison to an $\mathrm{Ag} / \mathrm{Ag}$ layer system [75]. Furthermore, a theoretical model based on density functional theory (DFT) calculations was proposed that explains electronic effects of bimetallic systems by the shift of the $d$-band center in the metal overlayer [76]. The model was able to reproduce the trend of shifts in the $\mathrm{CO}$ adsorption energy for a number of systems and predicts a small shift of the $d$-band towards the Fermi level for the $\mathrm{Ag} / \mathrm{Au}$ system. Those predictions have to be treated with care, it is not clear if those materials with filled $d$-orbitals correctly described. In an earlier publication of the same group, DFT calculations failed to provide good results of $\mathrm{CO}$ chemisorption energies for noble metals surfaces [77]. Nevertheless, it seems that there is no bimetallic layer system that does not show extraordinary effects for a single layer system, therefore it cannot be ruled out that those effects are present for the $\mathrm{Ag} / \mathrm{Au}(111)$ system as well. 


\section{Experimental Details and Methods}

The experimental section consists of two parts. The first part focuses on a separate setup that was employed to grow and characterize ultrathin silver films, including a small UHV chamber, a sample transfer system and a home-built evaporator. After introduction of the employed devices, special emphasis will be on the film deposition process and the characterization of the produced silver films. After the completion of those initial tests, the film preparation stage was attached onto an already existing apparatus where molecular beam scattering experiments were performed. The second part of this chapter concentrates therefore on the molecular beam scattering apparatus, involved laser systems and experimental details for the translational/vibrational energy transfer investigations.

\subsection{Growth and Characterization of Ag/Au(111) Samples}

\subsubsection{UHV Chamber Setup}

\section{Test Chamber}

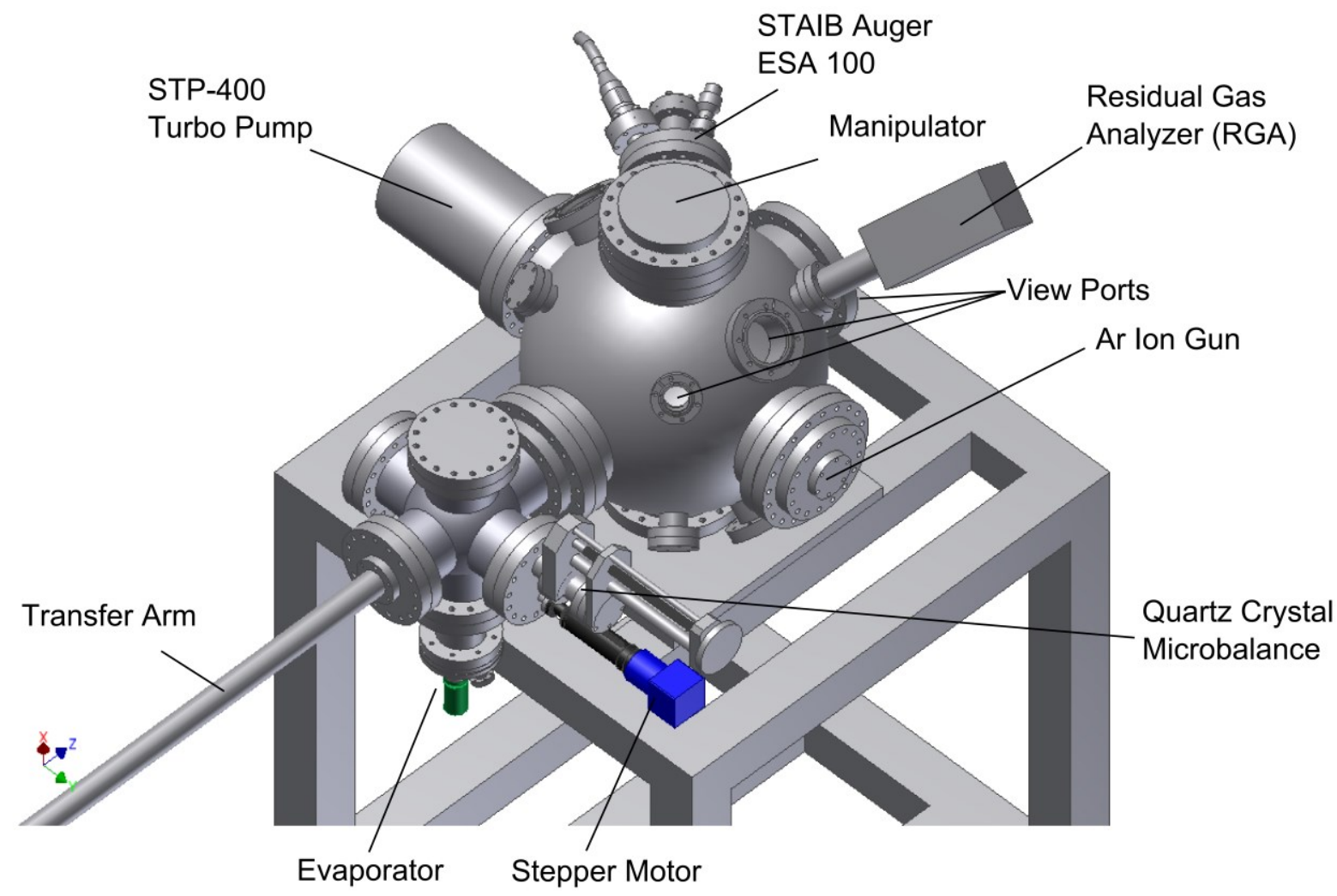

Figure 3.1: Sketch of the small UHV chamber for the film growth/characterization experiments.

Preparation and characterization of the thin films were performed in a spherical chamber (referred to as "test chamber") which is sketched in Figure 3.1. It consists of the main chamber (analysis stage) with a double cross (sample preparation stage) containing the evaporator. The transfer arm and a special design of the manipulator form the transfer system. UHV conditions are achieved with a turbo pump (Seiko Seiki STP 400, $400 \mathrm{~L} / \mathrm{s}$ ) which is backed by an additional booster pump (Pfeiffer HiPace 80, $67 \mathrm{~L} / \mathrm{s}$ ). The base pressure is $3.5 \times 10^{-10}$ Torr which rises to low $10^{-9} \mathrm{Torr}$ during film deposition. The residual gas 
analyzer (RGA) detected mainly $\mathrm{H}_{2}$ which desorbs from the glowing filament and other hot parts of the evaporator.

\section{Transfer System}

Adding additional functionality to a UHV chamber is generally a trivial task as long as free flanges are available. However, the use of an evaporator and producing a silver beam puts higher requirements on the experimental design: How can one prevent other sensitive analysis tools from getting coated and obtaining damage by the evaporated metal film? One solution to this problem is a spatial separation between film growth and sample characterization. In the present design, the evaporation process takes place in a separate part (double cross) so that all devices in the analysis stage remain unaffected. The transport of the substrate, a $\mathrm{Au}(111)$ crystal is possible via a sample transfer system.

The movable part containing the sample is referred to as the "shuttle" depicted at Figure 3.2 (left, center). On the bottom of the left panel, we see two dovetail-shaped "feet" of stainless steel which are mechanically fixed to a $3 \mathrm{~mm}$ sapphire plate. In a addition to electrical insulation, sapphire provides ideal properties regarding heat conduction because it exhibits good heat transport only at low temperatures. With those properties, it screens the manipulator when the sample is resistively heated but allows efficient sample cooling. On top of the sapphire plate, both feet are in electrical contact via two tantalum wires pressed into the notches of a $\mathrm{Au}(111)$ crystal (orientation accuracy better than $0.1^{\circ}$, purity $99.9999 \%$, MaTeck $\mathrm{GmbH}$ ). The sample temperature is determined by a thermocouple of type $K$, its measurement junction is fixed into a hole at the side of the crystal. The wires are connected with two contacts made of the corresponding material chromel/alumel at the bottom of the shuttle (Figure 3.2, central panel). When placing the shuttle into the reception, it is in the correct position to establish a connection between shuttle and reception. A hole (visible in Figure 3.2, right panel) contains a female thread which can be connected to the magnetic transfer arm. The sample connected to the transfer arm is depicted in Figure 3.3 (left panel).
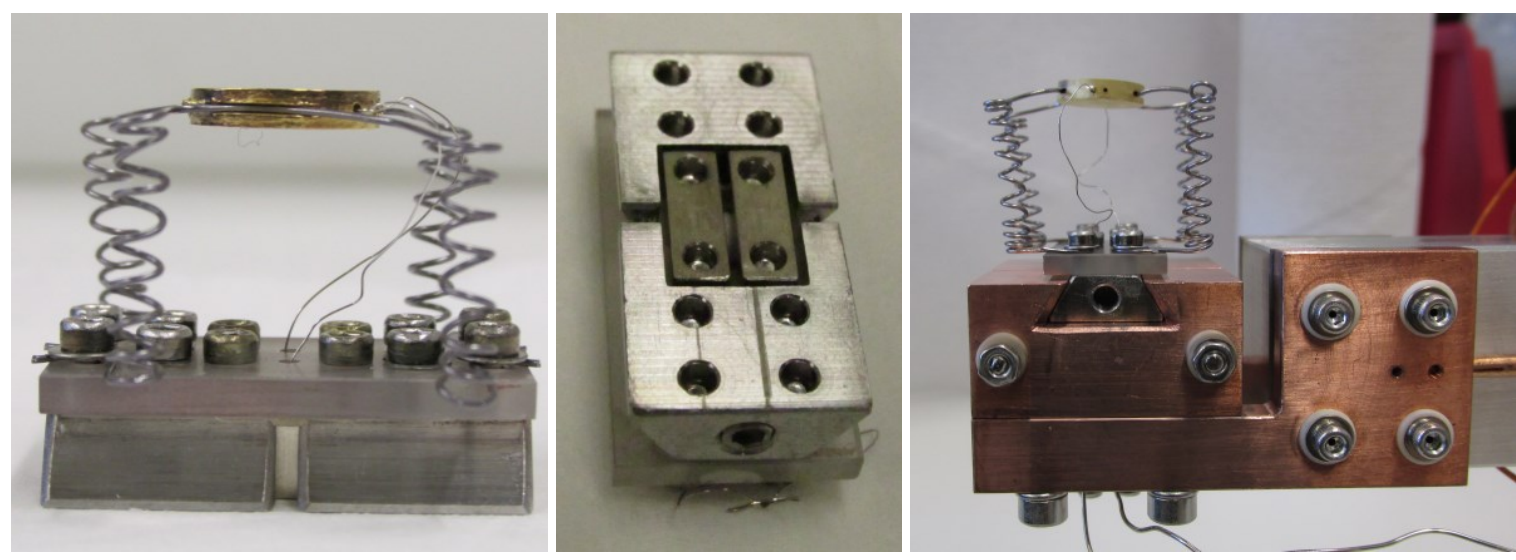

Figure 3.2: The mobile shuttle. Left panel: Side profile of the transfer sample with Au(111) crystal, tantalum wires, sapphire plate, feet (top to bottom). Central panel: Bottom view of the shuttle (without the Au(111) crystal) showing the chromel/alumel connections electrically isolated from each other and from the dovetail feet. Right panel: Different side profile showing shuttle connected to the reception. The thread used for the connection to the transfer arm is visible.

The outer tip of the transfer arm (Figure 3.3, central panel) conatins an additional feature: the hex key has the correct size to tighten the set screws at the back of the reception (Figure 3.3, right). That way, good electric contact between shuttle and reception is established and the sample temperature can be read out. The biggest advantage of this design is that it enables two contrary requirements of stability and flexibility: On the one hand, with tightened set screws, the shuttle is in a fixed position 
mantaining good electric contact. On the other hand, when loosened, a smooth transfer can be carried out avoiding stress and any damage to the system.

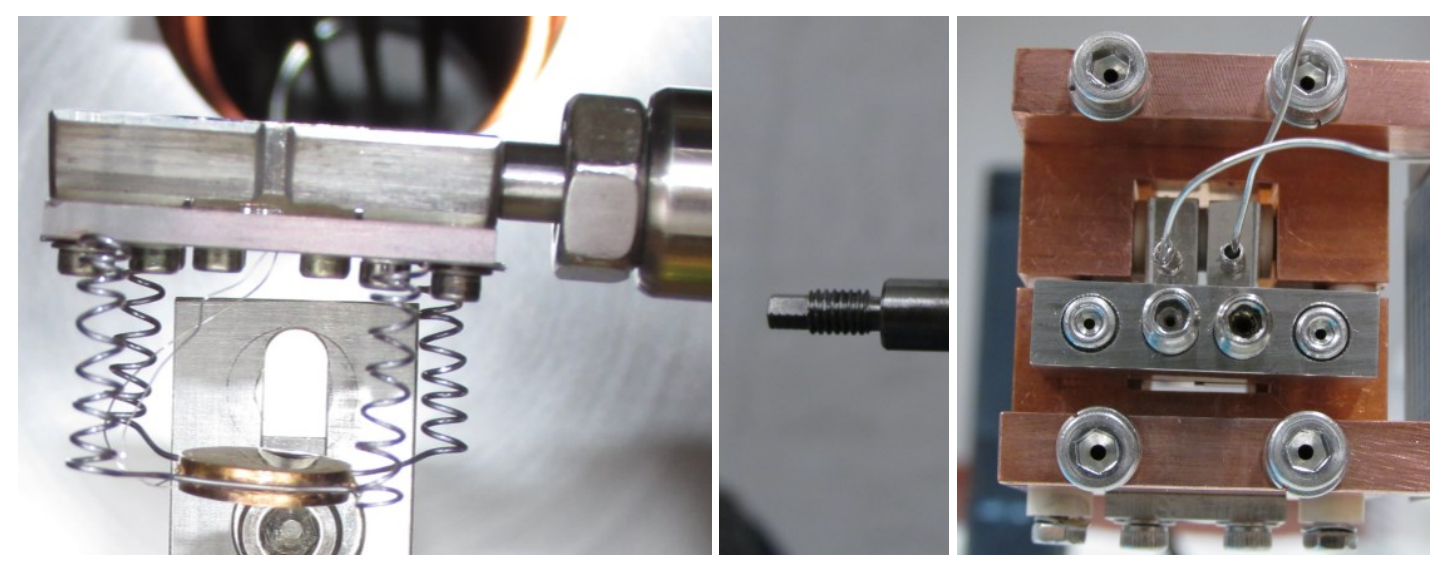

Figure 3.3: Double functionality of the transfer arm. Left panel: Transfer arm connected to the shuttle. Central panel: Outer tip of the transfer arm with the hex key to tighten the set screws at the manipulator and male thread to connect to the shuttle. Right panel: Set screws at the back of the manipulator. When tightened, the shuttle is mechanically fixed and temperature readout is possible.

\subsubsection{Film Deposition}

\section{Evaporator Design}

The evaporator used for this experiments is a home-built setup with the working principle of a thermal evaporator. Wiring diagram and a cross section of a 3D sketch are shown in Figure 3.4.
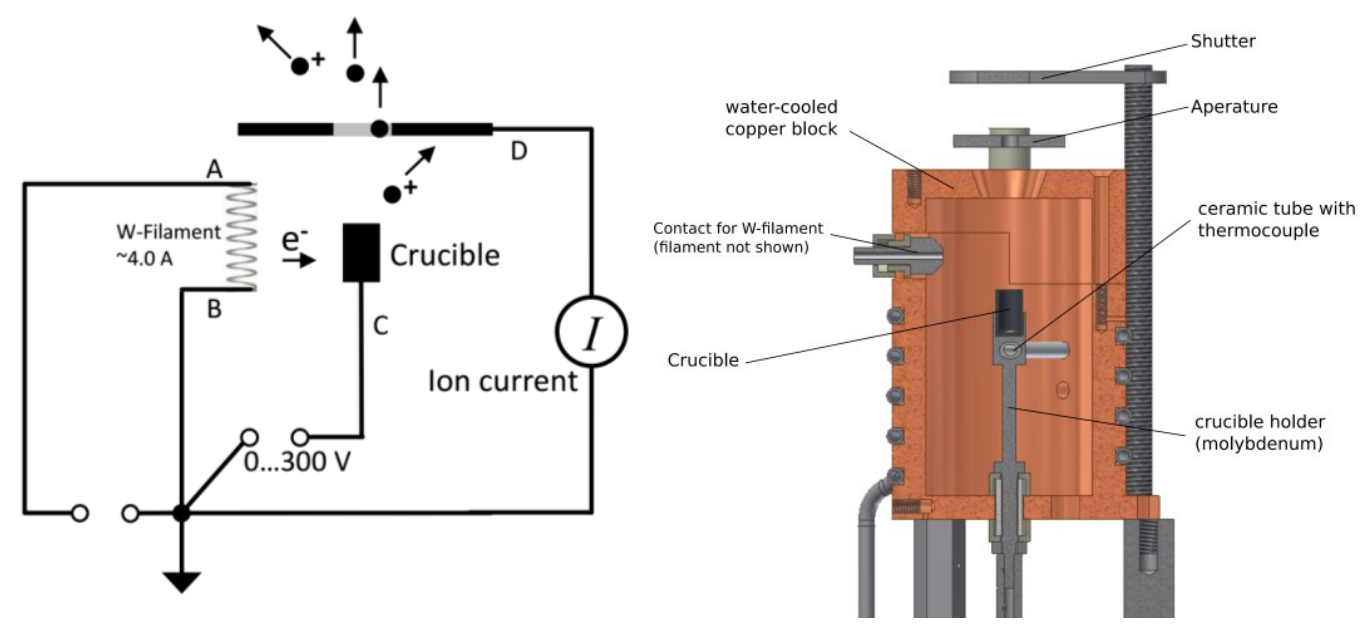

Figure 3.4: Left: Wiring diagram of the evaporator. Right: Cross section of 3D sketch.

The heart of the evaporator is a crucible which is made of glassy carbon, a conductable material stable at high temperatures with a fullerene-related microstructure (Sigradur ${ }^{\circledR}$, HTW HochtemperaturWerkstoffe $\mathrm{GmbH}$ ). Note that all components directly heated are made of a refractory material. The crucible is filled with silver pellets (1-3 mm diameter, $99.9999 \%$ purity Sigma Aldrich). The evaporation process is started by applying a current to the tungsten filament (contacts $A$ and B, Figure 3.4, left) and setting a positive voltage on the crucible (contact $\mathrm{C}$ ). As a result of the impact of the electrons, the crucible begins to heat up as can be read out by the thermocouple below the crucible (Figure 3.4, right). The material starts to melt and evaporates producing a beam of silver atoms unhampered in an UHV environment. A final aperture selects the part of the silver beam which is highly homogeneous and is 
employed for epitaxial growth of $\mathrm{Ag} / \mathrm{Au}$. Deposition time is controlled by opening and closing of the shutter.

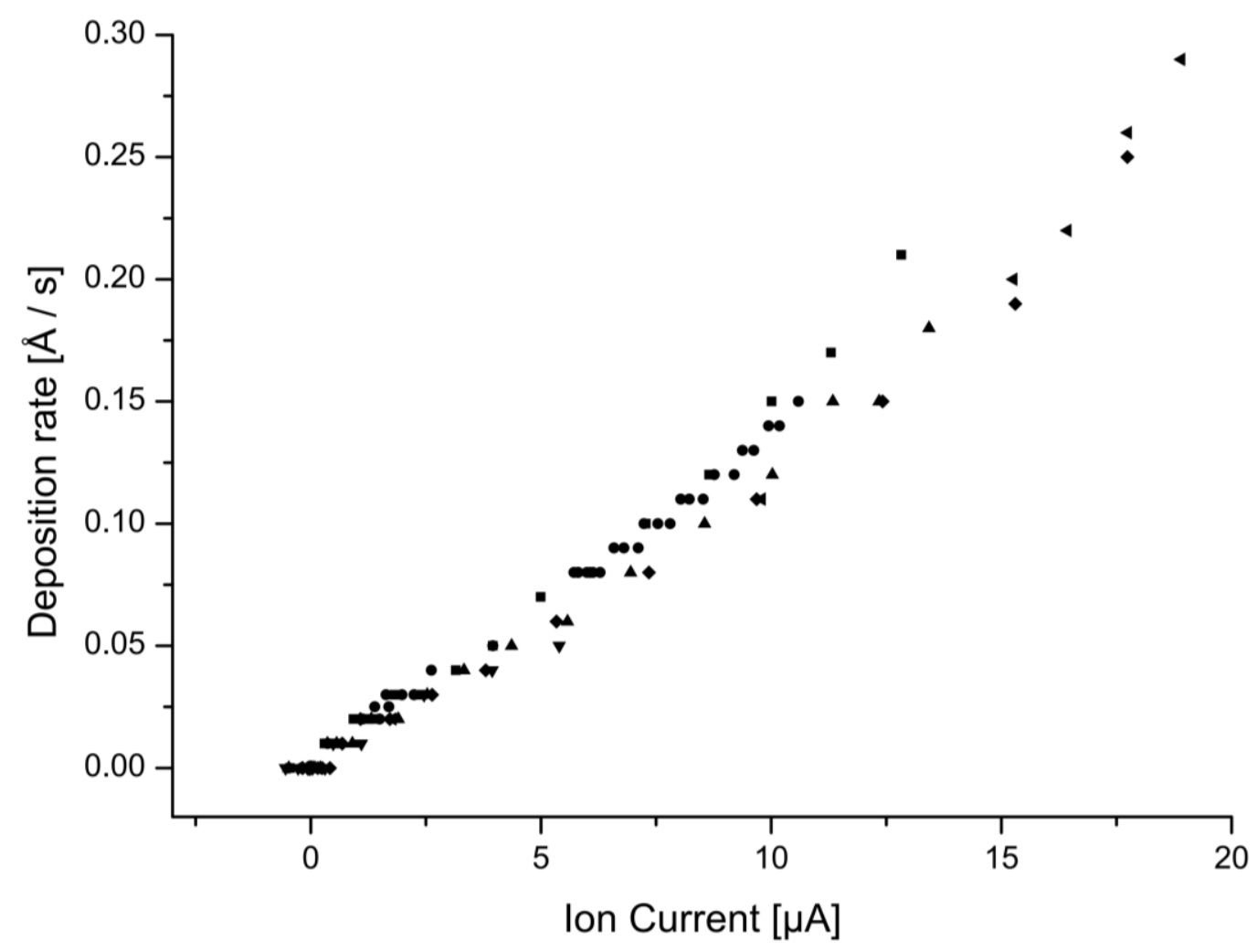

Figure 3.5: Deposition rate vs Ion Current. Overview of six different datasets with deviating parameters measured at different days shows high reproducibility.

The use of heating method based on electron bombardment offers an additional advantage: A small part of the evaporated silver atoms is ionized leading to a detectable ion current at the final aperature (contact D). The ion current is linearly correlated with the depositition rate, therefore it can be used as measure of the evaporation rate even when the shutter is closed (Figure 3.5). A complete set of performance curves of the evaporator are presented in Appendix A.

\section{Quartz Crystal Microbalance (QCM)}

The film deposition process is monitored by a Quartz Crystal Microbalance (QCM), a tool which working principle is presented in the current paragraph. Quartz is a piezoelectric material, that means applying a voltage generates a shear deformation, providing the means to determine the resonance frequency by electromechanical coupling. The frequency of a crystal in a QCM can be determined with very high precision and is lowered when additional mass is added to the face of the crystal. Most of the quartz crystals used for those applications are cut at an angle of $35^{\circ} 15^{\prime}$ from the Z-axis, a specification referred to as "AT cut".

Sauerbrey [78] first noted that the change of the frequency $\Delta f$ is linearly correlated with the change in mass $\Delta m$ from the added material. Based on that, first instruments used the Sauerbrey-equation to calculate the film thickness $T_{f}$ :

$$
T_{f}=K \cdot \frac{\Delta f}{\mathrm{~d}_{f}}
$$


The Sauerbrey constant is defined as $K=\frac{N_{A T} \cdot d_{q}}{f_{q}}$ with the frequency constant of an AT cut quartz $\left(N_{A T}=166100 \mathrm{~Hz} \mathrm{~cm}\right)$ and the density of a single crystal quartz $\left(d_{q}=2.649 \frac{\mathrm{g}}{\mathrm{cm}^{3}}\right)$. A crystal with a starting frequency of $f_{q}=6 \mathrm{MHz}$ will display a reduction of $8.59 \mathrm{~Hz}$ when 1.0 Angström of $\mathrm{Ag}\left(\mathrm{d}_{f}=\right.$ $\left.10.49 \frac{\mathrm{g}}{\mathrm{cm}^{3}}\right)$ is deposited. While the Sauerbrey equation is valid within the limitations of small frequency shifts (up to $\frac{\Delta f}{f_{q}}=0.02$ ), rigid films and evenly deposited mass (conditions that are approximately met in this work), a more sophistic formula is used today. This " $Z$ match technique" takes into account the acoustic properties of quartz and film by an additional factor which is the acoustic impedance ratio $Z$ [79]. Modern instruments like the one in the present work calculate the the film thickness according to that method which is in agreement with (3.1) but valid for even higher frequency shifts (to $\frac{\Delta f}{f_{q}}=0.4$ ). With the employment of the QCM, it is possible to monitor the film thickness and control the deposition rate to achieve best conditions for epitaxial growth of films of $\mathrm{Ag} / \mathrm{Au}(111)$.

\section{Calibration of the QCM}

To obtain the correct film thickness at the sample for the Ag/Au system, a calibration is necessary. At first, a simple estimation yields the thickness displayed by the QCM that corresponds to one monolayer of $\mathrm{Ag} / \mathrm{Au}(111)$. That value was estimated using simple geometrical considerations taking into account the distances and the angular distribution of the evaporator. Second, calibration is achieved experimentally by a measurement of Auger spectra at different thicknesses acquired by the QCM. The Auger Peak to Peak Height (APPH) of Au and Ag as a function of film thickness is expected to exhibit a kink after the completion of a monolayer (see section 2.3.3).
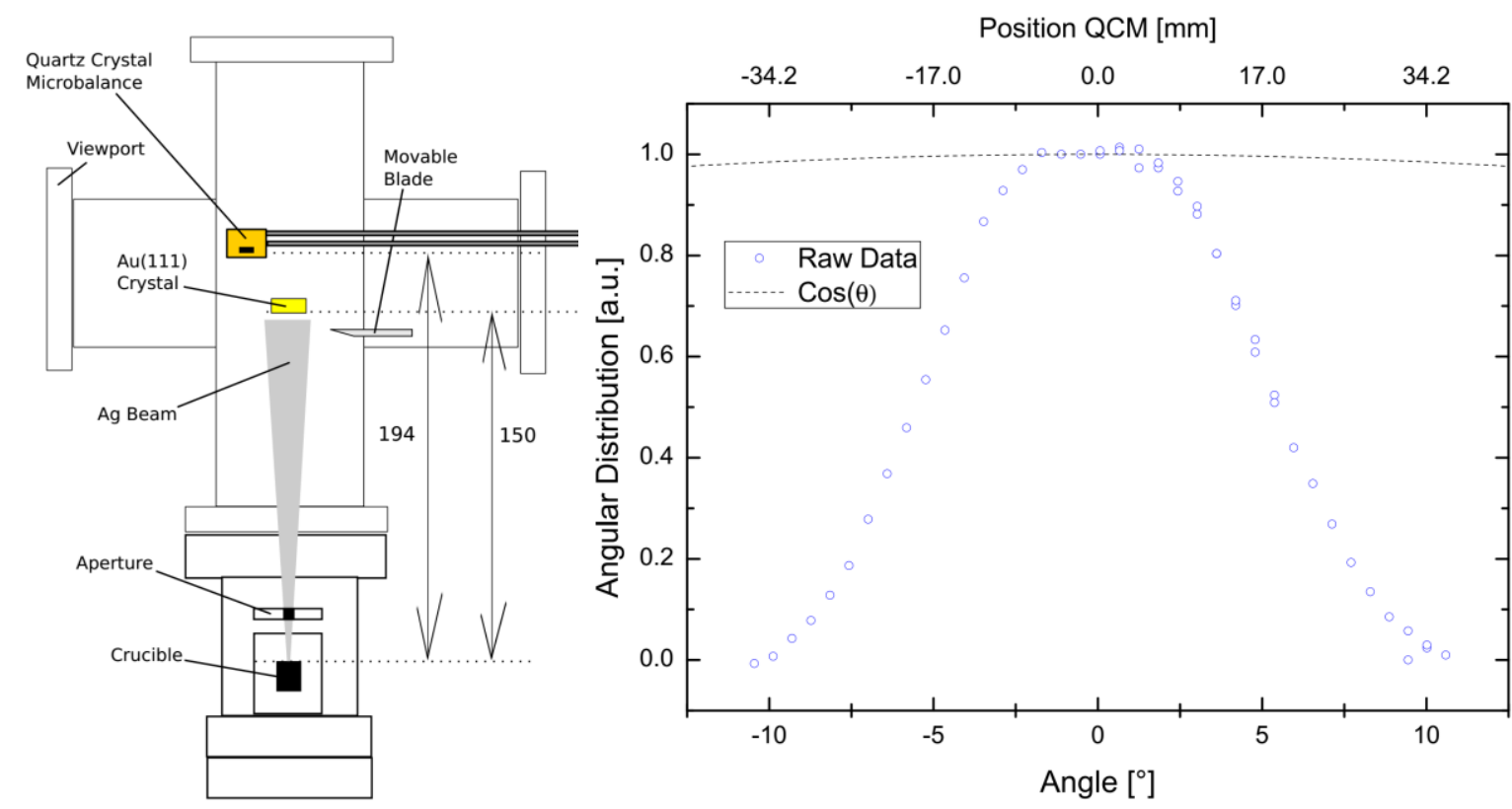

Figure 3.6: Left: Sketch of the deposition stage with distances. Right: Angular distribution of the Ag beam plotted against the angle (origin is the crucible opening) and the position of the QCM. With an ideal source, distribution described by $\cos (\Theta)$ (dashed line) is expected as achieved for the center for the data points (circles). In the center, a homogenous deposiiton is therefore achieved. Deviation is due to the final aperture reducing the coating of parts within the chamber to a minimum.

In Figure 3.6, the setup during the evaporation process in the test chamber is sketched with the distances of the $A u(111)$ crystal and the QCM to the crucible opening. The QCM is connected to a movable flange and its position can be shifted up to $36 \mathrm{~cm}$ distance to the center. For the deposition, it was set to the depicted position $\left(10 \mathrm{~cm}\left(\sim 3^{\circ}\right)\right.$ shifted from the center to the viewport). In this 
configuration, the QCM can monitor the deposition process because the Au crystal is not blocking the silver beam. By moving the QCM, the angular distribution can be obtained and is depicted in Figure 3.6 (right). A few degrees around the center, a homogeneous deposition region is detected originating from an unperturbed source which is ideally described as a cosine distribution (dashed line). Due to the aperture at the end of the evaporator, which reduces coating of other parts within the chamber, a narrower distribution obtained.

The simplest model is the assumption of the crucible as a point source producing a silver beam with the shape of a spherical cap. At distance $d$, the area of the spherical cap can be expressed as

$$
A=2 \pi d^{2}(1-\cos \vartheta)
$$

with the half angle $\vartheta$ of the cone. Taking the ratio of the areas at two distances from the source $d_{1}$ and $d_{2}$ we arrive at

$$
\frac{A_{1}}{A_{2}}=\frac{2 \pi d_{1}^{2}(1-\cos \vartheta)}{2 \pi d_{2}^{2}(1-\cos \vartheta)}=\frac{d_{1}^{2}}{d_{2}^{2}}
$$

The thickness $T$ of a film deposited is inversly proportional to the area upon which it is deposited:

$$
T=\frac{1}{A}
$$

Finally, combining (3.3) and (3.4) the thickness produced by the beam at two different distances can be calculated by

$$
\frac{\mathrm{T}_{1}}{\mathrm{~T}_{2}}=\frac{\mathrm{d}_{2}^{2}}{\mathrm{~d}_{1}^{2}}
$$

Due to the slight shift of the QCM position $\left(10 \mathrm{~cm}, \sim 3^{\circ}\right)$ a correction factor of $f_{Q C M}=0.93$ as determined from the angular distribution needs to be applied in the final calculation.

Thus, the thickness of one monolayer of $\mathrm{Ag} / \mathrm{Au}(111)$ which reported as $T_{A g / A u}=2.36 \AA$ [80] leads to an estimated QCM readout of

$$
\mathrm{T}_{\mathrm{QCM}}=\frac{\mathrm{T}_{1}}{\mathrm{~T}_{2}} \cdot \mathrm{f}_{\mathrm{QCM}} \cdot \frac{\mathrm{T}_{\overline{\mathrm{Ag}}}}{\mathrm{Au}}=1.3 \AA
$$

It is therefore expected to obtain a calibration value close to that thickness which will be confirmed by the following calibration measurement.

Figure 3.7 shows the Auger spectra of a clean $\mathrm{Au}(111)$ and $\mathrm{Ag} / \mathrm{Au}(111)$ films at two different thicknesses. The most characteristic peak in the first spectrum (black line) is the Au peak at $69 \mathrm{eV}$. With $\mathrm{Ag}$ on the surface, its characteristic double peak at $356 \mathrm{eV}$ can be observed. The peak intensities or Auger Peak to Peak Heights (APPH) of the $\mathrm{Au}[\mathrm{Ag}$ ] peak decrease [increase] with increasing film thickness. 


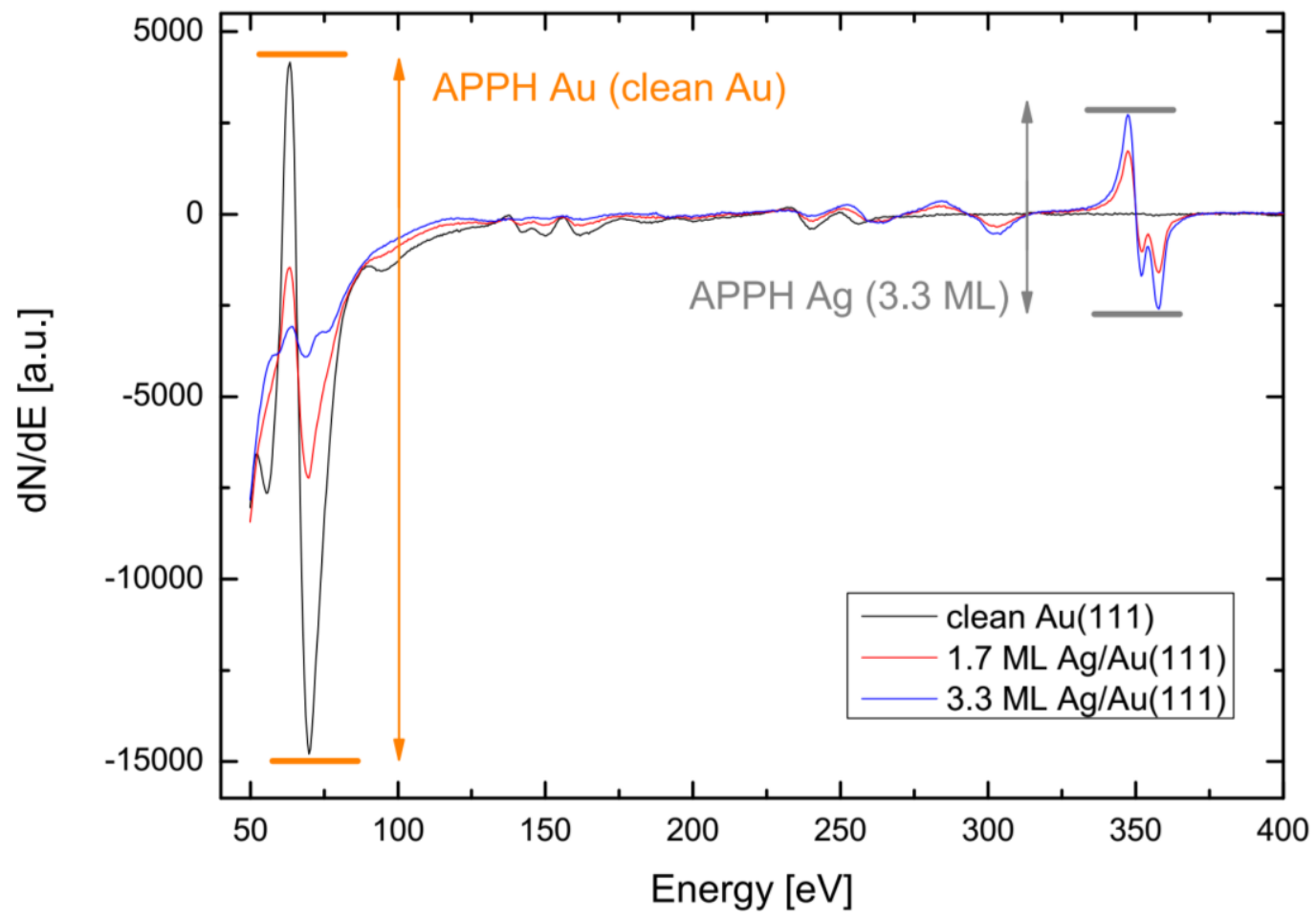

Figure 3.7: Auger Spectra of clean Au(111), 1.7 ML Ag/Au(111) and 3.3 ML Ag/Au(111). The APPH of Au from a clean Au(111) and $\mathrm{Ag}$ from 3.3 $\mathrm{ML} \mathrm{Ag/Au(111)} \mathrm{are} \mathrm{depicted} \mathrm{in} \mathrm{orange} \mathrm{and} \mathrm{grey.} \mathrm{The} \mathrm{APPH} \mathrm{of} \mathrm{Au}$ [Ag] decrease [increase] with increasing film thickness.

Before deposition, the crystal is cleaned by Ar sputtering $(30 \mathrm{~min})$ and subsequent annealing $\left(700^{\circ} \mathrm{C}\right)$ resulting in a clean surface as seen in the Auger spectrum (Figure 3.7, black line). To avoid mixing of substrate and adsorbate, deposition is started when the sample had cooled below $50^{\circ} \mathrm{C}$.

For the calibration measurement, the $\mathrm{Au}(111)$ crystal is subsequently coated with $\mathrm{Ag}$ in small steps. The evaporator is heated until a low but detectable and stable deposition rate is reached as monitored with the QCM. After every deposition, the AES was measured and the normalized APPH of the Au and $\mathrm{Ag}$ were plotted against the thickness as measured by the QCM.

A statistical model was employed to fit the data by two connected straight lines with different slopes. It contained four parameters: the y-intercept $b$, the slopes of the straight lines $m_{1}$ and $m_{2}$ and the breakpoint $L$. The break point $L$ was varied, all other parameters were determined by fitting the model to the data. When the root-mean-square error (RMSE), a minimum was found for both data sets at with a break point at $1.2 \AA$. This value is close to the estimation assuming the simple point source model (equation 3.6) and was chosen as calibration value. Using that value, the low but previously unknown deposition rate turned out to be $\sim 1.0 \mathrm{ML} / \mathrm{min}$ which is a typical rate used in the preparation of $\mathrm{Ag} / \mathrm{Au}(111)$ films $[10,12]$ and will be used for all further experiments.

The APPH of Au and Ag is plotted against the calibrated thickness and depicted in Figure 3.8. 


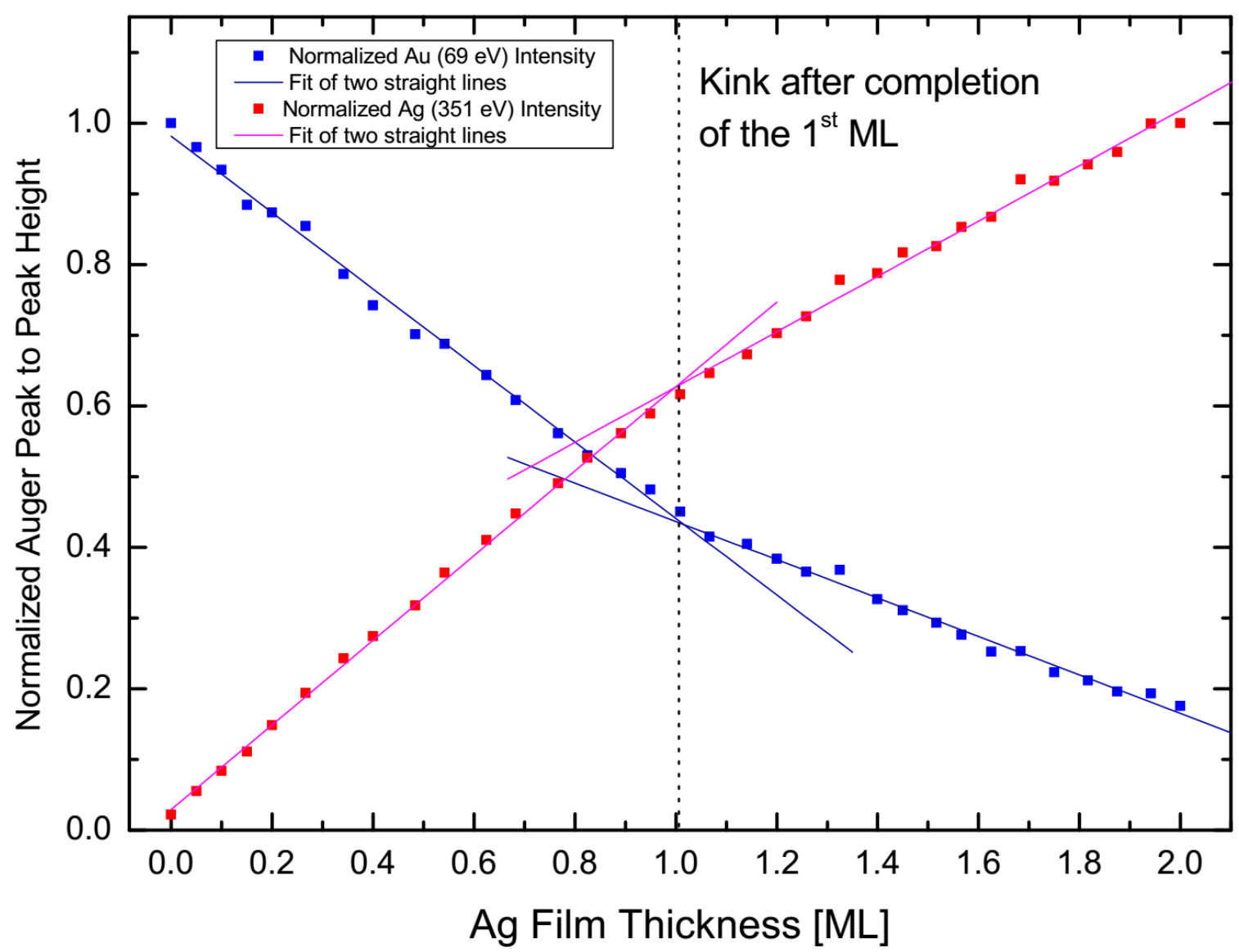

Figure 3.8: Normalized APPH of Au (69 eV) and Ag (356 eV) plotted against the Ag film thickness (after calibration). In both signals, a linear trend can be observed with a break after formation of the first monolayer.

In a second measurement, spectra were acquired with a lower point density but broader range of thicknesses. Analogous to the first dataset, a model consisting of five subsequent straight lines was assumed. The break point $L$ was varied from 0.7 to $1.5 \AA$ and the RMSE were compared. In comparison to the fitting procedure presented above, this method is rather prone to errors due to a lower point density per line and rather flexible model with a higher number of fitting parameters. Additionally, the kinks in the trend are less pronounced with increasing film thickness. Still, an absolute minimum in the RMSE was reached with the previously determined breakpoint of $1.2 \AA$ when fitting the model to the Au data. Using the experimentally determined calibration value and plotting data and model against the thickness (after calibration) we obtain Figure 3.9 which provides the best way to visualize the breaks after layer completion up to the $4^{\text {th }} \mathrm{ML}$ confirming the layer-by-layer growth as expected for $\mathrm{Ag} / \mathrm{Au}(111)$. 


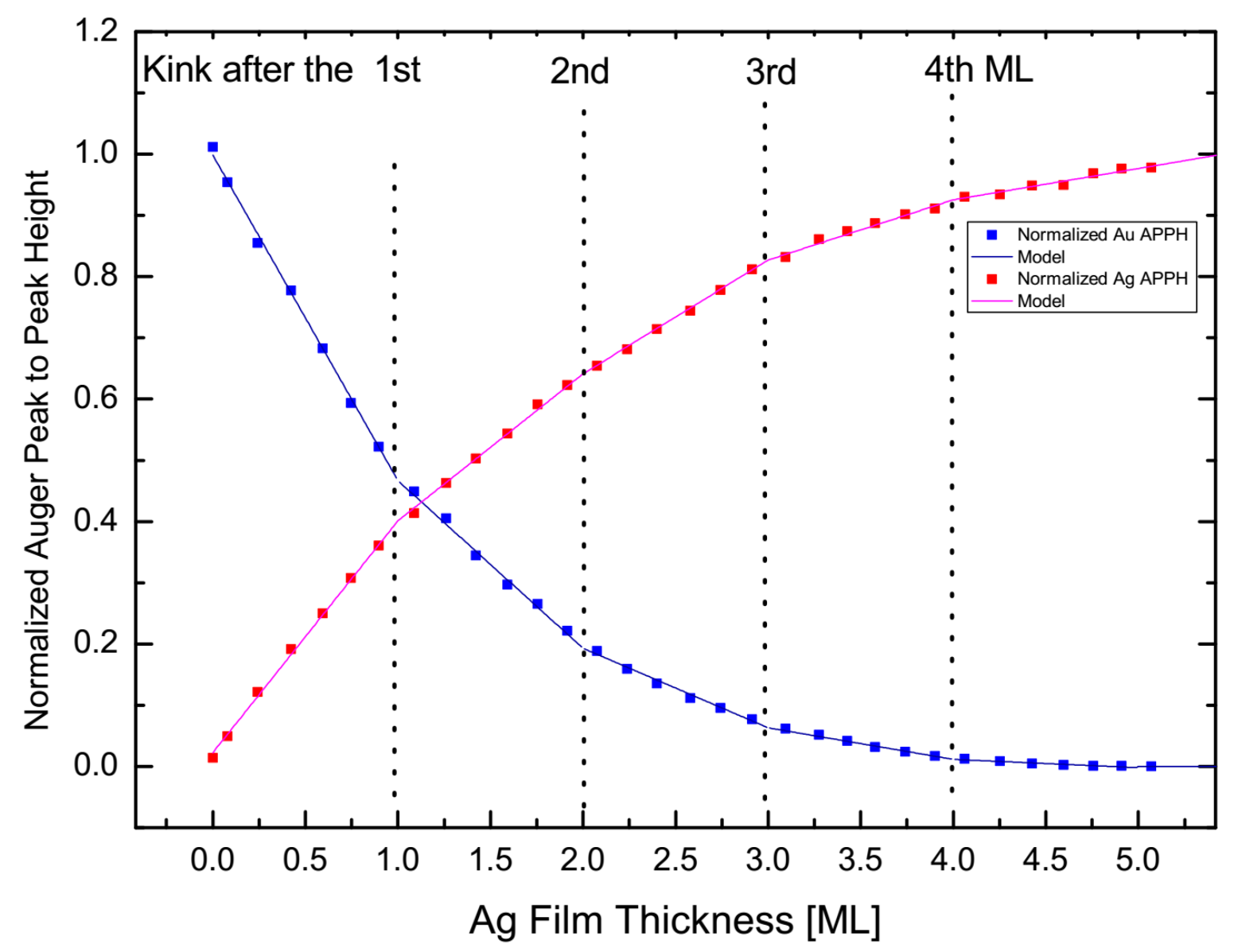

Figure 3.9: Normalized APPH of Au (69 eV) and Ag (356 eV) plotted against the film thickness (after QCM calibration). A model of five subsequent straight lines with the previously determined calibration value is fitted to each data set for visualization of the kinks after completion of a monolayer of Ag/Au(111).

\subsubsection{Film Characterization}

\section{Thickness Determination by AES}

AES is a method that can be used for quantitative purposes. While the absolute APPH of a single peak can vary from day to day, the ratio of two different peaks is a reproducible measure. From Figure 3.9, it can be seen that the signal of the substrate Au can be detected up to a thickness of about $4 \mathrm{ML}$. With the knowledge of the calibration value (see previous section), up to that thickness, the present data of $\mathrm{Au}$ and $\mathrm{Ag} \mathrm{APPH}$ can be used to determine the thickness of a $\mathrm{Ag} / \mathrm{Au}(111)$ film from the Auger spectrum. The Ag/Au APPH ratio was calculated and plotted as a function of film thickness. Figure 3.10 shows two measurements from different days acquired at the test chamber showing high reproducibility. A third measurement acquired after mounting the evaporation unit to the molecular beam scattering machine ("Beamer I") is presented as well. Due to the high reproducibility, it is estimated that the thickness measurement by Auger can be performed with an thickness precision of $\pm 0.1 \mathrm{ML}$. 


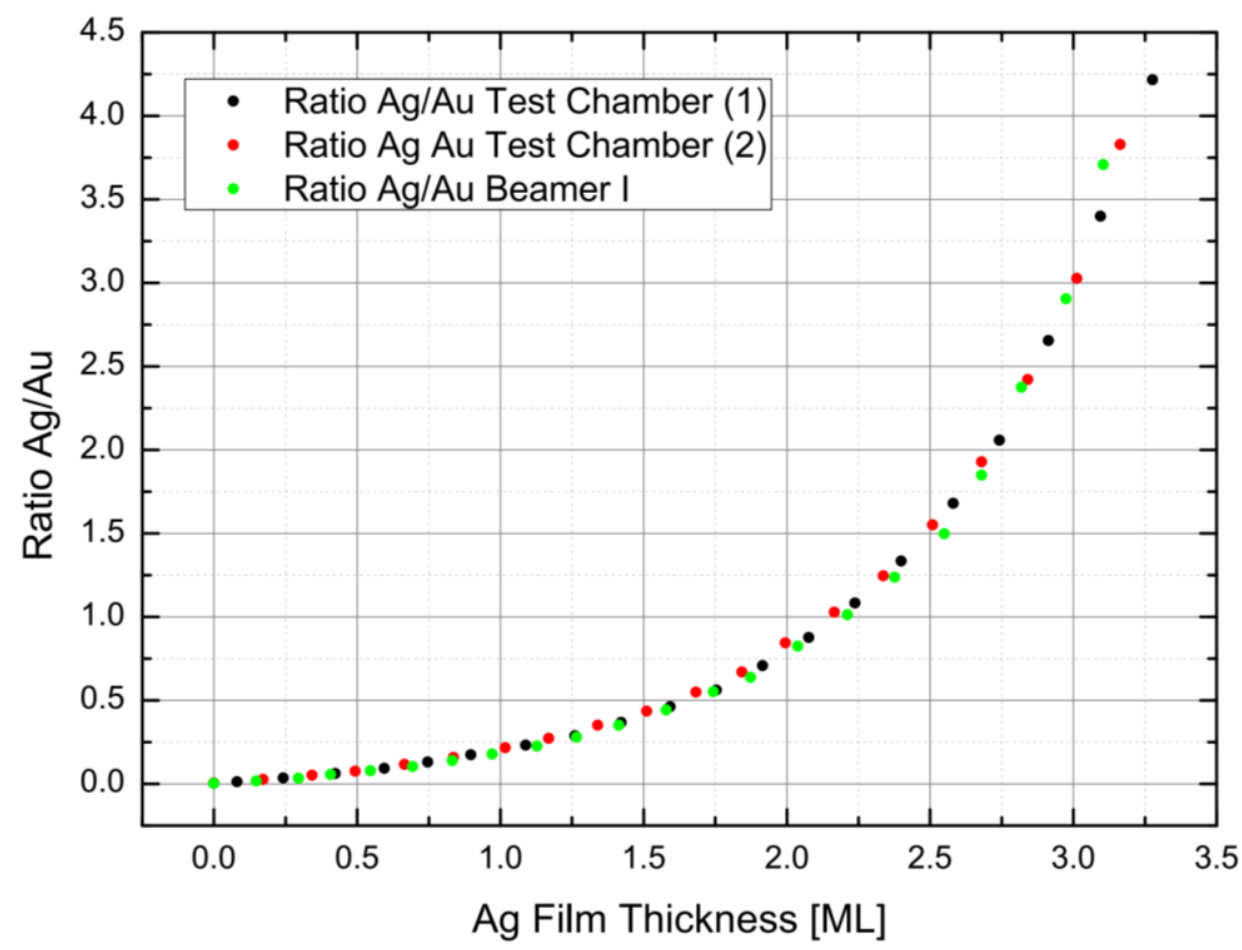

Figure 3.10: APPH Ratio of Ag/Au vs Ag film thickness. Beside two measurements acquired at the test chamber, a measurement at the molecular beam scattering machine ("Beamer I") is depicted.

\section{Thermal Stability}

While the thin film system $\mathrm{Ag} / \mathrm{Au}$ exhibits ideal layer-by-layer growth due to the negligible lattice mismatch, both metals are prone to form alloys at elevated temperature. Similar to measurements by Meinel et al. [57] on the $\mathrm{Au} / \mathrm{Ag}(111)$ system, thermal stability measurements have been performed on $\mathrm{Ag} / \mathrm{Au}$ surfaces in this work.

After standard cleaning procedures, the crystal is coated homogeneously with $1.7 \mathrm{ML} \mathrm{Ag} / \mathrm{Au}(111)$ at room temperature $(300 \mathrm{~K})$. The temperature is then stabilized to the desired range and every $30 \mathrm{~min}$ a $A E S$ is taken at the center of the sample. As a measure of stability, the substrate/adsorbate ( $\mathrm{Au} / \mathrm{Ag})$ ratio of the AES intensities is determined. Please note that this APPH ratio is explicitly the inverse of the ratio discussed in the previous paragraph: Here, the APPH of the substrate (Au) is devided by the adsorbate (Ag). If any diffusion occurs, the adsorbate signal decreases and at the same time the substrate signal is expected to increase, leading in total to an increase of the substrate/adsorbate ratio. The substrate/adsorbate ratios were normalized to the initial value at annealing time $t=0 \mathrm{~min}$. The results are plotted in Figure 3.11 in comparison with two data sets from the previously mentioned data of $1.0 \mathrm{ML} \mathrm{Au} / \mathrm{Ag}$ at 30 and $101^{\circ} \mathrm{C}$ [57].

Within the limits of the experiment, the sample of $1.7 \mathrm{ML} \mathrm{Ag} / \mathrm{Au}(111)$ is stable in the range of $30-70^{\circ} \mathrm{C}$ (black boxes and red cirlces) over three hours. This is comparable to the data set of the Au/Ag sample at $30^{\circ} \mathrm{C}$. But at higher temperatures $\left(70-90^{\circ} \mathrm{C}\right)$, an increase of the substrate/adsorbate ratio can already be observed.

As a consequence, to avoid any change of the sample, the temperature for all subsequent experiments was kept close to room temperature. 


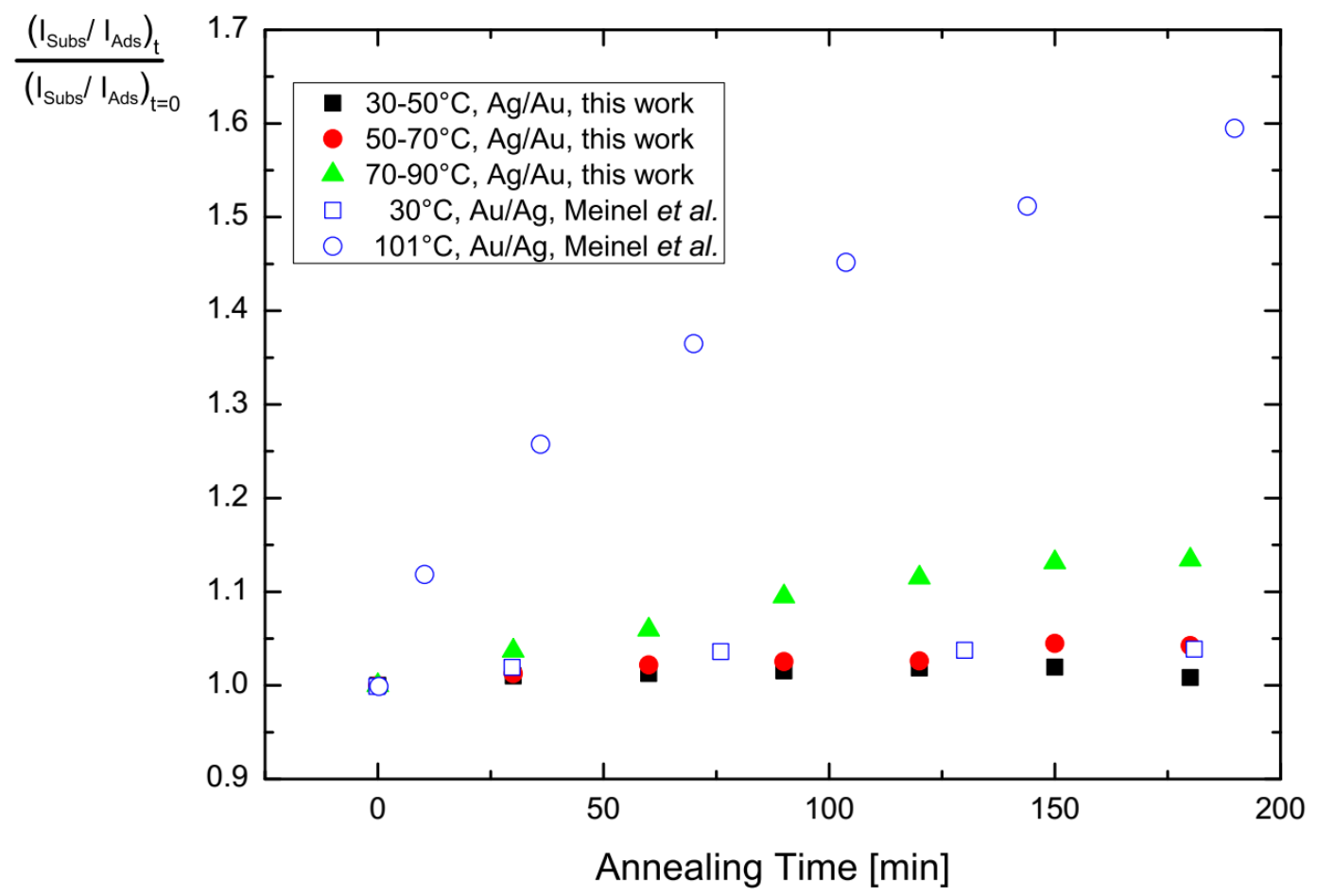

Figure 3.11: The aging of a $1.7 \mathrm{ML}$ film Ag/Au(111) investigated by AES. The APPH ratio of substrate (Au) by adsorbate (Ag) is plotted against the annealing time (normalized to the initial value). For comparison, data from a previous study of $1.0 \mathrm{ML}$ Au/Ag is shown as well.

\section{Homogeneity}

One important requirement of thin films is that they coat the substrate uniformly. For the identification of thickness-dependent effects, it is necessary to ensure full control over this experimental parameter. The $\mathrm{Au}(111)$ crystal is a two-dimensional surface, thus homogeneity can be measured along both axes referred to as $X$ and $Z$ (see Figure 3.12). Note that the results shown here were measured in the molecular beam scattering machine (described in section 3.2 ).

After deposition of a silver film with 1.7 ML thickness on the clean $\mathrm{Au}(111)$ at room temperature, both axes are scanned by AES along the center. The Ag/Au APPH ratio is converted into film thickness (see Figure 3.10) and plotted against the sample position (Figure 3.13, black boxes). Additionally to the total film thickness, the relative deviation from the mean value is shown (dashed line). 


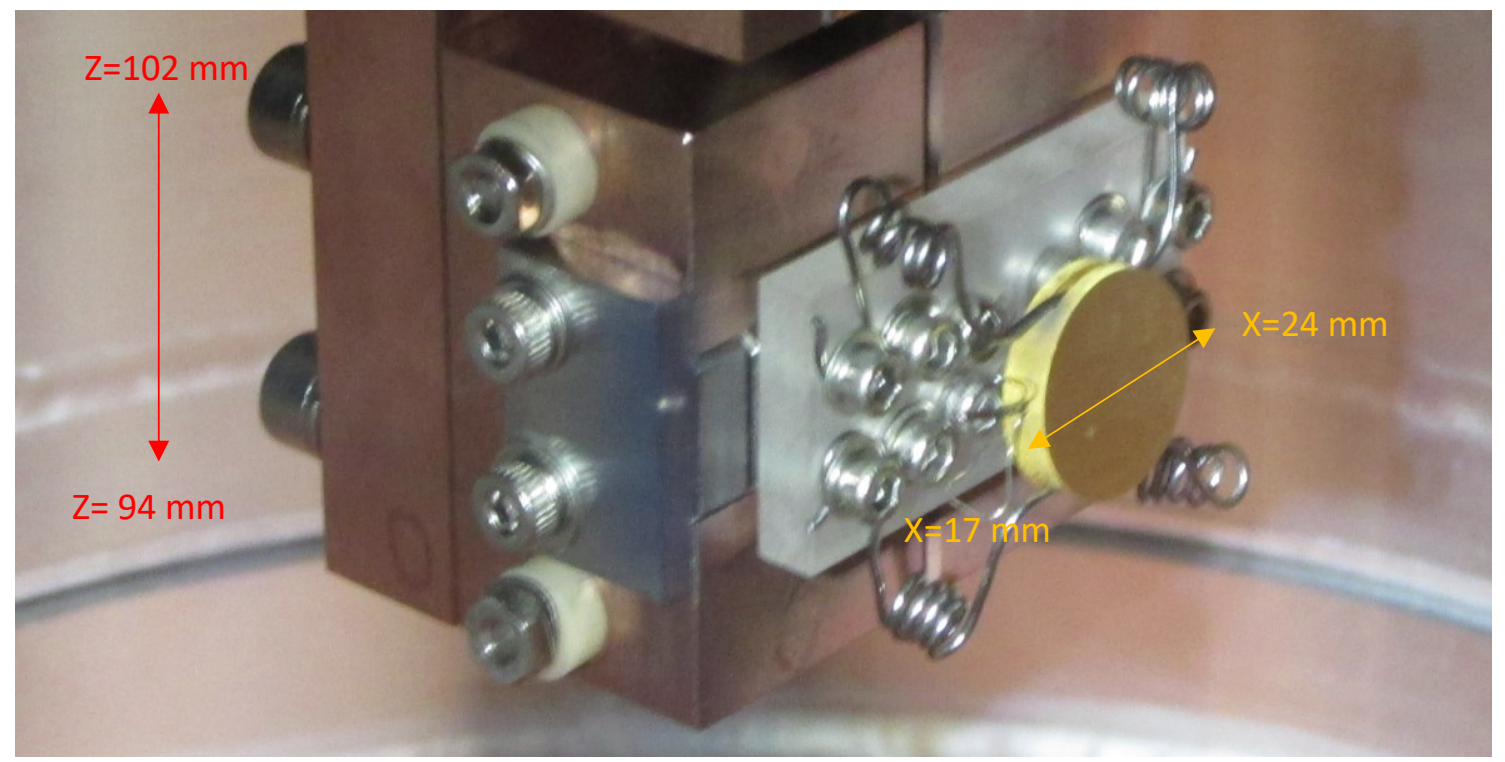

Figure 3.12: Homogeneity measurement along both axes of the Au(111) crystal in Beamer I. For a better orientation, the outmost manipulator positions of the homogeneity measurement (Figure 3.13) are noted.
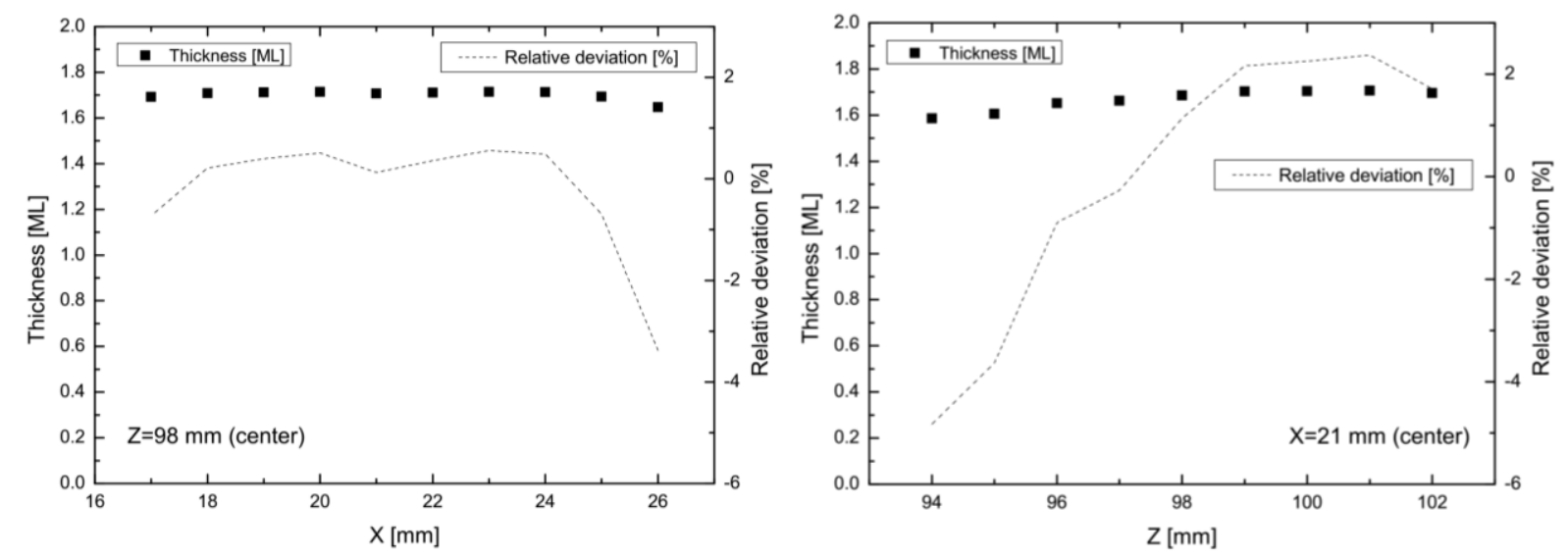

Figure 3.13: Homogeneity measurements along X (left) and Z (right) axis obtained by AES scans. Beside the absolute thickness (black boxes, left y axis scale), the relative deviation to the mean value is shown (dashed line, right y axis scale).

We observe that very good homogeneity can be accomplished. From Figure 3.13 (left panel), the center is homogeneous with deviations less than $1 \%$. The $Z$ axis exhibits slightly higher deviations from the mean value (Figure 3.13, right panel), the strongest deviation is about $5 \%$ at the lower part of the crystal. In the following molecular beam scattering experiments, a movable mask blocking the silver beam will be employed to grow a thickness gradient along the crystal (details in 3.2.3). The corresponding axis will be the $Z$ axis. For the characterization of the film thickness, AES scans will be performed at the center of the crystal $(X=21)$ along the $Z$ axis. Due to the excellent homogeneity along the $X$ axis, a thickness value measured at a certain $Z$ position is assumed to be equal for any $X$ position. 


\subsection{Molecular Beam Scattering Experiments}

\subsubsection{UHV Chamber for Molecular Beam Scattering (Beamer I)}

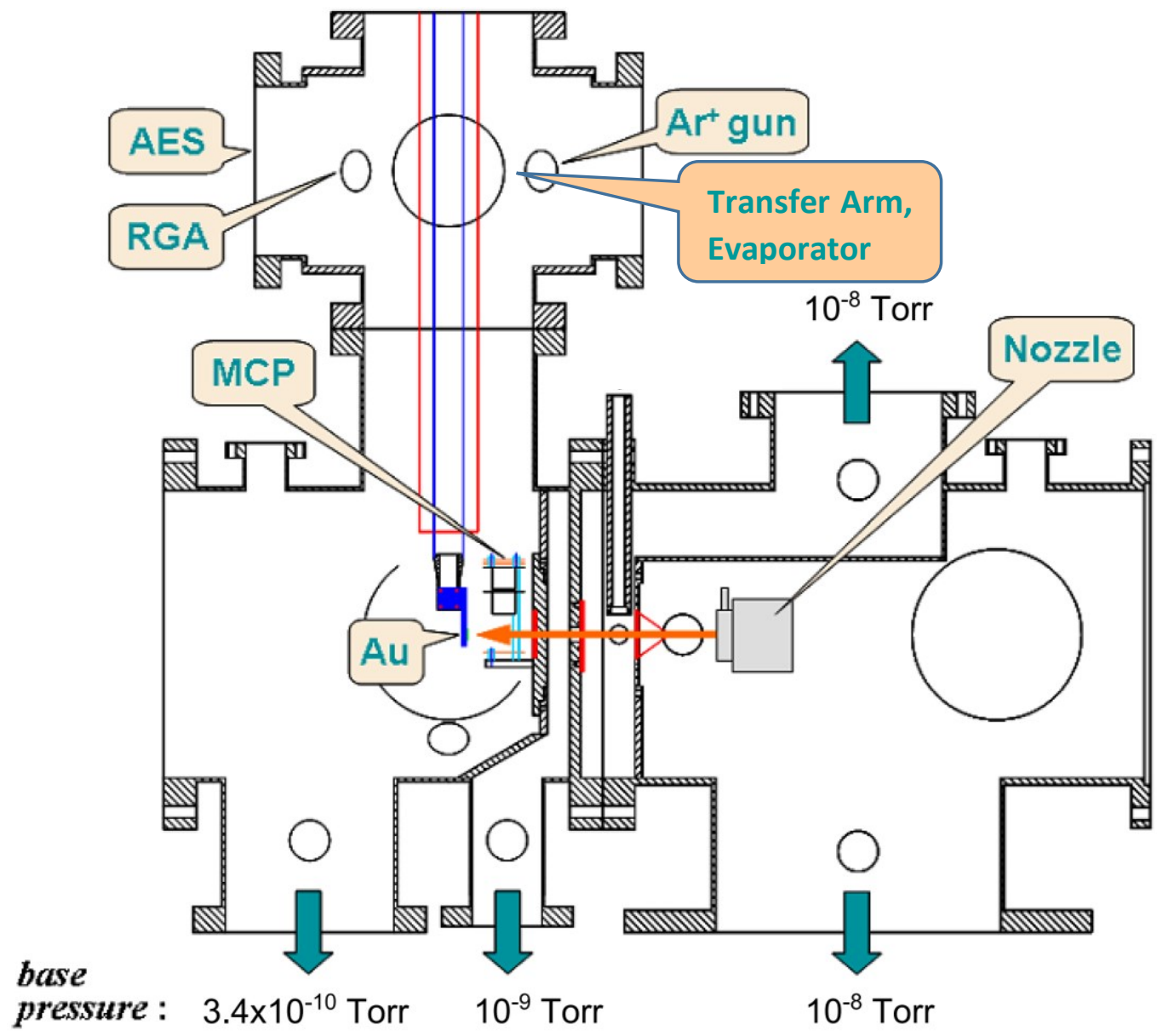

Figure 3.14: Basic Setup of the UHV chamber used in the molecular beam scattering experiments. Cleaning, preparation and cleaning of the surface is carried out in the top part of the chamber, the molecular beam is produced in the bottom part collides with the (modified) Au-surface. Abbreviations: AES (Auger Electron Spectrometer), RGA (Residual Gas Analyzer), MCP (Multi Channel Plate). The pressures are given for the case of no nozzle operation.

The molecular beam scattering experiments of the present work are carried out in a modified UHV chamber which is described elsewhere [81], thus only a short description will be provided here. In gassurface scattering experiments, it is crucial to maintain good vacuum conditions to avoid modifications of the investigated surface for the sake of reproducibility. Special care has to be taken to reach base pressures of low $10^{-10}$ Torr.

The apparatus is depicted in Figure 3.14. It consists of four interconnected, differentially pumped chambers, referred to (from right to left) as source chamber, first differential chamber, second differential chamber, surface chamber. This design allows the production of very intense molecular beams and the maintenance of good vacuum conditions in the surface chamber at the same time. The source chamber is pumped by a turbo molecular pump (TMP) (Edwards STP-A2203C). The general advantage of this new pump is that in comparison to the previous setup which employed a cryogenic pump, now it is possible to use Helium as a carrier gas (although not employed in this work). Although the pumping rate for $\mathrm{H}_{2}$ is slightly lower $(1700 \mathrm{~L} / \mathrm{s}$ vs. $3000 \mathrm{~L} / \mathrm{s})$, the same pressure of $\sim 5 \times 10^{-6} \mathrm{Torr}$ is achieved when the nozzle is operating. First and second differential stage are pumped by TMPs (Seiko Seiki STP-400 and Osaka TF160CA) with pumping speeds of $400 \mathrm{~L} / \mathrm{s}$ and $120 \mathrm{~L} / \mathrm{s}$. The second differential 
stage can be separated from the first stage and the source chamber by a slide valve allowing to vent the high pressure part without breaking the vacuum in the surface chamber. The surface chamber with the best vacuum conditions is pumped by a TMP (Leybold NT360, $350 \mathrm{~L} / \mathrm{s}$ ), backed by a booster pump (Pfeiffer-Balzers, TPU062, $70 \mathrm{~L} / \mathrm{s}$ ). Cleaning, preparation and analysis of the sample is carried out in the top part of the chamber which is equipped with an Ar lon Gun (LK Tech) and an Auger (STAIB ESA100). The transfer arm with the evaporator stage was attached to a free flange is mounted to that part of the chamber as well. A 4 axes $(x, y, z, \phi)$ UHV manipulator (Vacuum Generator Fisons Instruments, Omniax 800) transports the sample between the top and bottom part of the chamber.

In the source chamber, a molecular beam is produced by supersonic expansion of the sample gas mixture [82]. Supersonic expansion is achieved, when a high pressure gas is expanded through a small orifice into vacuum. Due to many collisions at the opening, the gas loses most of its vibrational and rotational energy. Furthermore, this process results in a narrow velocity distribution. For an ideal gas mixture, the most probable speed is given by

$$
\bar{v}_{\infty}=\sqrt{\frac{2 \cdot \bar{C}_{p}}{\bar{M}} \cdot T_{0}}
$$

with stagnation gas temperature $T_{0}$, the average heat capacity $\bar{C}_{p}$ and the average molar mass $\bar{M}$. By seeding the sample gas with an inert and light carrier gas (typically $\mathrm{H}_{2}$ ), the average mass can be lowered increasing the most probable speed of the molecular beam. With the seeding strategy, a wide range of velocities is available to the experimentalist. As an example, in the experiments discussed in chapter 4, gas mixtures with $7 \%$ and $2 \% \mathrm{NO}_{\text {in }} \mathrm{H}_{2}$ are prepared with a mean kinetic energy of $0.58 \mathrm{eV}$ and $0.95 \mathrm{eV}$. On the other hand, gas mixtures employed in chapter 4.1 .3 and 6 are prepared in concentrations of $6 \%$ and $2 \% \mathrm{CO} / \mathrm{H}_{2}$ with $0.64 \mathrm{eV}$ and $0.92 \mathrm{eV}$.

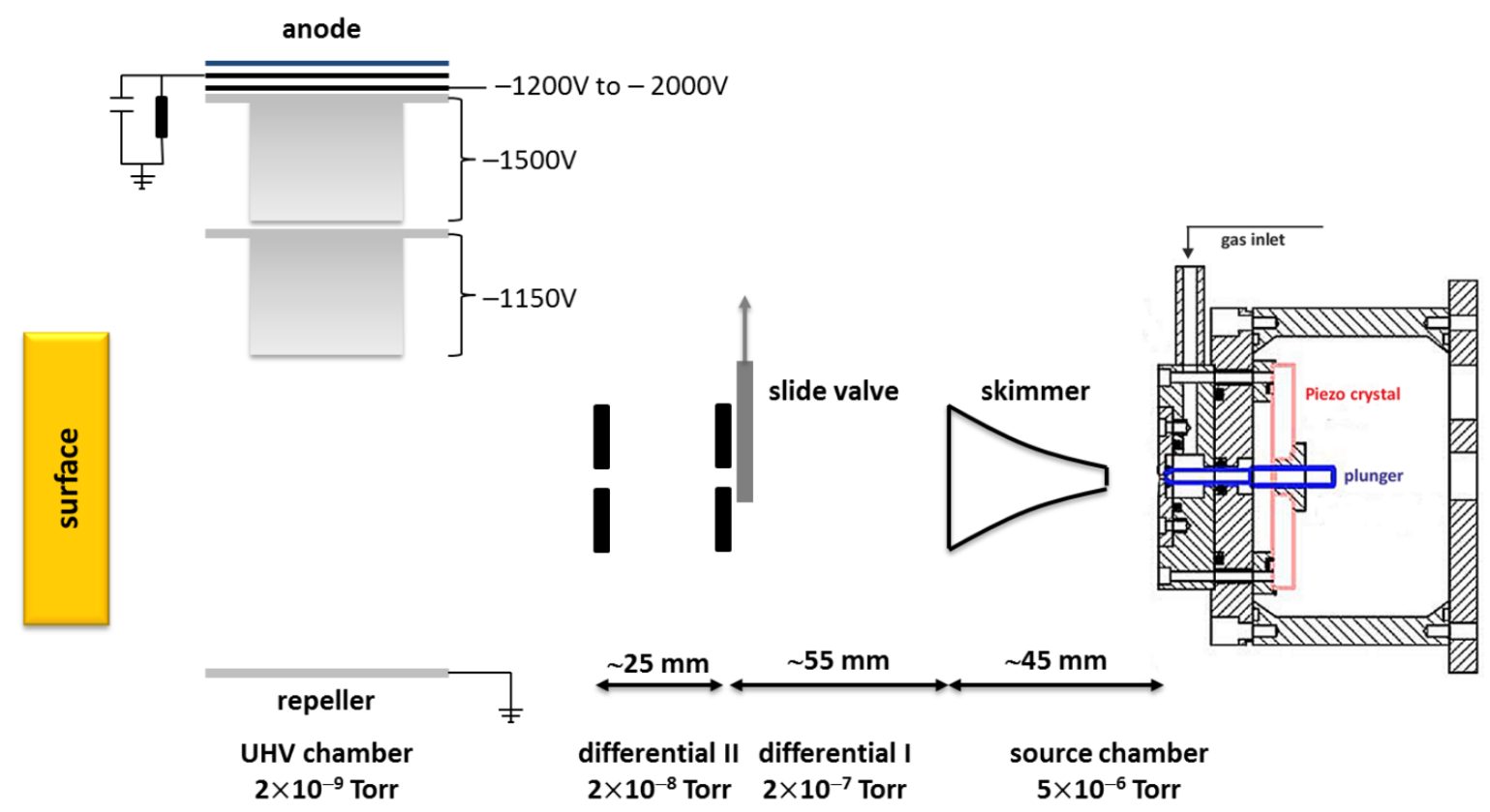

Figure 3.15: Path of the molecular beam from the nozzle (right) to the surface. Pressures are given for the operating nozzle.

The path of the molecular beam is sketched in Figure 3.15 (taken from [49]). A pulsed molecular beam $(10 \mathrm{~Hz})$ is generated by supersonic expansion (stagnation pressure of $3 \mathrm{bar}$ ) through a piezo-driven nozzle (orifice diameter of $1 \mathrm{~mm}$ ). Opening of the valve is achieved with a piezo crystal biased with a $70 \mu \mathrm{s}$ pulse at $460 \mathrm{~V}$. After passing a skimmer $(1.5 \mathrm{~mm})$ selecting the inner part, the beam passes two differential pumping stages separated by a $2 \mathrm{~mm}$ aperture. Finally, it enters the surface chamber 
through a $3 \mathrm{~mm}$ aperture. The sample gas is probed by resonance enhanced multiphoton ionization (REMPI) via a laser and subsequent detection of the ions. With the manipulator/surface and repeller are grounded to define a suitable electric field. The two metal cylinders are bias to $-1150 \mathrm{~V}$ and $-1500 \mathrm{~V}$ respective to work as an ion lens guiding ions to a chevron multi-channel plate detector.

The advantage of this chamber design is that due to the short distance between nozzle and sample $(\sim 180 \mathrm{~mm})$, molecular beams of high intensity and low time spread can be produced. As a result, when performing a TOF scan of the molecular pulse in the UHV chamber, short beam pulses with a FWHM of 30-50 $\mu$ s are detected. Additionally, the differential pumping design with the pulsed nozzle allows to maintain low pressures of $\sim 2 \times 10^{-9}$ Torr in the surface chamber with the molecular beam on.

\subsubsection{State-Specific Detection and Excitation of Molecules}

For the state-specific detection of molecules, resonance-enhanced multiphoton ionization (REMPI) is employed. In its simplest case, a molecule is undergoing two-photon ionization: After adsorption of one photon and excitation to an intermediate state, it adsorbs a second photon leading to further excitation and overcoming of the ionization barrier. In the general case of multiphoton ionization, the intermediate state is not an eigenstate of the system, therefore this process is only of limited probability. However in REMPI, it gets strongly enhanced because the intermediate state involved in the first adsorption step is an existing electronic state of the molecule. Due to the highly efficient detection of the resulting ions, REMPI is one of the most sensitive detection techniques. Good conditions are achieved with an intense tunable laser able to produce the UV photons of suitable wavelength, a narrow bandwidth which allows quantum state resolution and a strong multiphoton adsorption cross section for the chosen transition.

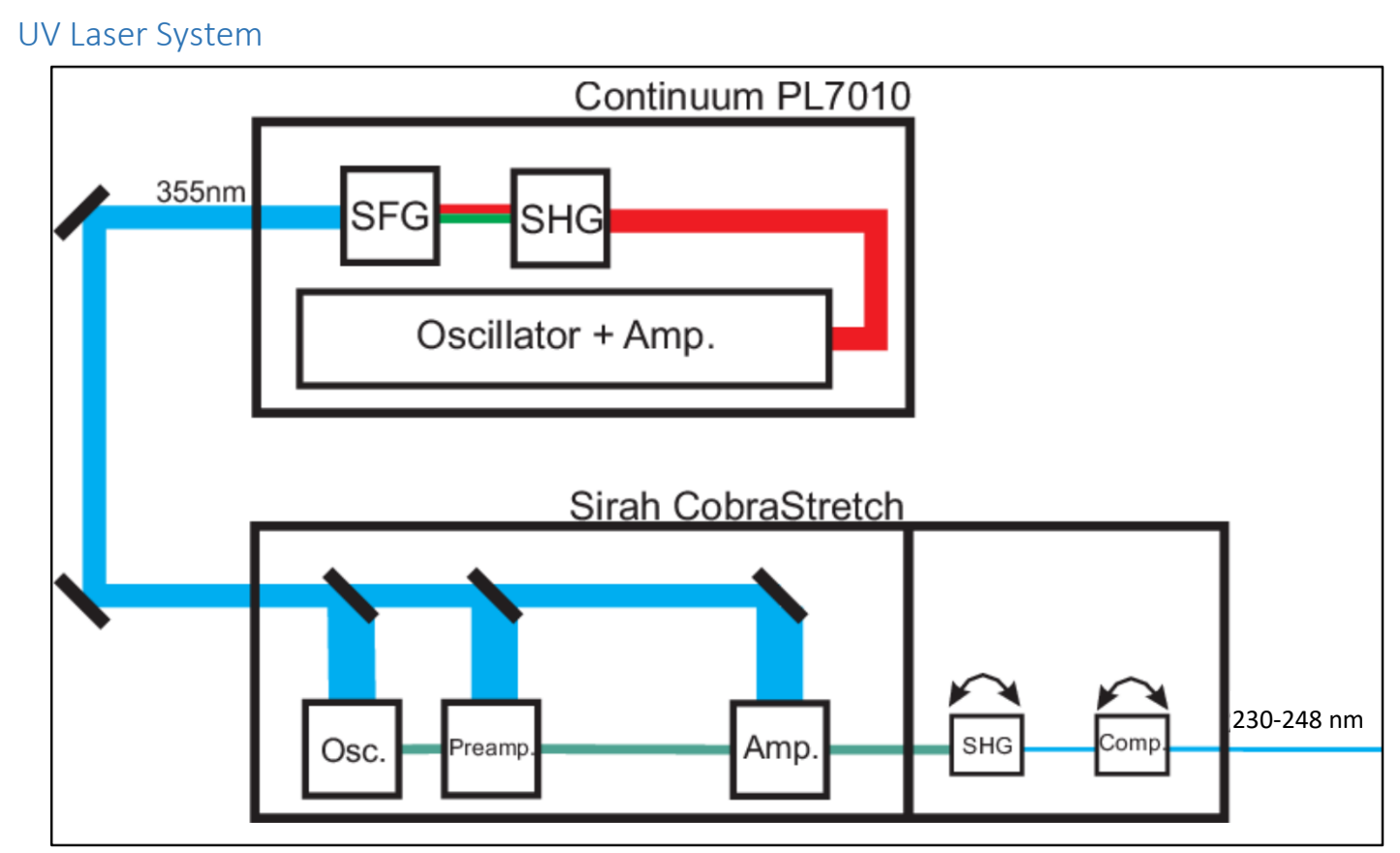

Figure 3.16: UV laser system consisting of a dye laser setup (Sirah Cobra Strech) pumped by a solid state Nd:YAG system (Continuum PL7010).

For the detection of vibrationally excited NO and CO, pulses of UV light in the range of 230-248 nm were produced with the setup depicted Figure 3.16. The third harmonic of a pulsed Nd:YAG laser (355 nm, typically 200 mJ/pulse) was employed to pump a dye laser (Sirah CobraStretch, $0.1 \mathrm{~cm}^{-1}$ ) operated with different coumarin dyes (NO: C480/503 (95:5), CO: C460) providing light in the range of 
460-496 nm. The CobraStretch unit consists of three stages: an oscillator stage where the output wavelength is set and two amplification stages (preamplifier + amplification). Tuning of the laser is achieved by changing the angle of a highly reflective mirror relative to the holographic grating (2400 lines $/ \mathrm{mm}$ ) which selects a narrow part $(110 \mathrm{MHz})$ from the broad fluorescence spectrum of the dye. A BBO ( $\beta$-barium borate, Sirah SHG 215) is employed to produce a frequency-doubled UV output ( $\sim 1 \mathrm{~mJ} /$ pulse). To achieve the optimum output, the angle of the BBO is adjusted accordingly to a previously acquired tuning curve when scanning the wavelength.

\section{IR Laser System}

Beside detection, the preparation of molecules in a desired vibrational state plays an important role in the presented experiments. For both investigated molecules, $\mathrm{NO}$ and $\mathrm{CO}$, this was accomplished by overtone pumping with an intense IR pulse of Fourier-Transform limited bandwidth. To meet all requirements, high intensity and limited bandwidth of IR photons (with tunable wavelength), a rather elaborate laser system was employed. The basic setup is depicted in Figure 3.17. A ring dye laser system (Sirah Matisse DR) operated with a DCM/EPG-EG mixture dye mixture was pumped by a continuous wave (cw) Nd:YLF laser (Coherent Verdi V10) producing light in the range of $660-690 \mathrm{~nm}$ ( $\sim 400 \mathrm{~mW}$ ). Due to the employment of a cw pump laser and a set of wavelength selecting elements (a birefringent filter and two etalons) in the ring resonator, single mode operation with a bandwidth of $20 \mathrm{MHz}$ is achieved. The output is used for pulsed amplification in a five stage amplifier (Sirah PulsAmp 5X) pumped by the second harmonic $(532 \mathrm{~nm})$ of an injection seeded solid state laser (Spectra Physics Quanta Ray Pro 230). The fundamental (1064 nm) of this laser is applied together with the PulsAmp output in the final stages of the setup to produce and amplify IR photons via two nonlinear processes: Difference frequency mixing (DFM) in combination with optical parametric amplification (OPA) yields IR photons from the idler with the wavelength of $2.7 \mu \mathrm{m}$ (overtone excitation of $\mathrm{NO}(v=0 \rightarrow 2)$ ) or respectively $2.3 \mu \mathrm{m}$ (overtone excitation of $\mathrm{CO}(v=0 \rightarrow 2)$ ).

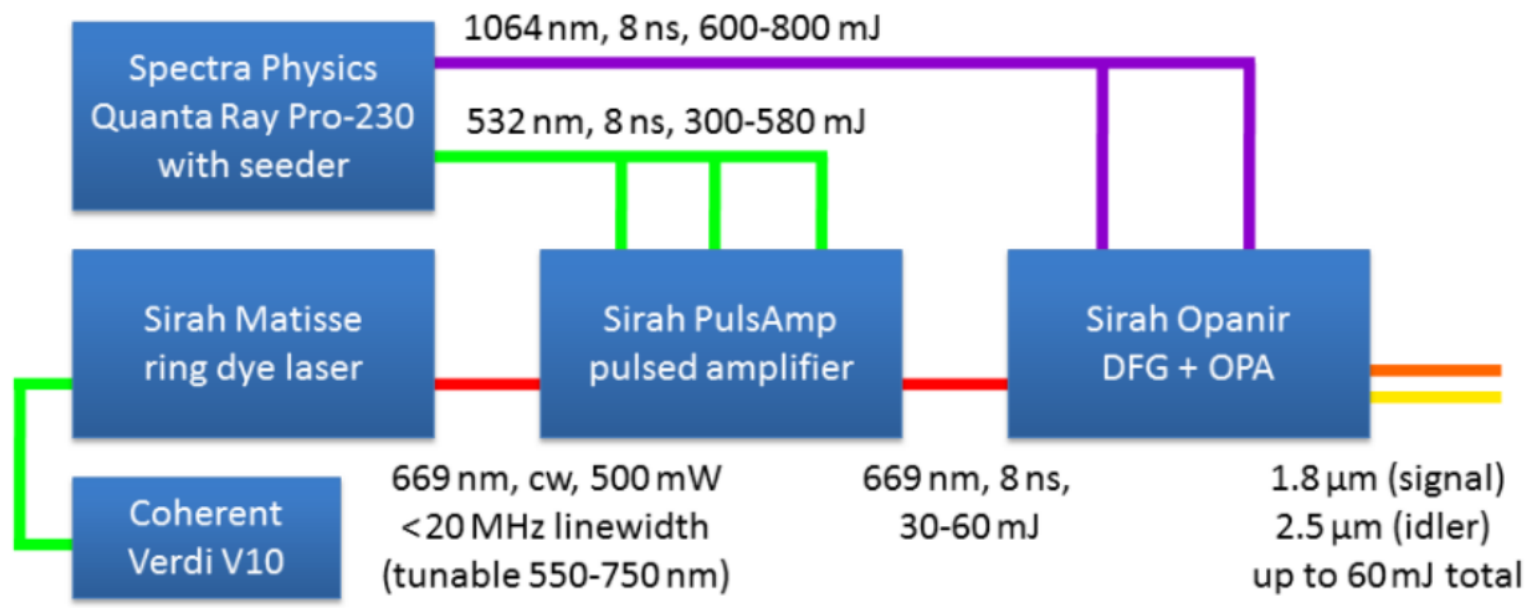

$532 \mathrm{~nm}, \mathrm{cW}, 5 \mathrm{~W}$

Figure 3.17: Setup of the IR source: A ring dye laser (Sirah Matisse) pumped by a (cw) Nd:YLF laser (Coherent Verdi V10) provides visible photons with low spectral width. The cw light is amplified by a 5 stage pulsed amplifier (Sirah PulsAmp) which is pumped by the second harmonic of an injection-seeded Nd:YAG laser (Spectra Physics Quanta Ray Pro-230). With the fundamental of this laser, the amplifier output is converted into IR photons via difference frequency mixing (DFM) and optical parametric amplification (OPA) yielding signal and idler wave. In this work, only the idler was used for molecular tagging. 


\subsubsection{Beamer I: Wedge Sample Preparation and Position/Thickness Assignment}

The biggest part of the thin film samples grown in the molecular beam scattering apparatus were grown in a different manner than those described in the previous characterization section. Instead of subsequent preparation and scattering from a number of homogeneous films, a Ag wedge was grown on the $\mathrm{Au}(111)$ crystal. This was accomplished by employing a movable mask (see Figure 3.6) blocking the beam of silver atoms from the evaporator. This mask, consisting of a razor-like edge is connected with a stepper motor with full control over the mask position and speed to set any desired thickness gradient. This experimental technique which has been previously used successfully in the investigation of thin films [83,84], provides a few advantages over a homogeneous surface: With only a single preparation step, a number of film thicknesses can be prepared; scanning along the wedge is achieved by changing the crystal position with the manipulator. Because the mask blocks a defined part pf the crystal, a clean $\mathrm{Au}(111)$ surface is left for reference measurements. However, the method is limited by the size of the crystal, a cylinder with about $10 \mathrm{~mm}$ diameter. Ignoring the clean Au part and the edges of the crystal where an intensity drop of the scattered molecule signal can be observed, the wedge is grown along $6.5 \mathrm{~mm}$ of the $\mathrm{Au}(111)$ crystal available for scattering experiments. With a fixed size of the IR-tagged molecular beam $(0.5 \mathrm{~mm})$, the choice of a higher thickness gradient is therefore associated with a broader range of thicknesses at each measurement as will be outlined at the end of this section.

After preparation of a wedge, an additional calibration is needed because for the scattering experiments, the film thickness is defined by the manipulator position. This paragraph describes how the surface with a wedge structure is characterized:

As described in a previous section, during deposition almost ideal homogeneity is achieved along the $X$ axis (see Figure 3.12, section 3.1.3). The wedge grown along the $Z$ axis is characterized by taking AES scans along the center of the $X$ axis. The thickness was determined from the $\mathrm{Ag} / \mathrm{Au}$ Intensity ratio and can be plotted against the Auger $Z$ position. As an example, Figure 3.18 shows this graph for a 0-2.4 ML wedge. As desired, a clean $\mathrm{Au}(111)$ part $(Z=93-94.5)$ and a wedge $(Z>=95)$ with almost perfect linear thickness gradient could be grown. The linear trend is important for the thickness determination when values above $4 \mathrm{ML}$ are grown. In this case, the Auger based method cannot be applied; usually the part which can be determined is then extrapolated in a linear manner. The thickness determined close to the right edge $(Z=102)$ is crosschecked with the thickness determined by the QCM showing good agreement.

Finally, the distance between the Auger Spectrometer and the molecular beam needs to be determined. With this offset, we can assign every position during a molecular beam scattering the corresponding thickness. That value is obtained by comparing the Auger Sample current and the scattering signal from a clean $\mathrm{Au}(111)$ crystal. Both patterns match very well when the correct offset is applied (see Figure 3.19) showing a common "flat top" part of the crystal. Additionally, this calibration measurement of the "flat top" REMPI signal is used in the scattering experiments to determine which parts of the crystal can be included in the analysis and which are affected by the crystal edge.

The error of the thickness assignment is mainly influencesd by the thickness gradient along the $Z$ axis. It consists of two aspects: on the one hand, when scanning the surface, the position is set in steps of $0.5 \mathrm{~mm}$, therefore it is reasonable to assume a positioning error of $0.25 \mathrm{~mm}$ in both directions. For the plotted gradient of $0.30 \mathrm{ML} / \mathrm{mm}$ shown in Figure 3.18 that means an error of $<0.1 \mathrm{ML}$.

Since the molecular beam has a finite size, not a single film thickess will be probed. In the most experiments, the interesting measure is the diameter of the IR excited part of the molecular beam. 
From our measurements, we measure a size of $0.5 \mathrm{~mm}$ which would lead to an even bigger error than estimated from the positioning error. From the comparison of high gradient (0- 10 ML wedge) and low gradient wedge (0- 4 ML) wedge, size of the laser focus is an upper limit since we see usually very good agreement. Therefore it seems that the error due to the beam width can be neglected.

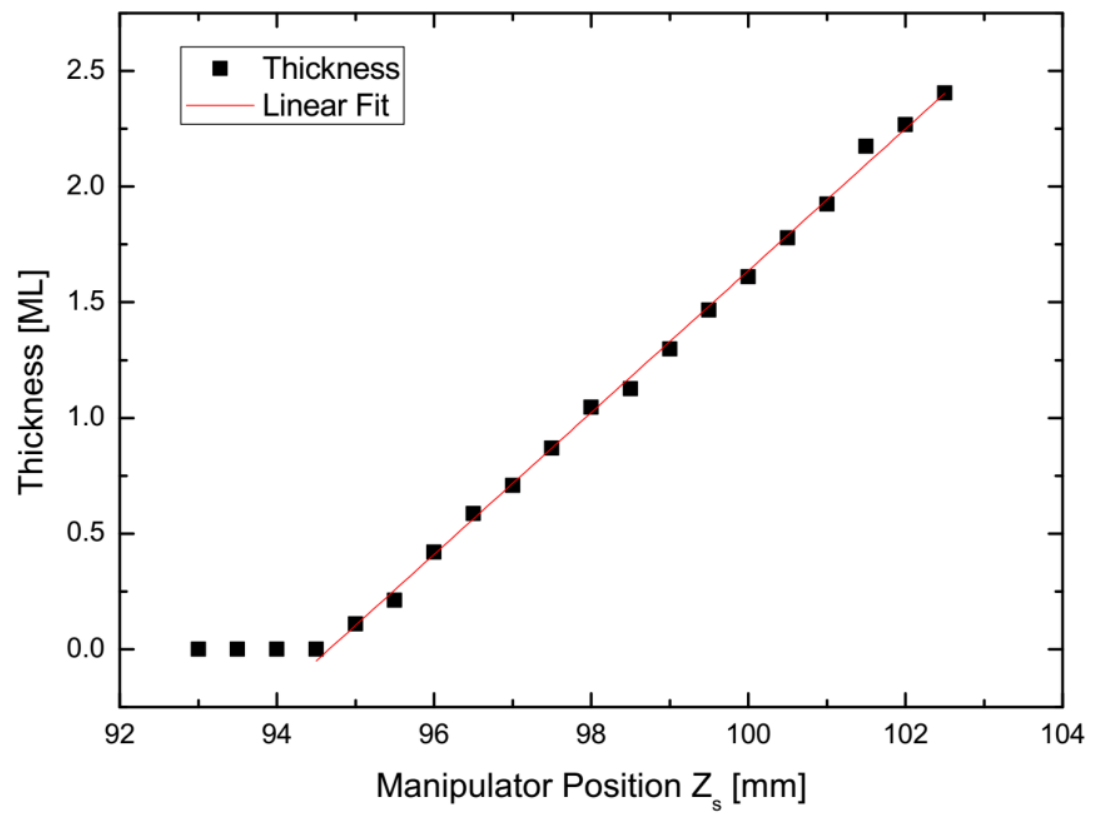

Figure 3.18: Thickness measurement along a 0-2.4 ML wedge, plotted against the manipulator position during the AES measurement. The thickness gradient is following an almost perfect linear increase.

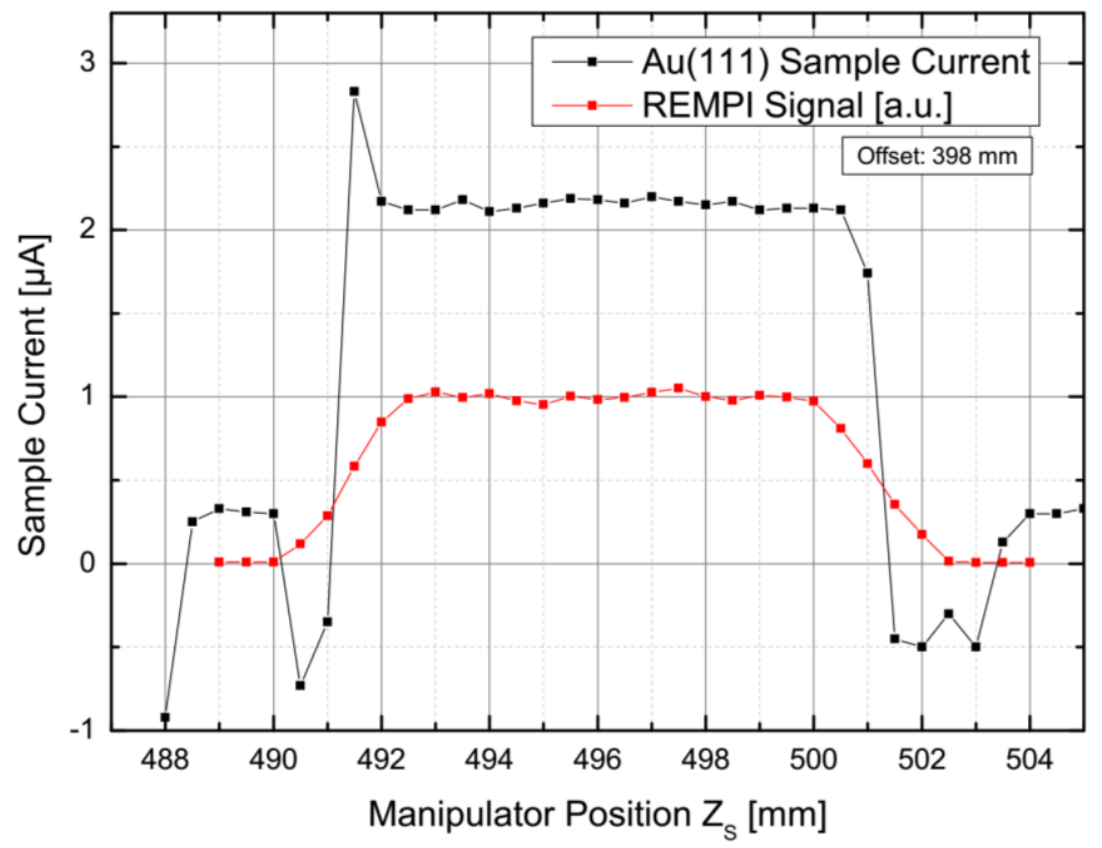

Figure 3.19: Determining the offset between Auger device and molecular beam: Comparison between sample current and REMPI signal of scattered $\mathrm{NO}(v=2 \rightarrow 2)$ along a clean Au(111) crystal. The $x$ axis of the plot shows the $Z$ axis of the crystal in the units during scattering experiments. The Auger sample current was measured with a $3 \mathrm{kV}$ electron beam and maps out the crystal. Due to the slight tilt, the edges pointing towards the electron beam $(Z=491.5)$ and pointing away $(X=501)$ show a slightly different behavior. The NO(v=2 $\rightarrow 2)$ REMPI signal is plotted after correction of the ion collection efficiency and normalization. 


\subsubsection{Experimental Details for NO/CO translational energy transfer}

The first part of the experiments focused on the influence of thickness of a thin silver film for the translational energy transfer of a scattered molecule. This paragraphs describes the details during the TOF measurements after the surface has been prepared and characterized (see 3.2.3). The experimental configuration mainly used in the experiments are shown in Figure 3.20.

(a)

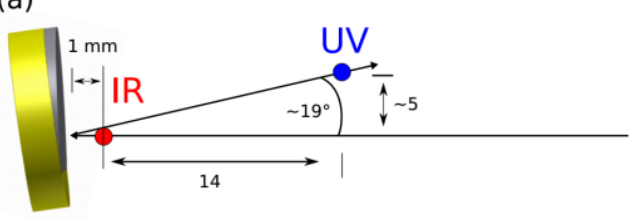

(b)

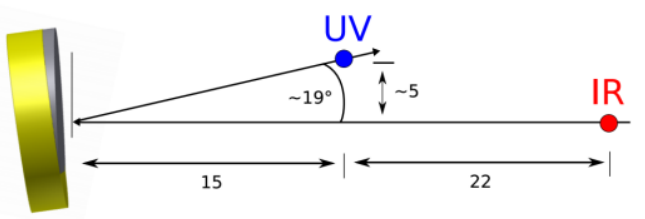

(c)

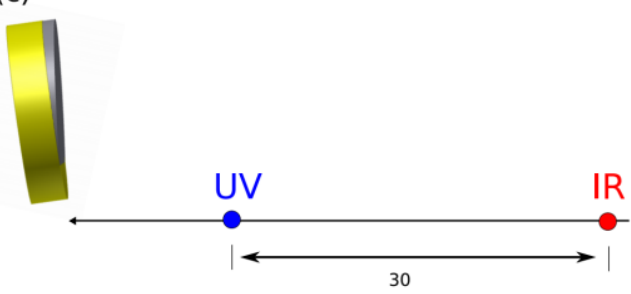

Figure 3.20: Experimental configurations: positions of the REMPI (blue) and IR (red) laser beams relative to the surface and the travelling direction of the incoming molecular beam (black arrow) which is intersected by the laser beams perpendicularly.

State-specific detection: For the case of NO, most experiment were performed with a $7 \% \mathrm{NO} / \mathrm{H}_{2}$ gas mixture (incidence energy of $0.59 \mathrm{eV}$ ). After expansion from the piezoelectric nozzle, the molecular beam is hitting the surface with an incidence angle of $\theta_{i} \approx 7^{\circ}$ with respect to the surface normal. All TOF experiments with NO have been performed with the setup (a) (Figure 3.20, top panel). The IR laser is set at $1 \mathrm{~mm}$ distance to the surface, intersecting the molecular beam. The focused UV laser is displaced by about $5 \mathrm{~mm}$ perpendicular to the molecular beam at a surface distance of $15 \mathrm{~mm}$, corresponding to a scattering angle of $19^{\circ}$ toward the incident beam. The detection at this angle avoids ionization of $\mathrm{NO}$ in the incident beam. With this setup, TOF distributions of $\mathrm{NO}(v=2 \rightarrow 2)$ and $\mathrm{NO}(v=0 \rightarrow 0)$ can be acquired using an established IR-UV "vibrational tagging" technique. For $\mathrm{NO}(v=2 \rightarrow 2,1)$ scattering, the rotationally cold incident $\mathrm{NO}(v=0, J=0.5)$ beam is pumped to $\mathrm{NO}(v=2, J=1.5)$ close to the surface before the collision. NO can be detected in the vibrational states $v=2$ and $v=1$ very efficiently and with rotational resolution via a (1+1) REMPI scheme using the $0-2$ and 0-1 band of the $A^{2} \Sigma^{+} \leftarrow X^{2} \Pi$ transition. The UV laser is set to the desired wavelength to detect the scattered $\mathrm{NO}(v=2 \rightarrow 2,1)$ molecules after $16 \mathrm{~mm}$ flight length from surface. Since both lasers are controlled by delay generators, the temporal delay of the UV laser with respect to the IR laser can be scanned to measure TOF spectra. With the knowledge of the geometries and the time delays, translational energy of the scattered molecules can be determined. A small background signal of $\mathrm{NO}(v=1)$ can be detected resulting from the vibrationally elastic scattering of thermally populated molecules in the incident beam or from collision-induced $\mathrm{NO}(v=0 \rightarrow 1)$ vibrational excitation. A scan 
with the IR blocked was measured for a background correction. The relaxation channel $\mathrm{NO}(v=2 \rightarrow 0)$ cannot be detected due to a strong background of $\mathrm{NO}(v=0)$ molecules in the incident beam. In a similar manner, TOF distributions of $\mathrm{NO}(v=0 \rightarrow 0)$ scattering is performed. For that case, the IR laser is set to a wavelength which is not available in the incident beam but in the scattered molecules. In contrast to the previously described case, the molecules are tagged after collision with the surface in $\mathrm{NO}(v=0 \rightarrow 0)$ scattering.

Generally, the above described experimental details for NO scattering are the same as for TOF experiments with $\mathrm{CO}$. Slight changes are noted in the following paragraph: A gas mixture of $6 \% \mathrm{CO} / \mathrm{H}_{2}$ with $0.64 \mathrm{eV}$ was used for most of the $\mathrm{CO}(v=2 \rightarrow 2,1)$ experiments. Some of the measurements were performed with the setup of (b) (Figure 3.20, central panel) with the intention to avoid the possibility of a tagging of molecules which have already collided with the surface. However, it was found that the experimental setup does not influence the shape of the TOF distributions (see Figure 3.21), although it was expected that the additional travel path could lead to broadening in time.

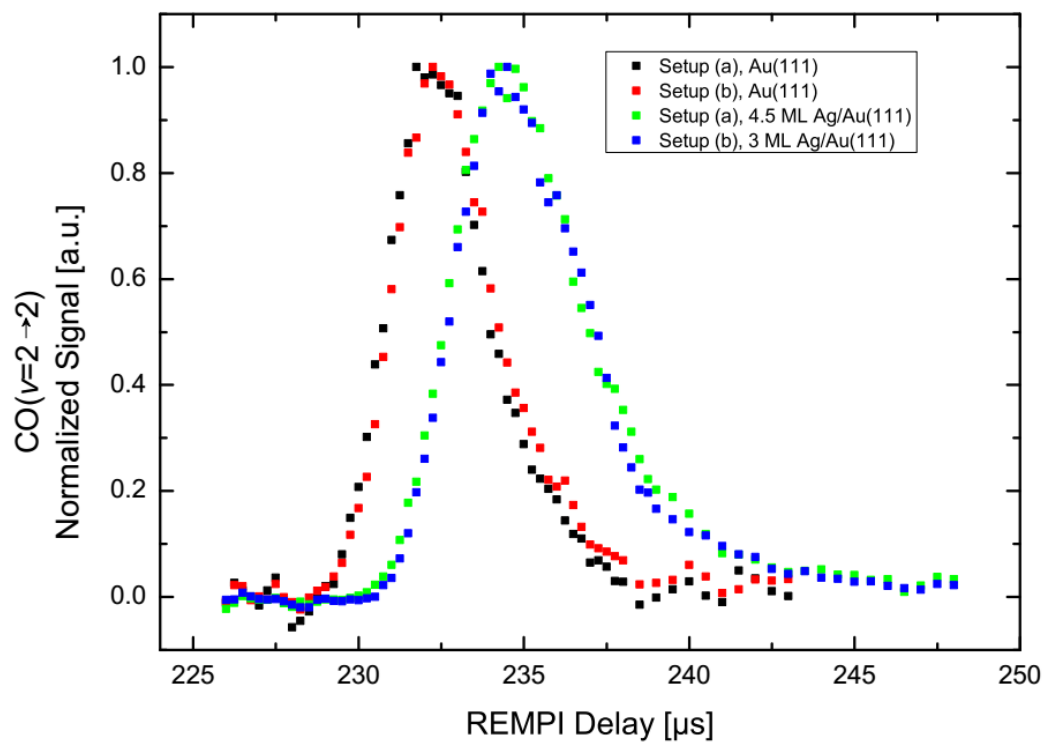

Figure 3.21: No influence of the IR tagging position: TOF shapes of $\mathrm{CO}(v=2 \rightarrow 2)$ scattering from $A u(111)$ and $3 \mathrm{ML} A g / A u$ at different IR tagging positions. Setup (a) and (b) are referring to the experimental setup depicted in Figure 3.20. A common gas mixture $\left(6 \% \mathrm{CO} / \mathrm{H}_{2}, 0.64 \mathrm{eV}\right)$ was used.

Scattered CO molecules in $v=2$ and $v=1$ were detected in a (2+1) REMPI scheme using the 1-2 and 11 bands of the $\mathrm{B}^{1} \Sigma^{+} \leftarrow \mathrm{X}^{1} \Sigma^{+}$transition. In comparison to NO, the ionization of $\mathrm{CO}$ is less efficient, leading to a lower signal-to-noise ratio. To account for non-resonant ionization, a scan with the IR blocked was measured for background correction.

The vibrational tagging approach can also be applied to measure the incident energy of a molecular beam. In this case, the experimental setup (c) (Figure 3.20) is used and the crystal is moved up to avoid any scattering. After IR excitation, the incoming beam is traveling $30 \mathrm{~mm}$ before the UV laser leads to ionization via REMPI. The timings of the lasers are controlled by delay-generators. Combining the information about the distances and the flight time allows to calculate the translational energy distribution of the incoming beam.

Approach to detect rotationally near-elastic scattering: During the scattering event of $\mathrm{NO}$ and $\mathrm{CO}$ and from metal surfaces, a part of the incident translational energy is converted into rotational energy of the scattered molecules $[85,86]$. As a consequence, the final kinetic energy of a scattered molecule is dependent on the rotational state. Therefore, the translational energy for the near-elastic scattering case is typically used as a comparison measure which is obtained in the following way: TOF 
distributions from a number of rotational states are measured. For every state, the mean kinetic energy $\left\langle E_{f}\right\rangle$ is calculated and plotted against the corresponding rotational energy; the final translational energy is obtained via extrapolation to $J=0$. In this work however, that value is obtained by focusing on $\mathrm{NO}(v=2 \rightarrow 2)$ in a single rotational state $J=5.5$ which is a good approximation [87] but might underestimate the elastic case by $5 \%$. In an analogue manner, $\mathrm{NO}(v=0 \rightarrow 0)$ scattering was as well performed in a low rotational state $(J=6.5)$.

A similar strategy was chosen for CO: Note that in contrast to the detection of NO, the employed REMPI scheme for $\mathrm{CO}$ does not provide rotational resolution which makes single rotational state detection impossible. A comparison with NO is still possible if the overlapping detected states only include low rotational states which might serve as a good approximation for the near-elastic case as in NO. TOF shapes were obtained at the peak of the respective spectrum $(230.19 \mathrm{~nm}$ for $\mathrm{CO}(v=2 \rightarrow 1)$ and $235.94 \mathrm{~nm}$ for $\mathrm{CO}(v=2 \rightarrow 2))$. From a comparison to a spectral simulation, it is estimated that in both branches, a number of 1-4 rotational lines of low rotational states $\left(J_{\max }=7\right)$ is detected simultaneously. A dataset for $\mathrm{CO}(v=0 \rightarrow 0)$ scattering from bulk $\mathrm{Au}(111)$ and $\mathrm{Ag}(111)$ with similar incidence energy shows that rotational states up to $J=8$ exhibit the same TOF distribution, suggesting that the requirements described above are met, in accordance to state-to-state measurements on $\mathrm{Ag}(111)$ [86].

With a kinetic energy of $\mathrm{CO}\left(\mathrm{E}_{\mathrm{i}}=0.64 \mathrm{eV}\right)$ which is approximately the same as for the NO mixture $\left(E_{i}=0.59 \mathrm{eV}\right)$, a direct comparison of both molecules is possible. Under the described measurement conditions, the final translational energy of the near rotationally-elastic case can be obtained and compared with the literature.

\subsubsection{Experimental Details for REMPI Signal Intensity Experiments}

The TOF studies of the first part pointed at the translational energy transfer of a molecule scattered from a thin film. In the second part, the main interest is on the vibrational energy transfer of $\mathrm{NO}(v=2)$ and $\mathrm{CO}(v=2)$ when scattered off a surface coated with silver. For those experiments, the REMPI signal intensities of the $v=2 \rightarrow 2$ and $v=2 \rightarrow 1$ scattering channel were detected for different film thicknesses. The signal strength from $\mathrm{Au}(111)$ was chosen as a common reference. From the thickness dependency of the REMPI signals, a relaxation probability of $\mathrm{NO}(v=2)$ and $\mathrm{CO}(v=2)$ can be determined.

Signal intensity measurements: The REMPI signals of scattered $\mathrm{NO}(v=2)$ are obtained in two different approaches which yield the same result. In first case, the signal intensity of the desired vibrational state is measured by focusing on a single rotational state. It has to be avoided to choose a $J$ state which could be present in the scattered molecules due to IR tagging of rotationally excited $\mathrm{NO}(v=0)$ molecules after the surface collision. After preparation and characterization of the surface, molecular beam scattering experiments are carried out with the geometrical configuration depicted in setup (a) (Figure 3.20, top panel). First, TOF distributions are obtained by scanning along the crystal by changing its position relative to the incident molecular beam. The main goal of this measurement is to find the peak timings of the TOF distributions. Second, the obtained peak timing can then be set when detecting the signal intensity for different sample positions. Both approaches, scanning the surface in $0.5 \mathrm{~mm}$ steps or two subsequent measurements in $1.0 \mathrm{~mm}$ along the surface yield the same result within the uncertainties of about $5 \%$. In a typical experiment, the REMPI signal for a defined $J$ - and $v$-state is acquired for 10-20 s, laser power of the REMPI (UV) laser is about $1.0 \mathrm{~mJ} /$ pulse. To minimize any influence of the IR laser power, it was measured before and after every scan to ensure that it is always above the saturation threshold $\left(P_{\mathrm{IR}}>5.5 \mathrm{~mJ} /\right.$ pulse, see Figure 3.22$)$. 


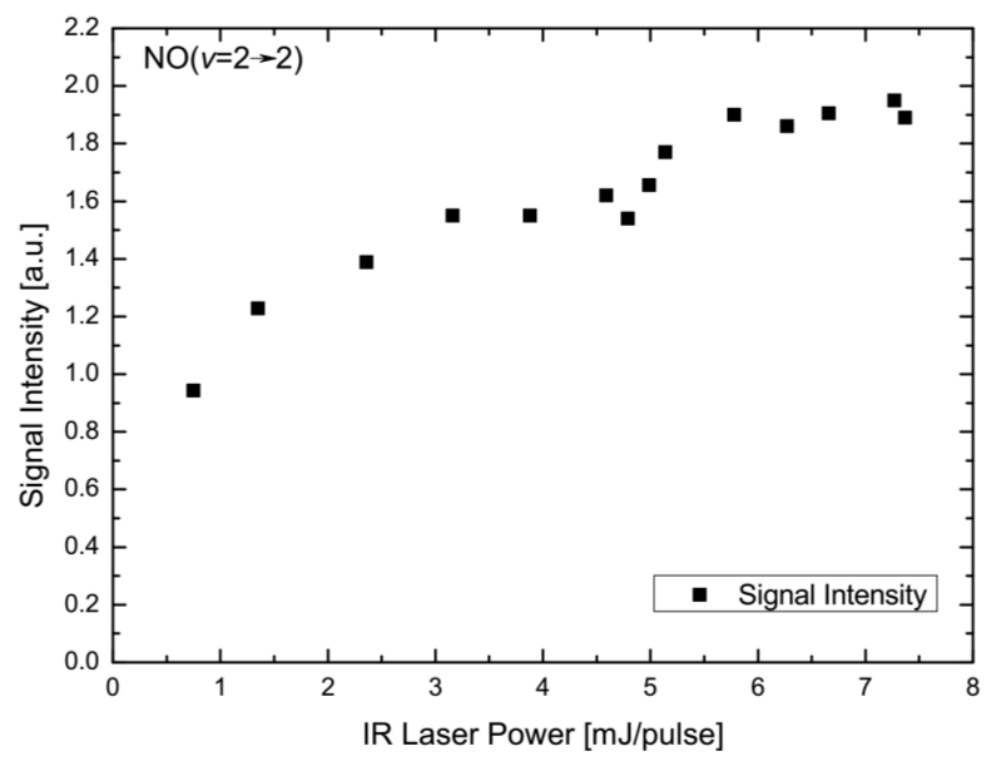

Figure 3.22: $N O(v=2 \rightarrow 2)$ REMPI signal dependency on IR laser power. For laser powers above $5.5 \mathrm{~mJ}$, the REMPI signal is constant and any correction of the REMPI signal can be neglected. The laser power is measured after passing through the apparatus at the entrance window for the UV laser.

To account for fluctuations in the REMPI (UV) laser, a beam splitter was employed to reflect a fraction of the UV REMPI laser which was monitored as a measure for the pulse power of the incident laser beam (see Figure 3.23). A shot-to-shot laser power correction is possible with this data; however, in the case of REMPI signals from a single rotational state, the influence of fluctuations on the detected REMPI signal is negligible. Finally, the average signal of molecules scattered off positions with clean $\mathrm{Au}(111)$ was used as a common reference and therefore set to the value of 1.0.

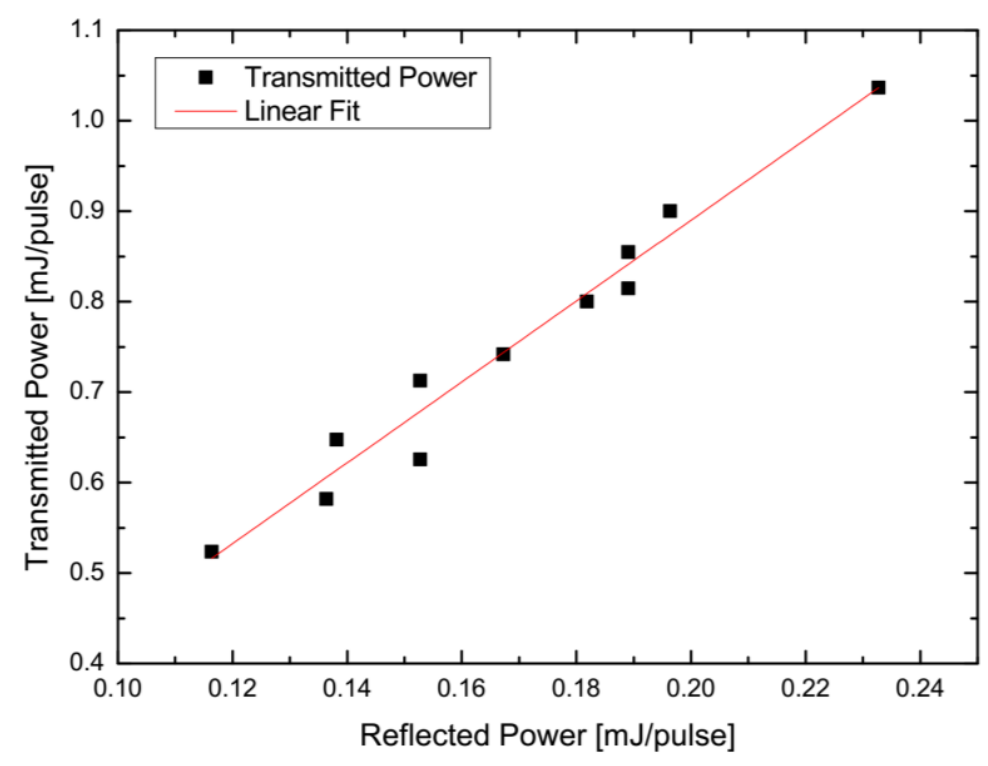

Figure 3.23: A beam splitter is employed to reflect a fraction of the UV laser. The graph shows the relation between transmitted and reflected power at three different wavelengths $(236,242.5,246 \mathrm{~nm})$.

For the second approach, the $\mathrm{NO}(v=2 \rightarrow 2,1)$ REMPI signals were obtained by measuring a full spectrum of the vibrational band. Those experiment were carried out with setup (b) (Figure 3.20, central panel) to avoid the tagging of molecules after scattering from the surface. Surface preparation 
was carried out in a different manner: After sputtering, annealing and cooling to room temperature, the crystal was coated in a way that $50 \%$ of its surface were blocked by the mask (see section 3.2.3). That way, half of the surface is clean $\mathrm{Au}(111)$, the other half consists of a homogeneously coated silver film with defined thickness. Peak timing was determined by measuring a TOF distribution of the rotational state $J=11.5$ which is close to the maximum in the rotational distribution of the scattered molecules. REMPI signals were obtained by scanning the wavelength range $242.5-248 \mathrm{~nm}$ for $\mathrm{NO}(v=2)$, respectively $233.5 \mathrm{~nm}-238.8 \mathrm{~nm}$ for $\mathrm{NO}(v=1)$. A fast scan rate is set which allows the detection of a vibrational band in about $2 \mathrm{~min}$. Scans of $\mathrm{NO}(v=2 \rightarrow 2)$ and $\mathrm{NO}(v=2 \rightarrow 1)$ are carried out on both surfaces, including a background scan for $\mathrm{NO}(v=2 \rightarrow 1)$ with the IR blocked. REMPI and IR laser powers are monitored in the same manner as described previously. At least 5 datasets have been acquired for one film thickness. For the analysis, after baseline-correction and REMPI laser power correction (linearly depending on the UV laser power), the spectra were integrated to get the signal strength; in the case of $\mathrm{NO}(v=2 \rightarrow 1)$ the intensity is additionally corrected by subtracting the background signal. Signal strengths are scaled by employing the $\mathrm{NO}(v=2 \rightarrow 2,1)$ signal from $\mathrm{Au}(111)$ as reference value.

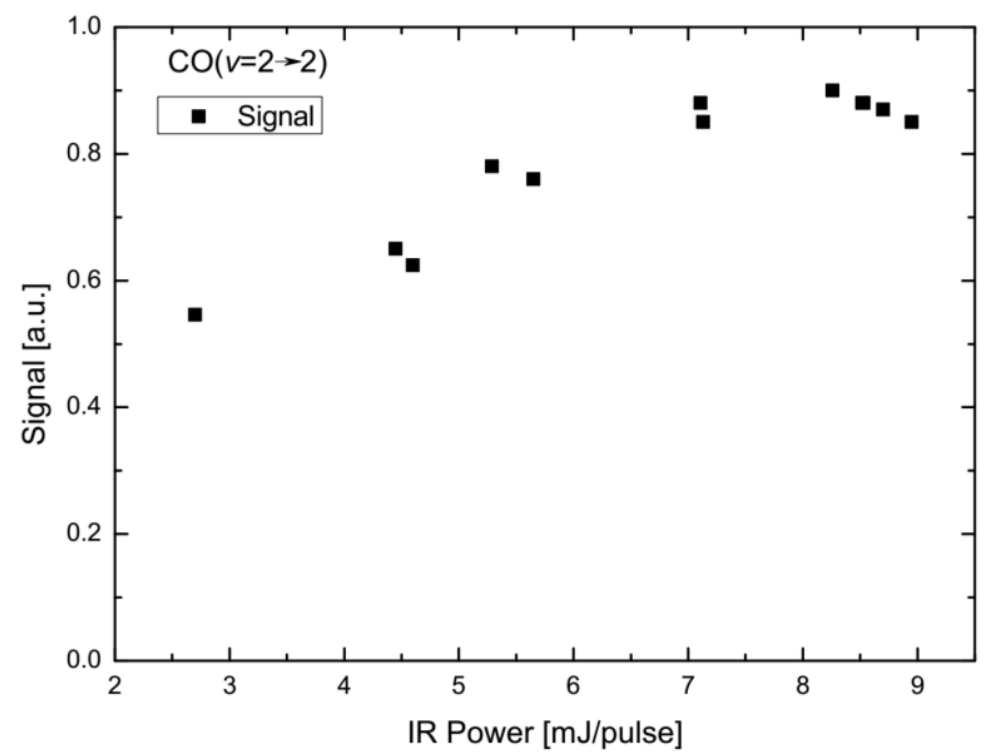

Figure 3.24: Analog to Figure 3.20: $\mathrm{CO}(\mathrm{v}=2 \rightarrow 2)$ REMPI signal dependency on IR laser power. For laser powers above $7 \mathrm{~mJ}$, the REMPI signal is constant. The laser power is measured after passing through the apparatus.

The general procedure described for the detection of REMPI signals for NO is kept when switching the probe target to $\mathrm{CO}$. As has been noted for the TOF experiments, the REMPI signal intensities were measured partially in setup (a) and (b) (Figure 3.20) yielding the same results as seen in Figure 3.28 (right panel). The UV laser was set to the peak of the respective spectrum $(230.19 \mathrm{~nm}$ for $\mathrm{CO}(v=2 \rightarrow 1)$ and $235.94 \mathrm{~nm}$ for $\mathrm{CO}(v=2 \rightarrow 2))$ before determining the peak timing from a TOF measurement. REMPI signals were measured in the same way as described for NO. Again, to minimize its influence on the measurement, the IR laser power was measured before and after every scan to ensure that it is always above the saturation threshold (for CO: $P_{\mathbb{R}}>7 \mathrm{~mJ} /$ pulse, see Figure 3.24). Due to the two photon transition in the first step of the $(2+1)$ REMPI scheme using the $\mathrm{B}^{1} \Sigma^{+} \leftarrow \mathrm{X}{ }^{1} \Sigma^{+}$ transition, the UV laser power and its fluctuations are having a stronger influence during the acquisition of the signal strengths. Instead of using a beam splitter as NO and a potential loss of incident laser power, the UV laser power is monitored at the end of the chamber after passing through the two windows. From the obtained signal, the incident UV pulse power could be determined (see Figure 3.25) and was used to correct laser power fluctuations. 


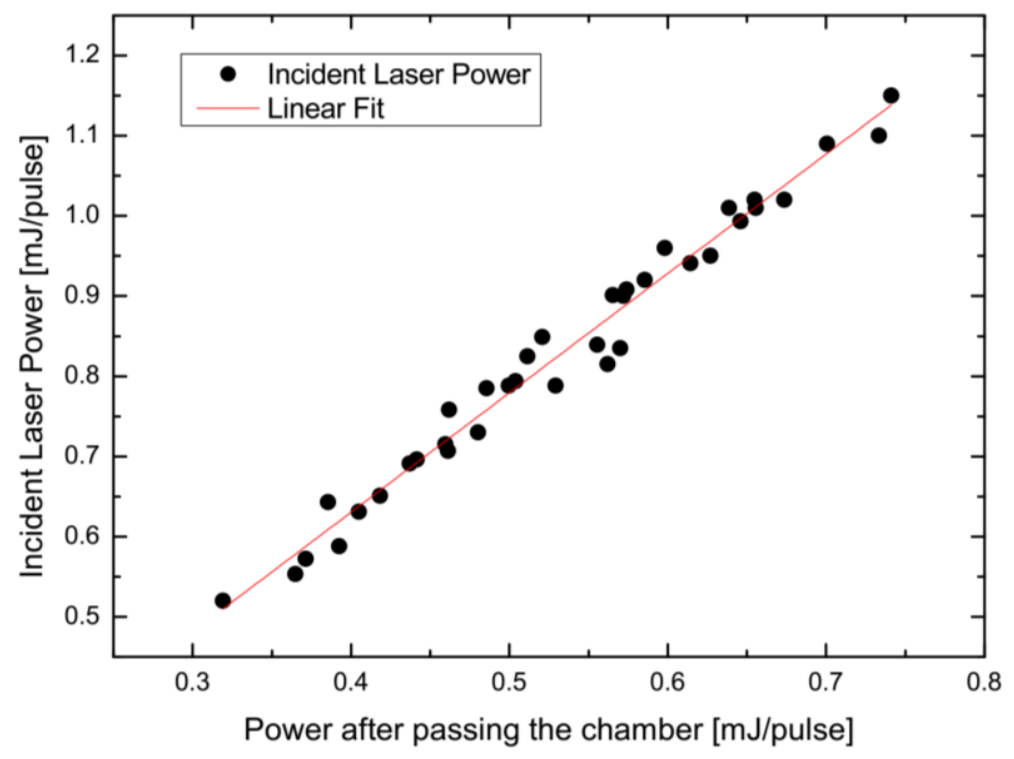

Figure 3.25: Monitor UV laser power during the CO REMPI signal measurements: incident UV laser power vs monitor signal measured after passing the chamber.

Obtaining the "Ion Collection Efficiency": The state-specific REMPI intensities of a molecule scattered off the crystal are acquired using the previously described procedure. For the quantitative comparison of those signals obtained from different manipulator positions, any changes due to the configurations need to be taken into account. The ions which are produced in the REMPI process are following the electric field lines between the repeller and the ion lenses (see Figure 3.15) before being detected at the MCP. The surface with the manipulator is taking part in forming this electric field, so a change of the manipulator position might lead to changes of the efficiency to collect the ions.

As a calibration measurement, the clean and unprepared surface was used as a target. With the configuration setup (a) (Figure 3.20, top panel) the $\mathrm{NO}(v=2 \rightarrow 2)$ signal was obtained. The raw signal (only scaled by a constant) is depicted in the left panel of Figure 3.26. The shape of the crystal $(\varnothing=10 \mathrm{~mm})$ is mirrored in the signal of the scattered molecule. Clearly, a linear signal increase with the change of the manipulator position can be recognized.
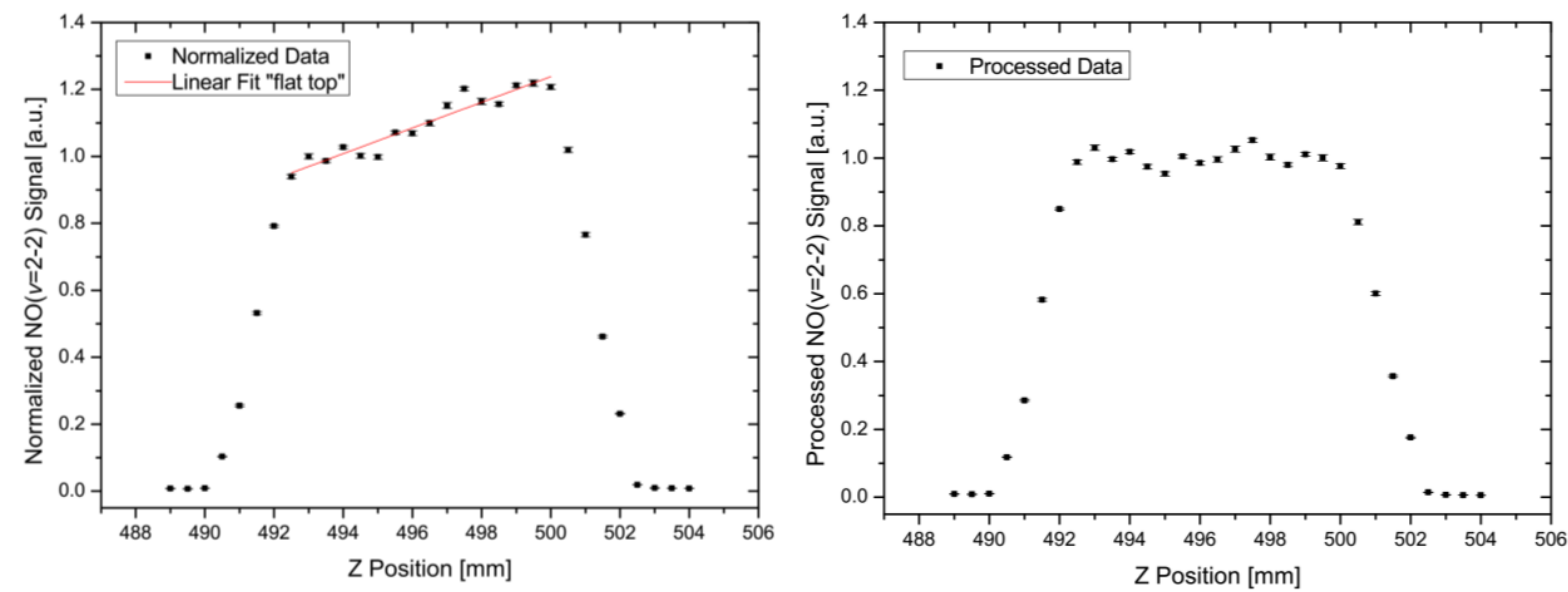

Figure 3.26 Left panel: $\mathrm{NO}(v=2 \rightarrow 2)$ REMPI signal calibration measurement along a clean Au(111) crystal using experimental setup (a) (Figure 3.20, top panel). The signal was normalized that $Z=493 \mathrm{~mm}$ (a position is clean Au(111) on almost all grown wedge structures) represents unity. The "flat top" region is fitted by a linear function. Right panel: After applying a linear correction, a constant signal with manipulator position is obtained. 
This signal increase is interpreted as a change of the ion collection efficiency. Since the $\mathrm{NO}(v=2 \rightarrow 2)$ signal detected as scattered from any part of the (flat) Au crystal is expected to show the same intensity, a linear correction is performed according to the signal increase obtained from the flat part of the signal. When this correction function is applied on the data, a processed signal with a constant value is yielded (Figure 3.26, right panel). Because the measurement technique contains this inherent signal increase when scanning along the crystal, this correction is performed for any measurements on a wedge. In chapter 5 and 6 any signal shown (and referred to as "raw signal") will contain this correction.

A similar test measurement was performed with CO. Those experiments were carried out in two different experimental configurations (a) and (b) (Figure 3.20, top and central panel). When comparing the data obtained for both setups, it was found out that the ion collection efficiency showed a dependency on the experimental setup: Measuring the $\mathrm{CO}(v=2 \rightarrow 2)$ signal along a clean $\mathrm{Au}(111)$ crystal with setup (a), a significant signal increase could be observed (Figure 3.27, left panel). However, when the measurement configuration (b) was chosen, the signal increase is negligible (Figure 3.27, right panel). The same result was obtained when measuring the $\mathrm{CO}(v=2 \rightarrow 1)$ signal. As a consequence, the correction function on the raw data was chosen according to the setup used during the data acquisition.
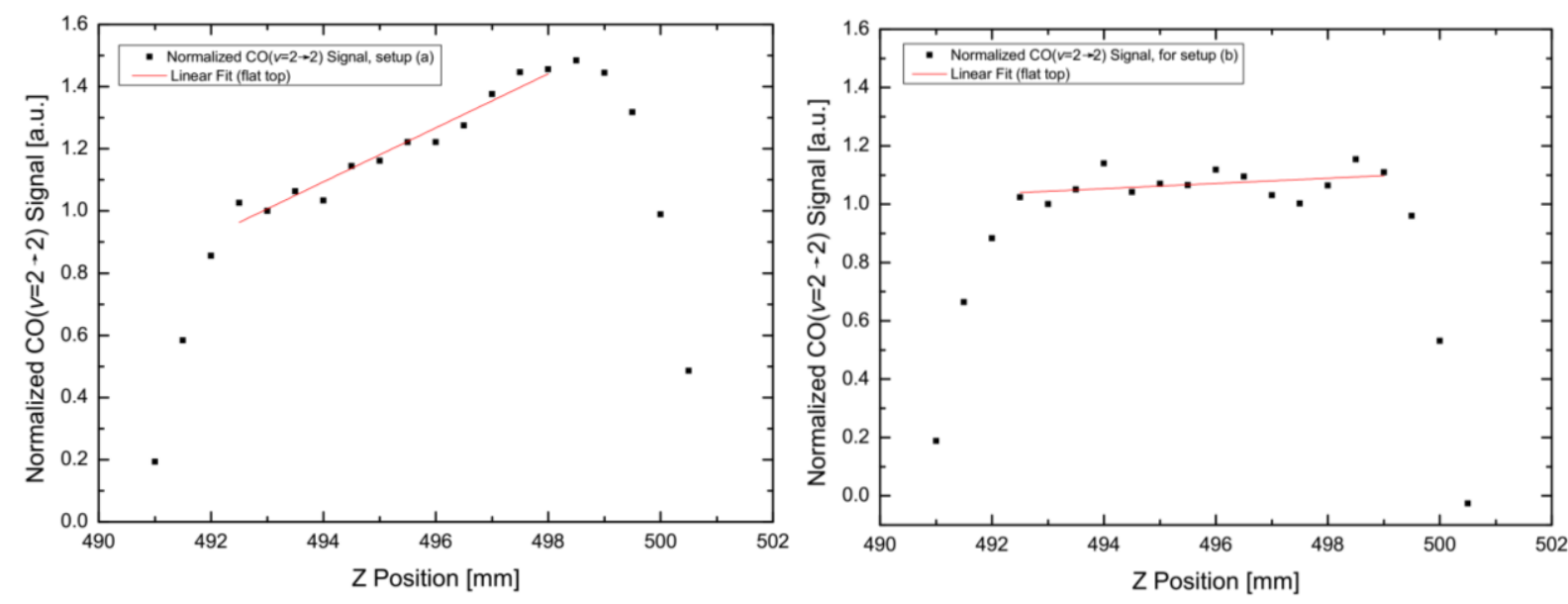

Figure 3.27: Dependency of the ion collection efficiency with experimental setup: The $\mathrm{CO}(v=2 \rightarrow 2)$ signal is measured along the clean $A u(111)$ crystal. With the IR tagging the molecular beam directly in front of the surface, (setup (a)), a significant signal increase is obtained. However, with tagging with at $\sim 36 \mathrm{~mm}$ distance to the surface, no signal increase is observed.
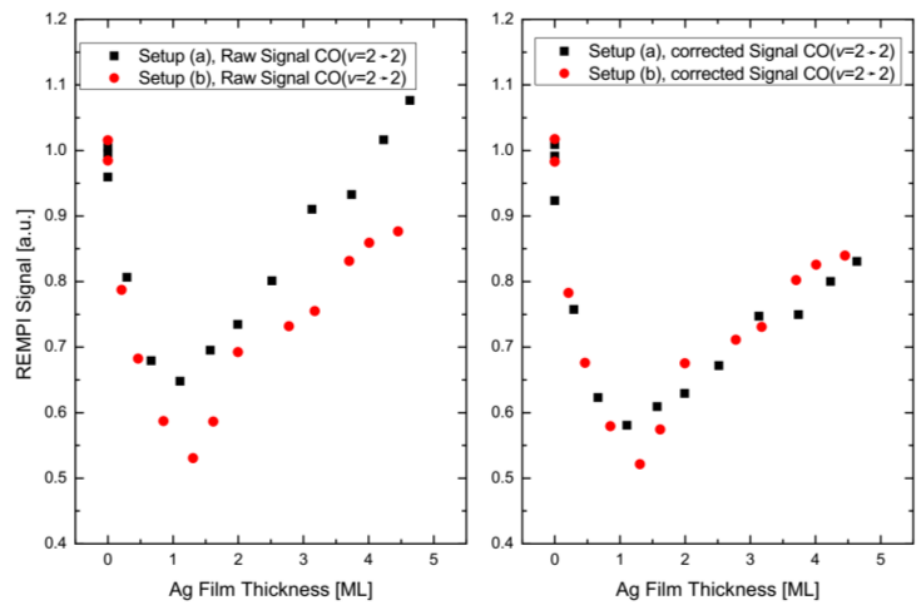

Figure 3.28: Testing the signal calibration depending on the experimental setup. Left: $R a w C O(v=2 \rightarrow 2)$ REMPI signals from 0-6 ML wedge Ag/Au depending on film thickness. The signal from Au(111) was taken as a reference and set to 1. Right: Both signals are corrected by taking into account the position-dependent ion collection efficiency obtained from experimental data. 
This procedure can be tested on two datasets measured on an equal 0-6 ML wedge but with different experimental setup. For both cases, the raw $\mathrm{CO}(v=2 \rightarrow 2)$ signal is plotted against the manipulator $\mathrm{Z}$ position (see Figure 3.28, left panel). Both datasets show the same trend but only reasonable agreement. Taking into account the different ion collection efficiency depending on the experimental setup and applying a correction function which was determined form the previously described scans along a clean $\mathrm{Au}(111)$ crystal leads to a stronger agreement between both datasets (Figure 3.28, right panel). A similar procedure is carried out when measuring the $\mathrm{CO}(v=2 \rightarrow 1)$ signal.

Although consistent measurements can be achieved using the described method, the origin of this signal increase remains obscure. If this effect can be ascribed to a change of the change of the electric field, a signal increase should be visible when measuring the REMPI signal when the chamber is filled with a background gas. With a background pressure of $3.8 \times 10^{-8}$ Torr $\mathrm{CO}$, the REMPI laser was set a wavelength to detect $\mathrm{CO}(v=0)$ and the REMPI signal was measured at different manipulator positions (Figure 3.29). However, no signal increase can be observed.

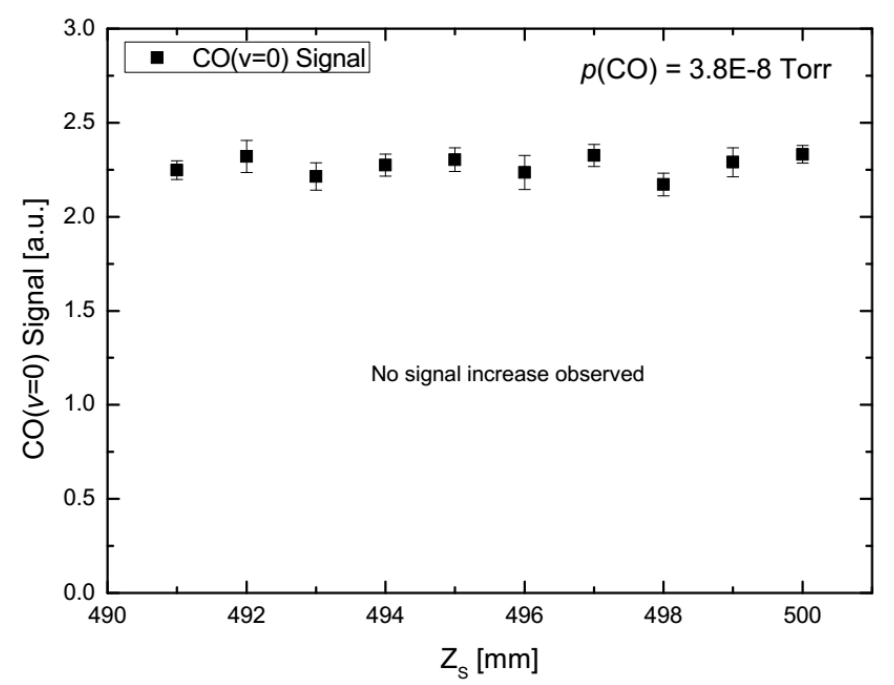

Figure 3.29: Ion collection efficiency measurement. The REMPI signal of $C O(v=0)$ is measured with a background pressure of $3.8 \times 10^{-8}$ Torr CO. No signal increase can be observed.

Why should the tagging position influence the collection efficiency? What is the difference between molecules detect $1 \mathrm{~mm}$ in front of the surface or at a position $>30 \mathrm{~mm}$ distance? A possible difference is that traveling for a longer distance until hitting the surface (setup (b)) might lead to a broadening in time or space. With a difference in the density, the collection efficiency could vary. Those assumptions can be tested: From TOF measurements, the arrival time distribution does not change depending on the tagging position (see Figure 3.21). Furthermore, within the experimental error, the angular distribution does not change with the tagging position.

\subsubsection{Experimental Details for Angular Distribution Measurements}

For NO, angular distributions are measured in the experimental configuration shown in Figure 3.20, (setup (a), top panel). After finding the peak timing for $\mathrm{NO}(v=2 \rightarrow 2,1, J=11.5)$ which is close to the rotational distribution of the scattered molecules, the detection angle is set by moving the REMPI laser perpendicular to the molecular beam in a range of $\sim 10 \mathrm{~mm}$. IR laser power is checked for stability before and after each measurement. For measurements of $\mathrm{NO}(v=2 \rightarrow 1)$, background scans are carried out. 
For $\mathrm{CO}$, a slightly different procedure is chosen. To increase the sensitivity of the measurement, the REMPI is $8 \mathrm{~mm}$ shifted towards the surface in comparison with setup (a) (Figure 3.20, top panel). An additional advantage is that the ion collection efficiency is changing in a gradual manner. The detection angle is set similarly as for NO. However, when scanning different angles, the flight path of the scattered molecules is changing between $\sim 8$ and $\sim 12 \mathrm{~mm}$, therefore the REMPI delay had to be set depending on the measurement position. $\mathrm{CO}(v=2 \rightarrow 2,1)$ is measured at the signal intensity peak of the corresponding vibrational state $(230.19 \mathrm{~nm}$ for $\mathrm{CO}(v=2 \rightarrow 1)$ and $235.94 \mathrm{~nm}$ for $\mathrm{CO}(v=2 \rightarrow 2))$. 


\section{Translational Inelasticity in Molecular Beam Scattering from Ultrathin Films of $\mathrm{Ag} / \mathrm{Au}(111)$}

Since the advent of molecular-beam scattering techniques in combination with UHV technology it was possible to perform first experiments to characterize the energy transfer between a gas molecule and a solid during a collision event. Furthermore, the advances in laser-based techniques allowed to prepare and detect molecules in a state-selective manner. With those tools, influences of the initial translational, rotational and vibrational energy on the collision event could be investigated with high resolution. Comparison between the initially prepared and the scattered molecules gave detailed insights about a) the energy transfer from gas molecule to the solid, b) which channels of the solid are taking part in energy exchange and c) how the total energy of the scattered molecule after the collision event is redistributed within the available degrees of freedom.

This chapter focuses on the translational energy transfer during the scattering process of the molecules from the thin film system $\mathrm{Ag} / \mathrm{Au}(111)$. The approach is to explore energy transfer on an atomic scale on this well-described system whose growth proceeds in a layer-by-layer fashion. Structural and electronic properties have already been subject to a number of studies. To my knowledge, this is the first state-to-state scattering study about the film thickness effect on translational energy transfer. It was the motivation to address the following two questions: (1) How does the energy transfer change with film thickness? (2) For which film thickness can we observe a behavior equal to the bulk crystal of the deposit?

First, vibrationally elastic NO scattering is investigated. This is conceptually the simplest case, seems to be well understood and can therefore be compared to already existing data obtained from bulk surface scattering. Because of its simplicity this method is ideal to obtain additional information regarding surface quality.

In a second step, it will be focused on the juxtaposition of vibrationally elastic and inelastic scattering. For NO and $\mathrm{CO}$, both scattering channels $v=2 \rightarrow 2,1$ were examined from thin films. Beside the thickness-dependent scattering behavior, the increase of translational energy after relaxation will be compared for both investigated molecules. 


\subsection{Results}

\subsubsection{Vibrational Elastic Scattering of $\mathrm{NO}(v=2 \rightarrow 2)$ and $\mathrm{NO}(v=0 \rightarrow 0)$}
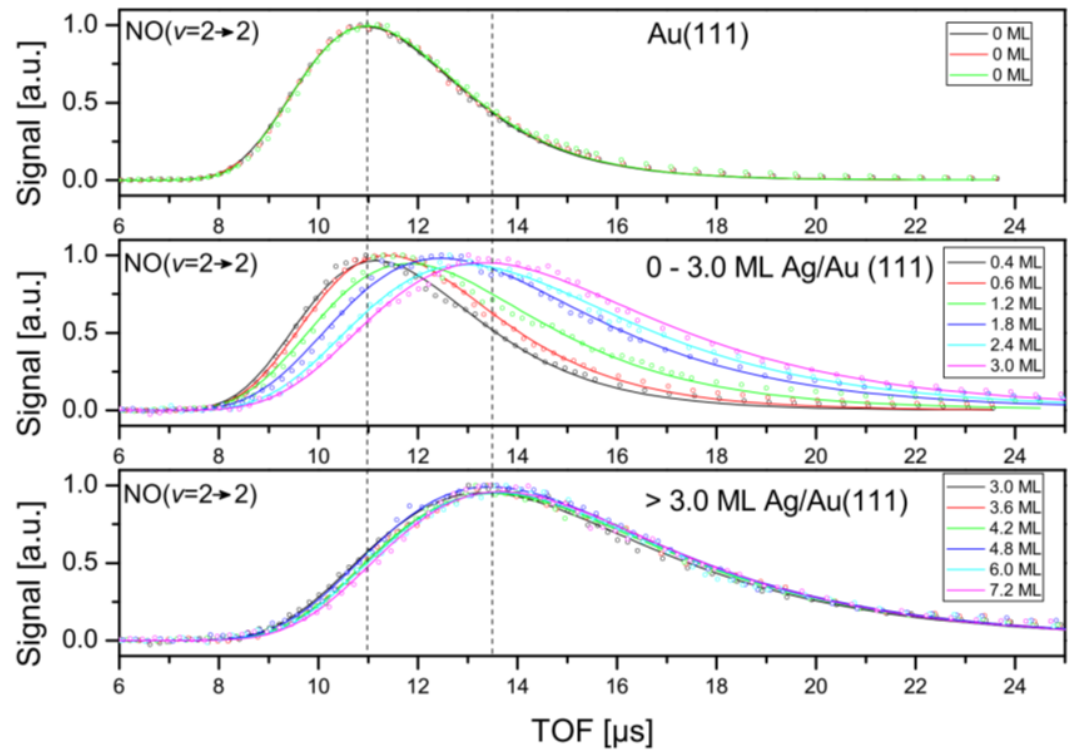

Figure 4.1: State-to-state time-of-flight spectra of $\mathrm{NO}(v=2 \rightarrow 2, J=5.5)$ scattered off a thin film with thickness gradient of $0-7.2 \mathrm{ML} \mathrm{Ag/Au(111).} \mathrm{Depicted} \mathrm{is} \mathrm{the} \mathrm{experimental} \mathrm{data} \mathrm{(empty} \mathrm{circles)} \mathrm{and} \mathrm{corresponding} \mathrm{fit} \mathrm{function} \mathrm{(solid} \mathrm{lines).} \mathrm{Data} \mathrm{sets}$ are separated into three groups depending on the chosen surface characteristic: clean Au(111) (top panel); $\mathrm{Ag} / \mathrm{Au}(111)$ film with thickness of $0-3 \mathrm{ML}$ (central panel); $\mathrm{Ag} / \mathrm{Au}(111)$ film with thickness of $>3 \mathrm{ML}$ (bottom panel). Peak timing of both limiting cases (clean Au and bulk limit of $\mathrm{Ag} / \mathrm{Au}$ ) are marked by two dashed vertical lines.

First, the case of vibrationally-elastic scattering of $\mathrm{NO}(v=2 \rightarrow 2)$ is examined. Arrival time distributions of $\mathrm{NO}(v=2 \rightarrow 2, J=5.5)$ were acquired along a wedge of $0-7.2 \mathrm{ML} \mathrm{Ag} / \mathrm{Au}(111)$. This data set was chosen because it showed scattering behavior for a rather broad thickness range, still offering good thickness resolution ( $0.3 \mathrm{ML}$ positioning error). However, a measurement from a wedge with a smaller gradient (0-3 ML) and therefore higher resolution reproduces observed behavior.

The TOF profiles were fitted following the procedure described in Appendix B. Raw data (circles) and the corresponding fit functions (solid lines) are presented in Figure 4.1. Depending on the film thickness, the data can be separated into three groups: The first three shapes (Figure 4.1, top panel) show NO scattered off positions with clean $\mathrm{Au}(111)$. As expected for a common surface, the TOF shapes are equal sharing the peak at $11.0 \mu \mathrm{s}$. For film thicknesses between $0-3 \mathrm{ML} \mathrm{Ag} / \mathrm{Au}$, a peak of the distributions is shifted towards later arrival times (Figure 4.1, central panel). Additionally, a slight broadening in time can be observed. This range of thicknesses can be regarded as a transition between $\mathrm{Au}$ and $\mathrm{Ag}$. Finally, above $3 \mathrm{ML} \mathrm{Ag} / \mathrm{Au}$, the data does not exhibit further change and constant TOF distributions are obtained (Figure 4.1, bottom panel). All shapes share the same width in time and a peak at $13.5 \mu \mathrm{s}$. Due to this constant behavior, this film thickness of $3 \mathrm{ML}$ will be referred to as the "bulk limit" concerning the translational inelasticity.

The overall good agreement shows that the model is suitable for the description of data. The corresponding translational energy distributions derived from those fits is depicted in Figure 4.2. In a similar manner as in the TOF distributions, the gradual shift of the kinetic energy ( $0.32 \mathrm{eV}$ to $0.24 \mathrm{eV})$ can be observed in the range 0-3 ML Ag/Au. One additional information is the FWHM of the energy distributions which is approximately constant independent of the film thickness. 

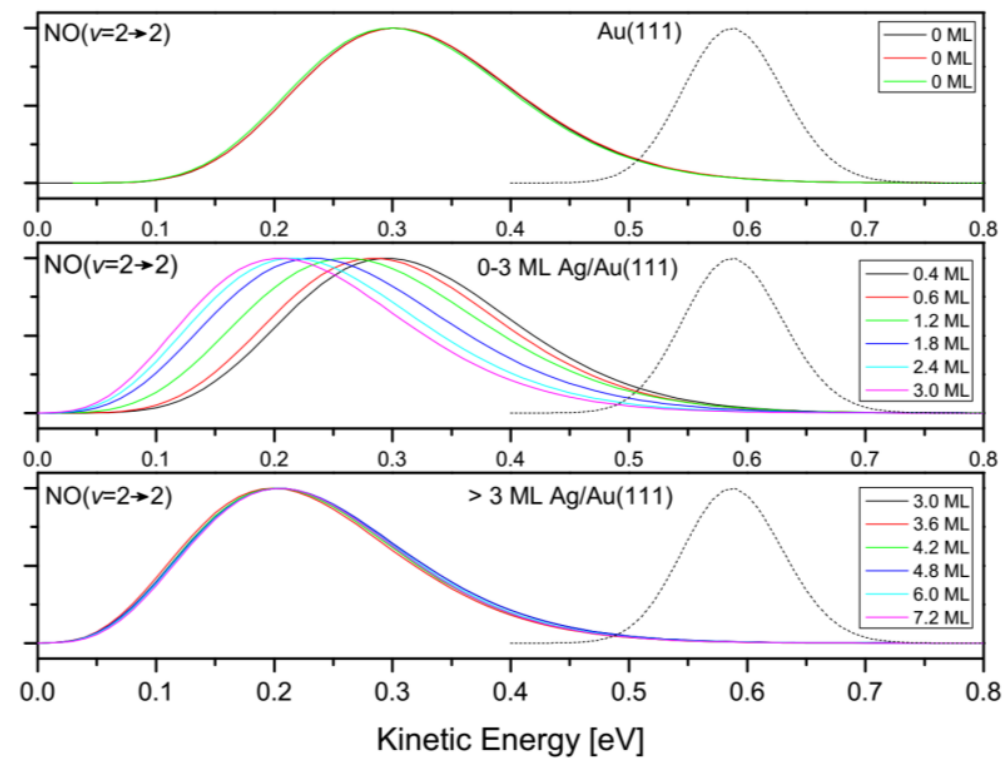

Figure 4.2 Kinetic energy distributions of $\mathrm{NO}(v=2 \rightarrow 2)$ derived from the fits on the data depicted in Figure 4.1 and presented in an analogous manner. The transition from Au to Ag exhibits an energy shift of the distribution by $0.1 \mathrm{eV}$. The dotted curve describes the kinetic energy distribution of the incident beam.
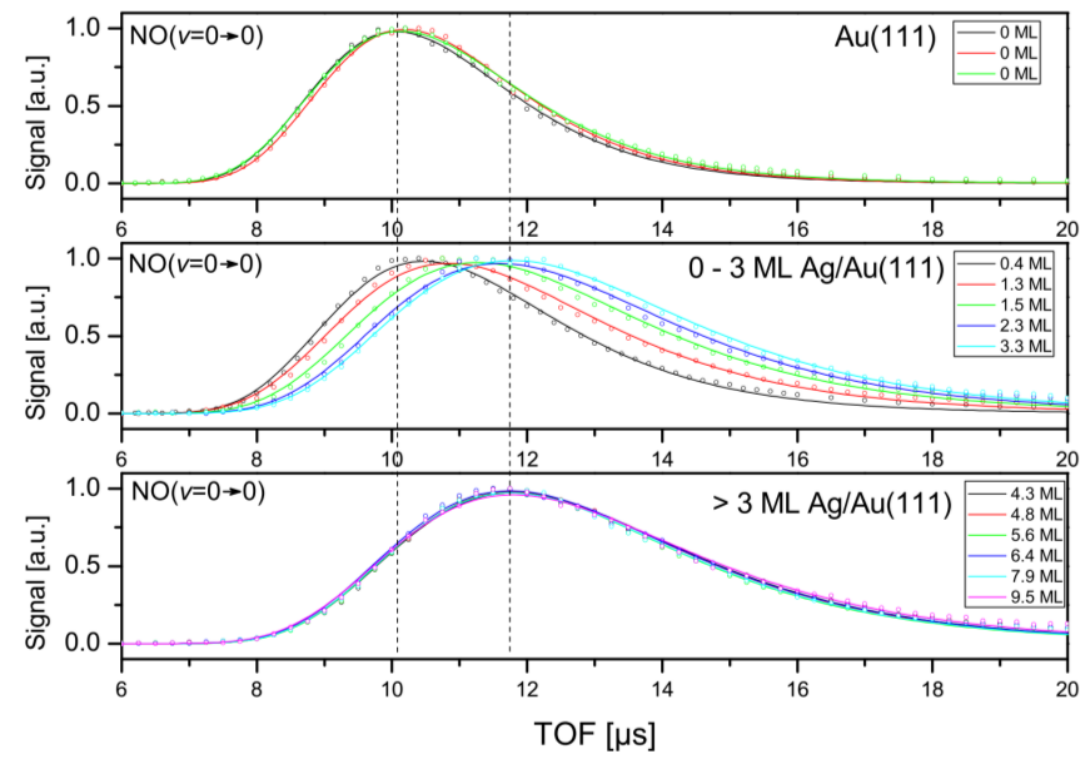

Figure 4.3: Arrival time distributions of $\mathrm{NO}(v=0 \rightarrow 0, J=6.5)$ scattering from 0-10 ML Wedge Ag/Au(111). Peak timing of both limiting cases (clean Au and bulk limit of $\mathrm{Ag} / \mathrm{Au}$ ) are marked by two dashed vertical lines.

To compare both vibrational elastic scattering cases, arrival time distributions of $\mathrm{NO}(v=0 \rightarrow 0, J=6.5)$ have been obtained in a similar manner as for $\mathrm{NO}(v=2 \rightarrow 2, J=5.5)$. The data sets can be grouped in an analogue manner and is depicted in Figure 4.3. Neither the TOF distributions nor the corresponding translational energy distributions (Figure 4.4) show a significant difference to the previously discussed $\mathrm{NO}(v=2 \rightarrow 2)$ scattering behavior. 

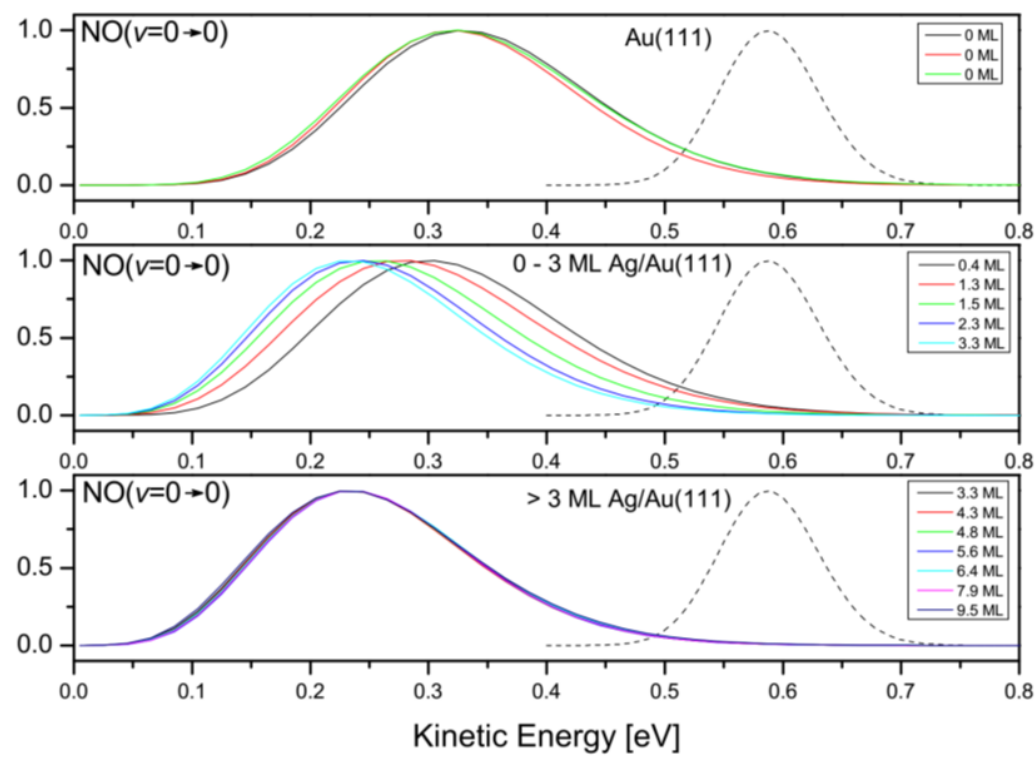

Figure 4.4: Translational Energy Distributions of $\mathrm{NO}(v=0 \rightarrow 0, J=6.5)$ scattering from 0-10 ML Wedge Ag/Au(111). The distribution of the incoming beam is depicted by a dashed line.

The best way to present both scattering channels in one plot is to plot the mean final kinetic energy against the film thickness. The result is depicted in Figure 4.5. From this plot, we see that the energy change in the transition region (0-3 ML Ag/Au) can be approximated as a linear decrease.

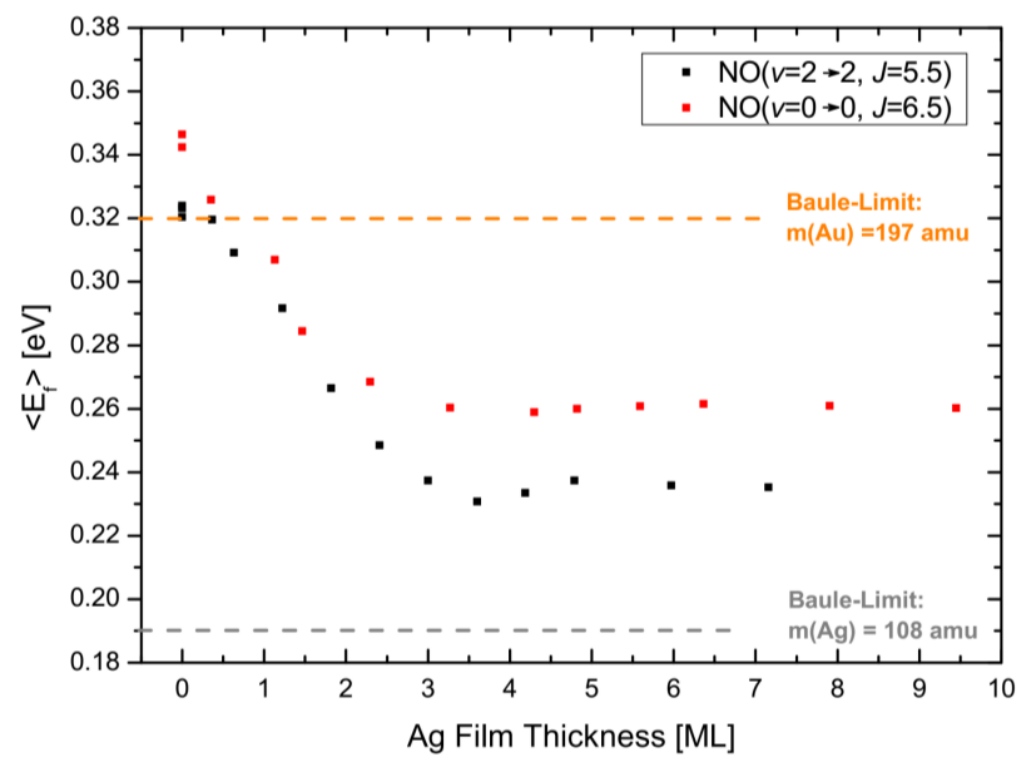

Figure 4.5: Final mean translational energy of $\mathrm{NO}(v=2 \rightarrow 2, J=5.5)$ and $\mathrm{NO}(v=0 \rightarrow 0, J=6.5)$. As a reference, the Baule limit (see text) the mass of a single crystals of $\mathrm{Au}$ and $\mathrm{Ag}$ are marked with dashed lines.

For previous studies of molecular beam scattering from a single metal surface, a simple hard cube model assuming the head-on collision of a NO molecule of mass $m_{\text {No }}$ with a single surface atom $m_{\text {surf }}$ was successful in describing vibrational elastic scattering. From linear momentum and energy conservation, the ratio between final and incidence translational energy is derived:

$$
\frac{\left\langle E_{f}\right\rangle}{\left\langle E_{i}\right\rangle}=\frac{\left(m_{\mathrm{NO}}-m_{\mathrm{Surf}}\right)^{2}}{\left(m_{\mathrm{NO}}+m_{\mathrm{Surf}}\right)^{2}}
$$


According to the molecular $\left(m_{\mathrm{NO}}=30 \mathrm{amu}\right.$ ) respectively surface mass ( $m_{\mathrm{Au}}=197 \mathrm{amu}$ and $\left.m_{\mathrm{Ag}}=108 \mathrm{amu}\right)$ and the investigated incidence energy $\left(E_{\mathrm{i}}=0.59 \mathrm{eV}\right)$, the model predicts a final kinetic energy of $0.32 \mathrm{eV}$ respectively $0.19 \mathrm{eV}$. As a reference, those value which are often referred to as "Baule limit", are marked in Figure 4.5 as well. Both measurements were taken with slightly different experimental configuration explaining the offset; within the experimental errors both dataset are equal. Comparing the Baule limit with the obtained kinetic energy, very good agreement is achieved in the case of $\mathrm{Au}(111)$. Assuming bulk behavior for $>3 \mathrm{ML} \mathrm{Ag} / \mathrm{Au}$, although the model slightly underestimates the experimental value giving still good agreement.

\subsubsection{Inelastic $\mathrm{NO}(v=2)$ Scattering - Thickness Dependence $\mathrm{NO}(v=2 \rightarrow 1)$}

Apart for the previously presented examples of elastic scattering, TOF distributions have been acquired for the vibrationally inelastic channel $\mathrm{NO}(v=2 \rightarrow 1, J=5.5)$. Because this data was measured together with $\mathrm{NO}(v=2 \rightarrow 2, J=5.5)$ using the same experimental configurations, those sets provide very good conditions for a direct comparison. For a few selected film thicknesses, the arrival time distributions are juxtaposed in Figure 4.6. As in previous plots, the raw data (circles) is shown with a corresponding fit function (lines) according to the procedure described in Appendix B.

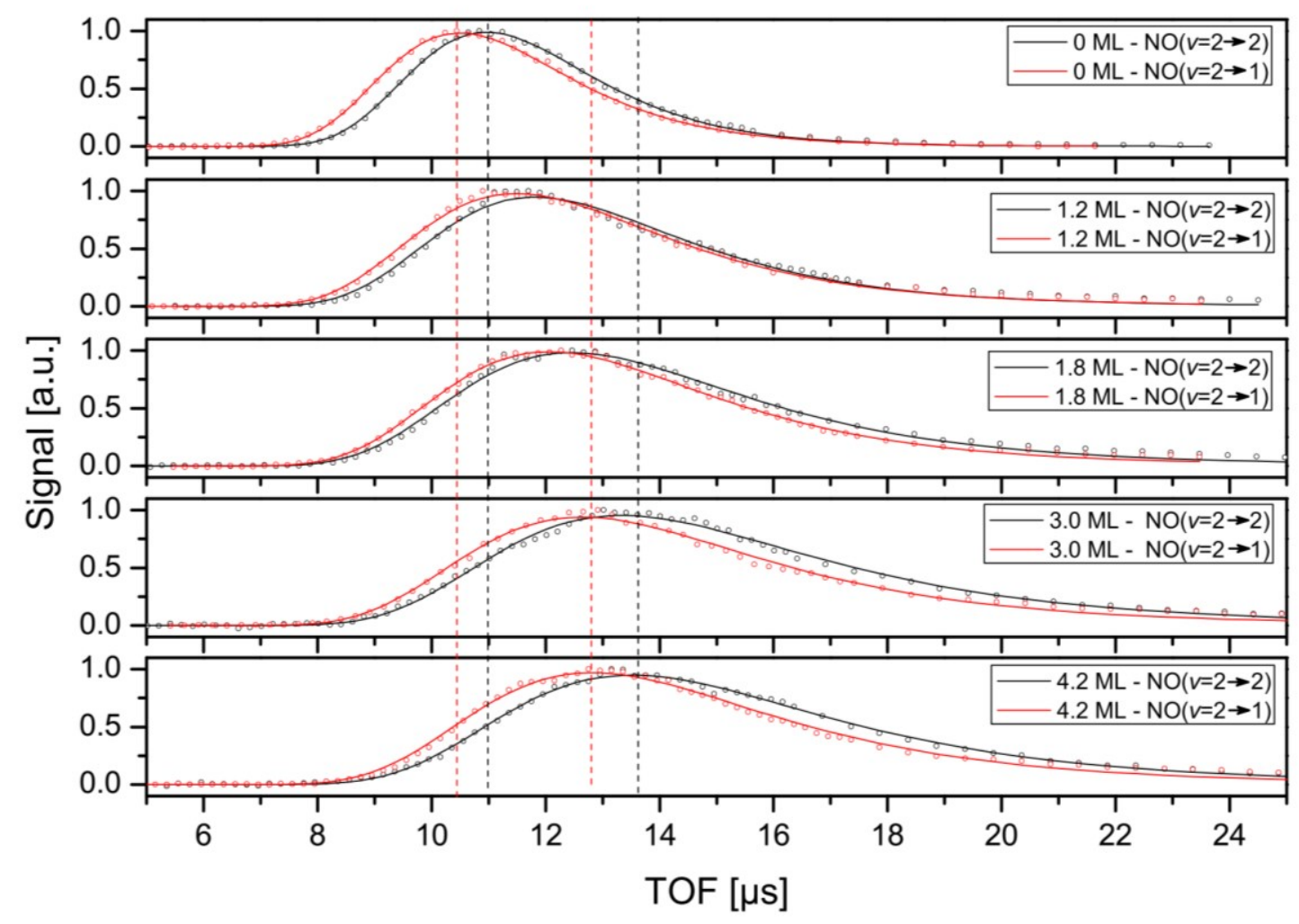

Figure 4.6: Selected arrival time distributions of $\mathrm{NO}(v=2 \rightarrow 2)$ and $\mathrm{NO}(v=2 \rightarrow 1)$. The peak timings for clean Au and the bulk limit of $3 \mathrm{ML} \mathrm{Ag/Au}$ are marked by dashed lines for both channels.

It is observed for all film thicknesses that the peak of the $\mathrm{NO}(v=2 \rightarrow 1)$ TOF distribution is shifted towards earlier arrival times in comparison with the corresponding $\mathrm{NO}(v=2 \rightarrow 2)$ distribution. In the literature, this effect is describe as vibration-translation (VT) coupling [87]: When a molecule is undergoing vibrational relaxation, a part of the released energy is converted into translational energy, leading to earlier arrival times in the arrival time distribution. The dashed lines in Figure 4.6 are marking 
the peak of the elastic (black) and the inelastic channel (red) for the limiting cases of a clean Au surface and $3 \mathrm{ML} \mathrm{Ag} / \mathrm{Au}$, displaying a shift in the order of $\sim 0.5 \mu \mathrm{s}$. Aside from that fact, all characteristics observed for the vibrationally elastic case like broadening in time and the gradual peak shift between 0-3 ML Ag/Au can observed for the vibrationally inelastic scattering case. Accordingly, the mean final translational energy is constant from a film thickness of $3 \mathrm{ML}$ on as can be seen in Figure 4.7.

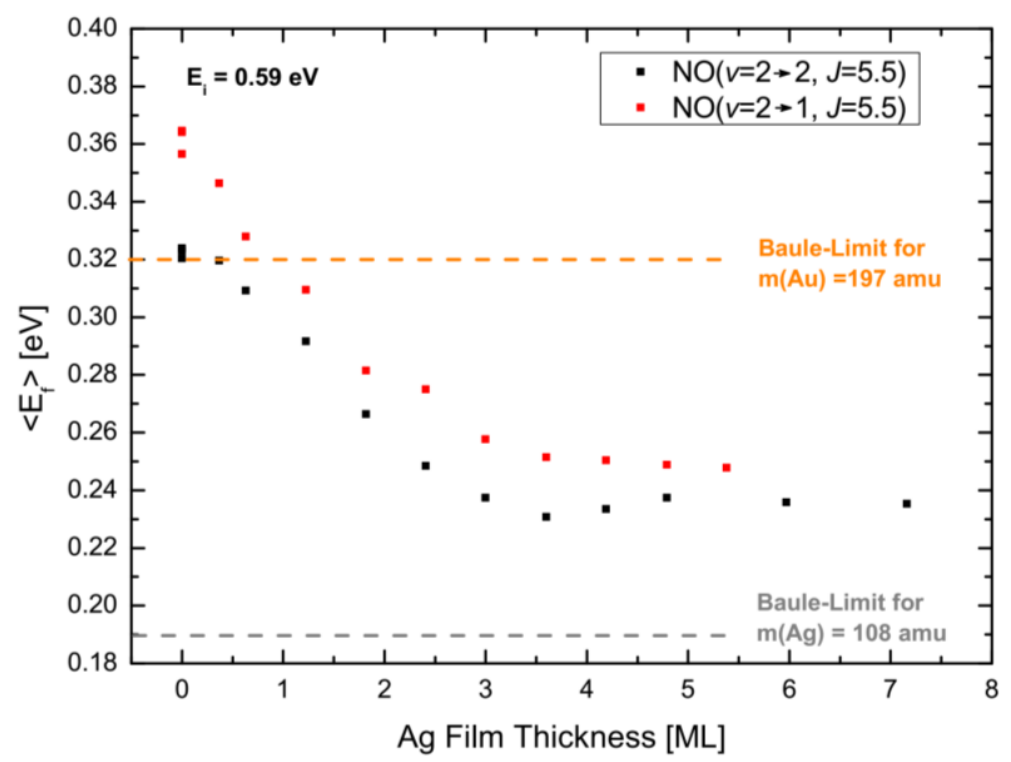

Figure 4.7: Final mean translational energy of the scattered molecules in the scattering channels of $N O(v=2 \rightarrow 2)$ and $\mathrm{NO}(v=2 \rightarrow 1)$. According to a simple model ("Baule limit", eq. (4.1)), the expected values for a single collision from Au(111) and $\mathrm{Ag}(111)$ are calculated and noted as dashed lines.

\subsection{3 $\mathrm{CO}(v=2)$ Scattering - Thickness Dependence $\mathrm{CO}(v=2 \rightarrow 2,1)$}

A similar diatomic molecule with almost identical mass is $\mathrm{CO}$ which was used as second scattering target. Analogue to the measurement procedure in the previous sections, arrival time distributions were acquired for the scattering of vibrationally excited $\mathrm{CO}(v=2)$. Most of the measurements were carried out with the $6 \% \mathrm{CO} / \mathrm{H}_{2}$ mixture $\left(\mathrm{E}_{\mathrm{i}}=0.64 \mathrm{eV}\right)$, an incidence energy comparable to the previously described NO mixture. $\mathrm{CO}(v=2 \rightarrow 2)$ arrival time distributions were obtained by scanning along a wedge of 0-10 ML Ag/Au with an error of 0.4 ML. The results are presented in Figure 4.8. 


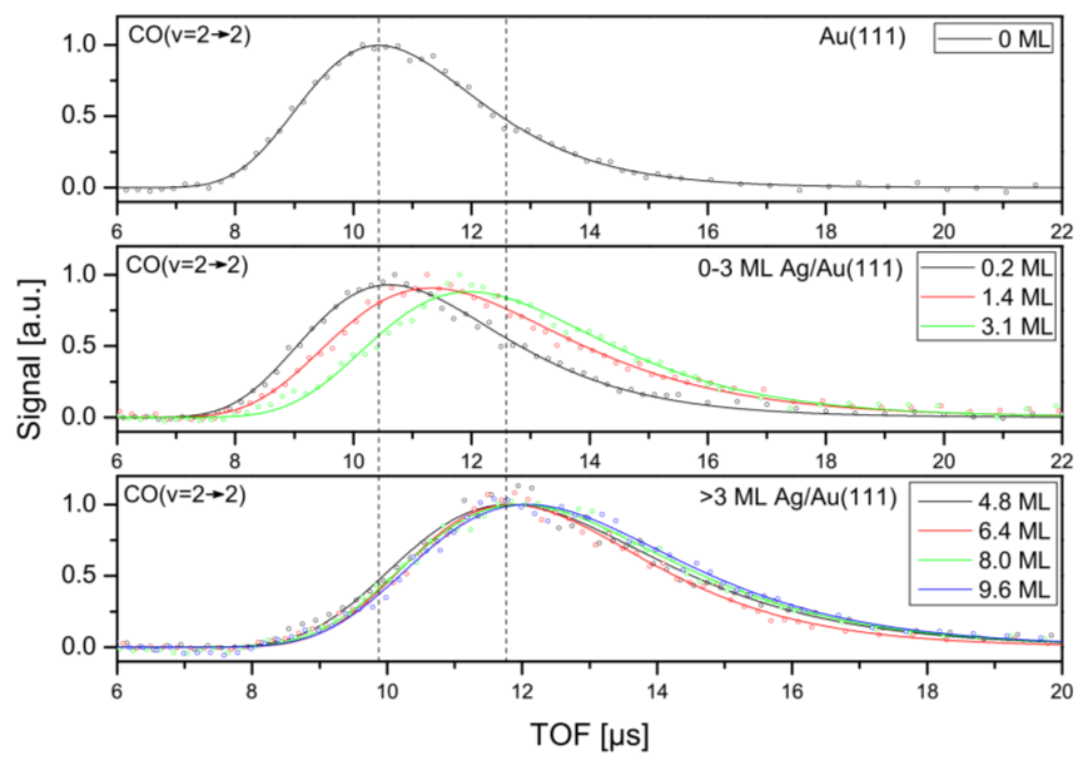

Figure 4.8: Arrival time distributions of $\mathrm{CO}(v=2 \rightarrow 2)$ scattering from 0-10 ML Wedge Ag/Au(111). Peak timing of both limiting cases (clean Au and bulk limit of $\mathrm{Ag} / \mathrm{Au}$ ) are marked by two dashed vertical lines.

The data was grouped in the same manner because a similar behavior as for the scattering of NO is observed: Between 0-3 ML Ag/Au, the peak of the distribution is shifting towards later arrival times before reaching a constant behavior for thicknesses above this bulk limit. All in all, the trend of the $v=2 \rightarrow 2$ scattering channels of $\mathrm{NO}$ and $\mathrm{CO}$ with film thickness shows good agreement.

However, differences between the two molecules can be observed when juxtaposing the TOF shapes of the vibrationally elastic and inelastic channels. Figure 4.9 depicts $\operatorname{CO}(v=2 \rightarrow 2,1)$ arrival time distributions for a few selected film thicknesses. Note the dashed lines marking the peak of the distribution for the elastic (black) and inelastic (red) channel: For clean $A u(111)$, those marks are indistinguishable within the experimental resolution, the both TOF shapes are identical (Figure 4.9, top panel). This is a stark contrast to scattering of $\mathrm{NO}$ on $\mathrm{Au}(111)$ (see Figure 4.6, top panel) where the $\mathrm{NO}(v=2 \rightarrow 1)$ TOF distribution was shifted to earlier peak timings compared to $\mathrm{NO}(v=2 \rightarrow 2)$ as a result of VT coupling. This coupling seems to be absent for this gas-surface system. However, when silver is deposited to the surface, both TOF shapes exhibit a clear difference: the inelastic $\mathrm{CO}(v=2 \rightarrow 1)$ scattering channel is peaking $\sim 0.5 \mu$ s earlier in comparison with its elastic counterpart as visualized by the separated dashed lines (Figure 4.9, lower panels). 

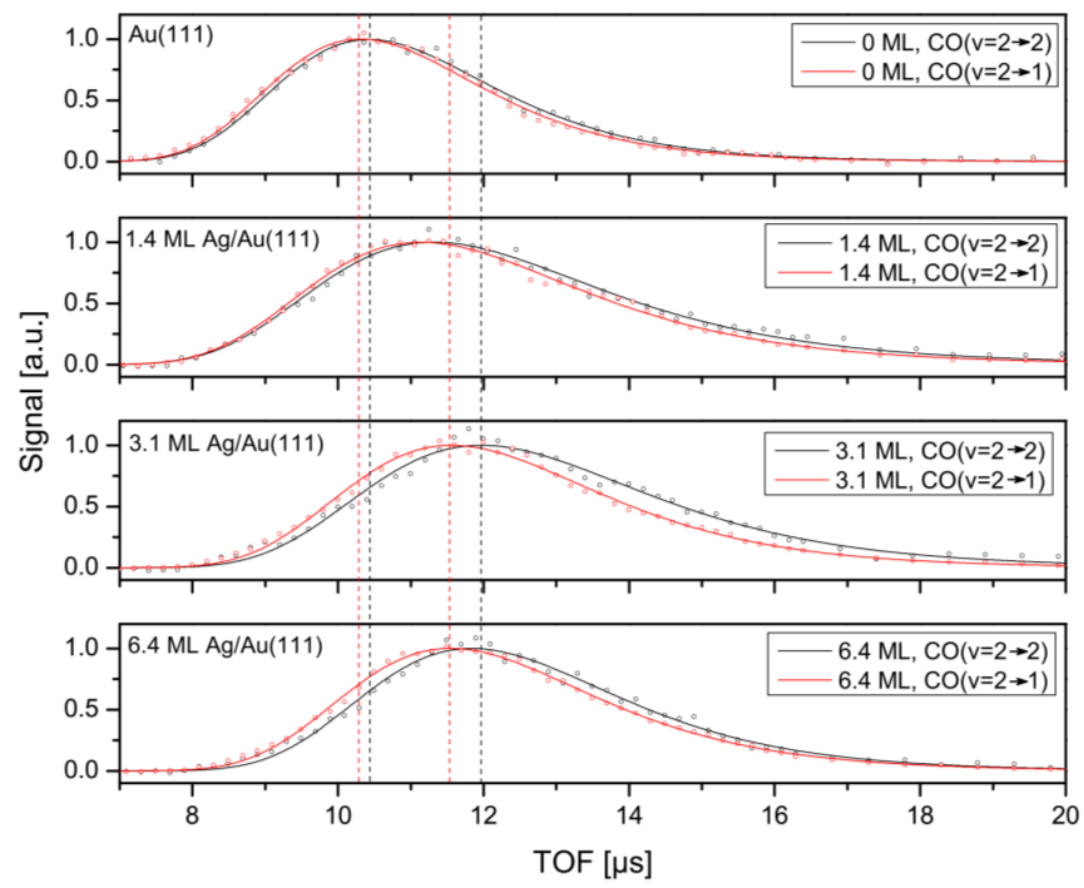

Figure 4.9: Selected arrival time distributions of $\mathrm{CO}(v=2 \rightarrow 2)$ (black) and $\mathrm{CO}(v=2 \rightarrow 1)$ (red). The peak timings for clean $\mathrm{Au}$ and the bulk limit of $3 \mathrm{ML} \mathrm{Ag/Au}$ are marked by dashed lines for both channels in the corresponding color.

To visualize how the scattering characteristics evolve quantitatively with amount of silver on the surface, the final mean translational energy is calculated and presented as a function of film thickness (Figure 4.10). The qualitative resemblence to the scattering of NO is astonishing: Within the first monolayers, a decrease is observed before reaching a constant value unchanging with increasing film thickness.

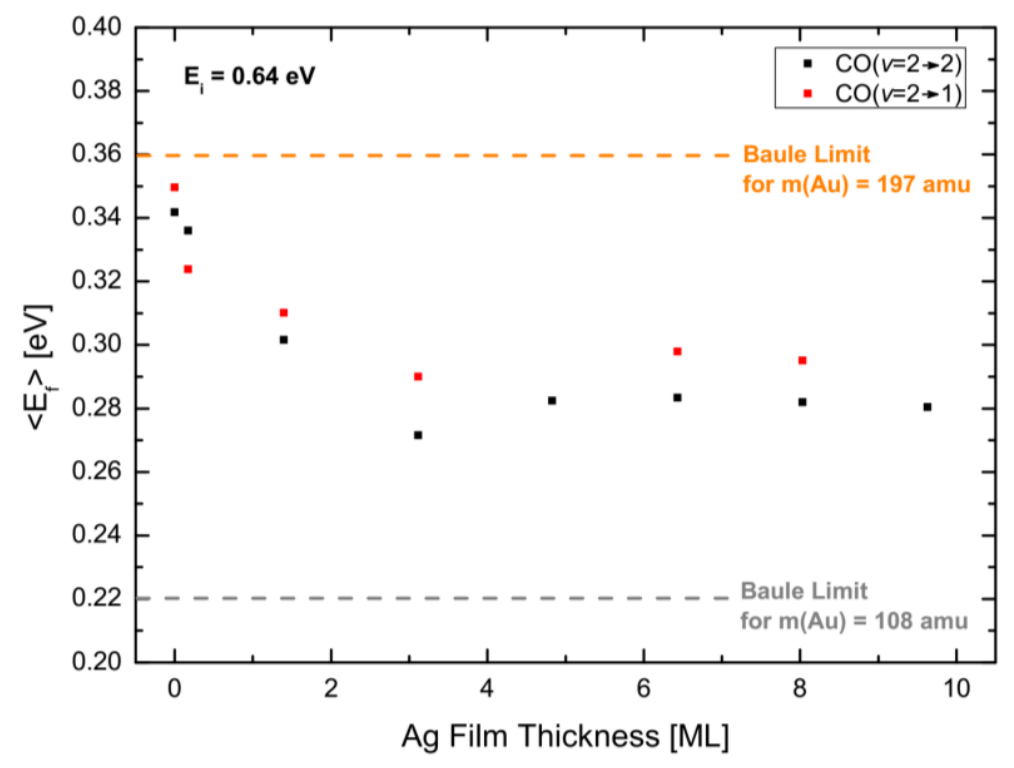

Figure 4.10: Final mean translational energy of the scattered molecules in the scattering channels of $\mathrm{CO}(v=2 \rightarrow 2)$ and $\mathrm{CO}(v=2 \rightarrow 1)$. The "Baule-limit" (eq. 4.1) for a single collision with Au(111) and Ag(111) are calculated and noted as dashed lines.

As it was utilized earlier for NO, the expected translational energy after $\mathrm{CO}$ scattering with a single surface atom of $\mathrm{Au}\left(\mathrm{E}_{\mathrm{f}, \mathrm{Baule}}=0.36 \mathrm{eV}\right)$ and $\mathrm{Ag}\left(\mathrm{E}_{\mathrm{f}, \mathrm{Baule}}=0.22 \mathrm{eV}\right)$ was calculated according to a simple model (see 4.1.1, eq. 4.1) and is shown as dashed lines. Comparing those values with the elastic 
channel $\mathrm{CO}(v=2 \rightarrow 2)$ reveals very good agreement for a clean $\mathrm{Au}(111)$ surface; the calculated value for silver film thicknesses $>3 \mathrm{ML}$ slightly underestimates the experimental data, giving yet good agreement.

\subsection{Discussion}

The key findings of the NO and CO TOF experiments are the following: Both molecules show in all investigated vibrational states the same trend with film thickness. The final mean kinetic energy is shifting within the formation of the first 2-3 monolayers. From that film thickness on, the obtained translational energy stays constant. A difference between $\mathrm{NO}$ and $\mathrm{CO}$ is observed when juxtaposing vibrationally elastic and inelastic scattering channel: in the case of NO, the $v=2 \rightarrow 1$ channel is exhibiting higher kinetic energy for all film thicknesses, a phenomenon described as V-T coupling. However, for $\mathrm{CO}$, both channels cannot be distinguished on $\mathrm{Au}(111)$ but can be experimentally separated for scattering from silver films of $3 \mathrm{ML} \mathrm{Ag/Au}$.

First, the general characteristics of the common trend in thin-film scattering will be discussed for both observed vibrationally elastic scattering channels of NO including the comparison with data scattered off bulk samples of $\mathrm{Au}(111)$ and $\mathrm{Ag}(111)$. Second, $\mathrm{NO}$ and $\mathrm{CO}$ will be compared by focusing on the effective surface mass that those molecules experience during the collision. The difference in V-T coupling will be treated in the context of nonadiabatic interactions. Finally, a possible mechanism explaining the scattering trend with film thickness is proposed.

\subsubsection{Vibrationally Elastic Scattering of $\mathrm{NO}(v=2 \rightarrow 2)$ and $\mathrm{NO}(v=0 \rightarrow 0)$}

Before interpreting the thickness trend, the scattering behavior from a thin film needs to be characterized and compared with data obtained from a bulk sample. Can differences be found between a thin film of silver and a bulk $\mathrm{Ag}(111)$ surface? On the basis of several indicators, information about the film quality can be obtained.

First conclusions can already be drawn from the observation of constant behavior from film thicknesses above $3 \mathrm{ML} \mathrm{Ag/Au}$. Because the growth of three-dimensional structures would result in surface roughening and therefore broadening of the TOF distributions, this observation is a further corroboration for the layer-by-layer growth of the Ag/Au system. A more detailed look is possible via the width of the translational energy distribution which is depicted for the elastic channels in Figure 4.11. The approximately constant behavior independent of the film thickness supports the hypothesis of a flat surface. In a previous set of experiments of $\mathrm{NO}(v=0 \rightarrow 0)$ scattering from bulk samples from $\mathrm{Au}(111)$ and $\mathrm{Ag}(111)$, a similar feature was found: TOF distributions were recorded with incidence energies close to the one used in the present work. It was found that after scattering, the absolute width of the resulting translational energy distribution was the equal independent of the noble metal surface. A direct comparison of the between the data of this work and the bulk data can be found in Appendix $C$. 


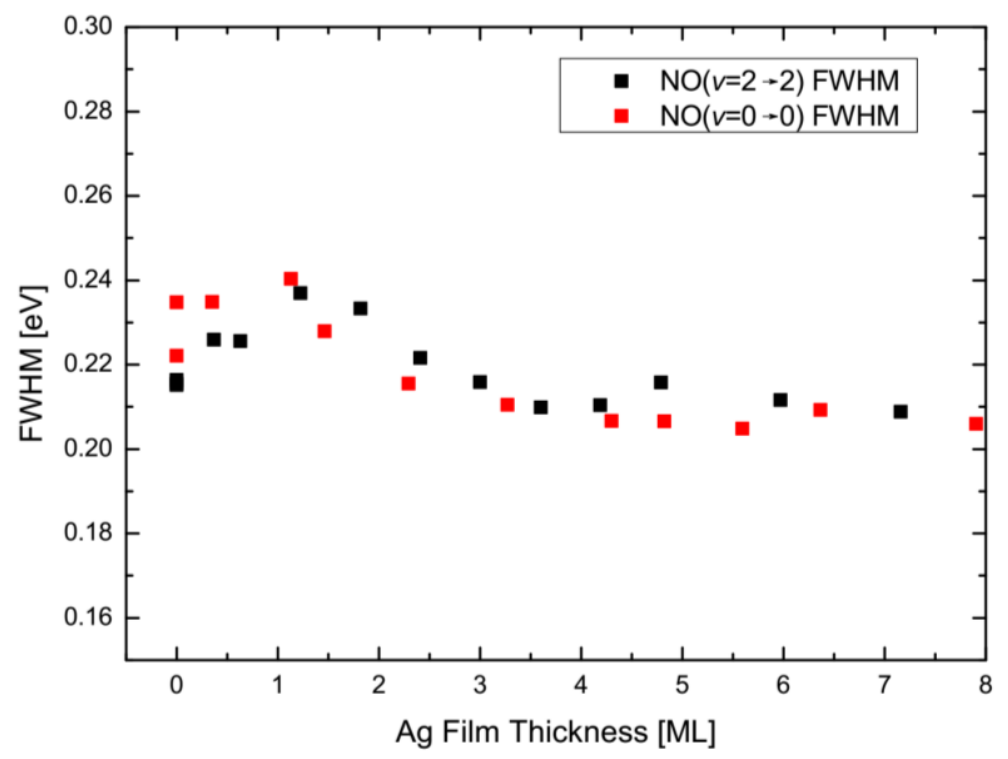

Figure 4.11: Energetic width (full width at half maximum) of the translational energy distributions (see Figure 4.2, Figure 4.4) for both vibrationally elastic scattering channels.

A second parameter of interest in this context is the width of angular distributions. For a corrugated or rough surface it is expected that this measure will be significantly broadened in comparison to a flat metal surface. The distributions of $\mathrm{NO}(v=2 \rightarrow 2)$ and $\mathrm{NO}(v=2 \rightarrow 1)$ scattered from Au and thin silver film surfaces however show narrow width similar to those obtained from $\mathrm{NO}(v=0 \rightarrow 0)$ from bulk samples (see Appendix C).

All in all, NO scattered off a film of $\mathrm{Ag} / \mathrm{Au}(111)$ does not show significant differences to scattering from a $\mathrm{Ag}(111)$ surface. It is therefore possible to conclude that all investigated surfaces are atomically flat and can be compared to an annealed surface. Furthermore we assume that concerning the translational energy transfer, from a thickness of $3 \mathrm{ML}$ onwards, a $\mathrm{Ag} / \mathrm{Au}$ thin film surfaces acts like a $\mathrm{Ag}(111)$ surface, allowing the use of the term "bulk-limit". With $1 \mathrm{ML} \mathrm{Ag/Au(111)} \mathrm{corresponding} \mathrm{to} \mathrm{a}$ thickness of $2.357 \AA$ [80], it is remarkable that a film with a thickness of less than two lattice constants $\left(d_{A u}=4.065 \AA[52]\right)$ is sufficient to be "felt" as a bulk silver crystal by the NO molecule.

Let us return to the question how the properties of thin films $\mathrm{Ag} / \mathrm{Au}$ are different in comparison to a bulk $\mathrm{Ag}(111)$ crystal. There is indeed a feature limited to the electronic structure of thin films: Photoelectron spectroscopy revealed the existence of localized electronic states close to the Fermi level present above $7 \mathrm{ML} \mathrm{Ag} / \mathrm{Au}$ [10]. In a film of $\mathrm{Ag} / \mathrm{Au}$, due to a limited film thickness the movement of electrons is confined in one direction. Do films with that thickness range show a different beam scattering behavior? The sample with the biggest film thicknesses subject to investigation in this work contained a wedge of up to $33 \mathrm{ML} \mathrm{Ag/Au(111).} \mathrm{As} \mathrm{it} \mathrm{turned} \mathrm{out,} \mathrm{translational} \mathrm{energies} \mathrm{obtained} \mathrm{after}$ scattering from higher thicknesses exhibit the same result as in the range of 3-10 ML Ag/Au. All in all, for the observed samples, no difference between a thin film above $3 \mathrm{ML} \mathrm{Ag/Au}$ and a bulk $\mathrm{Ag}(111)$ crystal is found (Figure 4.12). It is concluded that translational energy transfer is therefore mainly governed by mechanical properties which are common to both kind of surfaces. 


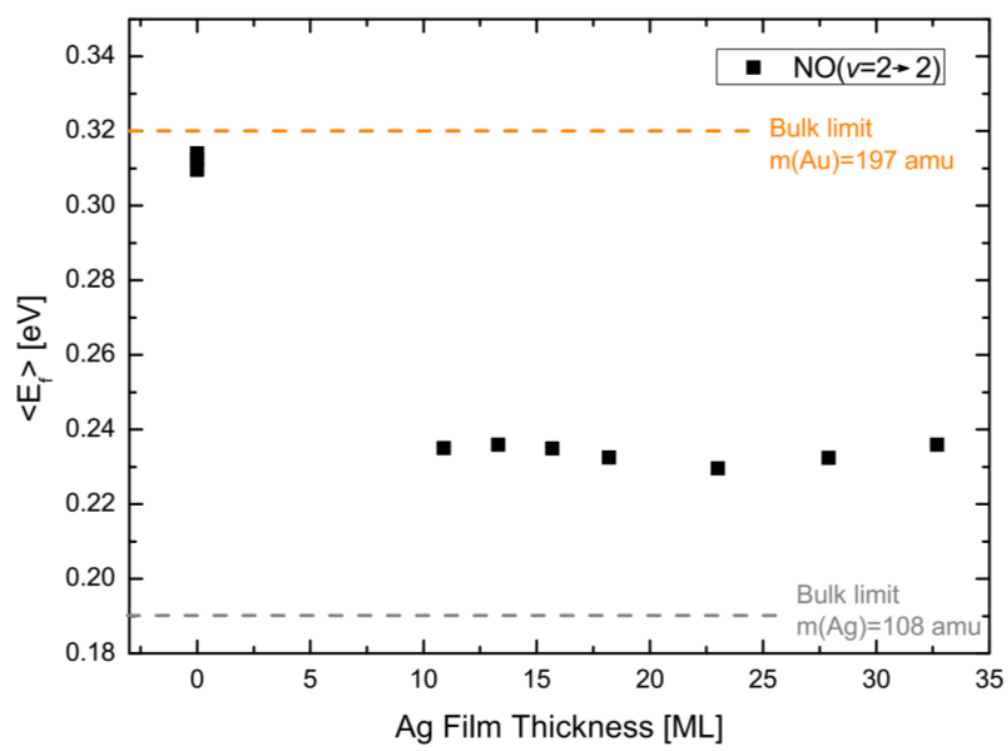

Figure 4.12: Influence of higher film thicknesses: Final translational mean energy of $N O(v=2 \rightarrow 2)$ scattering from Au and silver films up to $33 \mathrm{ML} \mathrm{Ag/Au}$. Constant behavior is observed above $10 \mathrm{ML}$ consistent with previous results (Figure 4.5).

\subsubsection{Probing the Effective Mass of $\mathrm{NO}(v=2 \rightarrow 2)$ vs $\mathrm{CO}(v=2 \rightarrow 2)$}

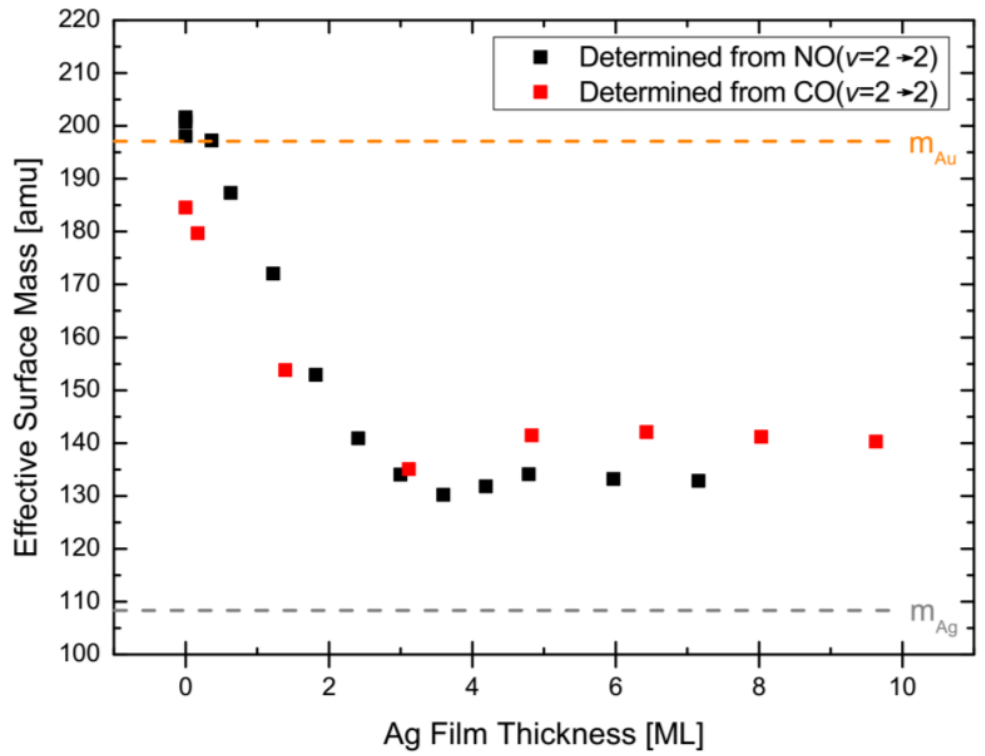

Figure 4.13: Effective mass of the surface determined from the mean translational energy of scattered NO and CO according to equation (4.1). As a reference, atomic masses of Au and Ag are marked as dashed lines.

Similarly as in the case of scattering from single crystal, the model of head-on collision between a molecule and a single surface atom (eq. 4.1) was used successful in predicting a translational energy after scattering from clean $\mathrm{Au}(111)$ and films above $3 \mathrm{ML} \mathrm{Ag} / \mathrm{Au}(111)$ for $\mathrm{NO}(v=2 \rightarrow 2)$ (Figure 4.5, Figure 4.7) and $\mathrm{CO}(v=2 \rightarrow 2)$ (Figure 4.10). In a slightly different approach, this model can be employed to calculate an effective surface mass $m_{\text {surf }}$ from the acquired final translational energy. This picture (Figure 4.13) provides information about what kind of surface is "felt" by the molecule during the collision: For both bulk cases ( $\mathrm{Au}$ and $3 \mathrm{ML} \mathrm{Ag} / \mathrm{Au}$ ), the effective mass can be compared to the atomic mass of a single surface atom by calculating the ratio $\frac{m_{\mathrm{eff}}}{m_{\mathrm{Au}}}$ and $\frac{m_{\mathrm{eff}}}{m_{\mathrm{Ag}}}$. This ratio can be interpreted as the number of involved surface atoms $N_{\text {eff }}$ during the molecular collision. The results determined from 
both molecules show good agreement on $\mathrm{Au}\left(N_{\mathrm{eff}, \mathrm{NO}}=1.0, N_{\mathrm{eff}, \mathrm{CO}}=0.9\right)$ and $\mathrm{Ag}\left(N_{\text {eff, NO }}=1.2, N_{\text {eff,CO }}=1.3\right)$, suggesting that the observed process of translational energy transfer for vibrationally elastic scattering does not depend on the employed scattering object. Although quantitatively a slightly better agreement can be found for Au than Ag, in both cases $N_{\text {eff }}$ is close to unity. That supports the envisioned model, a purely mechanical picture is therefore highly suggestive and will outlined in more detail in the final section of this chapter.

The obtained data for NO is very consistent with previously acquired vibrationally elastic scattering data: $\mathrm{NO}(v=3 \rightarrow 3)$ scattering from $\mathrm{Au}(111)$ has been measured on the same apparatus for nine different incidence energies [88]. $N_{\text {eff }}$ was obtained in a range of 1.1-1.6 which is very consistent with the results of this work. Additionally, $\mathrm{NO}(v=0 \rightarrow 0)$ scattering data from bulk $\mathrm{Au}(111)$ and bulk $\mathrm{Ag}(111)$ has been acquired in our group. For higher incidence energies $(0.36 \mathrm{eV}$ to $0.92 \mathrm{eV}) N_{\text {eff }}$ would range from 1.4-2.8 (Au), respectively 1.5-2.1 (Ag) (see Appendix C). Since there is no dependency of $N_{\text {eff }}$ on the incidence energy, the range of values obtained from those measurements are a good ballpark number for the uncertainty of this experiment.

$\mathrm{CO}(v=0 \rightarrow 0)$ scattering data was acquired for incidence energies close to the one used in this work $\left(0.6 \mathrm{eV}\right.$ ) on both bulk samples (see Appendix D). The number of involved surface atoms is $N_{\text {eff }}=1.4(\mathrm{Au})$ respectively $1.9(\mathrm{Ag})$. Generally, it is observed that our data of $N_{\text {eff }}$ for $\mathrm{Au}$ and $\mathrm{Ag}$ is at the lower end of the previously measured range, a fact that can be explained by slight systematic errors of the flight path. Apart from that, all in all, there is good agreement with the previously acquired data, supporting the assumption that scattering from a crystal of thicknesses $>3 \mathrm{ML} \mathrm{Ag} / \mathrm{Au}$ is equal to scattering from bulk $\mathrm{Ag}(111)$.

\subsubsection{Vibration-Translation (V-T) Coupling of NO and CO on Au(111) and Ag/Au(111)}

One remarkable phenomenon was discovered in the investigation of molecules changing their vibrational state during the collision with a surface: when gaining/losing vibrational energy in the scattering process it was found that they are leaving the surface with lower/higher velocity in comparison with those scattered in a vibrationally elastic manner. Partially, translational energy is converted into vibrational energy during vibrational excitation and vice versa in a vibrational relaxation process. This effect, the vibration-translation (V-T) coupling, was observed in recent years for several gas/metal systems like NO/Au $[87,88], \mathrm{NO} / \mathrm{Ag}[48]$ and $\mathrm{HCl} / \mathrm{Au}[89]$. The most detailed study about this aspect was carried out for $\mathrm{NO}(v=3 \rightarrow 2, v=3 \rightarrow 1)$ scattering from $\mathrm{Au}(111)$ for several incidence energies, finding a correlation between the incidence energy and the translational energy increase in the inelastically scattered channel [88]. For $\mathrm{HCl}(v=2 \rightarrow 1)$ scattering from $\mathrm{Au}(111)$ however, this effect was absent.

In this work, one incidence energy $(0.6 \mathrm{eV})$ is employed to investigate the influence of the surface on $\mathrm{V}$-T coupling for two different molecules $\mathrm{NO}$ and $\mathrm{CO}$. Since the effect is at the edge of the experimental resolution, Ag films above $3 \mathrm{ML} \mathrm{Ag/Au} \mathrm{will} \mathrm{be} \mathrm{regarded} \mathrm{as} \mathrm{a} \mathrm{Ag}(111)$ bulk sample, providing in a number of measurement points for $\mathrm{Ag}$. In direct comparison of $\mathrm{Au}$ and $\mathrm{Ag}$, a statistical analysis allows a quantitative estimation and will be the base for error calculation. Because the TOF measurements were carried out from the same surface, the same mixture and the same day, systematic errors (due to changes of the flight length) can be minimized. 


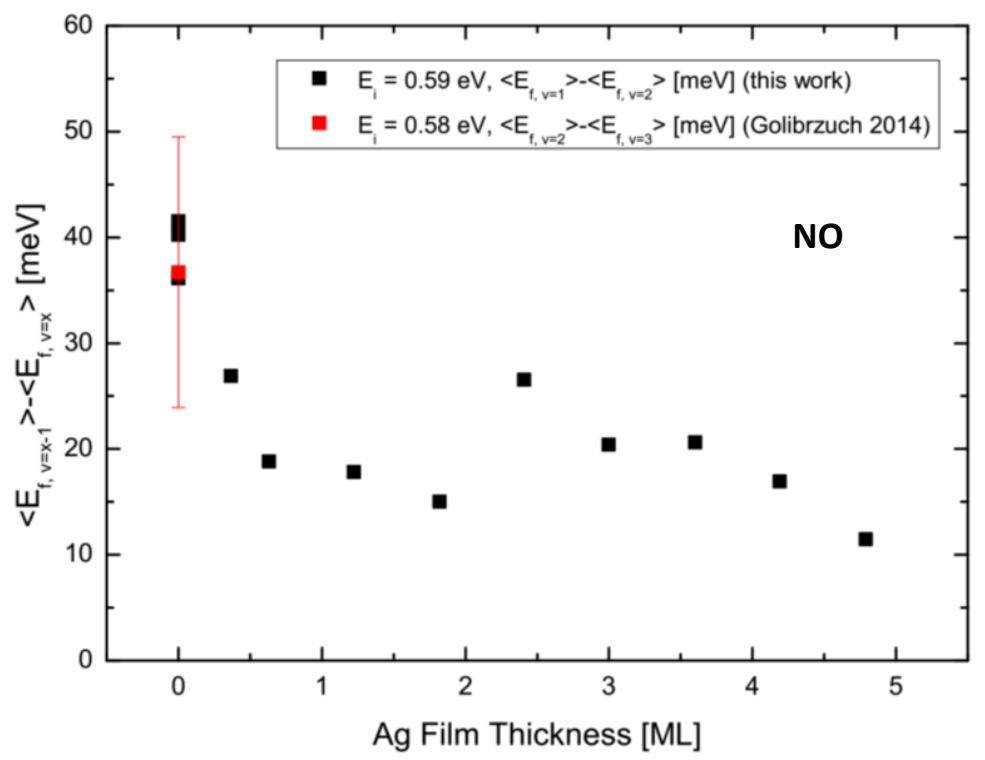

Figure 4.14: Kinetic energy difference between $N O(v=2 \rightarrow 1)$ and $N O(v=2 \rightarrow 2) . E_{i}=0.59 \mathrm{eV}$. For $A u(111)$, a point of a previous energy difference measurement between $\mathrm{NO}(v=3 \rightarrow 2)$ and $\mathrm{NO}(v=3 \rightarrow 3)$ from Ref. [88] is included.

From the final mean translational energies (Figure 4.7 and Figure 4.10), the difference between inelastically scattered $v=2 \rightarrow 1$ and elastically scattered $v=2 \rightarrow 2$ will be extracted which will be referred to as vibration-to-translation transfer ( $\mathrm{V} \rightarrow \mathrm{T}$ transfer) in the further discussion.

The V-T transfer in the observed NO scattering from a wedge described above is shown in Figure 4.14: For clean $\mathrm{Au}(111)$, an energy increase of $39( \pm 4) \mathrm{meV}$ can be observed in the inelastic channel, a value in agreement with a previously reported value $(37( \pm 13) \mathrm{meV})$ for $\mathrm{NO}(v=3 \rightarrow 2)$ scattering for the same incidence energy. In proportion to the vibrational energy transferred $\left(\Delta \mathrm{E}_{\mathrm{vib}, \mathrm{NO}}=0.236 \mathrm{eV}\right)$, that is $17( \pm 2) \%$. In contrast, when $\mathrm{Ag}$ is present on the surface, qualitatively a decrease of $\mathrm{V}$ - T transfer is observed. Including only values above $3 \mathrm{ML} \mathrm{Ag}$, the translational energy increase is $17( \pm 5) \mathrm{meV}(7( \pm 2) \%$ of $\Delta \mathrm{E}_{\mathrm{vib}, \mathrm{No}}$ ). Those findings indicate that $\mathrm{V}$ - $\mathrm{T}$ coupling is exhibiting a small but measurable dependency on the kind of surface. In a previous study about scattering of $\mathrm{NO}(v=11)$ from Au and Ag, no significant difference in $\mathrm{V}-\mathrm{T}$ coupling between scattering from $\mathrm{Au}\left(11( \pm 3) \%\right.$ of $\left.\Delta \mathrm{E}_{\mathrm{vib}, \mathrm{No}}\right)$ and $\mathrm{Ag}(17( \pm 10) \%$ of $\Delta \mathrm{E}_{\mathrm{vib}, \mathrm{NO}}$ ) was found [48].

In contrast to NO with a decrease in the V-T transfer in Ag in comparison to $\mathrm{Au}$, an increase can be observed for $\mathrm{CO}$. Due to the low signal to noise ratio, the scatter in the data bigger, therefore a set of measurements of $\mathrm{CO}(v=2 \rightarrow 2,1)$ TOF measurements from a number of wedges will be included in this analysis. The translational energy increase due to vibrational energy loss is depicted in Figure 4.15. The data obtained from all wedges are showing the same trend: On Au(111), V-T transfer is absent, there is no difference in the TOF shapes from different vibrational channels (as seen in Figure 4.9, top panel). However, on $\mathrm{Ag}(111)$ a positive translational energy increase is obtained. A quantitative analysis yield $\mathrm{V}-\mathrm{T}$ transfer of $\mathrm{O}( \pm 3) \mathrm{meV}\left(\mathrm{O}( \pm 1) \%\right.$ of $\left.\Delta \mathrm{E}_{\text {vib,co }}=0.266 \mathrm{eV}\right)$ on $\mathrm{Au}(111)$ and of $16( \pm 3) \mathrm{meV}$ $\left(7( \pm 1) \%\right.$ of $\Delta \mathrm{E}_{\text {vib,co) }}$ on $\mathrm{Ag}(111)$ (from data of thicknesses $>3 \mathrm{ML} \mathrm{Ag} / \mathrm{Au}$ ). 


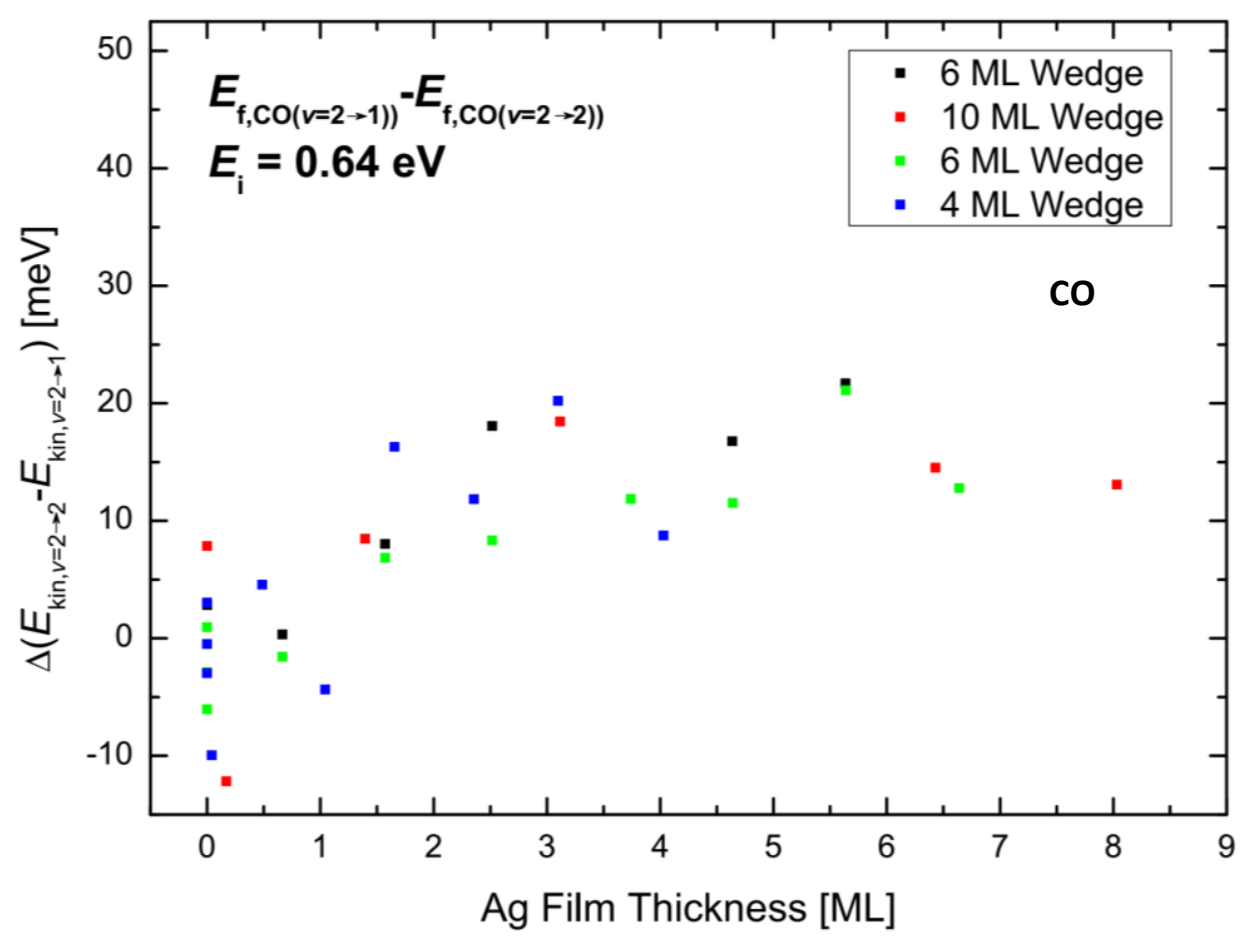

Figure 4.15: Translational energy increase of $\mathrm{CO}(v=2 \rightarrow 1)$ in comparison with $\mathrm{CO}(v=2 \rightarrow 2)$. Data obtained from a number of different wedges is shown. Note that the trend is the opposite direction as observed for NO (Figure 4.14).

Before starting the comparison of different surfaces, an emphasis should be set on the different behavior of $\mathrm{NO}$ and $\mathrm{CO}$ scattered off of $\mathrm{Au}(111)$. NO, similarly as $\mathrm{HCl}$, is known to exhibit a substantial increase of its recoil energy after vibrational relaxation. The absence of $\mathrm{V}-\mathrm{T}$ transfer leading to two indistinguishable TOF shapes for $\mathrm{CO}(v=2 \rightarrow 2)$ and $\mathrm{CO}(v=2 \rightarrow 1)$ is remarkable and has not been observed on any other molecule.

Concerning the mechanism of $\mathrm{V}-\mathrm{T}$ coupling, a number of possible hypothesis has been discussed in the context of $\mathrm{NO}(v=3 \rightarrow 2, v=3 \rightarrow 1)$. Generally, vibrational relaxation of $\mathrm{NO}$ is understood as an example of nonadiabatic coupling between the molecular vibration and electron hole pairs (EHPS). When NO is approaching the surface, its electron affinity level is energetically lowered until it accepts an electron to form a transient NO anion at a critical distance. After collision, the anion recoils until the neutral state is becoming favorable and the electron hops back to the surface. It was suggested that in $\mathrm{V} \rightarrow \mathrm{T}$ coupling, EHPs are playing a role as a mediator in energy transfer: First, the energy released after vibrational relaxation leads to the formation of hot electrons which can couple to translation. With higher incidence energy, it was envisioned that NO can penetrate into regions with higher electron density leading to stronger EHP-mediated V-T coupling.

Taking up this argumentation that stronger coupling to the surface electrons causes higher V-T transfer, the difference between $\mathrm{NO}$ and $\mathrm{CO}$ on $\mathrm{Au}(111)$ could be explained. Previous investigations on the strength of nonadiabatic interaction $\alpha$ of $\mathrm{NO}$ and $\mathrm{CO}$ with metal surfaces, including vibrational excitation during scattering from Au (NO [28], $\mathrm{CO}$ [29]) and interaction of hot electrons on NO/CO adsorbed on $\operatorname{Ir}(111)$ [90], proved that $\alpha(\mathrm{NO})>>\alpha(\mathrm{CO})$, easily understood by the difference of the electron affinity level $E_{\mathrm{A}}$. The affinity level of all molecules experiences the same image charge stabilization when approaching the surface and it might be assumed that the distance of closest approach is similar. Therefore, a molecule with a high tendency to accept electrons in the gas phase will undergo stronger nonadiabatic coupling as well. In the same line of thought, the observable V-T 
transfer of $\mathrm{CO}$ on $\mathrm{Ag}$ can be understood: Due to a lower work function of $\mathrm{Ag} / \mathrm{Au}$ in comparison to $\mathrm{Au}$, coupling might be facilitated. However, the same trend should be expected as for NO which is not the case. It seems obvious that there is not a single surface parameter that can generally describe this aspect of the scattering behavior for the two diatomic molecules. More experimental work will be necessary for a complete understanding of the different trends of VT-coupling for $\mathrm{CO}$ and NO.

\subsubsection{A Possible Mechanism Explaining the Film Thickness Trend}

The main result of the TOF experiments is a gradual change of the final mean translational energy within the formation of the first three monolayers of $\mathrm{Ag} / \mathrm{Au}(111)$. Which model can be employed to describe the observed trend?

In older work about molecular translational energy after scattering from a bulk surface, the already mention "Baule" model invoking single head-on collisions between molecule and a single surface atoms was successfully applied. Extending this idea to an ideal layer-by-layer growth thin film system like $\mathrm{Ag} / \mathrm{Au}$, one should expect that molecules colliding with an $\mathrm{Au}$ atom and those colliding with $\mathrm{Ag}$ atoms are found with the recoil energy according to the respective "Baule limit". Thus the final translational mean energy would change between thicknesses of zero and one monolayer when full coverage is reached and only Ag atoms can act as a collision partner. This simple model is not conform to the observation that the recoil energy is changing over a broader thickness range and is reaching a constant value at $3 \mathrm{ML} \mathrm{Ag} / \mathrm{Au}$. It is clear that at $1 \mathrm{ML} \mathrm{Ag} / \mathrm{Au}$, the mass of sublayer $\mathrm{Au}$ atoms still plays a role in the scattering process.

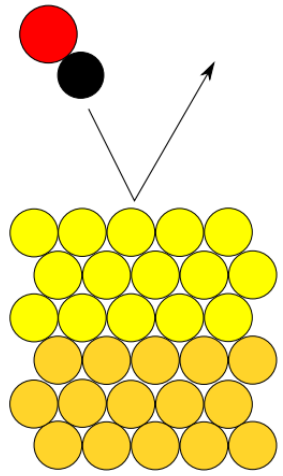

$\mathrm{Au}(111)$

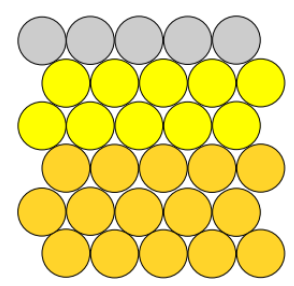

$1 \mathrm{ML} \mathrm{Ag/Au(111)}$

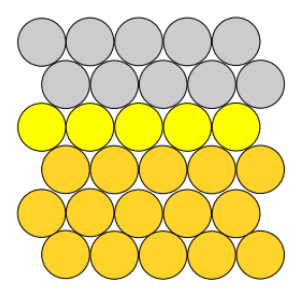

$2 \mathrm{ML} \mathrm{Ag} / \mathrm{Au}(111)$

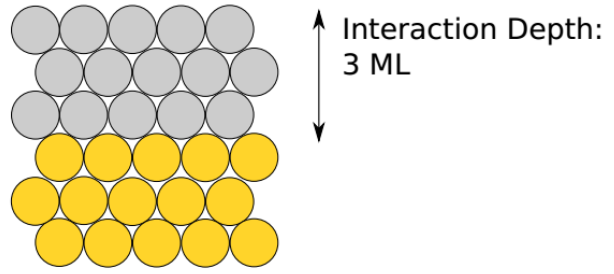

$3 \mathrm{ML} \mathrm{Ag/Au(111)}$

Figure 4.16: Interaction depth of a scattered molecule from Ag/Au. Shown is a cross-section of the surface in a side view. At a certain film thickness, the molecule "feels" an effective surface mass that can be approximated by averaging the masses of the atoms forming the first three monolayers (bright colors). Deeper layers (dark colors) are ignored in the translational energy transfer during the scattering event.

Implicitly the sublayer surface mass is accounted for when calculating the effective surface mass as in Figure 4.13. Instead of considering a specific surface atom where the collision occurs, one could imagine a rather abstract "interaction partner" of the molecule to which the obtained effective mass is assigned. Defining the interaction partner in a manner that agrees with the measurements, it is a good approximation to choose the topmost three monolayers. In fact, the linear trend of the effective mass change of 0-3 ML can be reproduced very well when invoking an average mass of those first three layers. This number can be interpreted as a kind of interaction depth, it tells us what kind of surface is "felt" by the molecule that hits a thin film surface as visualized in Figure 4.16. 
$\mathrm{Au}(111)$

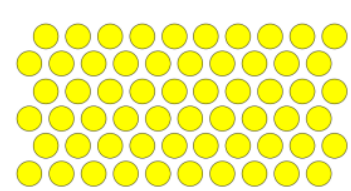

$1 \mathrm{ML} \mathrm{Ag} / \mathrm{Au}(111)$

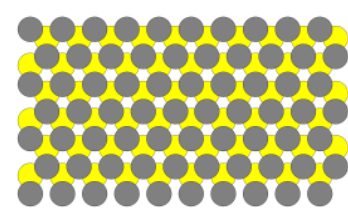

$2 \mathrm{ML} \mathrm{Ag} / \mathrm{Au}(111)$

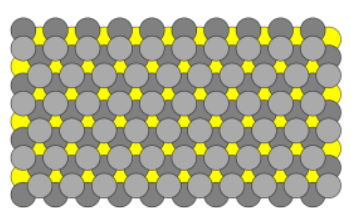

$3 \mathrm{ML} \mathrm{Ag} / \mathrm{Au}(111)$

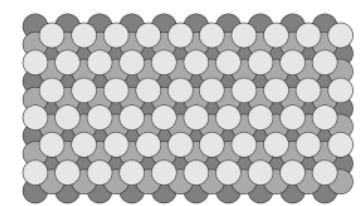

Figure 4.17: $\mathrm{Au}(111)$ surface shown at similar coverages from the perspective of an incoming molecule: With $3 \mathrm{ML} \mathrm{Ag/Au,} \mathrm{no}$ (hollow) sites with Au are visible anymore, the $A B C$ stacking of $A g$ is completed.

In the concrete example of $\mathrm{Ag} / \mathrm{Au}$, the number three might be characteristic for the structural properties of the surface: Noble metals like $\mathrm{Au}$ and $\mathrm{Ag}$ are constructed as a fcc lattice and the epitaxial growth of silver on top of the $A u(111)$ surface means continuing this lattice of a close-packing of equal spheres. With the third monolayer, a complete $A B C$ stacking of $\mathrm{Ag}$ is formed and for an incoming molecule, sites with Au are not visible anymore (Figure 4.17). 


\section{Vibrational Inelasticity of $\mathrm{NO}(v=2)$ after Scattering from $\mathrm{Ag} / \mathrm{Au}(111)$}

Vibrational energy transfer in gas-surface scattering gained increasing interest in recent years when it was shown that the electronic states of the surface play an important role in the interaction with molecular vibrational states. In this context, one key experiment was the scattering of vibrationally excited NO scattered from LiF and $\mathrm{Au}(111)$ [43]. While almost no vibrational relaxation was observed when using LiF as a scattering target, a broad distribution of vibrational states was observed after scattering from a $\mathrm{Au}(111)$ surface.

This section describes how absolute vibrational relaxation probabilities of excited diatomic molecules are obtained after scattering from $\mathrm{Ag} / \mathrm{Au}(111)$ surfaces with defined thickness. The measurement strategy focuses on the comparison of REMPI signals from different thin film surfaces to the reference surface of $\mathrm{Au}(111)$ providing a method to determine relative relaxation probabilities in a quantitative manner. Finally with the knowledge of absolute scattering probabilities on the reference surface, this measure can be obtained for the thin film systems as well.

The evaluation of vibrational relaxation probabilities follow the previously reported method by Cooper et al. [28]. For both $\mathrm{NO}$ and $\mathrm{CO}$ which were investigated in this work, molecules were prepared in initial vibrational state $v=2$. After scattering from the surface, molecules can stay in the same $(v=2)$ or relax into a lower vibrational state $(v=1,0)$. With a surface at room temperature, the vibrational excitation probability to $v=3$ is $<<1 \%$ [41] and can therefore be neglected. First, the relaxation probability $P_{v=2-x}$ of diatomic molecules in the incident vibrational state $v_{\mathrm{i}}=2$ that relax into final state $v_{f}=\mathrm{x}$ can be defined by the ratio of scattered molecules $N_{\mathrm{x}}$ to the sum scattered molecules $\sum_{i} N_{v=i}$ involved in the relaxation process.

$$
P_{v=2 \rightarrow x}=\frac{N_{v=x}}{\sum_{i} N_{v=i}}
$$

Ideally, that would also include molecules in the vibrational ground state $v=0$. However, due to a strong background of molecules from the incoming beam which have not been excited to $v=2$, that state is experimentally not accessible. Using the correct terminology, only "branching ratios" between molecules in the states $v=2,1$ can obtained. Nevertheless, by expecting only weak relaxation behavior at a $\mathrm{Au}(111)$ surface, an assumption which will be discussed in the section about error estimation, the relaxation into $v=0$ is neglected, leading directly to a scattering probability only by comparing $v=2,1$.

Quantitative information about the number of molecules $N_{v=x}$ in a vibrational state can be obtained from the integrated spectral intensity $\int S(\lambda) d \lambda$. Those measurements are strongly affected by fluctuations of the laser power which was continuously monitored allowing a point-to-point correction as elaborated in the experimental section. Furthermore, integrated area depends on several factors like changes of the angular distribution $\Theta_{v}(\theta)$, the arrival time distribution $\Delta\left(\tau_{v}\right)$ and the FranckCondon-Factors $F C F_{v}$ of the corresponding vibrational band. To minimize corrections, the detector gain $\Gamma_{v}$ defined by the voltage of the MCP detector was kept constant. Because acquisition via REMPI is a density-dependent detection technique, density-to-flux conversion is carried out by multiplying with the scattered mean velocity $\left\langle s_{v}\right\rangle$. All corrections included, the intensity of different vibrational states can be quantitatively compared: 


$$
N_{v} \propto\left\langle s_{v}\right\rangle \cdot \Theta_{v} \cdot \Delta\left(\tau_{v}\right) \cdot F C F_{v}^{-1} \cdot \Gamma_{v} \cdot \int S_{v}(\lambda) d \lambda
$$

The outlined procedure is employed to obtain absolute scattering probabilities $P_{v=2 \rightarrow 2}(\mathrm{Au})$ and $P_{v=2 \rightarrow 1}(\mathrm{Au})$ from a Au(111) surface for both molecules $\mathrm{NO}(v=2)$ and $\mathrm{CO}(v=2)$.

For the comparison of relaxation at a silver film surface with thickness of $T$ monolayers $\mathrm{Ag} / \mathrm{Au}$ to a reference surface $A u(111)$, a relative relaxation probability $P_{r e l, v=2 \rightarrow x}$ into final state $v_{f}=x$ is introduced. In combination with equation (5.1), it is obvious that this measure can be expressed by the number of molecules detected after scattering from the compared surfaces:

$$
P_{r e l, v=2 \rightarrow x}(T \mathrm{ML} \mathrm{Ag} / \mathrm{Au})=\frac{P_{v=2 \rightarrow x}(T \mathrm{ML})}{P_{v=2 \rightarrow x}(\mathrm{Au})}=\frac{N_{v=x}(T \mathrm{ML})}{N_{v=x}(\mathrm{Au})}
$$

For a single vibrational state and using a constant detector gain $\Gamma_{v}, N_{v}$ can be substituted according to equation (5.2).

$$
P_{r e l, v=2 \rightarrow x}(T \mathrm{ML} \mathrm{Ag} / \mathrm{Au})=\frac{\left\langle s_{v=x}\right\rangle(T \mathrm{ML})}{\left\langle s_{v=x}\right\rangle(\mathrm{Au})} \cdot \frac{\Theta_{v=x}(T \mathrm{ML})}{\Theta_{v=x}(\mathrm{Au})} \cdot \frac{\Delta\left(\tau_{v=x}, T \mathrm{ML}\right)}{\Delta\left(\tau_{v=x}, \mathrm{Au}\right)} \cdot \frac{\int S_{v=x}(\lambda, T \mathrm{ML}) d \lambda}{\int S_{v=x}(\lambda, \mathrm{Au}) d \lambda}
$$

Introducing a few abbreviations for the relative signal and the three correction factors

$$
S I G_{r e l, x}=\frac{\int S(\lambda, T \mathrm{ML}) d \lambda}{\int S(\lambda, \mathrm{Au}) d \lambda}, C F_{A n g, x}=\frac{\Theta_{v=x}(T \mathrm{ML})}{\Theta_{v=x}(\mathrm{Au})}, C F_{T O F, x}=\frac{\Delta\left(\tau_{v=x}, T \mathrm{ML}\right)}{\Delta\left(\tau_{v=x}, \mathrm{Au}\right)}, C F_{D T F, x}=\frac{\left\langle s_{v=x}\right\rangle(T \mathrm{ML})}{\left\langle s_{v=x}\right\rangle(\mathrm{Au})}
$$

in accordance with (5.3), the absolute scattering probability of a diatomic molecule scattered from a surface of $T$ monolayer thickness can be obtained as

$$
P_{v=2 \rightarrow x}(T \mathrm{ML})=P_{v=2 \rightarrow x}(\mathrm{Au}) \cdot S I G_{r e l, x} \cdot C F_{A n g, x} \cdot C F_{T O F, x} \cdot C F_{D T F, x}
$$

which allows to obtain the absolute relaxation probabilities of the observed final states $v=2,1$. Although we assume no relaxation into the vibrational ground state $v=0$ for the reference surface $\mathrm{Au}(111)$, on silver films it might be possible to observe a stronger relaxation behavior allowing relaxation into this state.

\section{$5.1 \quad$ Results}

This section describes the measurement of signal intensities obtained by the state-selective ionization (REMPI) of molecules that scatter from a surface with a defined film thickness. Because the signal measured at a $\mathrm{Au}(111)$ surface is always employed as an internal standard, the short expression "REMPI signal" will refer to the relative signal intensity $S I G_{r e l}$ in comparison to the internal Au(111) standard.

Two approaches are chosen to acquire the REMPI signal intensity of NO scattered from thin silver films which yield the same result: The first method is based on the detection of a REMPI signal for a single rotational state of $\mathrm{NO}$ scattered from a wedge of $\mathrm{Ag} / \mathrm{Au}(111)$. The REMPI laser is set to a wavelength resonantly ionizing a part of the broad rotational distribution within a vibrational state. The signal from this rotational state is then regarded as representative for the spectrum of the corresponding vibrational state. The second method takes the full spectrum of a vibrational state into account. Here, 
instead of a wedge, the scattering target is a homogeneous film with defined thickness covering half of the $\mathrm{Au}(111)$ crystal. Both approaches lead to the same results as will be shown in the following sections. In all measurements, the signal obtained from a $A u(111)$ surface is used as a reference, therefore all signals will be normalized to this value.

\subsubsection{Acquisition of $\mathrm{NO}(v=2 \rightarrow 2)$ REMPI signals}

The REMPI signals $S I G_{r e l}$ of the $\mathrm{NO}(v=2 \rightarrow 2)$ channel scattered from Au and a thin film wedge sample were measured and plotted against the assigned film thickness. Figure 5.1 shows the data obtained with the same gas mixture (incidence energy $E_{\mathrm{i}}=0.59 \mathrm{eV}$ ) from a number of samples. Let us first concentrate on the data sets which were acquired from a selected rotational state of the vibrational spectrum: From a signal measurement along a clean $\mathrm{Au}(111)$ crystal (see Figure 3.26, right panel), the error of a signal measurement at a certain position is estimated to be $5 \%$ due to laser fluctuations. Although only a statistical error, it might lead to small systematic shifts because the measurements defining the common reference signal are affected as well. All data sets display high consistency independent of the thickness gradient or the chosen rotational state.

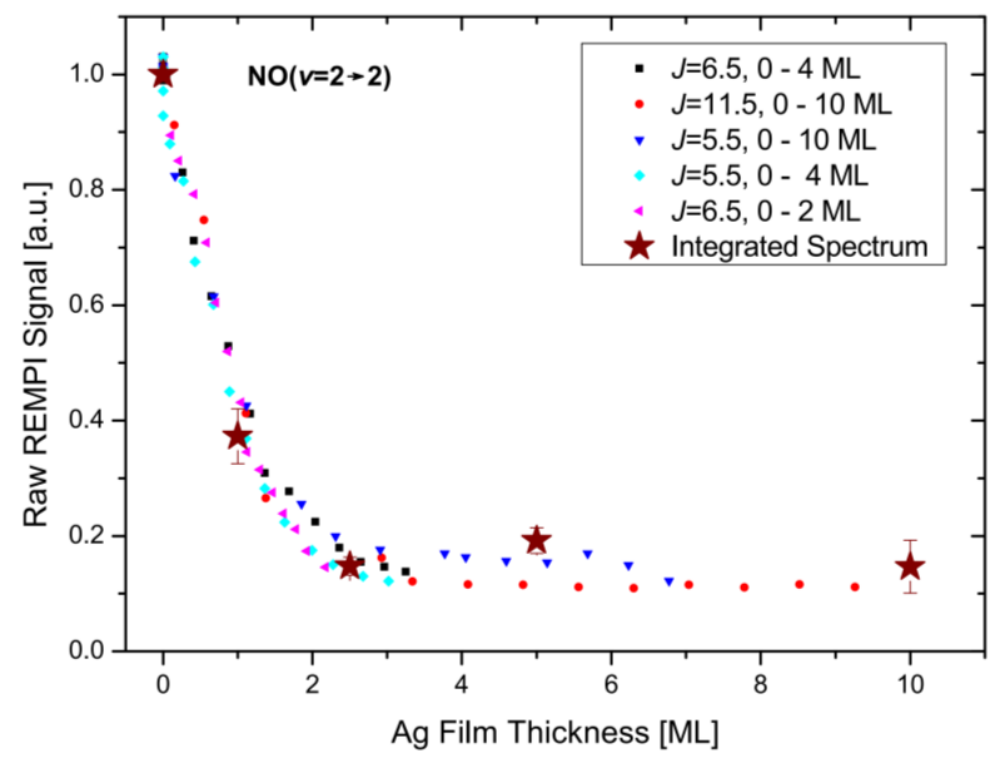

Figure 5.1: Raw REMPI signals of the $\mathrm{NO}(v=2 \rightarrow 2)$ scattering channel for different silver film thicknesses. As a common reference, the signal observed at $\mathrm{Au}(111)$ is set to one.

For the first 0-3 ML Ag/Au(111), it is observed that the signal is decreasing to $10-15 \%$ of the signal on $\mathrm{Au}(111)$ which is a measure for the survival probability decreasing with $\mathrm{Ag}$ on the surface. From three monolayer thickness on, a constant signal intensity is observed. Moreover, the plot Figure 5.1 includes data obtained with a second measurement strategy. Instead a single rotational line, the full spectrum of the vibrational spectrum is recorded. The results obtained from different methods is very consistent. A reason for this good agreement can be found by comparing the NO spectra for different surfaces. One representative example, the spectrum of the $\mathrm{NO}(v=2 \rightarrow 2)$ channel obtained after scattering from $\mathrm{Au}(111)$ and $10 \mathrm{ML} \mathrm{Ag} / \mathrm{Au}$, is depicted in Figure 5.2. In the bottom panel, the spectrum which obtained from scattering off a silver film is multiplied by a constant factor of 5.9, resulting in two matching spectra. In the context of energy transfer on surfaces this means that the rotational distribution of 
scattered NO does not change when the surface is changed from Au to a silver film. If that is the case, the intensity of a single rotational state provides the information of the full spectrum which allows to facilitate the experiment. As a consequence, both methods to record the REMPI signals are equally adequate with the difference that the measurement of a single rotational state is less prone to errors than measuring a full spectrum.

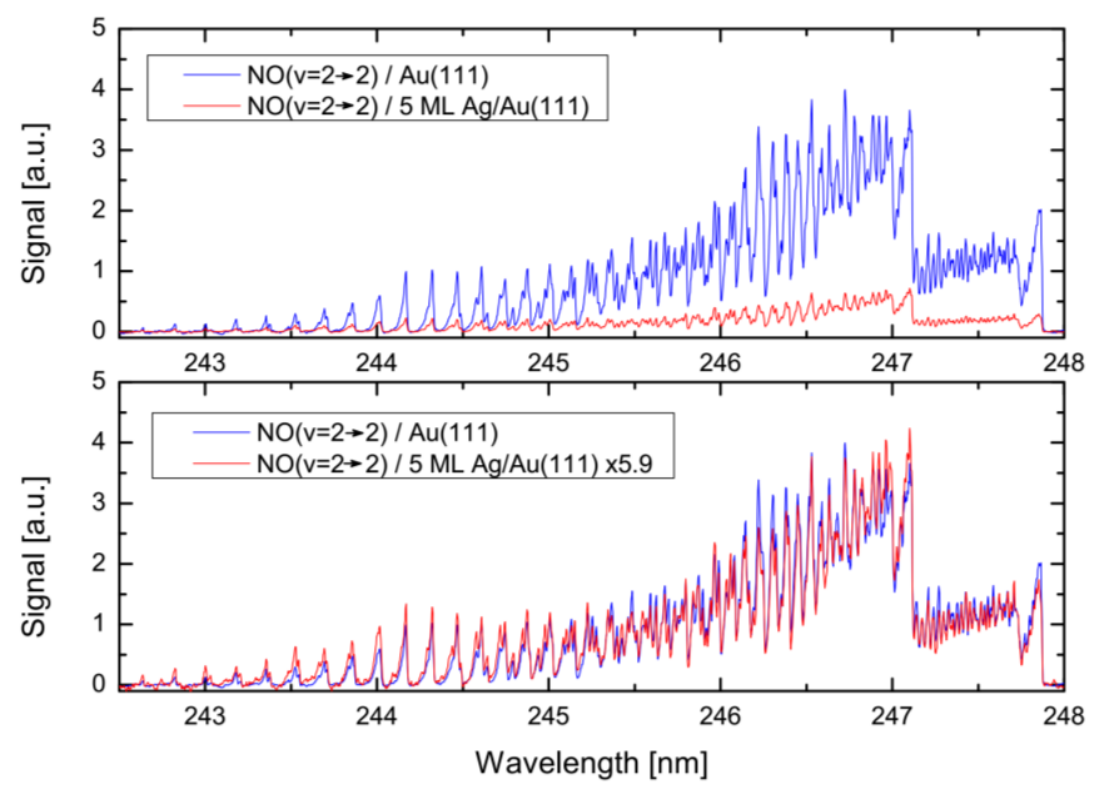

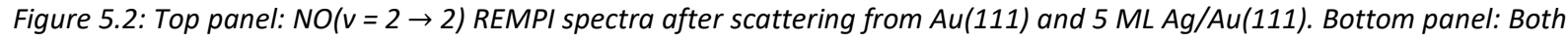
spectra match when scaling the NO/Ag spectrum by a constant factor.

\subsubsection{Acquisition of $\mathrm{NO}(v=2 \rightarrow 1)$ REMPI signals}

While measurement of the $\mathrm{NO}(v=2 \rightarrow 2)$ scattering channel are highly consistent on different samples, the same measurement strategy proofed difficult for the inelastic scattering channel $\mathrm{NO}(v=2 \rightarrow 1)$.
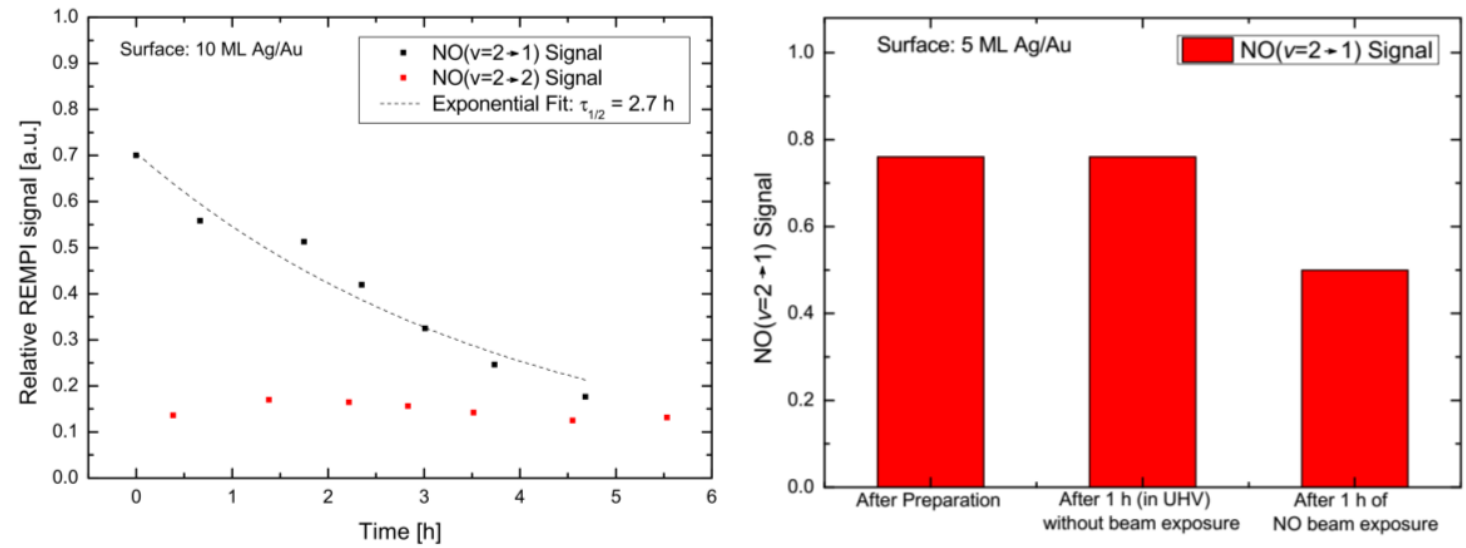

Figure 5.3: REMPI signals over time. Left panel: While the relative $N O(v=2 \rightarrow 2)$ signal is constant over time, significant decrease can be observed in the $\mathrm{NO}(v=2 \rightarrow 1)$ channel. Right panel: The observed signal decreased is due to the exposure to the molecular beam with the $\mathrm{NO} / \mathrm{H}_{2}$ gas mixture. 
When measuring its signal over time, it was observed that the $\mathrm{NO}(v=2 \rightarrow 1)$ scattering signal intensity displayed a decrease measured on a silver film surface. As a result, the relative signal (referenced to the $\mathrm{NO}(v=2 \rightarrow 1)$ signal on $\mathrm{Au}(111))$ is decreasing over time (Figure 5.3, left panel).

Further investigation showed that this signal decrease is due to the exposure to the molecular beam. On a film of $5 \mathrm{ML} \mathrm{Ag} / \mathrm{Au}$, the $\mathrm{NO}(v=2 \rightarrow 1)$ signal relative to the signal obtained on $\mathrm{Au}(111)$ remained constant when the sample was kept in UHV. However, after one hour of exposure to the molecular beam, a signal decrease was detected. AES measurements did not show any signs of nitrogen or oxygen on the surface which assumes that only very subtle changes on the surface cause this effect. As a consequence, for the rest of discussion, it will only concentrated on the $\mathrm{NO}(v=2 \rightarrow 1)$ signal directly after the surface was prepared, referred to as "clean surface limit".

For this "clean surface limit", the REMPI signal of $\mathrm{NO}(v=2 \rightarrow 1)$ at different Ag film thicknesses is depicted in Figure 5.4. Here, the signal intensity of a single rotational state was selected. For the bulk limit of $3 \mathrm{ML} \mathrm{Ag} / \mathrm{Au}$, the REMPI intensity is with $70 \%$ substantially higher than the $\mathrm{NO}(v=2 \rightarrow 2)$ signal (compare to Figure 5.1).

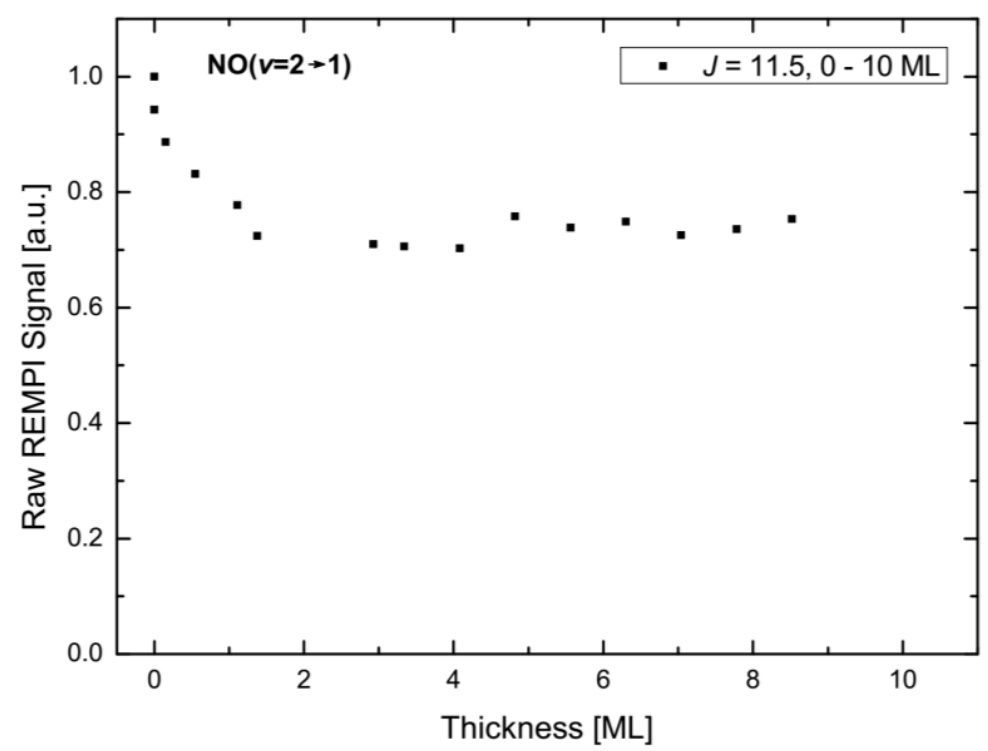

Figure 5.4: Raw REMPI signals of the NO(v=2 $\rightarrow 1)$ scattering channel for different silver film thicknesses. As a common reference, the signal observed at $A u(111)$ is set to one.

Again, it was confirmed that a single rotational state can be regarded as a representative for the full spectrum. Figure 5.5 (top panel) shows the two $\mathrm{NO}(v=2 \rightarrow 1)$ spectra obtained from Au and $5 \mathrm{ML}$ $\mathrm{Ag} / \mathrm{Au}$. When scaling the spectrum obtained at $5 \mathrm{ML} \mathrm{Ag} / \mathrm{Au}$ by a constant factor (bottom panel), both spectra from different surfaces agree very well within the error of the experiment. 

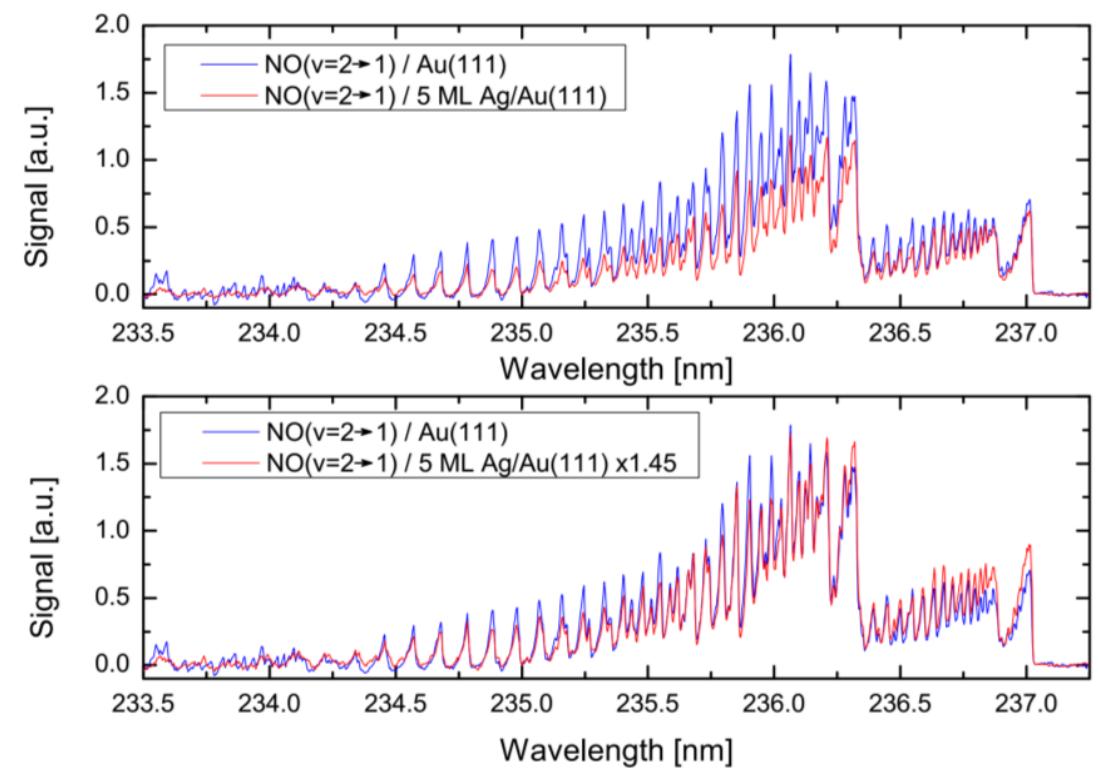

Figure 5.5: Top panel: $N O(v=2 \rightarrow 1)$ REMPI spectra after scattering from $A u(111)$ and $5 \mathrm{ML} A g / A u(111)$. Bottom panel: Both spectra match when scaling the NO/Ag spectrum by a constant factor.

\subsubsection{Corrections for Quantitative Analysis}

Acquiring signal intensities for different surfaces is done by REMPI ionization via a focused UV laser. At the set REMPI delay the laser spot ionizes only a small fraction of the scattered molecules distribution in time and space. Any changes of that distribution will influence the obtained signal intensity. The characteristics which are changing from gold to silver films are the angular distribution, the TOF shape and the final velocity of the molecules. Suitable correction factors $C F_{A n g}, C F_{T O F}$ can be obtained from experimental data. The detection via REMPI ionization is a density-dependent measurement, therefore a density-to-flux conversion will be carried out by applying the correction factor $C F_{D T F}$.

\section{Angular Distribution}

For both vibrational channels $\mathrm{NO}(v=2 \rightarrow 2,1)$, the angular distributions were recorded for $\mathrm{Au}(111)$, $1 \mathrm{ML}$ and $3 \mathrm{ML} \mathrm{Ag} / \mathrm{Au}$ film thickness. Data and fits according to a $\operatorname{Cos}\left[\theta-\theta_{0}\right]^{n}$ function $\left(\theta_{0}\right.$ : reflecting angle) are depicted in Figure 5.6. In all cases, we gain narrow distributions characterized by high values for exponent $n(>20)$ which are typical for a direct-scattering mechanism described for NO scattered off $\mathrm{Au}[28,43]$ and $\mathrm{Ag}[6]$. In the special case of thin films, narrow angular distributions give additional indication for a flat, non-corrugated surface. A slight broadening was detected in the transition from $\mathrm{Au}$ to $\mathrm{Ag} / \mathrm{Au}$ in the case of vibrationally elastic scattering $\mathrm{NO}(v=2 \rightarrow 2)$. A similar effect can be found when comparing the angular distributions of $\mathrm{NO}(v=0 \rightarrow 0)$ scattering from bulk samples of $\mathrm{Au}(111)$ and $\mathrm{Ag}(111)$ (see Appendix C). 

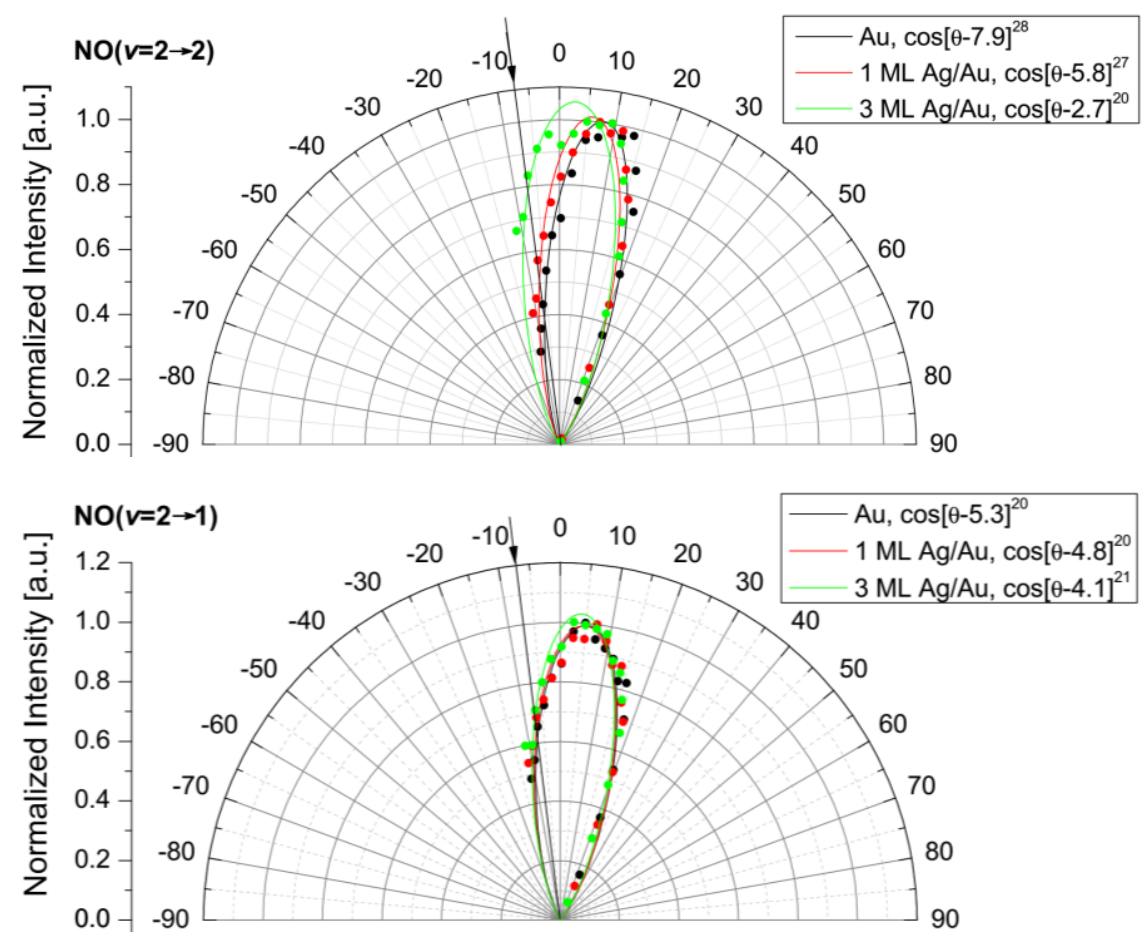

Figure 5.6: Angular Distributions of $\mathrm{NO}(v=2 \rightarrow 2)$ (top panel) and $\mathrm{NO}(v=2 \rightarrow 1)$ (bottom panel) for three different surfaces. The incoming angle (ca. $\left.7^{\circ}\right)$ is marked by a small arrow.

For the following procedure, the angular distribution were fitted with a Gaussian function $f(\theta)=$ $\operatorname{Exp}\left(\frac{\left(\theta-\theta_{0}\right)^{2}}{2 \sigma^{2}}\right)$ to yield the fit parameter $\sigma$ which tells about the FWHM of the distribution (Figure 5.7). Correction factors $C F_{A n g}$ were obtained after normalization to the value measured on $\mathrm{Au}(111)$, values in between were assumed to follow a linear trend. That might seem to be a very crude approximation, however, the influence due to the change of the angular distribution is rather small. Due to negligible change in the $\mathrm{NO}(v=2 \rightarrow 1)$ angular distribution, no correction factor is employed. The results are plotted in Figure 5.7.
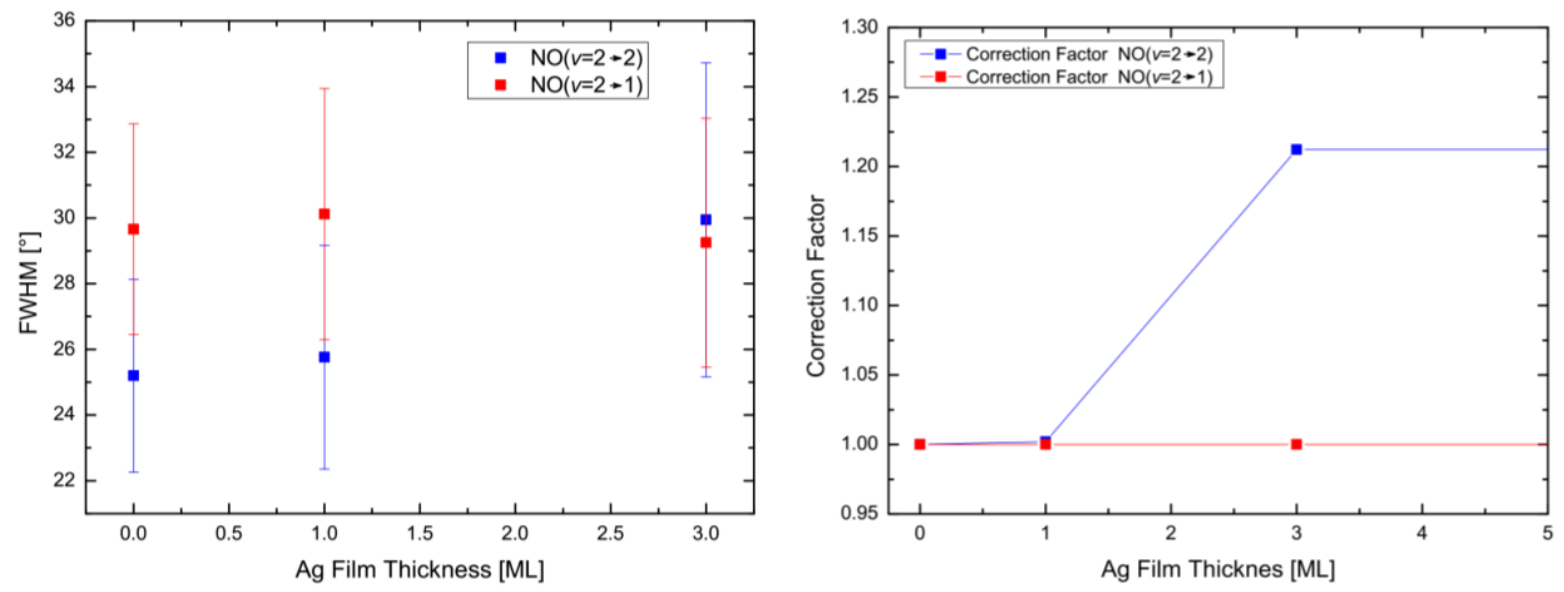

Figure 5.7: Left panel: FWHM obtained from a Gaussian fit. Right panel: Angular distribution correction factors for $N O(v=2 \rightarrow 2,1)$ at different film thicknesses.

The strongest correction will be performed in the case for vibrationally elastic scattering whereas the inelastic scattering channel correction is rather negligible. For a quantitative analysis, REMPI signals 
described in the previous section need to be multiplied by the depicted factor to account for the broadening of the angular distributions.

\section{Arrival Time Distributions (TOF)}

Measuring the arrival time distributions as discussed in the previous chapter (see section 4.1.1), it becomes clear that measurements from a silver-covered surface exhibit a broader TOF distribution in comparison to clean $\mathrm{Au}(111)$. A correction $C F_{T O F}$ can be gained via integration over the distribution at different thicknesses. The value obtained from clean $\mathrm{Au}(111)$ was used as a reference, accordingly the correction factor on $\mathrm{Au}(111)$ is 1.0. The results are depicted in Figure 5.8. Due to the broadening, we observe an increase of the correction factor from 1 to about 1.8 for $\mathrm{NO}(v=2 \rightarrow 2)$, respectively 1.7 for $\mathrm{NO}(v=2 \rightarrow 1)$. For film thicknesses above $3 \mathrm{ML}$, a constant factor was assumed as marked in the figure. In comparison to the other correction factors, this aspect represents strongest influence on the raw REMPI signal data.

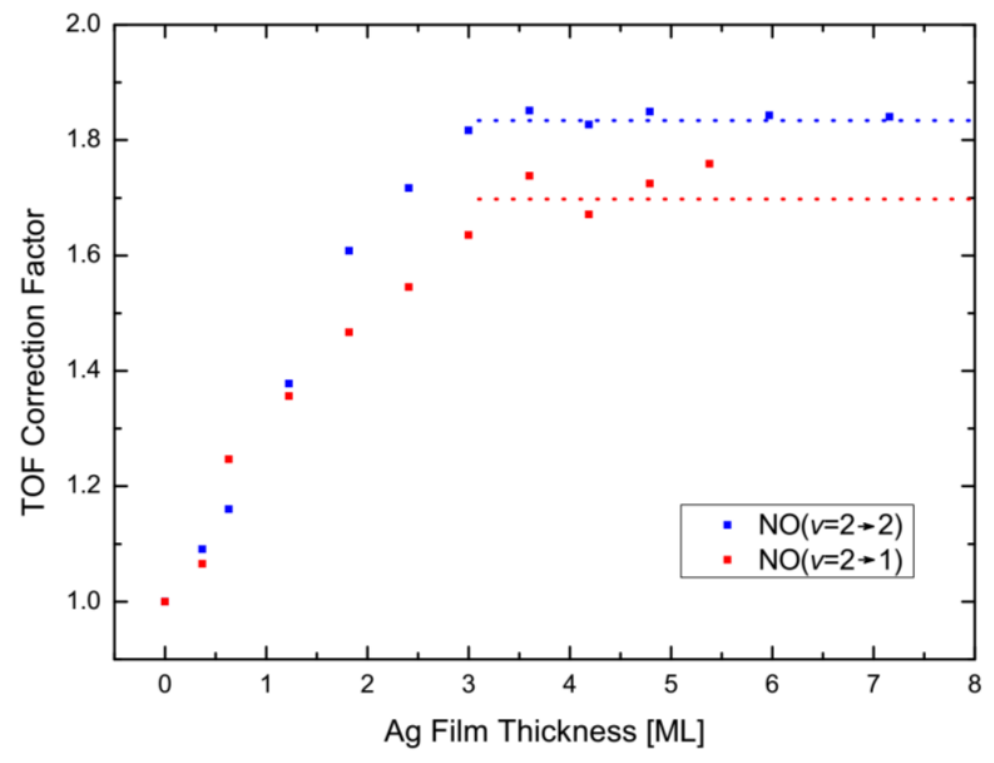

Figure 5.8: Correction factors due to the broadening of the arrival time distribution.

\section{Density-to-Flux conversion}

A final correction needs to be applied due to the detection process: The REMPI signals are proportional to the density of the scattered molecules, therefore the velocity of the scattered molecules influences the detected intensity [82]. Translational energies have already been obtained during the TOF experiments on different film thicknesses (see chapter 4). A correction factor can be calculated with the average velocity of the scattered molecules for a certain film thickness. When $A u(111)$ is again chosen as a reference, correction factors are obtained as shown in Figure 5.9. The data is showing a trend opposite to the TOF correction factors and compensates their effect to a certain degree. 


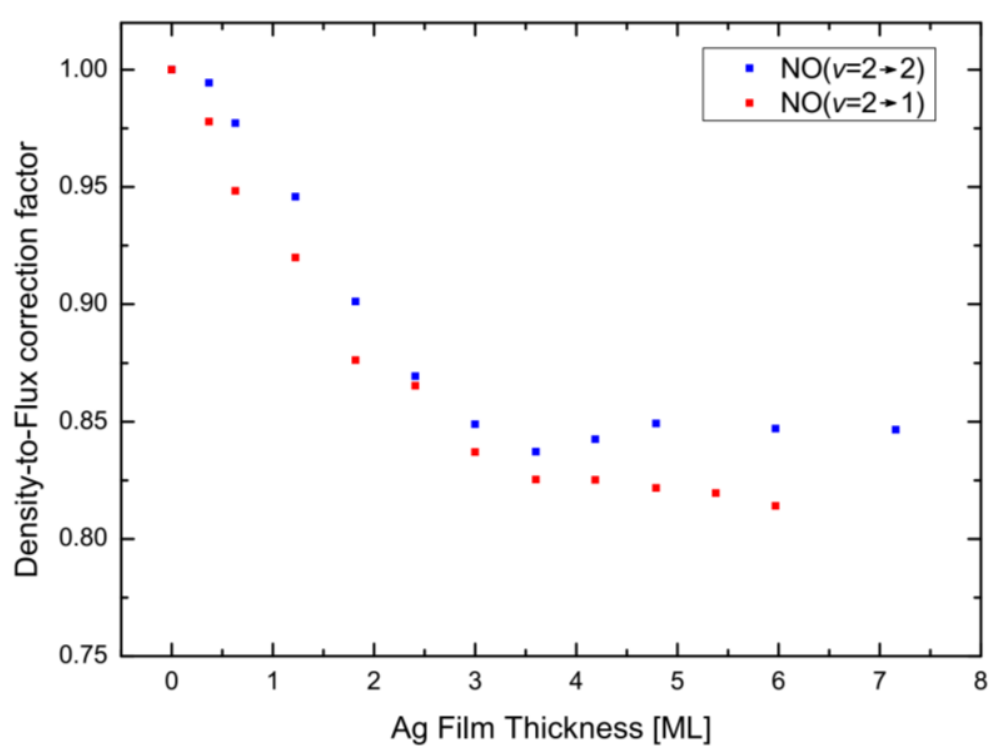

Figure 5.9: Correction factors for the density-to-flux conversion.

\section{Application of the correction factors}
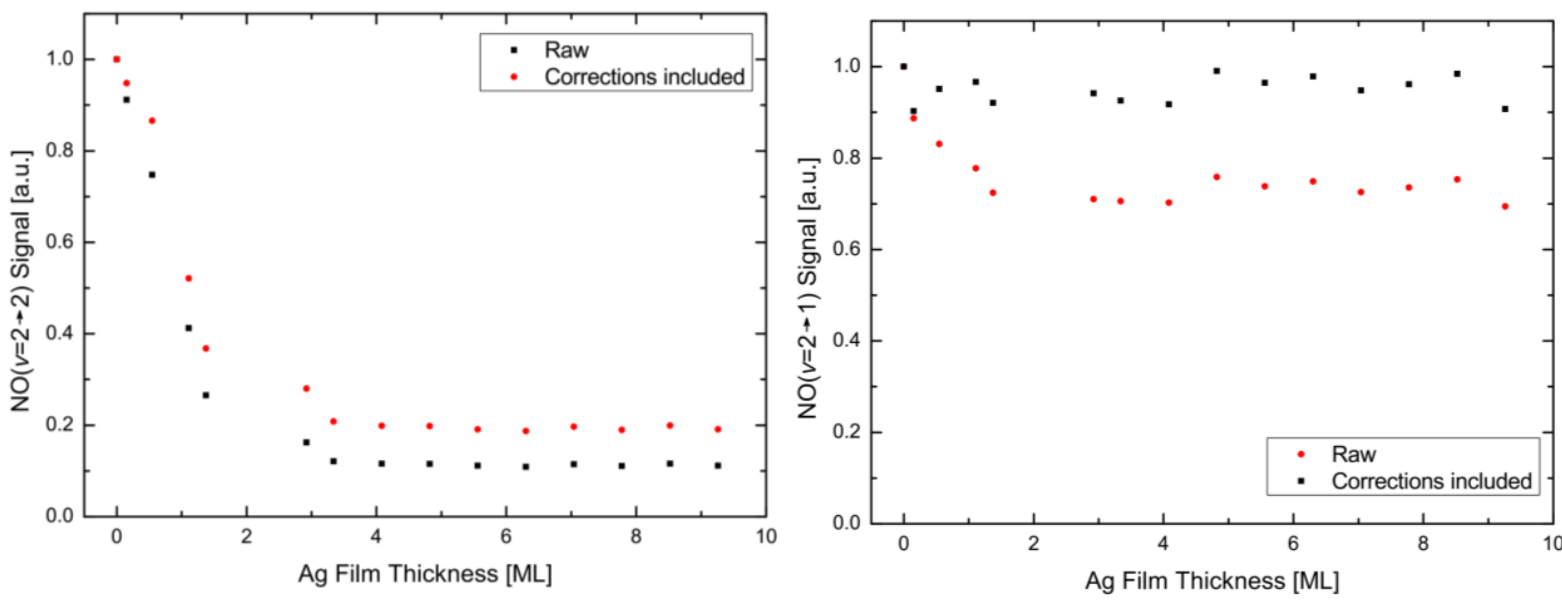

Figure 5.10: REMPI signals with and without including the discussed correction factors. Left panel: NO( $v=2 \rightarrow 2)$ scattering channel. Right panel: $\mathrm{NO}(v=2 \rightarrow 1)$ scattering channel.

Figure 5.10 shows the raw REMPI signals and the signals after application of the previously discussed correction factors. It is clear that quantitatively the overall picture is conserved. The most interesting result is probably that the thickness-dependent decrease observed in the $\mathrm{NO}(v=2 \rightarrow 1)$ signal can solely be explained by the broadening of the arrival time distributions. Relaxation into $\mathrm{NO}(v=2 \rightarrow 1)$ seems to be independent of the film thickness (at least for the regarded case of a freshly prepared surface). 


\subsubsection{Determination of Absolute Relaxation Probabilities}

\subsubsection{Absolute Relaxation Probabilities on Au(111)}

Relaxation probabilities relative to a $\mathrm{Au}(111)$ can be easily converted with the information of the absolute relaxation probabilities from that surface. For $\mathrm{NO}(v=2)$, this information was obtained in a previous study [45]. For the employed incidence energy of $\sim 0.6 \mathrm{eV}$, the survival probability was reported as $70( \pm 20) \%$, neglecting the relaxation into $v=0$. A number of REMPI spectra (Figure 5.11) measured for $\mathrm{NO}(v=2 \rightarrow 2)$ and $\mathrm{NO}(v=2 \rightarrow 1)$ from $\mathrm{Au}(111)$ was evaluated to confirm the reported value according to the procedure described in the introduction of this chapter (eq. 5.1 \& 5.2).

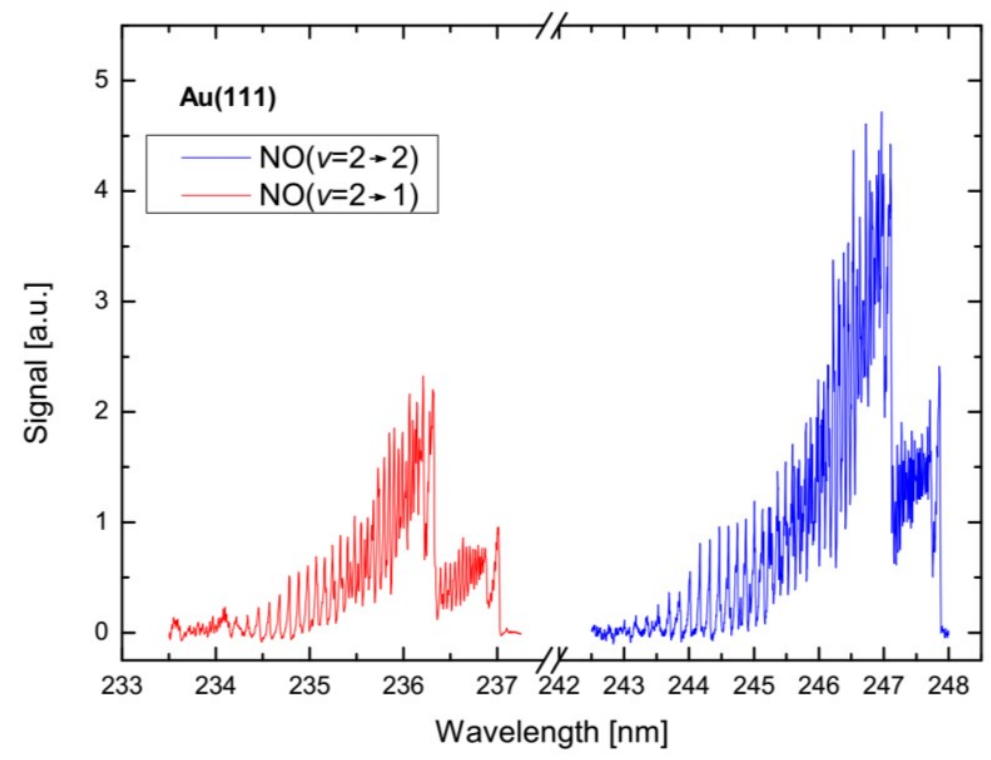

Figure 5.11: Spectra of $\mathrm{NO}(v=2 \rightarrow 2)$ and $\mathrm{NO}(v=2 \rightarrow 1)$ after scattering from $\mathrm{Au}(111)$.

Due to a high number of scans and the use of a point-to-point laser power correction, the biggest error source, the fluctuation of the laser could be minimized. Influences of angular distribution, width of the TOF distribution and the velocity were taken account of for every vibrational state similarly as outlines in the previous sections. Vibrational state dependent Franck-Condon factors were included in the analysis [91] but are similar ( $\left.F C F_{v=2}: 0.23793 ; F C F_{v=1} 0.26393\right)$ having only little effect on the analysis.

The survival probability for $\mathrm{NO}(v=2)$ was determined to be $74( \pm 3) \%$, the remaining $26( \pm 3) \%$ relax into NO in $v=1$. Note that the low

\subsubsection{Absolute Relaxation Probabilities of Ag/Au films}

After applying the conversion procedure, absolute relaxation probabilities for the two channels $\mathrm{NO}(v=2 \rightarrow 2,1)$ can be directly obtained. Both channel are depicted in the top and central panel of Figure 5.12 . For $\mathrm{NO}(v=2 \rightarrow 2)$, the survival probability, a strong decrease from initially $74 \%$ to $14 \%$ is observed. The lowest value of $14 \%$ is reached at a thickness of 3-4 ML Ag/Au which does not change even for higher film thicknesses. A low survival probability means that a higher amount of molecules loses its vibrational energy when colliding with a silver-coated surface. 


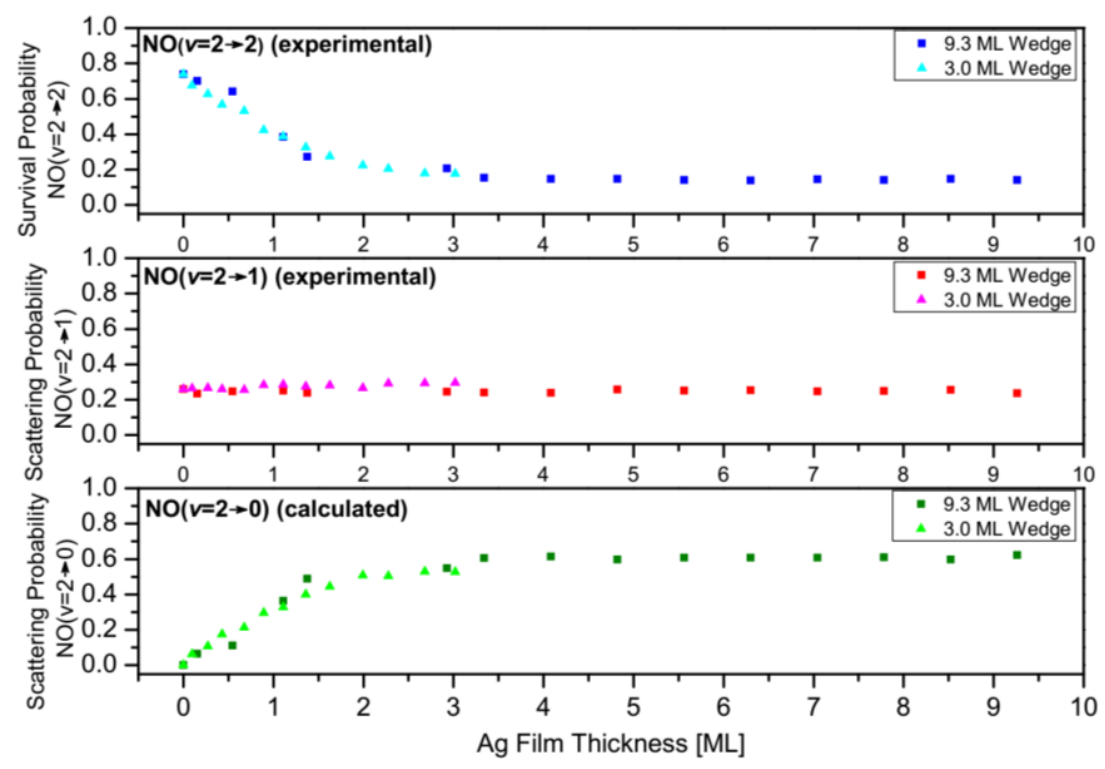

Figure 5.12: Absolute relaxation probabilities of $\mathrm{NO}(v=2)$ into lower vibrational states. Top panel: Probability to remain in the state $v=2$, also termed as "survival probability", obtained from experimental data. Central panel: Probability to relax into $v=1$, obtained from experimental data. Bottom panel: Probability to relax into $v=0$, by assuming that all remaining molecules not found in $v=2,1$ underwent relaxation into the ground state.

In which vibrational state are the molecule found after relaxation? The probability to relax into $v=1$ is constant over the full range of investigated film thicknesses and does not compensate the loss in $v=2$. The remaining possibility is the relaxation channel $\mathrm{NO}(v=2 \rightarrow 0)$ because excitation into higher vibrational states can be excluded at a room temperature surface. From the directly obtained absolute relaxation channels, the relaxation probability into $v=0$ is calculated and depicted in the bottom panel of Figure 5.12. We see that the relaxation probability into that channel does increase from $0 \%$ on $\mathrm{Au}(111)$ to $60 \%$ on silver films.

From the results in Figure 5.12, it is obvious that discussing about relaxation can be simplified by concentrating on a single measure, the survival probability $P_{\text {surv. }}$. Because it can induce confusion using that phrase to indicate the amount of relaxation, a new term, the relaxation probability $P_{\text {Relax }}=\left(1-P_{\text {Surv }}\right)$ will be introduced and used in the following discussion. Using that new term, $P_{\text {Relax }}$ increases for 0 $3 \mathrm{ML} \mathrm{Ag} / \mathrm{Au}$ from $26 \%$ to $86 \%$.

\subsubsection{Error Estimation}

In laser-based experiments, the biggest errors are typically introduced by laser power fluctuations (see error bars of Fig. 2 in [45]). In this work, great care has been taken to minimize those errors by monitoring the laser power. Due to a point-to-point laser power correction, a high number of scans (46 per vibrational state) measured on different days, the error bar due to statistical errors could be minimized.

Using the pure $\mathrm{Au}(111)$ surface for the measurement of REMPI signal intensities for a rotational state is a good example to estimate the reproducibility error (Figure 3.26, right panel). Only small deviations from a flat top are present in the data when the center of the crystal is scanned with a standard deviation of $3 \%$ which defines the relative error of the signal intensity measurement. 


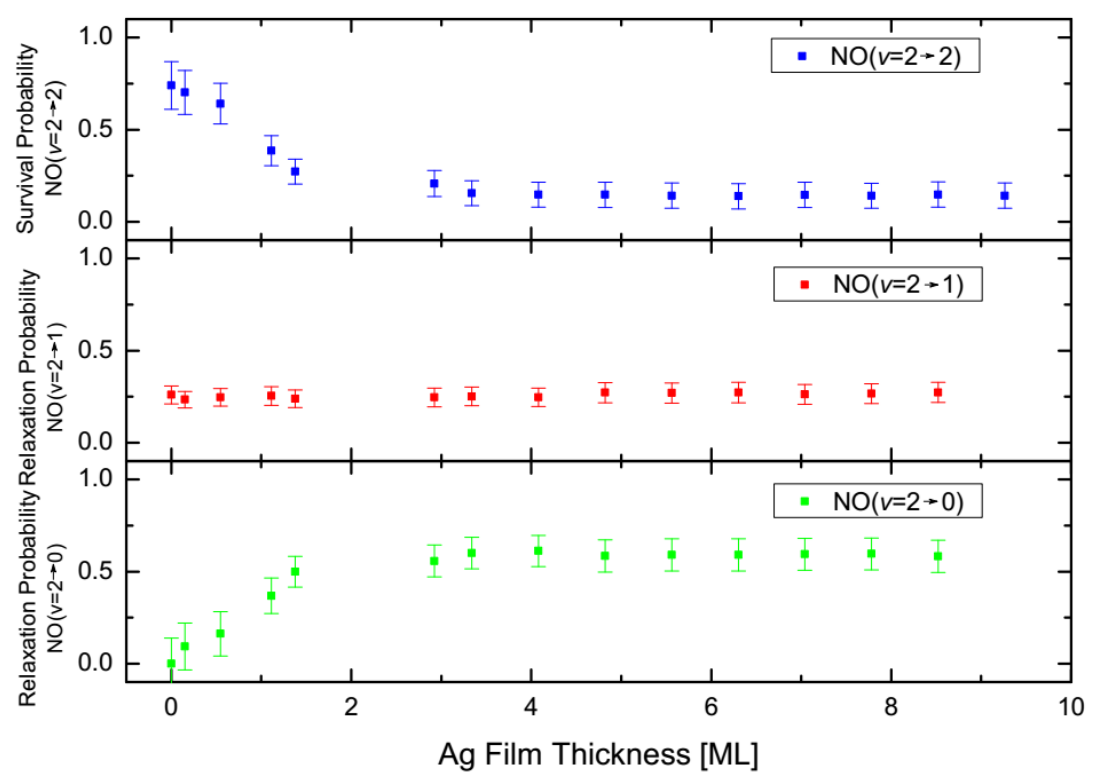

Figure 5.13: Thickness-dependent $N O(v=2)$ relaxation probabilities from Ag/Au with absolute errors due to error propagation. Note that relaxation probability $\mathrm{NO}(v=2 \rightarrow 0)$ was obtained indirectly from the experimentally accessible channels, analogously its errors bars were calculated by error propagation from the errors of $\mathrm{NO}(v=2 \rightarrow 2)$ and $\mathrm{NO}(v=2 \rightarrow 1)$.

A rigorous error propagation to calculate the error of the absolute relaxation probabilities was carried out taking into account all errors included in equation (5.6) (the error of the absolute relaxation probability on $\mathrm{Au}(111)$, error of the signal measurements and the errors of the three correction factors), the results are shown in Figure 5.13. The probability of the $\mathrm{NO}(v=2 \rightarrow 2)$ channel shows error bars between $7-13 \%$, the $\mathrm{NO}(v=2 \rightarrow 1)$ channel errors of $5-6 \%$.

Note that systematic errors are not considered. During the determination of absolute scattering probabilities of $\mathrm{NO}(v=2)$ scattering from $\mathrm{Au}(111)$, relaxation into $v=0$ was neglected which might introduce a small error. It has been observed that NO in higher incidence states exhibits a stronger relaxation, therefore the $\mathrm{NO}(v=3)$ data might be invoked to estimate an upper limit of the error introduced by this assumption. Branching ratios of $\mathrm{NO}(v=3)$ have been measured on the same machine [92]. For a similar incidence energy $(0.65 \mathrm{eV})$ that state exhibited a survival probability of $43 \%$, relaxation into $v=2$ was reported with $39 \%$ and the remaining $18 \%$ into $v=1$. Let us imagine that the $\mathrm{NO}(v=3 \rightarrow 1)$ channel would not be accessible. How big would the error be? If we assumed for that measurement that this lowest state $(v=1)$ were not experimentally accessible, an error of $<20 \%$ would be introduced. If $\mathrm{NO}(v=2)$ would show a similar tendency for relaxation, that would be probably an upper limit for this error. However, as already mentioned, the tendency to relax is strongly dependent on the incident vibrational state and $\mathrm{NO}(v=2)$ shows a significantly lower relaxation probability. Estimation is possible by comparing the ratio between molecules in the initial state and those which lose one vibrational quantum: For $\mathrm{NO}(v=3)$, the ratio of $\mathrm{NO}(v=3 \rightarrow 2) / \mathrm{NO}(v=3 \rightarrow 2)$ is about $1: 1$ whereas for $\mathrm{NO}(v=2 \rightarrow 2) / \mathrm{NO}(v=2 \rightarrow 1)$ a ratio of $3: 1$ is calculated. A maximum error of $5 \%$ is probably a good estimation for an upper limit when neglecting the $\mathrm{NO}(v=2 \rightarrow 0)$ scattering channel on $\mathrm{Au}(111)$. This would not change the general trend, but rather lead to slight systematic shifts: Probabilities of the $\mathrm{NO}(v=2 \rightarrow 2,1)$ channels would be reduced and the $\mathrm{NO}(v=2 \rightarrow 0)$ channel increased by a few percent. 


\subsection{Discussion}

The main characteristic of the film thickness dependent relaxation probability of $\mathrm{NO}(v=2)$ is its strong increase of from $16 \%$ to $86 \%$ which is found between 0 to $3 \mathrm{ML} \mathrm{Ag} /$ Au until achieving a constant value for higher thicknesses. Moreover, in combination with the thickness-independent trend of the relaxation probability via the $\mathrm{NO}(v=2 \rightarrow 1)$ channel, it can be deduced that the for thick silver films about $60 \%$ lose two vibrational quanta and relax into the ground state. In the following section, the results will be compared to previous measurements followed by a discussion about mechanisms that could describe the observed behavior.

\subsubsection{Direct Scattering vs. Trapping Desorption}

When molecules are scattering from the surface, two different pathways of collision can be envisioned: The first case is when the impinging molecules undergo a translationally inelastic single collision and are immediately reflected. Molecules following that mechanism termed as "direct (inelastic) scattering" (DS) are found in a non-thermal rotational distribution. An alternative mechanism is "trapping desorption" (TD) for molecules which trap, completely equilibrate at the surface and leave the surface after desorption. Both mechanism were described in one of the first UHV gas-surface scattering experiments using a Xe atom beam and a Pt(111) surface [3]. The high amount of molecules that relax into the ground vibrational state rises the question if trapping desorption might explain the observed behavior. However, this mechanism can be excluded which is supported by the following facts: The incidence energy of $0.59 \mathrm{eV}$ was chosen that the trapping was negligible according to a study of NO scattering from $\mathrm{Ag}(111)$ [93]. All angular distributions are narrow and peak close to the specular angle (see Figure 5.6), as expected for a direct scattering mechanism. The mean translational energies from $\mathrm{NO}(v=0 \rightarrow 0)$ scattering experiments are strongly exceeding the thermal limit $\left(k_{\mathrm{B}} T=0.026 \mathrm{eV} @\right.$ room temperature) which would be expected for a TD mechanism (see Figure 4.4). As final test, a faster gas mixture with a higher translational incidence energy $\left(\mathrm{E}_{\mathrm{i}}=0.95 \mathrm{eV}\right)$ was prepared. This mixture is too fast to be trapped in a physisorption well. When the same REMPI signal measurement was carried out for different film thicknesses, the results (Figure 5.14) showed no difference to the trend observed with the usual mixture $\left(E_{i}=0.59 \mathrm{eV}\right)$. If TD on a Ag-coated surface were the reason for the REMPI signal decrease for the $0.59 \mathrm{eV}$ mixture, a smaller decrease would be expected on a Ag surface using the mixture with $0.95 \mathrm{eV}$. As a conclusion, a trapping desorption mechanism can be ruled out in our experimental conditions, therefore the observed NO scattering in this work will be attributed to direct scattering. 


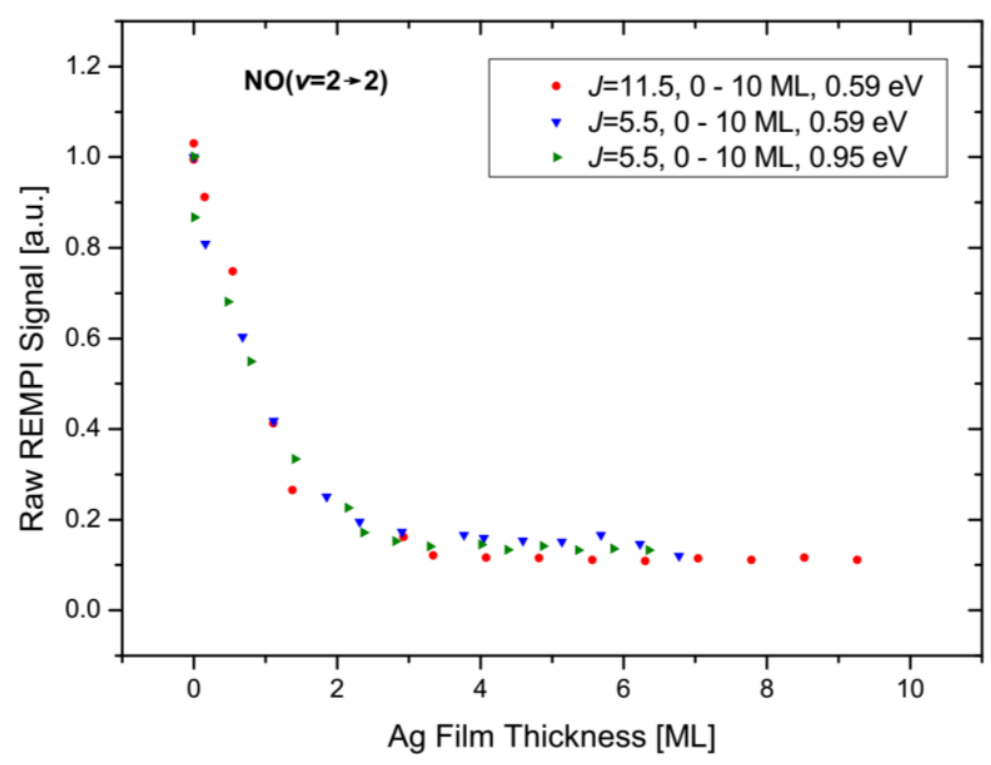

Figure 5.14: No sign of trapping desorption: $N O(v=2 \rightarrow 2)$ signal intensity from mixture with 0.95 eV incidence energy shows the same behavior as the standard mixture with $E_{i}=0.59 \mathrm{eV}$.

\subsubsection{Role of Surface Characteristics for Vibrational Energy Transfer on Metal Surfaces}

The vibrational energy transfer of NO scattered from metal surfaces is a well-established example for electronically nonadiabatic interactions between molecular vibration and the electronic system of the surface. The important role of surface electrons in vibrational relaxation processes was found when comparing the state distributions of vibrationally excited NO scattered from LiF and $\mathrm{Au}(111)$ [43]. Scattering from the insulator surface LiF showed almost no vibrational relaxation, however strong multiquantum relaxation leading to a broad vibrational state distribution was found when scattering NO from the metallic $\mathrm{Au}(111)$ surface. Multiquantum relaxation from metal surfaces is understood as an electron-mediated process which includes the formation of a transient $\mathrm{NO}^{-}$anion $[43,94]$. As the NO molecule approaches the surface, its affinity level is energetically lowered due to image charge stabilization. When crossing the Fermi level, it can accept an electron, allowing an efficient mechanism for vibrational relaxation.

The motivation of this work, the determination of relaxation probabilities of an excited molecule after scattering from $\mathrm{Au}(111)$ and ultrathin metallic films, is to quantify the nonadiabatic interaction between a molecule and a defined surface. Furthermore, by comparing the scattering behavior from different defined surfaces, it might be possible to identify surface properties which are important to the nonadiabatic interaction processes. In this context, the observations of this work, the increase of the relaxation probability of $\mathrm{NO}(v=2)$ scattered off silver film within the growth of the first monolayers, can be interpreted as increasing nonadiabatic coupling between molecule and surface. With the assumption that films above $3 \mathrm{ML} \mathrm{Ag/Au} \mathrm{behave} \mathrm{like} \mathrm{scattering} \mathrm{from} \mathrm{bulk} \mathrm{Ag}(111)$, this work can be juxtaposed to a previous relaxation study on both materials. In this investigation, the vibrational state distribution after scattering of $\mathrm{NO}(v=11)$ from both surfaces was acquired [48]. 


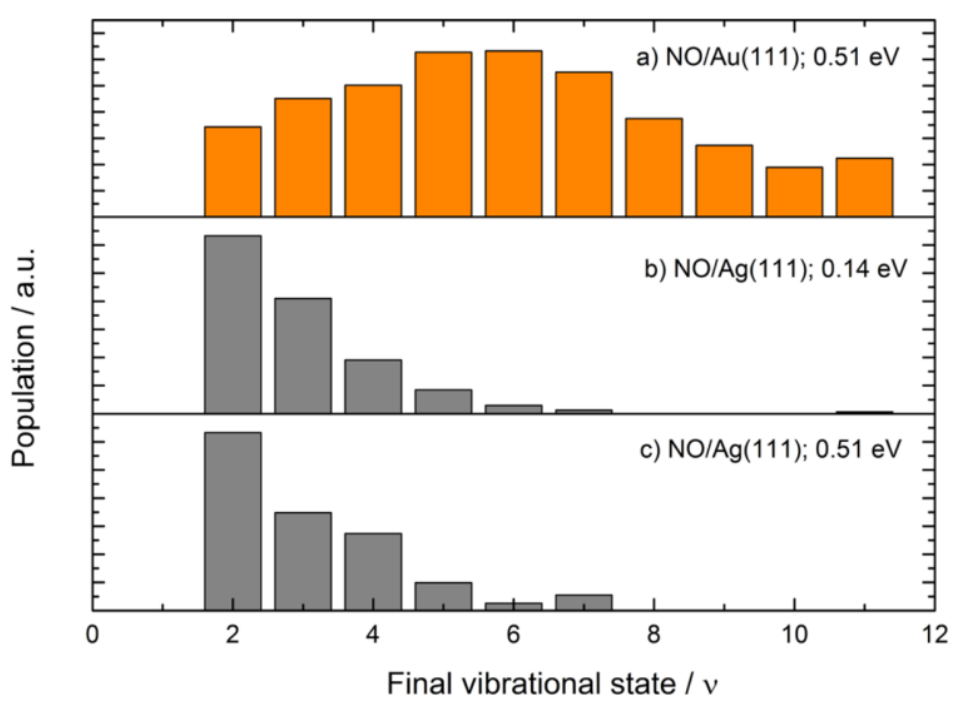

Figure 5.15: Relaxation of highly excited $N O(v=11)$ from $A u(111)$ and $A g(111)$, taken from reference [48].

The results of those measurements are depicted in Figure 5.15. Ignoring the small difference in incidence energy, on a qualitative level scattering of $\mathrm{NO}(v=2)$ and $\mathrm{NO}(v=11)$ show a consistent behavior with regard to the surfaces. The relaxation is substantially increased on $\mathrm{Ag}(111)$ in comparison to $\mathrm{Au}(111)$. Both distributions of $\mathrm{NO}(v=2)$ and $\mathrm{NO}(v=11)$ scattered off a silver surface peak in the lowest vibrational state which was observed in the experiment.

Two explanations for the higher relaxation probability on $\mathrm{Ag}(111)$ were proposed in the previous study. The favored hypothesis was the difference of the NO dissociation barriers on both materials. Due to the high amount of vibrational energy of $\mathrm{NO}\left(v=11, \mathrm{E}_{\text {vib }}=2.5 \mathrm{eV}\right)$, the total energy $(T+V=3.0 \mathrm{eV})$ is rather close to the energy of the dissociation transition state of $\mathrm{NO}$ on $\mathrm{Ag}(111)$ (3.1 eV) in comparison to $\mathrm{Au}(111)(3.6 \mathrm{eV})$. NO trajectories with a stretched geometry are more likely to occur which exhibit higher susceptibility to electrons, thereby facilitating nonadiabatic coupling and vibrational relaxation [47]. The same argument was used to explain the increase of an analogous vibrational transfer process, the vibrational excitation of $\mathrm{HCl}(v=1 \rightarrow 2)$ in comparison to $\mathrm{HCl}(v=0 \rightarrow 1)$ [41] due to a to smaller energetic distance to the dissociation barrier. However, in the present work, that condition is not fulfilled. The investigated vibrational state $\mathrm{NO}\left(v=2, \mathrm{E}_{\mathrm{vib}}=0.46 \mathrm{eV}\right)$ has a significantly lower vibrational energy. If a near-dissociation transition state would play a role in vibrational transfer of $\mathrm{NO}(v=2)$, it would be expected to observe a strong difference when comparing vibrational excitation of $\mathrm{NO}(v=0 \rightarrow 1)$ and $\mathrm{NO}(v=2 \rightarrow 3)$ similar to the effect observed for $\mathrm{HCl}$. However, the excitation of $\mathrm{NO}(v=2 \rightarrow 3)$ only shows a small enhancement over $\mathrm{NO}(v=0 \rightarrow 1)$. Therefore, the influence of the dissociation barrier does not seem to be a valid explanation for the increased relaxation of $\mathrm{NO}(v=2)$ on $\mathrm{Ag} / \mathrm{Au}$.

An alternative explanation for the increased relaxation on $\mathrm{Ag}(111)$ is the difference of the work functions between $\mathrm{Au}(111)$ (5.26 eV [38] - $5.55 \mathrm{eV}$ [11]) and Ag(111) (4.46 eV [37] - $4.72 \mathrm{eV}$ [95]). Early considerations when comparing the probability of vibrational excitation of $\mathrm{NO}(v=0 \rightarrow 1)$ on a $\mathrm{Au}, \mathrm{Ag}$ and Cu surface lead to the conclusion that the work function might be used as an intuitive proxy helping to quantify nonadiabatic interactions [18]. The difference between molecular affinity level and work function defines a barrier which the electron has to overcome. Accordingly, a lower work function would facilitate an efficient relaxation process. Calculations with the IESH model [96], a theoretical approach to describe molecular-surface scattering by including nonadiabatic effects, provided additional support for this idea: The model predicted that $\mathrm{NO}(v=11)$ relaxation from $\mathrm{Au}(111)$ was increased when the work function was lowered to $4.7 \mathrm{eV}$. 
The thickness-dependent relaxation behavior of $\mathrm{NO}(v=2)$ measured in this work strongly supports the hypothesis that the increased relaxation is dominated by the change of the surface work function. Several groups reported the work function change of the $\mathrm{Ag} / \mathrm{Au}(111)$ system at different film thicknesses $[11,12]$ showing a strong decrease within the first monolayers before reaching the value measured for the bulk case, a similar trend as observed for the survival probability. A linear trend is observed for thicknesses between completed monolayers.

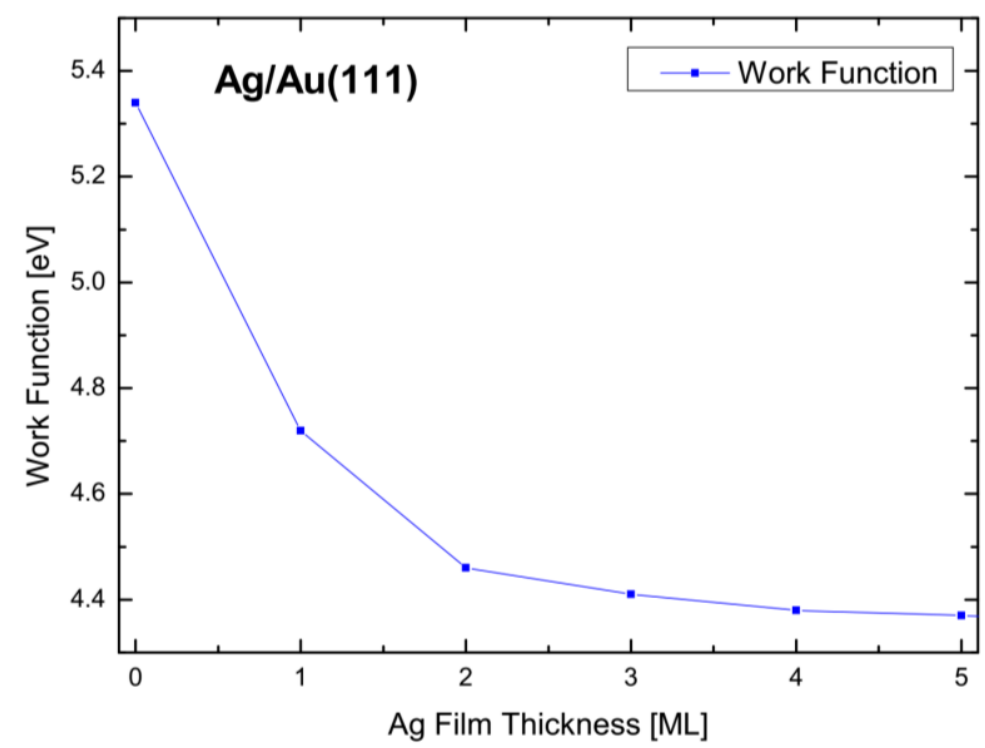

Figure 5.16: Work function at different silver film thicknesses of Ag/Au(111), data taken from Ref. [12].

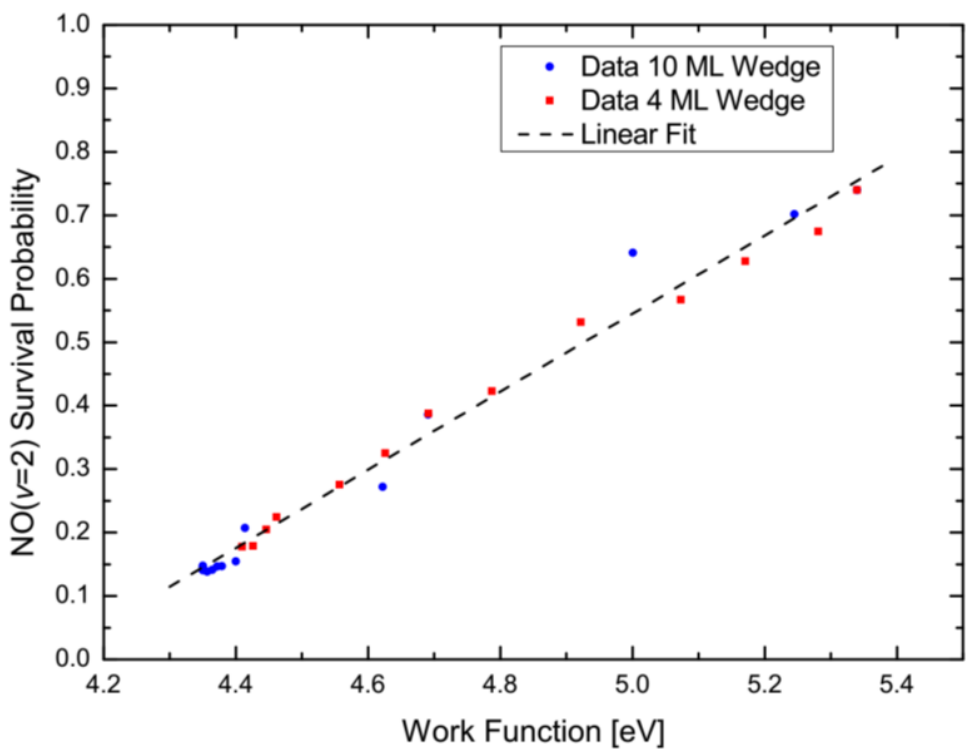

Figure 5.17: Survival probability for different work function (conversion according to the thickness-dependent work function of Ref. [12]). A linear correlation between both parameters is observed.

For the presented data, the most recent dataset by Cercellier et al. (see Figure 5.16) was used to determine work function from the film thicknesses data and the survival probability was plotted against this parameter. The result is shown in Figure 5.17 and suggests a linear correlation between the work function and the survival probability of $\mathrm{NO}(v=2)$. Quantitatively, in this interpretation, a work function change of $0.1 \mathrm{eV}$ would correspond to a change of $\sim 6 \%$ survival probability. 
Regarding the fine structure of the thickness-dependent work function, an interesting feature is found concentrating on the characteristics close to the completion of a monolayer: From Figure 5.16 we see that the $\mathrm{Ag} / \mathrm{Au}$ work function during the formation of a layer is following a linear trend. As a consequence, at a thickness of completed coverage (e.g. 1, $2 \mathrm{ML} \mathrm{Ag/Au}$ ) a kink can be spotted, similar as in the Auger signals (compare to Figure 3.8 and Figure 3.9). Measurements of the $\mathrm{NO}(v=2 \rightarrow 2)$ REMPI signal revealed a similar kink characteristic. To obtain the signal intensity with high point density at several stages during the growth a monolayer, low-gradient wedges of 0-2 ML $\mathrm{Ag} / \mathrm{Au}(111)$ and 1$3 \mathrm{ML} \mathrm{Ag} / \mathrm{Au}(111)$ were prepared. For a wedge of 0-2 ML thickness, the data (Figure 5.18, left panel) could be fitted very well by two straight lines exhibiting a kink at about $1 \mathrm{ML}$ film thickness. An alternative fitting procedure employing an exponential decay function resulted in an increased root mean square error by a factor of 2 . The same applies to signal data from a wedge of 1-3 ML (Figure 5.18 , right panel): fitting with two single lines gives better agreement than exponential decay.
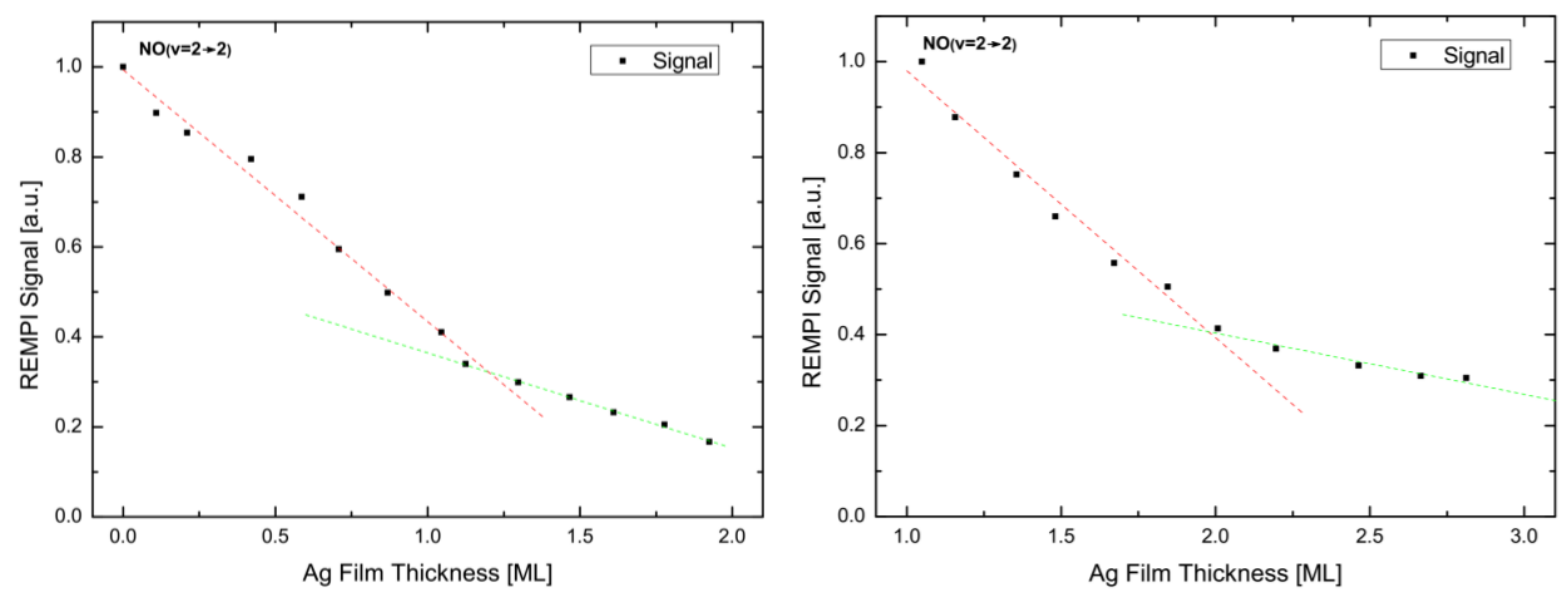

Figure 5.18: REMPI signal measurements from low gradient wedges showing a kink after monolayer formation. Left panel: $\mathrm{NO}(v=2 \rightarrow 2)$ signal intensity along a low gradient wedge of 0-2.4 ML Ag/Au. The data can be fitted very well by two subsequent straight lines. Right panel: $N O(v=2 \rightarrow 2)$ signal intensity along a wedge of $1-3 \mathrm{ML} \mathrm{Ag/Au}$. Please note that different to the usual procedure, the signal at $\sim 1 M L A g / A u$ is used as reference.

Admittedly, since the REMPI signal does get affected by changes of the angular distributions, the width of the arrival time distribution and the final molecular velocity, one might assign the observed behavior to those factors. If the slope of one correction factor $\frac{d C F}{d T}$ is changing between the first and the second monolayer for one of those three aspects, that could explain the kink observed in the REMPI signal as well. However, regarding the correction due to broadening of the TOF distribution and the density to flux conversion, this is not observed. Within experimental resolution, the available data suggest rather a linear trend for the range of 0-2 ML unaffected of the completion of the monolayer. In regard to the remaining aspect, angular distribution and the corresponding correction factor has not been acquired with such a point density that a kink behavior can be ruled out. On the contrary, with the way of interpolating between the obtained points, it seems that a kink can be spotted for that trend. However, when applying the angular distribution correction as outlined in the corresponding section, the observed kink does not flatten out with the consequence that only the change of the angular distribution seems insufficient in explaining the kink observed in the signal data.

To conclude, the silver surfaces between 0-3 ML Ag/Au exhibit a strong increase in relaxation, a thickness region where the surface work function is known to show significant change. Converting the parameter film thickness into work function based on experimental data, the survival probability plotted against work function suggests a linear correlation. Finally, the observation of kinks at completion of a monolayer, a feature often found in the context of electronic properties, strongly 
supports the explanation that the strong relaxation of $\mathrm{NO}(v=2)$ on silver films is dominated by the change of the surface work function.

\subsubsection{Mechanistic Discussion: Influence of the Work Function on Vibrational Relaxation}

For the relaxation of vibrationally excited $\mathrm{NO}(v=16)$ on $\mathrm{Au}(111)$ [43], a "vibrational auto-detachment" mechanism was suggested, following ideas by Newns successfully employed to describe the excitation of $\mathrm{NO}$ from $\mathrm{Ag}(111)$ [31]. It is presumed that $\mathrm{NO}(v=2)$ will follow the same principles even if the relaxation will be not as efficient as in the previous case.

Before NO can undergo relaxation, it has to accept an electron via its $\pi^{*}$ orbital which is only possible in an energetically favored situation. When close to the surface, an electron may hop from the surface to the vibrating molecule at its outer classical turning point, forming a transient anion stabilized by its image charge. After compression to the inner turning point the energetic situation is destabilized leading to subsequent loss of the electron back to the solid.

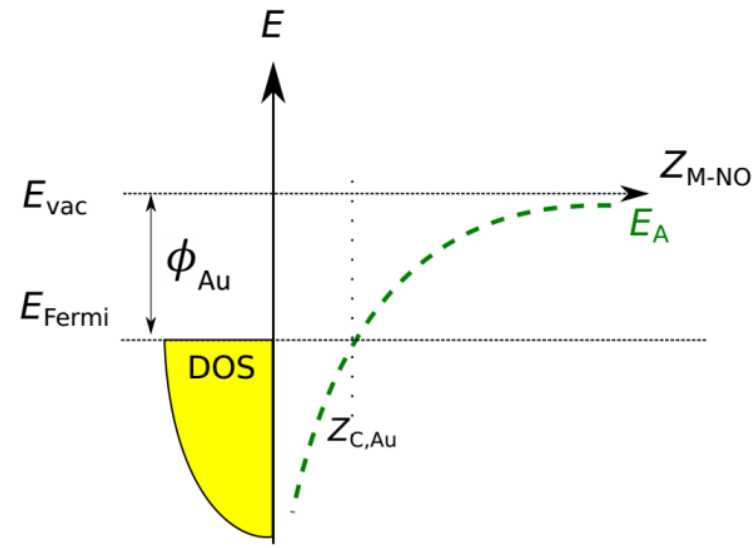

$\mathrm{Au}(111)$

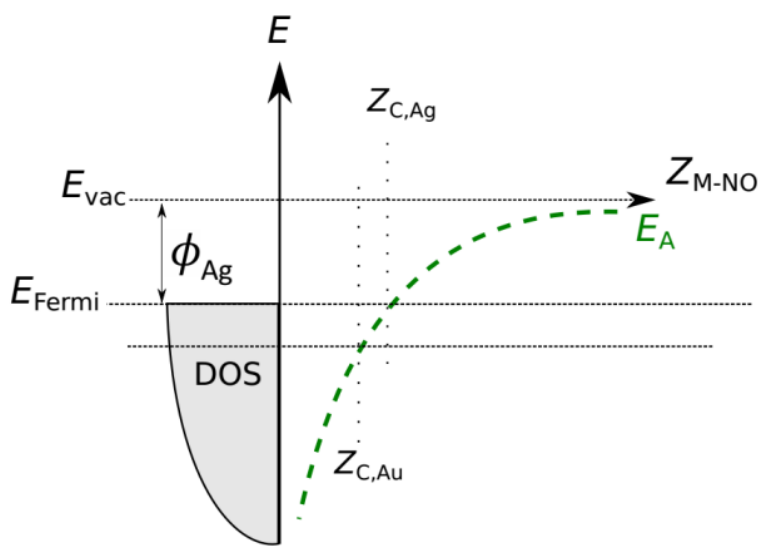

$\mathrm{Ag} / \mathrm{Au}(111)$

Figure 5.19: Electron affinity level of NO at different molecule-surface distances (qualitative description). Left panel: At Au(111), the affinity level (green dashed line) crosses the Fermi level at a critical distance $z_{c, A u}$. Right panel: At Ag/Au(111) $\left(\phi_{A g}<\phi_{A u}\right)$, the critical distance $z_{C, A g}$ for Fermi level crossing is at farther distance to the surface.

With the aid of an energy diagram depicting the nonadiabatic relaxation process, qualitatively the effect of a lower work function can be clarified. For the sake of simplicity, a molecule with frozen bond length will be assumed. In Figure 5.19 (left panel), the electron affinity level $E_{\mathrm{A}}$ (dashed green line) of a NO molecule is plotted as a function of the molecular distance $z$ to the metal $M$ (here: Gold). Two positions are marked at the $y$-axis, one is the vacuum level $E_{\mathrm{vac}}$ set to the origin of this coordinate system, a second mark labels the Fermi level $E_{\text {Fermi }}$. By definition, the work function $\phi$ describes the energetic distance $E_{\mathrm{vac}}-E_{\text {Fermi }}$ for a defined surface.

At infinite distance, the affinity level $E_{\mathrm{A}}$ assumes the value for the isolated NO molecule in the gas phase which was experimentally determined as $0.026 \mathrm{eV}$ [97]. Note that this amount of energy is released when an electron is accepted, accordingly the vacuum affinity level is slightly stabilized towards $E_{\mathrm{vac}}$. Due to the image charge effect, the $E_{\mathrm{A}}$ level is stabilized when approaching the surface. 
However, only as starting to cross the Fermi level $E_{\text {Fermi }}$ at a critical distance $z_{\mathrm{C}}$, interaction with the metal density of states (DOS) and subsequent relaxation is energetically possible.

How different is the situation if the surface is coated with silver having a lower work function? The situation is depicted in Figure 5.19 (right panel). First, it has to be stressed that the DOS is not significantly changing, because both $\mathrm{Au}(111)$ and $\mathrm{Ag} / \mathrm{Au}(111)$ provide electronic levels of a nearly-free $s p$ band close to the Fermi level [70]. A material with a substantially different DOS could influence nonadiabatic vibrational transfer as was shown when comparing the vibrational excitation probability of $\mathrm{N}_{2}(v=0 \rightarrow 1)$ on $\mathrm{Au}(111)$ and $\mathrm{Pt}(111)$ [39]. With a different work function, the critical distance $z_{\mathrm{c}}$ is found at bigger distance to the surface. The region of nonadiabatic interaction between metal at $z=0$ and $z_{c}$ is therefore extended. A higher number of oscillations in a region with possible electron-hole pair interaction can proceed.

In a less simplified treatment that includes the molecular vibration, the previous picture needs to be extended. During oscillation of the intramolecular bond distance, the energetic distance between neutral and anionic potential is changing substantially. To accept an electron the most favored position is the outer turning point of the vibration. This geometry, as the anionic potential of NO exhibits the strongest stabilization towards the neutral, defines therefore the gateway for relaxation. In the following part of the discussion, the effect of the work function on this crucial energetic situation at the outer turning point will be visualized on the base of a ground state potential energy curve for the NO/Au(111) system (see Appendix E for details) [98].

First, the potentials will be shown with the parameters as kept in the original model, simulating NO at $\mathrm{Au}(111)$ with a work function of $5.3 \mathrm{eV}$. The two diabatic potentials of $\mathrm{NO}$ and $\mathrm{NO}^{-}$as functions of intramolecular distances $r_{\mathrm{NO}}$ and metal-NO distance $\mathrm{z}_{\mathrm{M}-\mathrm{NO}}$, are coupled in a position-dependent manner resulting in a two adiabatic potentials. A contour plot of the lower adiabatic potential is depicted in Figure 5.20 (left panel). We will put special emphasis on the energetic situation of $\mathrm{NO}(v=2)$ : In general, the black lines of the contour plot mark the parameters at different the vibrational levels $v=0-16$. The special case $\mathrm{NO}(v=2)$ is highlighted in red, showing that at sufficient distance (e.g. $z_{\mathrm{M}-\mathrm{NO}}=6 \AA$ ), the intramolecular NO distance $r_{\mathrm{NO}}$ is oscillating between 1.06 and $1.28 \AA . \AA$. In this view, translational and rotational energies are neglected.
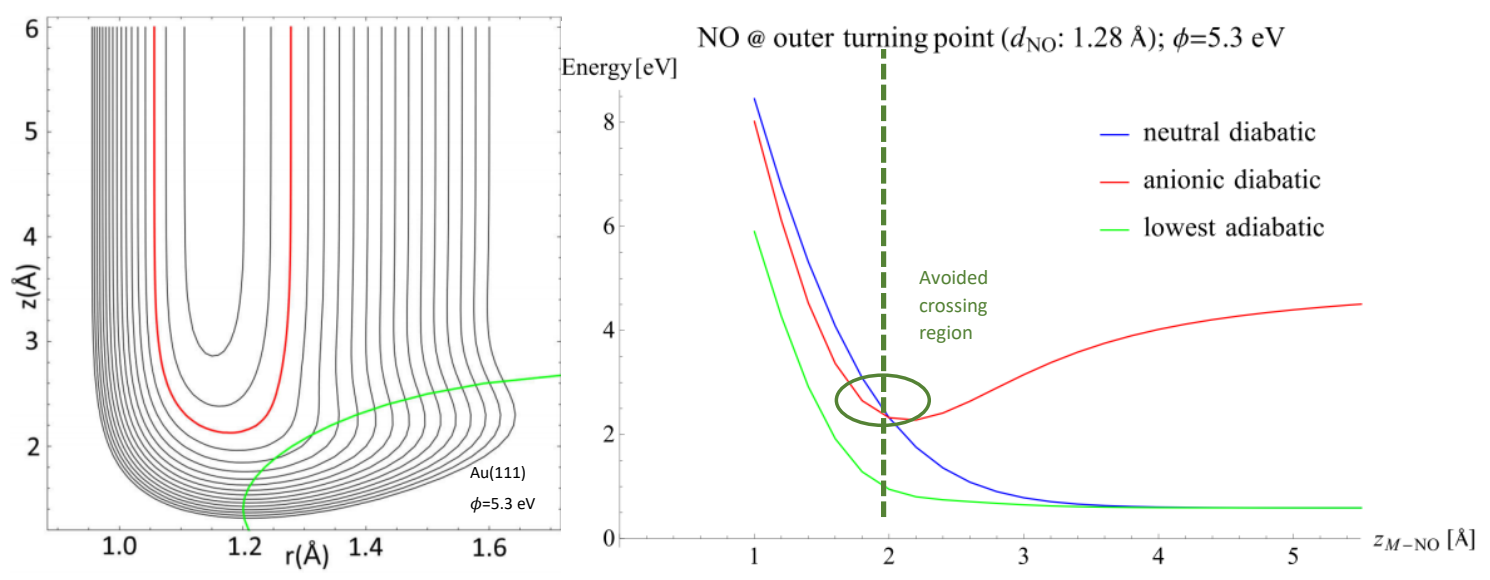

Figure 5.20: Left panel: Contour plot of the lower adiabatic potential of NO on Au(111). The energetically accessible region of $\mathrm{NO}(v=2)$ (only vibrational energy) is marked by a red line. The green line marks the parameters for which $50 \%$ ionic character of the adiabatic ground state is found. The red and the green line do not overlap. Right panel: Slice of the contour plot at fixed distance at the outer turning point of the vibration. Beside ground adiabatic potential (green), the neutral (red) and anionic (blue) adiabatic potentials are depicted. The avoided crossing region of the diabatic potentials is marked in dark green. 
The green line displays the avoided crossing region of the two diabatic curves, visualizing for which parameters $r$ and $z$ the adiabatic potential displays anionic character to $50 \%$. Within this energetic picture, a high relaxation probability is achieved when the red line and the green line overlap. However, both lines are at a certain distance, explaining the relatively weak relaxation on $\mathrm{Au}(111)$.

In the right panel of Figure 5.20, the energetic picture is shown at the fixed intramolecular distance of the outer turning point. It provides an extended description of the energetic situation in comparison to Figure 5.19. However, instead of a critical distance $z_{c}$, the distance of the avoided crossing of the two diabatic potentials is depicted. Nevertheless, both values should provide a qualitative measure at which distance to the surface the formation of the transient ion is possible with the result of strong vibrational relaxation.

For the case of a $\mathrm{Ag} / \mathrm{Au}(111)$ surface, it is assumed that the same model can be employed by modifying the parameter work function $(\phi=4.7 \mathrm{eV}$ as reported for $\mathrm{Ag}(111))$. The resulting potentials are shown in Figure 5.21.
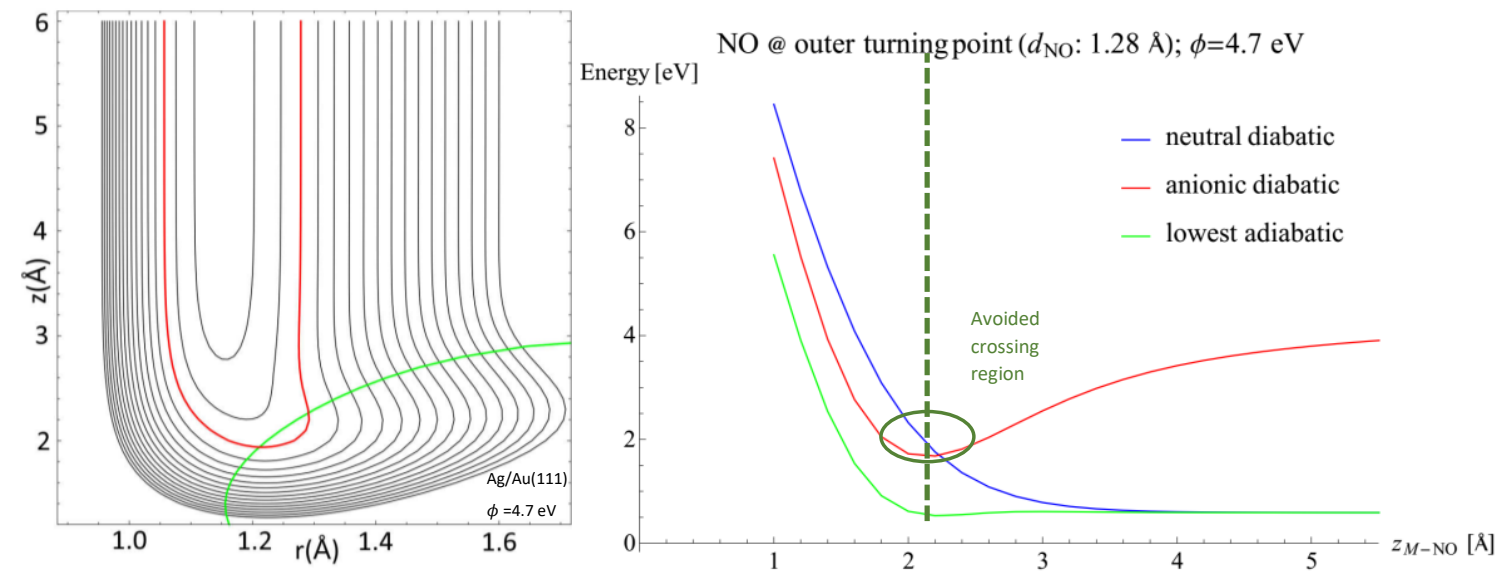

Figure 5.21: Left panel: Similar plot as in Figure 5.20, only the work function was lowered to $4.7 \mathrm{eV}$. The energetically accessible region of $\mathrm{NO}(v=2)$ overlaps with the green line demarcating the crossing of the two diabatic potentials resulting in stronger vibrational relaxation. Right panel: Slice of the contour plot at fixed distance at the outer turning point of the vibration. The anionic diabatic state is energetically lowered in comparison to Figure 5.19 (right)Figure 5.19: Electron affinity level of NO at different molecule-surface distances (qualitative description). Left panel: At Au(111), the affinity level (green dashed line) crosses the Fermi level at a critical distance $z_{C, A u}$. Right panel: At $A g / A u(111)\left(\phi_{A g}<\phi_{A u}\right)$, the critical distance $z_{C, A g}$ for Fermi level crossing is at farther distance to the surface., the avoided crossing region is shifted to a farther distance from the surface.

It is clear, that the overlap of the red and green curve is increased in comparison to the previous case (left panel). That means that the energetically available parameter space is increased for a material with a lower work function. A higher relaxation probability is therefore expected. The crossing of the red and the green lines indicates that already the pure vibrational energy of the molecule would be sufficient to observe a certain amount of relaxation. Looking at the single potential curves (Figure 5.21, right panel), the avoided crossing point is observed at farther distance from the surface, similarly as the previously invoked critical distance $z_{\mathrm{c}}$ (Figure 5.19).

In conclusion, within the established picture of nonadiabatic vibrational transfer, the increase of relaxation of vibrationally excited NO for a lower work function can be explained very well. In a simple qualitative picture with a fixed intramolecular distance, a lower work function increases the interaction region close to the surface, where vibrational relaxation can occur as a consequence of electron transfer. In a semi-quantitative point of view taking into account the experimental conditions and comparing it to existing potential energy curves, a lower work function increases the energetically accessible region for which the adiabatic ground state of NO is having anionic character predicting a higher vibrational relaxation. 


\subsubsection{Alternative Explanations for the Increased Relaxation Probability on $\mathrm{Ag} / \mathrm{Au}$}

When a surface is modified by deposition of a different material, this change affects not only a single but a number of different surface properties. Parallel to the effective mass experienced by the scattered molecules (see section 4.2.2) and the already discussed work function, there are other characteristics that may have an influence on the relaxation probability. One aspect is the electronic structure at a surface: In contrast to a three-dimensional bulk metal, due to the abrupt termination of the periodicity of the lattice, new electronic states are formed at the surface. Since those states are energetically close below the Fermi level, it could be imagined that they might be involved in electron transfer processes to approaching molecules. The variation of the surface state energy of the $\mathrm{Ag} / \mathrm{Au}(111)$ system $[10,11]$ with film thickness is a well-studied characteristic. Generally energy shift of surface state and work function occur simultaneously, therefore it is impossible to investigate both processes separately. However, concentrating on the beginning of film deposition between $0-1 \mathrm{ML}$ where relaxation increases most strongly, the change of work function $(480 \mathrm{meV})$ is significantly higher than the shift of the surface state $(170 \mathrm{meV})$. Therefore, a special role of surface states on the relaxation probability of vibrationally excited molecules seems unlikely.

Furthermore, the influence of a further electronic effect could be ruled out. One motivation of this study was to increase the density of states close the Fermi level by growing thin films structures which exhibit confined electronic states known as quantum well states found in $\mathrm{Ag} / \mathrm{Au}(111)$ film for thicknesses between 7-40 ML [10,70]. $\mathrm{NO}(v=2)$ scattering from a thin film wedge including higher film thicknesses (5-35 ML) did not show any deviations from the behavior observed for thicknesses > $5 \mathrm{ML}$ (compare 5.1.1).

Another effect which could lead to an increased NO relaxation is a significant difference between the potential energy surfaces for the interaction with $\mathrm{Au}(111)$ and $\mathrm{Ag}(111)$. One aspect of the interaction between a molecule and a surface is the desorption energy which can be determined from temperature programmed desorption (TPD) experiments. In a recent study in our group, a desorption peak of $\mathrm{NO} / \mathrm{Au}(111)$ was detected at $T_{\text {des }}=65 \mathrm{~K}$ with a desorption energy of $0.23( \pm 0.02) \mathrm{eV}$ [99]. While NO remains unreactive even after adsorption on $\mathrm{Au}(111)$, decomposition is observed after adsorbing on $\mathrm{Ag}(111)$ at low temperatures, leading to a number of peaks in a TPD measurement. In the study of Behm and Brundle, one peak found at $T_{\text {des }}=100 \mathrm{~K}$ was assigned to the single monolayer desorption; probably following the method of Redhead [100], a desorption energy of $0.26 \mathrm{eV}$ was estimated. Following the same evaluation procedure on the data obtained by So [101], a slightly lower value of $0.23 \mathrm{eV}$ is obtained. From that comparison, only minor differences are observed for the adsorption well for NO on Au and Ag. However, depending on the evaluation method, TPD methods yield in strongly different results. For example, if the Redhead analysis is applied on the NO/Au(111) data (assuming a typical prefactor of $v=10^{13} \frac{1}{s}$ ), a desorption energy of $0.17 \mathrm{eV}$ is estimated. It remains unclear, if that difference is significant enough to influence the relaxation probability. It could be even imagined that a work function change is inextricably intertwined with a potential modification: When comparing the adiabatic potentials of $\mathrm{NO}$ on $\mathrm{Au}(111)$ and $\mathrm{Ag} / \mathrm{Au}(111)$ (green line in Figure 5.20 and Figure 5.21, right panel), a small potential well is visible for the lowest adiabatic potential on $\mathrm{Ag} / \mathrm{Au}(111)$ which is not observed in the case of $\mathrm{Au}(111)$.

Further theoretical studies of potential energy surfaces of NO on both materials might be able to distinguish between different effects on relaxation probability. Until those results are available, further experimental efforts on a single material but varying work function could facilitate to minimize the 
effect of a modified potential energy surface. One example would be the investigation of a special film system $\mathrm{Ag} / \mathrm{Fe}(100)$ exhibiting WF oscillations due to quantum well states very close to the Fermi level even above the $3 \mathrm{ML}$ limit observed in this work [65]. Alternatively, the work function of a surface can be reduced by auto-epitaxy of a metal upon itself. This effect known as the Smoluchowski effect [59] could be employed to modify the work function of other surfaces as well.

\subsubsection{A General Description of Vibrational Relaxation at Surfaces}

At present time, it is not understood which characteristics are important on the strength of molecular vibrational energy transfer. Although single molecule-surface systems are characterized in regard to the efficiency of vibrational energy transfer during scattering, one is still far from having a comprehensive understanding. Early general considerations about the properties controlling the strength of nonadiabatic interactions were concentrated on the comparison of vibrational excitation of diatomic molecules from a metal as a benchmark system [18]. From the limited amount of experimental data, the parameters molecular electron affinity and the surface work function were found to qualitatively describe the observed trends.

In recent years, the number of studies about vibrational relaxation is growing. Is it possible to draw a generalized picture by comparing different relaxation studies on varying surfaces? First it has to be mentioned that there is a higher degree of complexity in the aspect of relaxation. Since this process can be observed from a number of incident vibrational states, a parameter has to be found that can comprise this level of variety, an aspect that has already been discussed by Bartels et al. [46]. Additionally, different molecules might show a deviating interaction strength with a defined surface.

It seems that a comprehensive picture can be drawn when reducing the complex situation to two parameters: the first parameter is the already mentioned work function; the second is the vertical electron binding energy (VEBE) at the outer turning point which is the energy difference between the neutral and anionic PESs for molecules fixed at a specific geometry. The VEBE was first invoked in the context of vibrational relaxation of $\mathrm{NO}(v=15)$ during collision with a $\mathrm{Au}(111)$ surface to explain this system's strong relaxation probability [43]. Figure 5.22 (left panel) depicts the involved PESs as function of $r_{\mathrm{NO}}$ (the $\mathrm{N}-\mathrm{O}$ bond length). Considering the VEBE (inset B) for different intramolecular distances, it is clear that it becomes energetically favored to hop from the neutral to the anionic potential for

distances between 1.5-1.8 $\AA$. This situation can be achieved with a vibrationally excited NO molecule at the outer turning point of its vibration as marked as $r_{>}(v=15)$ in the figure. For a lower incident vibrational state, the outer turning point is associated with a lower $r_{\mathrm{NO}}$ and accordingly lower energy release. This picture does not only explain why molecules in a higher vibrational state undergo relaxation more readily, but additionally provides a characteristic measure to distinguish molecules in different vibrational states. 

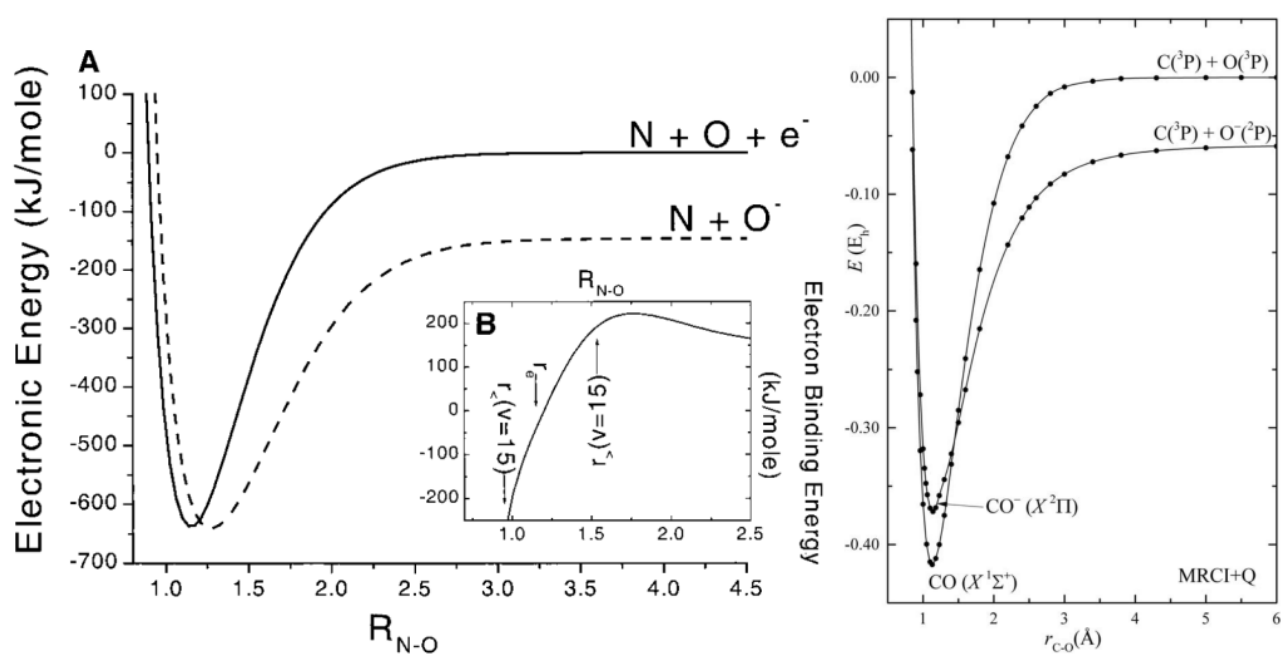

Figure 5.22: Left panel: PESS of the neutral and anionic NO (taken from Ref. [43]). Right panel: PES of neutral and anionic CO (taken from Ref. [102]).

To obtain a general picture about relaxation, several studies about relaxation of vibrationally excited NO $[43,48,92]$ and $C O[103]$ scattered from $A u$ and Ag surfaces were compared in the following manner: From the survival probability $P_{\text {surv }}$ of a system, "relaxation probability" $P_{\text {relax }}$ was calculated as (1- $\left.P_{\text {Surv }}\right)$. Moreover, using the potentials of NO [43] and CO [102] (see Figure 5.22), the VEBE at the outer turning point was calculated and subtracted by the surface work function $\phi$. For the sake of simplicity, this measure will be abbreviated as

$$
\Delta=\operatorname{VEBE}\left(r_{\text {outer }}\right)-\phi
$$

When plotting $P_{\text {relax }}$ against $\Delta$, for all datasets a common trend can be recognized (Figure 5.23).

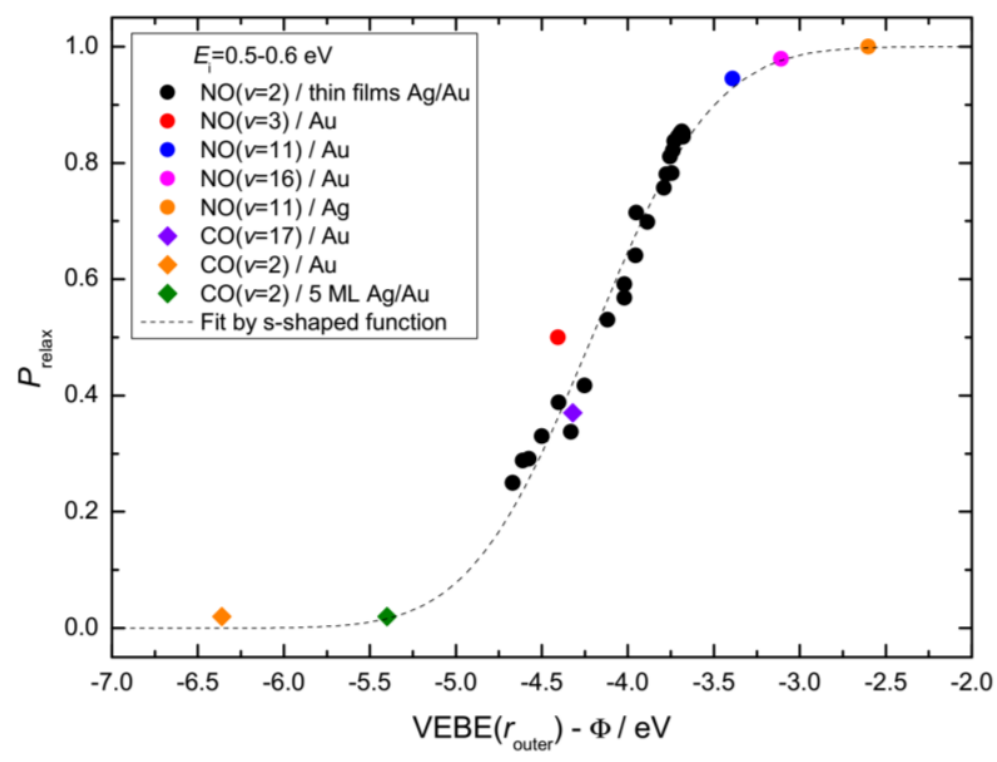

Figure 5.23: Generalized description of the vibrational relaxation (see text).

Generally, the common parameter $\Delta$ seems to be successful in predicting the strength of the relaxation probability. The extreme case of $\mathrm{CO}(v=2)$ scattering from Au and thick films of Ag shows only little tendency for relaxation and is hence set at the left of the graph. The other extreme of high relaxation probability can be found at the right section for scattering of $N O(v=11,15) / A u(111)$ and $\mathrm{NO}(v=11) / \mathrm{Ag}(111)$. The data of this work about $\mathrm{NO}(v=2)$ scattered from $\mathrm{Au}$ and thin films of $\mathrm{Ag} / \mathrm{Au}$ is found in a transition region in between. It is encouraging to find the relaxation probabilities of 
$\mathrm{CO}(v=17)$ and $\mathrm{NO}(v=3)$ in good agreement with those values. The trend resembles a s-shaped curved used typically to describe the sticking probability of a molecule on a surface for different incidence energies [104].

A theoretical explanation for this tendency might be given with the help of a potential energy surface. When changing the $\Delta$ parameter, we change the molecule's energetically accessible region on the adiabatic PES. When this region has a more anionic character, the probability to form a transient anion and hence vibrational relaxation is increased. This is exemplified in Figure 5.24 for the example of $\mathrm{NO}(v=2)$ scattering from Au and Ag/Au surfaces with three different work functions. From $5.3 \mathrm{eV}$ over $4.9 \mathrm{eV}$ to $4.7 \mathrm{eV}$, we see that the overlap between the red line, the parameter space available for $\mathrm{NO}(v=2)$, and the green line, demarcating $50 \%$ ionic character of the adiabatic ground state, overlap. The better the overlap, the higher is the relaxation probability. It is intriguing to see that for the case of a $4.9 \mathrm{eV}$ surface (Figure 5.24, center) when both lines almost begin to overlap, the $\Delta$ parameter is taking a value of -4.2 which corresponds to a relaxation probability $P_{\text {relax }}$ of 0.5 in Figure 5.23.
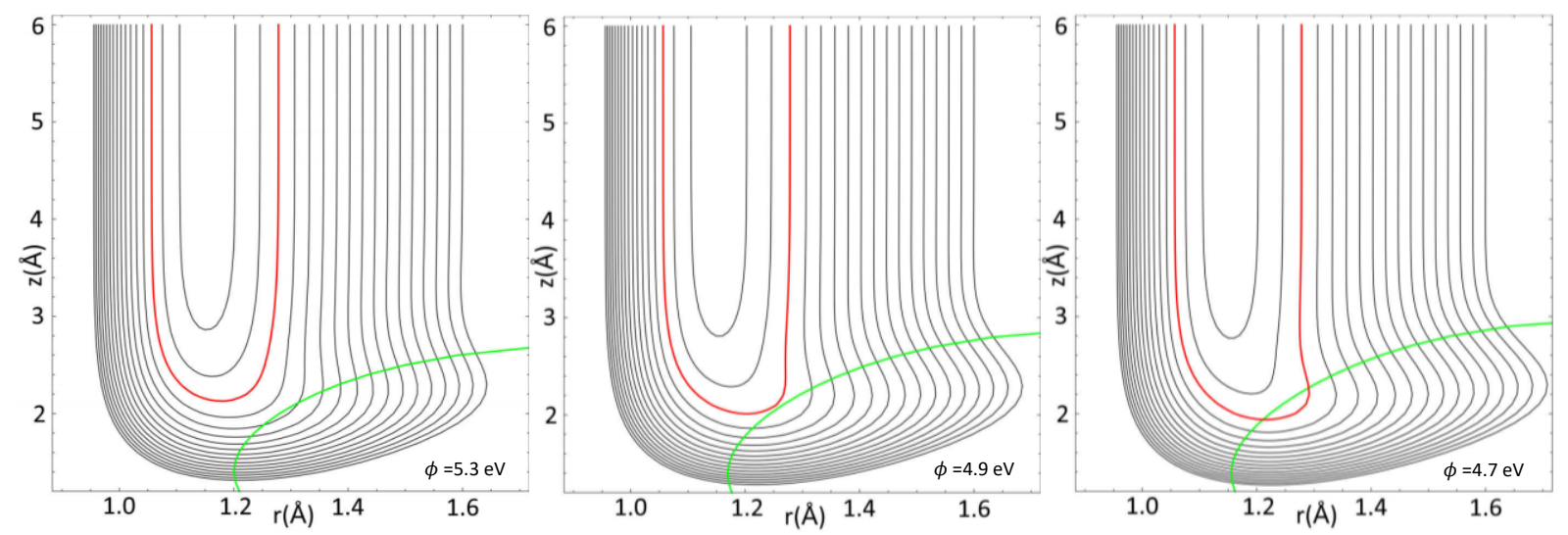

Figure 5.24: Contour plot of $\mathrm{NO}(\mathrm{v}=2)$ scattering from surfaces with different work functions (left to right: $5.3 \mathrm{eV}, 4.9 \mathrm{eV}$, $4.7 \mathrm{eV})$. From left to right, the overlap between the energetically accessible region of $N O(v=2)(r e d)$ and the region of $50 \%$ ionic character (green) increases.

The effect of higher molecular vibrational states can be recognized from the contour plots (Figure 5.24) as well. Beside the highlighted red line for $\mathrm{NO}(v=2)$, further lines are depicted for vibrational states of $\mathrm{NO}(v=0-16)$ (black). It is easy to find that a higher vibrational state increases the overlap with the green line and enhances the relaxation probability.

The data for $\mathrm{CO}(v=2)$ reveals low relaxation probabilities when scattered from $\mathrm{Au}(111)$ and thick films of $\mathrm{Ag} / \mathrm{Au}$, therefore those data points are depicted in the left of Figure 5.23. Here, the situation is modified due to a lower electron affinity $(-1.5 \mathrm{eV})$ which leads to a bigger energetic difference between both neutral and anionic diabatic potential. By adjusting the parameters of the employed model according to this molecule, it should be possible to achieve a qualitative estimation of the PES. This simulation is shown in Figure 5.25 assuming work functions of $5.3 \mathrm{eV}(\mathrm{Au}(111))$ and $4.7 \mathrm{eV}(\mathrm{Ag}(111))$. 

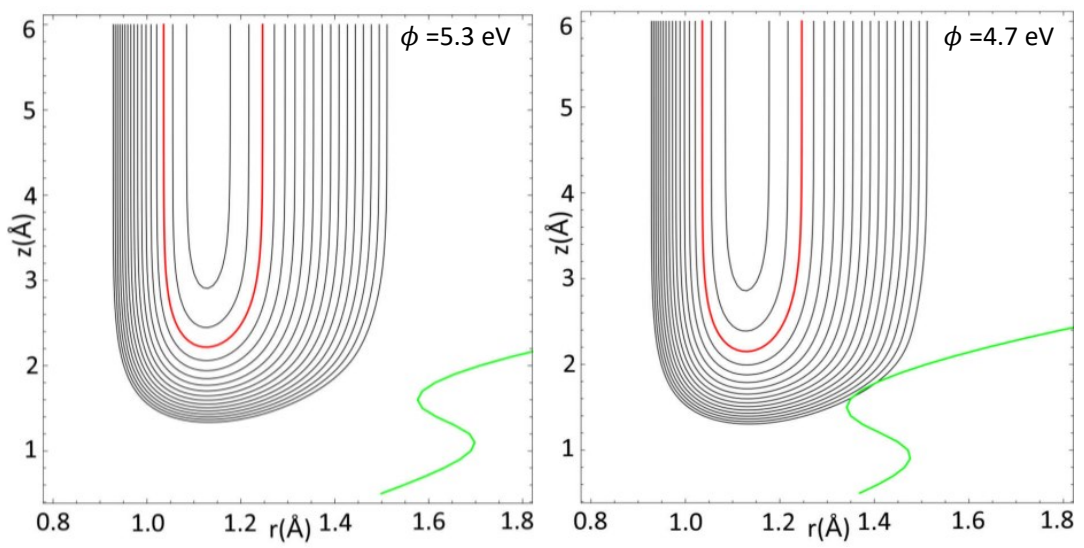

Figure 5.25: Contour plot of $\mathrm{CO}(v=2)$ scattering from surfaces assuming work functions of $\mathrm{Au}(111)$ and $\mathrm{Ag}(111)$.

It is clear for both surfaces that the red and green line are showing a significant distance, visualizing that it is less probable to achieve molecular parameters for which the adiabatic ground states exhibits strong anionic character. Thus at both surfaces the molecule $\mathrm{CO}(v=2)$ experiences only low vibrational relaxation.

Molecular vibrational relaxation is not the first nonadiabatic process that has been characterized with regard to properties of the involved molecules and surface. The nonadiabatic electron transfer step that leads to emission of anions after oxygen or halogen exposure to alkali metals is mechanistically similar [21] to the step before vibrational relaxation. The molecule $\mathrm{X}_{2}(\mathrm{X}=\mathrm{O}, \mathrm{Cl}, \mathrm{Br})$ approaches the surface and accepts an electron when crossing the Fermi level via a "harpooning mechanism", forming an $\mathrm{X}_{2}^{-}$anion. With a small probability, $\mathrm{X}_{2}^{-}$can dissociate into $\mathrm{X}^{-}$and escapes into vacuum. The probability of anion emission was experimentally determined for a number of molecule-surface systems and a trend was found when plotting this measure against the difference of the electron affinity and work function $E_{\mathrm{A}}-\phi$ which is depicted in Figure 5.26 .

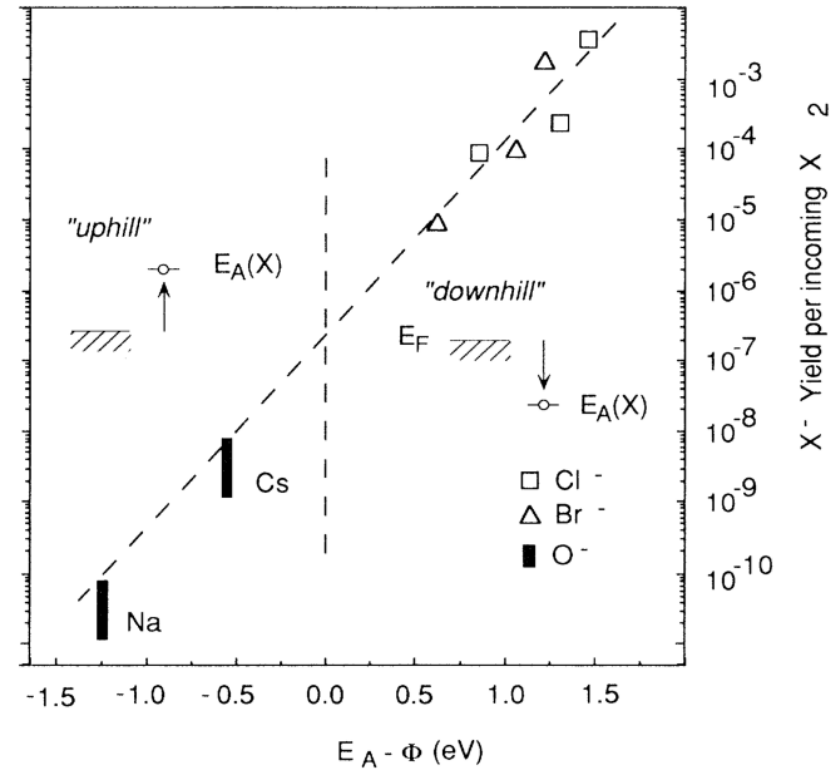

Figure 5.26: Chemical ionization yield of exoelectron emission after exposure of an alkali metal surface with $X_{2}(X=O, C l, B r)$. Plot was taken from Ref. [21]. The $x$ axis is represented similar to Figure 5.23 to show the parallel trend.

This trend of exoelectron emission yield shows a parallel behavior as observed for vibrational relaxation in Figure 5.23 which becomes clear when we compare the $x$ axes of both plots. Instead of the VEBE, the electron affinity is employed which is a more general concept. We can see the similarity 
if the write out the relation between VEBE and $E_{\mathrm{A}}$. For a diatomic molecule with bond length $r, E_{\mathrm{A}}$ is the energy difference between the minima of the neutral and anionic potential. If those potentials are implemented by two functions $V_{\text {neutral }}(r)$ and $V_{\text {anion }}(r)$ with their minima at to zero, VEBE can be defined as:

$$
\operatorname{VEBE}\left(r_{\text {outer }}\right)=E_{\mathrm{A}}+V_{\text {neutral }}\left(r_{\text {outer }}\right)-V_{\text {anion }}\left(r_{\text {outer }}\right)
$$

Accordingly, if we neglect any dependence on the bond length $r, \mathrm{VEBE}$ and $E_{\mathrm{A}}$ are describing the same characteristic of a molecule making Figure 5.23 and Figure 5.26 comparable.

In the light of our current understanding of nonadiabatic processes, the picture of Figure 5.23 gives a generalized trend of the amount of vibrational relaxation during scattering processes. This raises the question: How versatile is that description and can it be extended to other systems? One particular aspect that still has to be tested is the role of $d$-orbitals of a metal. The continuum of electronic states close to the Fermi level of $\mathrm{Ag}$ and $\mathrm{Au}$ is defined by the $s p$-band [70]. As was shown during the comparison of $\mathrm{N}_{2}$ excitation from $\mathrm{Au}(111)$ and $\mathrm{Pt}(111)$, the existence of a high density of states due to $d$-orbitales close to the Fermi level might influence the tendency to undergo nonadiabatic interactions [39]. Accordingly, vibrational relaxation could be increased on a metal like Pt(111) although its work function is above $\mathrm{Au}(111)$.

Summing up, it is possible to find a general trend for relaxation of vibrationally excited NO and CO from $\mathrm{Au}$ and $\mathrm{Ag}$ surfaces. The relaxation probabilities can be plotted against a parameter $\Delta$, the difference between vertical electron binding energy (VEBE) and the surface work function $\phi$. On the basis of calculated potential energy surfaces, the trend can be understood. For a defined $\Delta$, the molecule's energetically accessible region on the adiabatic PES is set by which regions of higher anionic character are achievable that lead to enhanced relaxation. 


\section{Vibrational Inelasticity of $\mathrm{CO}(v=2)$ after Scattering from $\mathrm{Ag} / \mathrm{Au}(111)$}

After the characterization of the $\mathrm{NO}(v=2)$ scattering from $\mathrm{Ag} / \mathrm{Au}(111)$, the scattering behavior of a further diatomic molecule was investigated: $\mathrm{CO}$.

For gaining insight about nonadiabatic vibrational transfer at surfaces, many insights were obtained from experiments with NO. Due to its simple and efficient detection scheme, this molecule was and is still used as a "work horse" for molecular beam studies. It was therefore interesting to switch to a molecule which might exhibit a different behavior. Are the trends observed for $\mathrm{NO}$ applicable to $\mathrm{CO}$ as well? Can a general idea be introduced that is able to describe the relaxation behavior for all diatomic molecules?

\subsection{Results}

The measurement techniques are very similar to the one used to characterize the relaxation of $\mathrm{NO}(v=2)$ as described in chapter 5 . After acquisition of relative REMPI signals for different film thicknesses (and $\mathrm{Au}(111)$ as common reference), a quantitative correction will be performed according to experimentally obtained data. Finally, by scaling with the relaxation probability measured for scattering from $\mathrm{Au}(111)$, absolute scattering probabilities of $\mathrm{CO}(v=2)$ from the thin film system can be determined. As described in detail in the experimental section, the observed REMPI signals $\mathrm{CO}(v=2 \rightarrow 2,1)$ were measured at the peak of the corresponding vibrational band which consists of group a rotational states. The incidence energy of the $\mathrm{CO}$ gas mixture is $0.64 \mathrm{eV}$, similar to the one of the NO mixture $(0.59 \mathrm{eV})$ used in chapter 5.

\subsubsection{Acquisition of $\mathrm{CO}(v=2 \rightarrow 2)$ and $\mathrm{CO}(v=2 \rightarrow 1)$ REMPI Signals}

The thickness-dependent REMPI signals of the $\mathrm{CO}(v=2 \rightarrow 2)$ scattering channel are displayed in Figure 6.1. At first, a signal decrease can be observed for low coverages of silver until reaching a minimum at $1 \mathrm{ML} \mathrm{Ag/Au}$. With increasing film thickness however, the trend turns around and the signal rises again. This is a strong difference in comparison with the $\mathrm{NO}(v=2 \rightarrow 2)$ signal which exhibited further signal decrease until a film thickness of $3 \mathrm{ML}$. The detection scheme for $\mathrm{CO}$ is not as efficient as for NO, leading to a lower signal to noise ratio even after with a laser power correction. As a consequence, the thickness from which a constant signal is obtained cannot be determined with the same precision. An overview over all data including measurements at higher thicknesses shows that a constant behavior can be observed from about $5 \mathrm{ML} \mathrm{Ag/Au}$. In spite of the higher statistical error, all measurements of both channels $\mathrm{CO}(v=2 \rightarrow 2,1)$ exhibit high consistency over time; no signal decrease with time was detected as previously for the $\mathrm{NO}(v=2 \rightarrow 1)$ channel. 


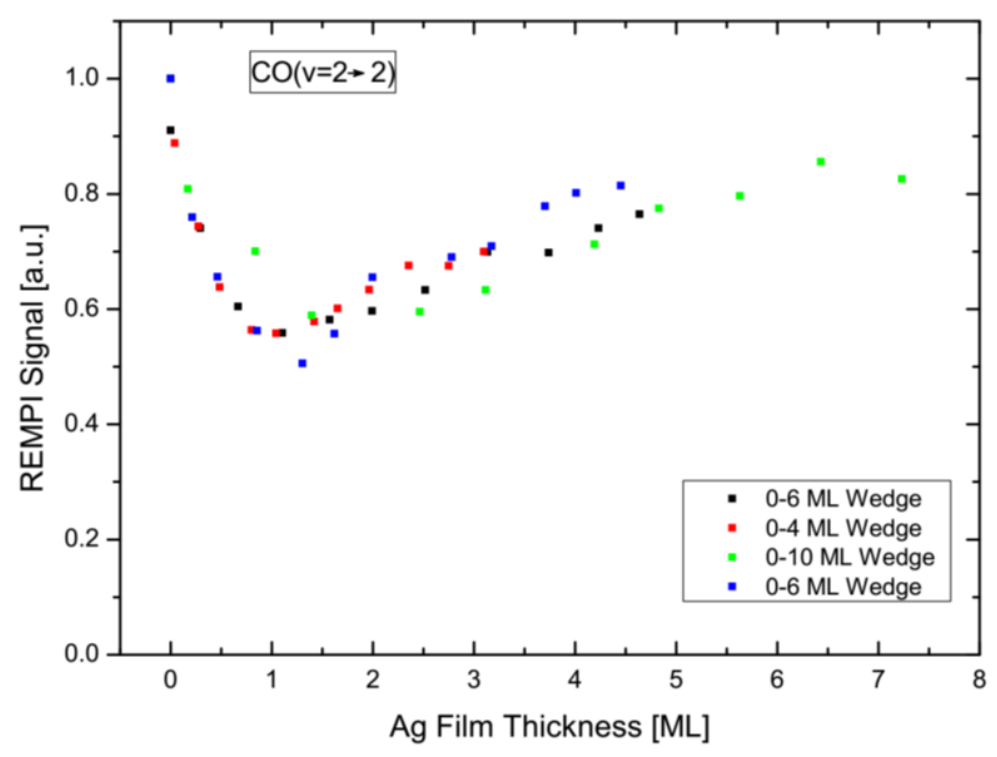

Figure 6.1: REMPI Signal of the $\mathrm{CO}(v=2 \rightarrow 2)$ scattering channel from wedges of different thickness gradients.

In an analogous manner, the REMPI signals of the vibrationally inelastic scattering channel $\mathrm{CO}(v=2 \rightarrow 1)$ were acquired (Figure 6.2). Between 0-1 ML Ag/Au, the signal remains approximately constant. From $1 \mathrm{ML}$ film thickness on, surprisingly, a signal increase by a factor of about 2.3 is observed. This indicates that $\mathrm{CO}(v=2)$ relaxation is increased on a silver surface and that a significant amount of molecules relaxes into the $v=1$ channel.

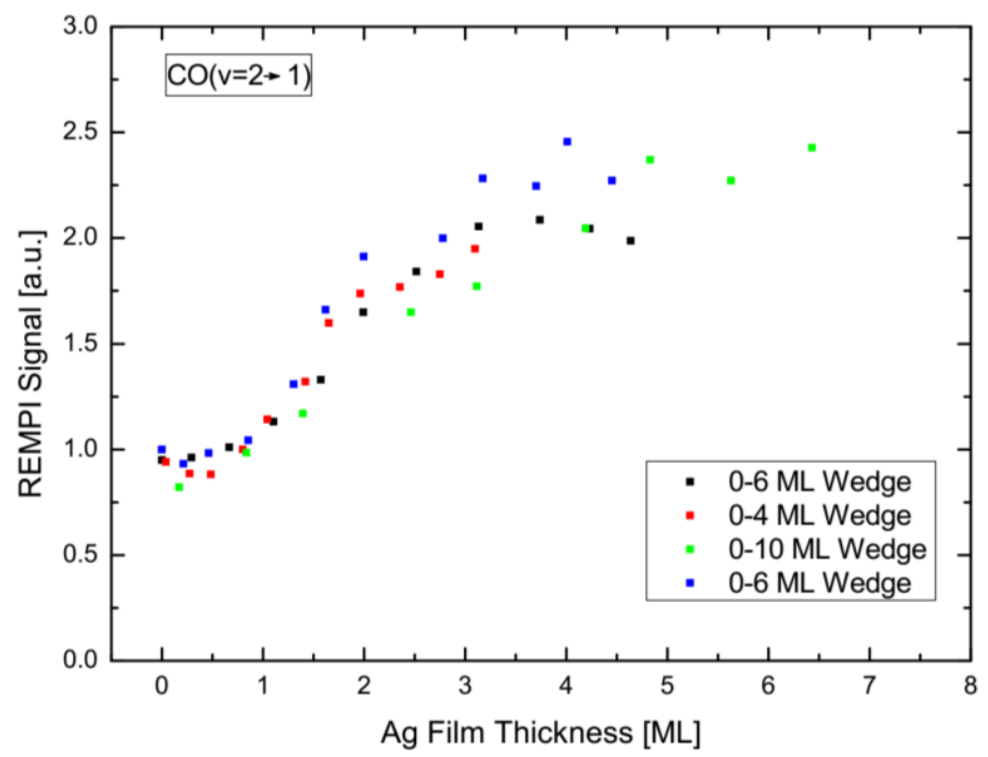

Figure 6.2: REMPI Signal of the $\mathrm{CO}(v=2 \rightarrow 1)$ scattering channel from different wedges.

\subsubsection{Spectra of scattered $\mathrm{CO}$ on $\mathrm{Au}$ and $\mathrm{Ag} / \mathrm{Au}$}

When comparing the REMPI intensity of scattered NO from different surfaces, it was shown that the intensity of a single rotational state can be employed as representative for the complete spectrum. On a molecular level, this interpretation means that the rotational distribution does not change when scattering from different surfaces. Experimentally, it could be shown that the spectra obtained from 
Au or a film of silver differ only by a constant scaling factor. It will be shown in a similar manner that this is true for $\mathrm{CO}$ as well.

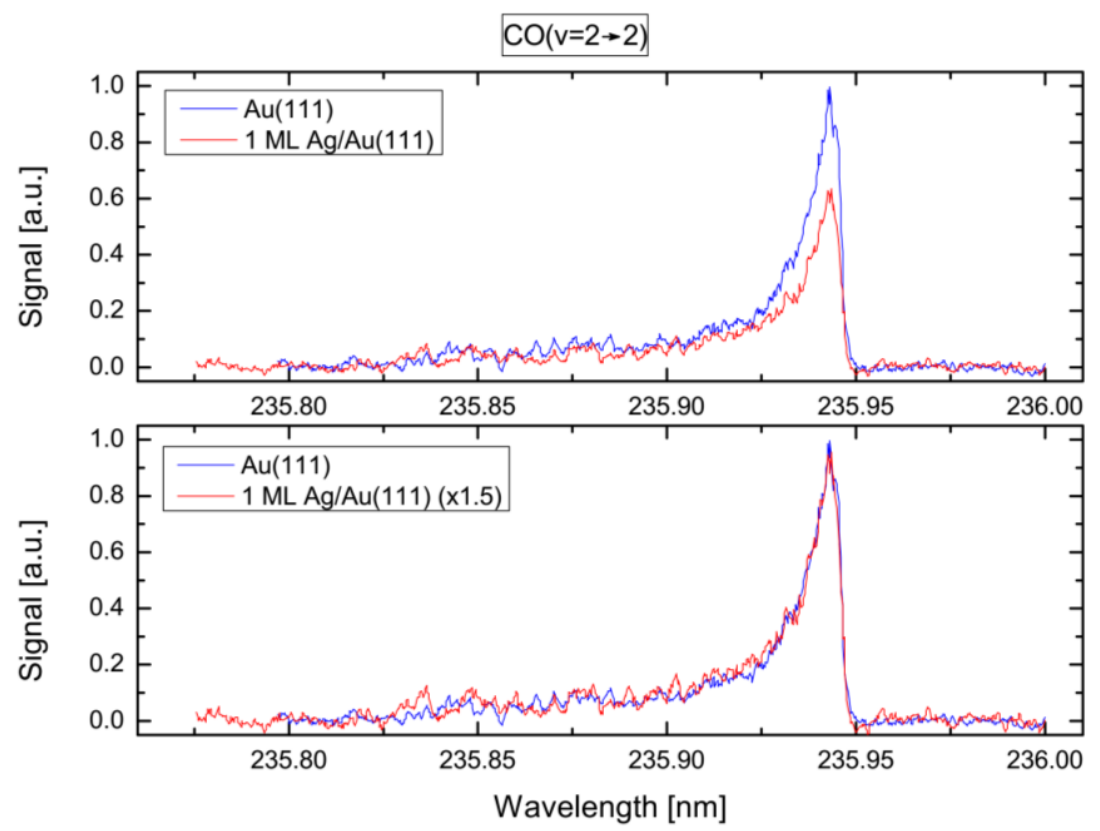

Figure 6.3: $\mathrm{CO}(v=2 \rightarrow 2)$ spectra from $\mathrm{Au}(111)$ and $1 \mathrm{ML} A g / A u(111)$. Top panel: Raw spectrum showing mirroring the signal decrease of the observed vibrational channel on $1 \mathrm{ML} \mathrm{Ag/Au}$ in comparison to Au(111). Bottom panel: Both spectra match when scaling with a constant factor.

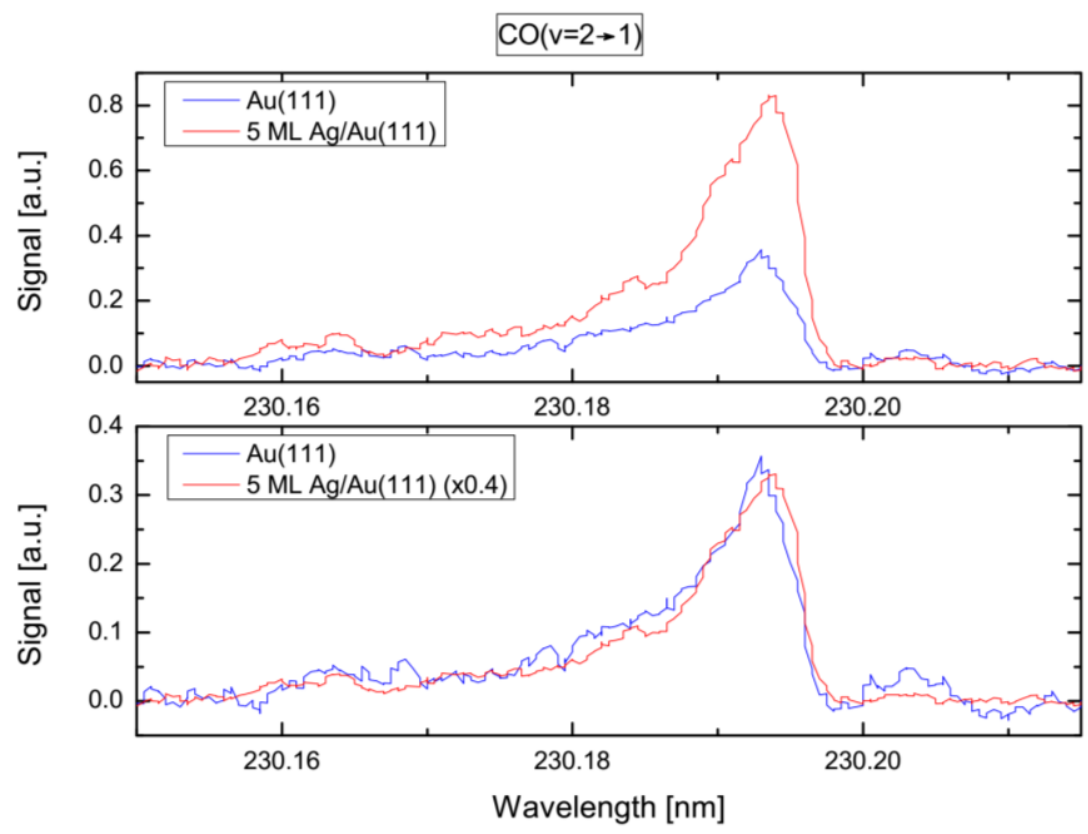

Figure 6.4: $\mathrm{CO}(v=2 \rightarrow 1)$ spectra from $\mathrm{Au}(111)$ and $1 \mathrm{ML} A g / A u(111)$. Top panel: Raw spectrum showing mirroring the signal decrease of the observed vibrational channel on $1 \mathrm{ML} \mathrm{Ag/Au}$ in comparison to Au(111). Bottom panel: Both spectra match when scaling with a constant factor.

Figure 6.3 depicts the REMPI spectra of the $\mathrm{CO}(v=2 \rightarrow 2)$ vibrational band scattered from $\mathrm{Au}(111)$ and $1 \mathrm{ML} \mathrm{Ag} / \mathrm{Au}$. In the employed $\mathrm{B}^{1} \Sigma^{+}\left(v^{\prime}=1\right) \leftarrow \mathrm{X}^{1} \Sigma^{+}\left(v^{\prime \prime}=2\right)$ REMPI transition, $\mathrm{CO}$ is detected via the $Q$ branch with a very limited rotational resolution. The top panel shows the raw spectra after the usual processing step mirroring the absolute signal decrease when scattering from $1 \mathrm{ML} \mathrm{Ag/Au}$. In the 
bottom panel, the spectrum of $\mathrm{CO}$ scattered from the silver surface is scaled by a constant factor which reproduces the spectrum obtained for the reference surface $\mathrm{Au}(111)$. In a similar manner, the $\mathrm{CO}(v=2 \rightarrow 1)$ spectrum is plotted for two different surfaces (Figure 6.4). Here, a film thickness of $5 \mathrm{ML}$ $\mathrm{Ag} / \mathrm{Au}$ is chosen. Apart from the fact that a signal increase is observed on $\mathrm{Ag}(111)$, the spectra from both surfaces differ by a constant multiplication factor. It can be therefore concluded that for $\mathrm{CO}$ (as for NO) comparison of REMPI signals can be reduced to measuring at the peak of the distribution instead of measuring the complete spectrum.

\subsubsection{Corrections for Quantitative Analysis}

As described in 5.1.3 for NO, for a quantitative analysis, it is important to take into account effects that affect the change of the signal intensity independent of a change in relaxation probability. Those effects which occur on a modified surface include different angular distributions, a broadening of the arrival time distribution and the final molecular speed. Suitable correction factors are presented in the following section.

\section{Angular Distributions}
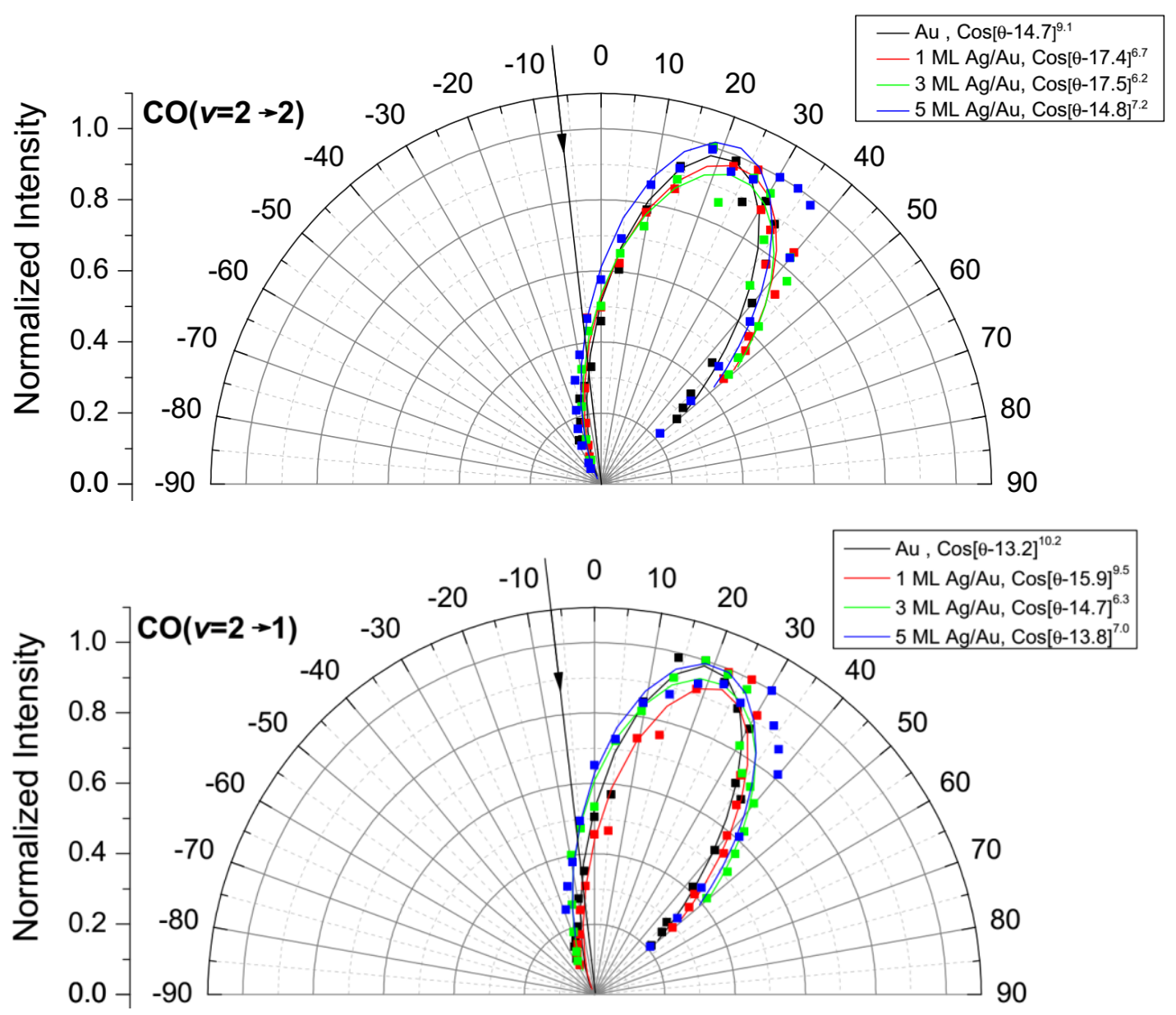

Figure 6.5: Angular distribution of the investigated scattering channels - Top panel: $\mathrm{CO}(v=2 \rightarrow 2)$ scattering channel. Bottom panel: $\mathrm{CO}(v=2 \rightarrow 1)$ scattering channel. All in all, thickness-dependent changes of the distribution are small, values of $n>>1$ for the fitted exponent $n$ of $a \cos ^{n}$-shaped function indicate a direct scattering process. A small black arrow marks the incidence angle $\left(\sim 7^{\circ}\right)$. 
The angular distributions of both experimentally observed $\mathrm{CO}(v=2 \rightarrow 2,1)$ channels are depicted in Figure 6.5. Fits according to a $\operatorname{Cos}\left[\theta-\theta_{0}\right]^{n}$ function with the reflection angle $\theta_{0}$ yielded the exponent $n$ which is a typical measure for the width. This fitting procedure exhibits only minor changes with film thickness; all in all, the obtained exponents are consistent with measurement of a previous study on CO excitation [29]. To obtain suitable correction factors, fitting with a gaussian function $f(\theta)=$ $\operatorname{Exp}\left(\frac{\left(\theta-\theta_{0}\right)^{2}}{2 \sigma^{2}}\right)$ yielded the parameter $\sigma$ from which the FWHM of the distribution could be determined (Figure 6.6, left panel). That FWHM divided by a small adjustment due to relative signal at the measurement position were used to obtain thickness-dependent angular correction factors $C F_{A n g}$. Figure 6.6 (right panel) shows the results, all values are normalized to the reference surface $\mathrm{Au}(111)$ as in the case of NO. Slight broadening is observed, leading to a relative correction of about $20 \%$ and $30 \%$ for the $\mathrm{CO}(v=2 \rightarrow 2)$ and $\mathrm{CO}(v=2 \rightarrow 1)$ respectively.
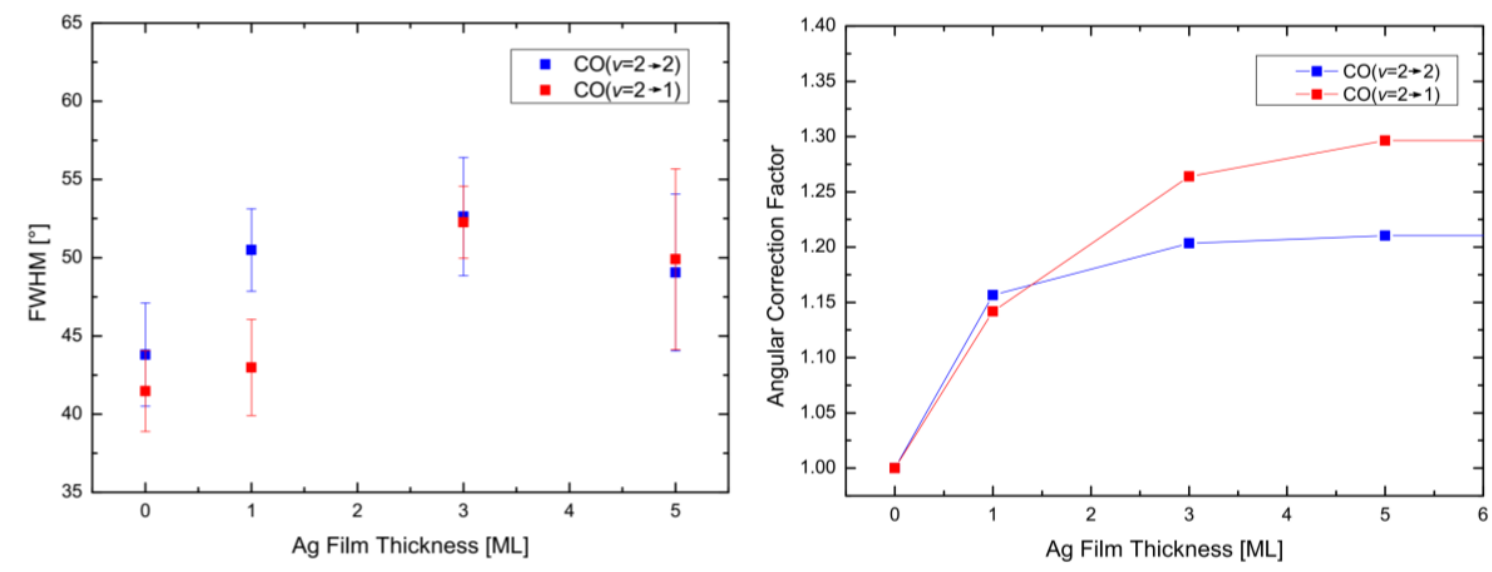

Figure 6.6: Left panel: FWHM of the angular distribution as obtained by a fit with a Gaussian function (see text). Right panel: Correction factors due to the width of the angular distribution.

\section{Arrival time distribution}

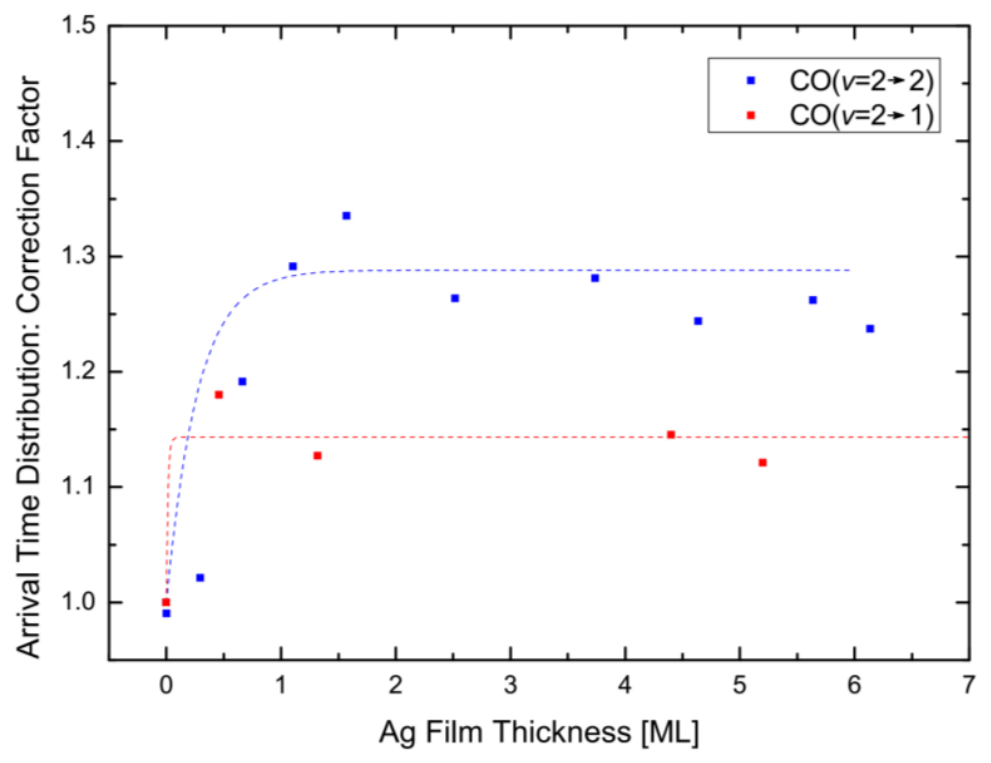

Figure 6.7: Correction factors due to broadening of the arrival time distribution. The data is represented by an analytical function (dashed lines). 
Scattering of $\mathrm{CO}$ from a silver coated surface leads to two changes when measuring the arrival time distribution. The shift of the distribution peak timing to later arrival times is taken care of by adjusting the timing during the measurement. However, as can be seen from Figure 4.8 and Figure 4.9 (section 4.1.3), the width of the distribution is slightly broadening. To determine a correction factor $C F_{T O F}$, the peak-normalized fit functions onto the raw data were numerically integrated and the thicknessdependent values were normalized to the $\mathrm{Au}(111)$ reference. From Figure 6.7, we see that the change of the width is more pronounced in the vibrationally elastic $\mathrm{CO}(v=2 \rightarrow 2)$ channel in comparison to the inelastic. Because the data exhibits a higher scatter, this data is represented by an analytical function describing an increase with exponential decay.

\section{Density to Flux conversion}

The use of REMPI as a density-sensitive detection method affects the signal intensity when measuring molecules of different velocity. Molecules which are scattered from a silver coated surface show a lower translational energy (see Figure 4.10) in comparison to those scattered from $\mathrm{Au}(111)$ and are therefore overestimated. This effect is taken into account by multiplying the signal by the velocity which was obtained in the experiments described in chapter 4. With $\mathrm{Au}(111)$ as a reference, suitable correction factors $C F_{D T F}$ can be gained and are displayed in Figure 6.8.

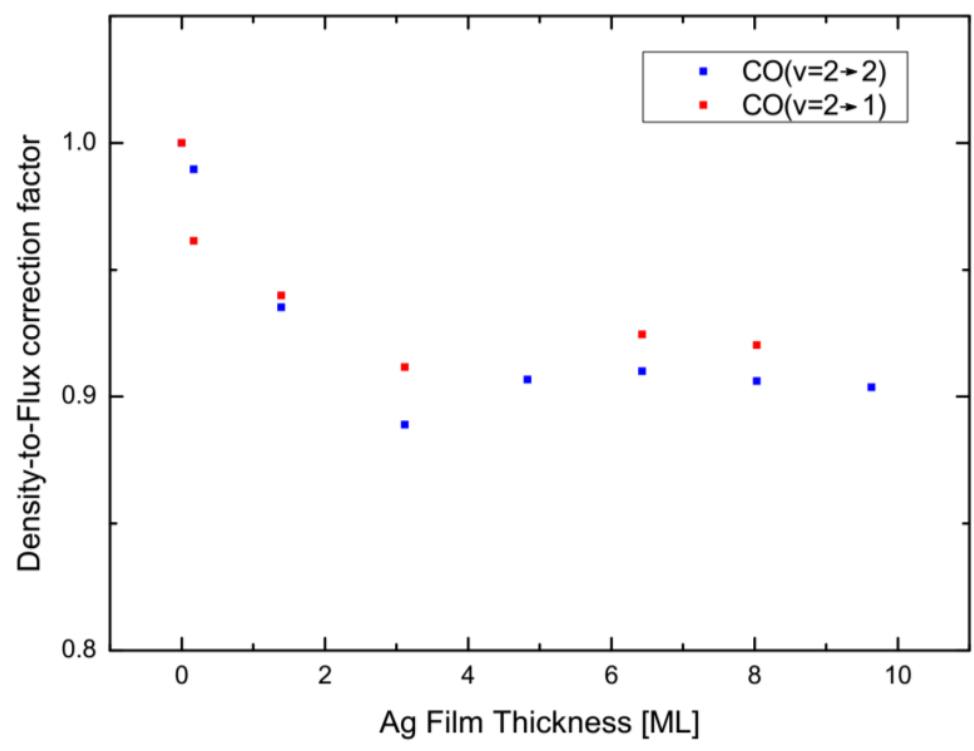

Figure 6.8: Correction factors regarding density-to-flux conversion.

\section{Application of the correction factors}

The effect of the discussed correction factors is best visualized by direct comparison of the raw signal with the finally obtained relaxation probabilities (with reference to $\mathrm{Au}(111)$ ). The data is depicted for the $\mathrm{CO}(v=2 \rightarrow 2)$ and $\mathrm{CO}(v=2 \rightarrow 1)$ channels in Figure 6.9. All in all, the combined corrections lead to a systematic shift of higher signal for increasing film thickness. 

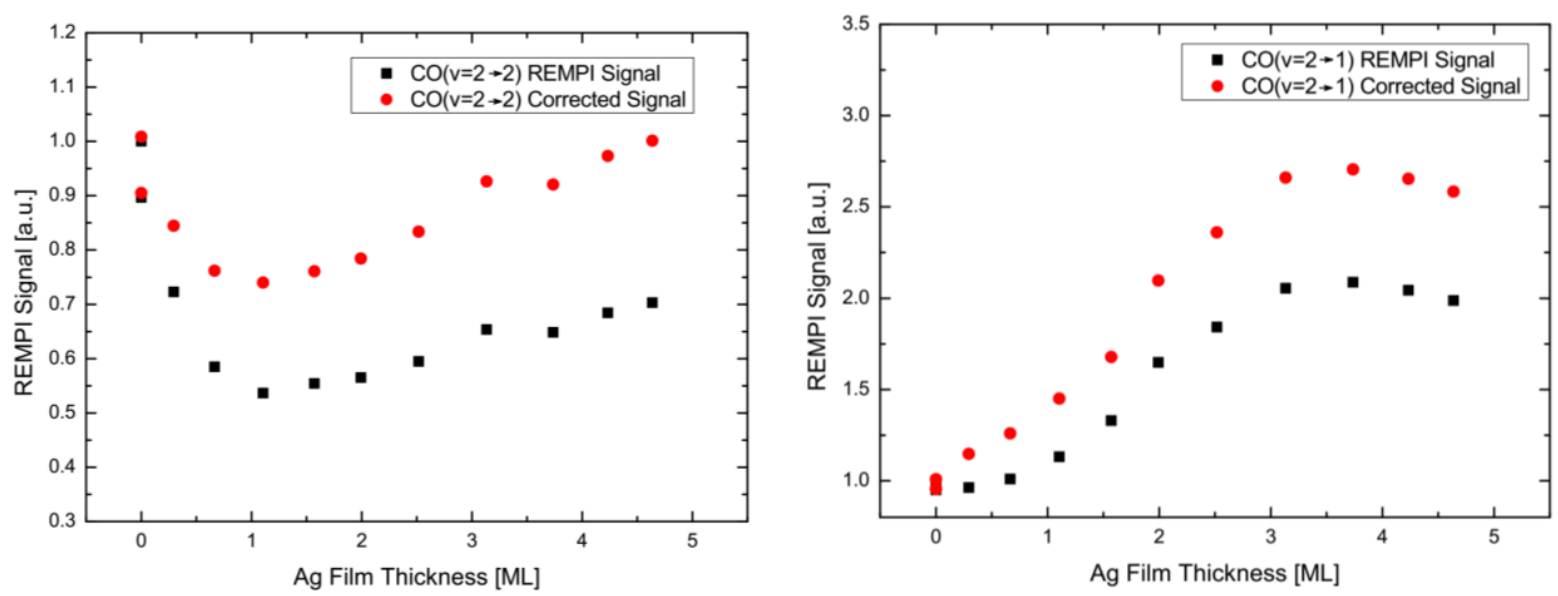

Figure 6.9: Comparison between REMPI signal before and after application of the correction factors. Left panel: $\operatorname{CO}(v=2 \rightarrow 2)$ scattering channel. Right panel: $\mathrm{CO}(v=2 \rightarrow 1)$ scattering channel.

\subsubsection{Determination of Absolute Relaxation Probabilities}

Absolute Relaxation Probabilities of $\mathrm{CO}(v=2)$ on $\mathrm{Au}(111)$

Relaxation probabilities of $\mathrm{CO}(v=2)$ scattered from a $\mathrm{Au}(111)$ surface were determined in an analogous manner as for $\mathrm{NO}(v=2)$. As described in detail in the introduction of this chapter, the analysis is based on the measurement of the REMPI spectra for the vibrational states $\mathrm{CO}(v=2,1)$ which were taken on the peak of the arrival time distribution. Typical spectra for the employed incident energy of $0.64 \mathrm{eV}$ are shown in Figure 6.10.

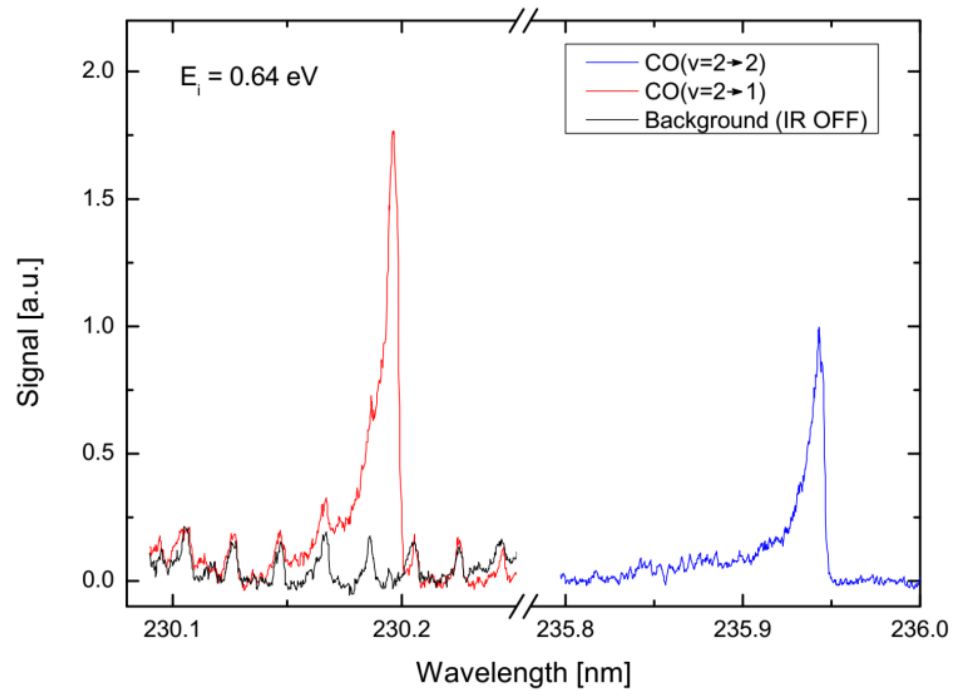

Figure 6.10: Spectra of $\mathrm{CO}(v=2 \rightarrow 2,1)$ after scattering from $\mathrm{Au}$ (111). Depicted are the $Q$ branch of the (1-2) band (blue) and the $Q$ branch of the (1-1) band (red). In the same spectral range of the (1-1) band, some lines of the $O$ branch of the (0-0) band can be found, as seen in a background scan (black) with the IR blocked.

For the detection of $\mathrm{CO}$, the (2+1) REMPI scheme employing the $\mathrm{B}{ }^{1} \Sigma^{+} \leftarrow \mathrm{X}{ }^{1} \Sigma^{+}$transition has strong implication on the quantitative analysis: The Franck-Condon factor of the (1-2) band for the detection 
of $\mathrm{CO}(v=2 \rightarrow 2)$ channel is low $\left(1.41 \cdot 10^{-2}\right)$ in comparison to the (1-1) band (0.972) [105]. As a consequence, after scattering from the surface the number of molecules in the vibrational state $\mathrm{CO}(v=2 \rightarrow 2)$ are substantially increased towards $\mathrm{CO}(v=2 \rightarrow 1)$. The spectra were numerically integrated after the laser power correction was applied. Due to lines of the (0-0) band overlapping with the spectra of (1-1), a background scan with the IR laser blocked was carried out and used for correction. Including the further usual corrections and the Franck-Condon Factors, relaxation probabilities of $\mathrm{CO}(v=2)$ into $v=2,1$ are calculated by neglecting relaxation into $v=0$. As a result, $98( \pm 1) \%$ of the vibrationally excited molecules $\mathrm{CO}(v=2)$ keep the same vibration state, only $2( \pm 1) \%$ lose one vibrational quantum of energy in accordance to previous measurements by Golibrzuch [49].

\section{Absolute Relaxation Probabilities of $\mathrm{CO}(v=2)$ on $\mathrm{Ag} / \mathrm{Au}(111)$}

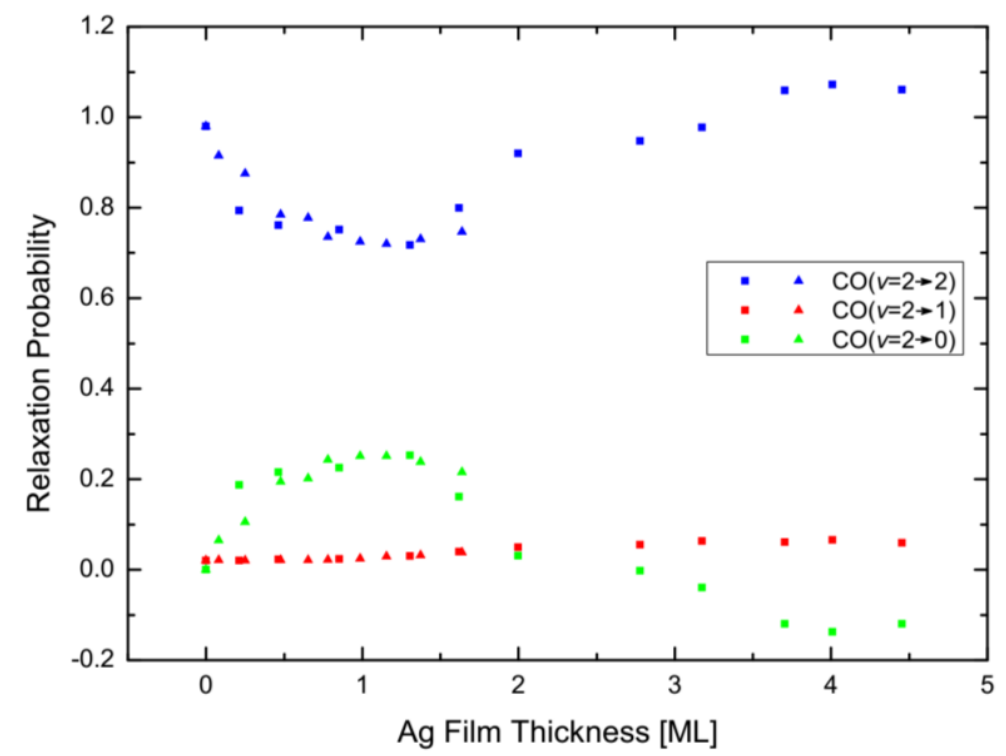

Figure 6.11: Absolute Relaxation Probabilities of $\mathrm{CO}(v=2)$ into $v=2,1,0$. Molecules that not found in $v=2,1$ were assigned to relaxation into the vibrational ground state $v=0$. Two data sets obtained from a 0-5 ML wedge (squares) and a 0-2 ML wedge (triangles) are combined in this plot.

According to equation (5.6), the final result, the thickness-dependent relaxation probabilities were determined for silver films of $\mathrm{Ag} / \mathrm{Au}(111)$ and are shown in Figure 6.11. It is observed that absolute probabilities for the experimentally accessible channels $\operatorname{CO}(v=2,1)$ (blue and red markers) do not add up to $100 \%$ of the molecules. The missing part was calculated and assigned to multi-quantum relaxation into the lowest possible state, $v=0$ (green markers).

Due to the high survival probability of $\mathrm{CO}(v=2)$ on the reference $\mathrm{Au}(111)$ surface, the probability for the $\mathrm{CO}(v=2 \rightarrow 2)$ channel is following the same trend as the previously obtained corrected REMPI signal: In the range of 0-1 ML we see that this channel shows a decrease which is not compensated by an increase in the $\mathrm{CO}(v=2 \rightarrow 1)$ channel. As a consequence, the $\mathrm{CO}(v=2 \rightarrow 0)$ relaxation channel is increasing to a peak of $\sim 30 \%$ at $1 \mathrm{ML} \mathrm{Ag/Au}$. This thickness is a turning point, for higher silver coverages the $\mathrm{CO}(v=2 \rightarrow 2)$ channel increases again, accompanied by a decrease of multi-quantum relaxation channel $\mathrm{CO}(v=2 \rightarrow 0)$. The $\mathrm{CO}(v=2 \rightarrow 1)$ relaxation probability is rising for all thickness but especially between 1-3 ML before reaching a constant level of $\sim 5 \%$. 
It is expected that for a certain film thickness, a relaxation behavior similar to a $\mathrm{Ag}(111)$ bulk surface will be achieved characterized by constant relaxation probabilities. In the TOF experiments (section 4.1.3) it was observed that $\mathrm{CO}$ shows a constant final velocity from $3 \mathrm{ML} \mathrm{Ag/Au}$ on. Can we find a similar behavior for the relaxation on thin films? From the trend of the $\operatorname{CO}(v=2 \rightarrow 2)$ channel, due to experimental limitations it is hard to determine a thickness with constant relaxation behavior. Judging by the raw signals, that thickness is 3-5 ML. However, the $\mathrm{CO}(v=2 \rightarrow 1)$ channel which shows the most pronounced changes with increasing film thickness in comparison to the $\mathrm{Au}(111)$ surface might be the best measure to determine when a $\mathrm{Ag}(111)$ bulk-like behavior is achieved: All signal measurements (compare with Figure 6.2 as well) show a rather constant behavior from $3 \mathrm{ML} \mathrm{Ag/Au}$ which is interpreted as reaching "bulk-like" scattering behavior of $\mathrm{Ag}(111)$.

\subsubsection{Error estimation}

In contrast to NO, the scattering of a molecule with a survival probability close to unity like CO provides a numerical test for the described method to obtain absolute scattering probabilities. After applying corrections for angular distribution, arrival time distribution and density to flux conversion, survival probabilities above $100 \%$ are not physically feasible.

A close inspection of the trends reveals that the changes of the relaxation behavior are rather small in comparison to the experimental limitations of the employed method: Ignoring the inherent measurement error, the increase of the survival probability $\mathrm{CO}(v=2 \rightarrow 2)$ leads to survival probabilities close to or even above unity. Moreover, due to the increase of the $\mathrm{CO}(v=2 \rightarrow 1)$ relaxation with film thickness, negative values for the probability of the $\mathrm{CO}(v=2 \rightarrow 0)$ channel are obtained for films $>3 \mathrm{ML}$. From a conservative estimation, an statistical error bar of about $10 \%$ was calculated for the $\mathrm{CO}(v=2 \rightarrow 2)$ channel which is one way to explain this result.

However, an alternative interpretation of systematical errors is possible: With the assumption that relaxation into $v=0$ is negligible for film thicknesses above $3 \mathrm{ML}$, only two relaxation channels remain. The $\mathrm{CO}(v=2 \rightarrow 1)$ relaxation probability whose error bar is negligible for the employed procedure rises from 2 to max. $5 \%$, as a consequence the other channel, $\mathrm{CO}(v=2 \rightarrow 2)$ is expected to exhibit a probability of $95 \%$ on $\mathrm{Ag} / \mathrm{Au}$. The same value is obtained when determining the survival probability of $\mathrm{CO}(v=2)$ from $5 \mathrm{ML} \mathrm{Ag} / \mathrm{Au}$ with the method as described in the beginning of this section by measuring the spectra. Comparing this result with the previously shown results, it seems that in this thickness range the employed method to determine the absolute scattering probabilities from relative signal leads to a systematic overcompensation of $10 \%$. The correction method taking into account changes of angular distribution, arrival time distribution broadening and density-to-flux conversion is probably the reason for this behavior. As a consequence, the reported probabilities of the $\mathrm{CO}(v=2 \rightarrow 2)$ channel might be systematically too high when the correction factors is applied. The same should apply for the $\mathrm{CO}(v=2 \rightarrow 1)$ channel, since the same procedure was employed, but in this case, the effect on the absolute relaxation probability is rather negligible. 


\subsection{Discussion}

The key observations of the $\mathrm{CO}(v=2)$ relaxation probabilities after scattering from thin films of $\mathrm{Ag} / \mathrm{Au}$ are the following: (1) Scattering from $\mathrm{Au}(111)$ and films with thicknesses above $3 \mathrm{ML} \mathrm{Ag/Au} \mathrm{exhibit} \mathrm{a}$ very similar behavior: Only a very small amount of the molecules (Au: $2 \% ; 3 \mathrm{ML} \mathrm{Ag} / \mathrm{Au}$ : $5 \%$ ) relaxes into the lower vibrational state $v=1$. On silver films, relaxation is increased only by a small degree. (2) A special case is observed for $1 \mathrm{ML} \mathrm{Ag} / \mathrm{Au}$ : Between 0-1 ML, the survival probability $\mathrm{CO}(v=2 \rightarrow 2)$ is decreasing to $70 \%$ while no significant increase occurs in the $\mathrm{CO}(v=2 \rightarrow 1)$ channel. As a consequence, the remaining $25 \%$ are assigned to relaxation via the $\mathrm{CO}(v=2 \rightarrow 0)$ channel. As this remarkable effect of $\mathrm{CO}(v=2)$ scattered from $1 \mathrm{ML} \mathrm{Ag} / \mathrm{Au}$ is not found in the previously described similar system of $\mathrm{NO}(v=2)$, it will be treated as a separate case in contrast to scattering from a bulk-like surface.

First, after assignment of the dominating mechanism, the results will be qualitatively discussed in the context of previous measurements on vibrational energy transfer of $\mathrm{CO}$. The thickness-dependent trends will be directly compared to the $\mathrm{NO}(v=2)$ scattering from thin films.

\subsubsection{Direct Scattering vs. Trapping Desorption}

The discussion of the observed phenomena can be simplified if the scattering mechanism can be clearly assigned. Especially with the behavior at $1 \mathrm{ML} \mathrm{Ag/Au}$ exhibiting strong relaxation into the lowest vibrational state $v=0$, a trapping desorption (TD) mechanism seems to be most probable at first glance. In this case, the vibrationally excited CO molecule would accommodate to the surface and leave in the vibrational ground state. Several aspects speak against this interpretation: The incidence energy $(0.64 \mathrm{eV})$ was chosen to avoid effects like a TD desorption mechanism. An early study about $\mathrm{CO} / \mathrm{Ag}(111)$ scattering including low kinetic energies down to $0.1 \mathrm{eV}$ mentions a TD channel described as "too small to be examined in detail" [86]. In previous $\mathrm{CO}(v=0 \rightarrow 0)$ scattering experiments from a room temperature $\mathrm{Ag}(111)$ crystal carried out in our group, a TD component in the arrival time distribution could be identified for energies about $0.3 \mathrm{eV}$ but not in higher energies like $0.6 \mathrm{eV}$ (See Appendix D). The broadest angular distributions of the observed scattering channels in this work are still narrow to $\cos (\theta)^{6}$ from $3 \mathrm{ML} \mathrm{Ag/Au}$ and peak around the specular angle supporting a direct scattering mechanism.

A comparison of the $\mathrm{CO}(v=2 \rightarrow 2)$ REMPI signal intensity measurements with a gas mixture of higher kinetic energy $\left(1 \% \mathrm{CO} / \mathrm{H}_{2}, 0.92 \mathrm{eV}\right)$ reproduces the signal decrease from $0-1 \mathrm{ML} \mathrm{Ag} / \mathrm{Au}$ providing experimental proof to rule out a TD mechanism in this thickness range with higher relaxation into $v=0$. The survival probability of $\mathrm{CO}(v=2)$ determined for both mixtures is depicted in Figure 6.12. Due to the low amount of $\mathrm{CO}$ in this mixture, the signal-to-noise ratio is lower and a bigger error bar is obtained. It is concluded that relaxation behavior of $\mathrm{CO}(v=2)$ in this work is dominated by a direct scattering mechanism. 


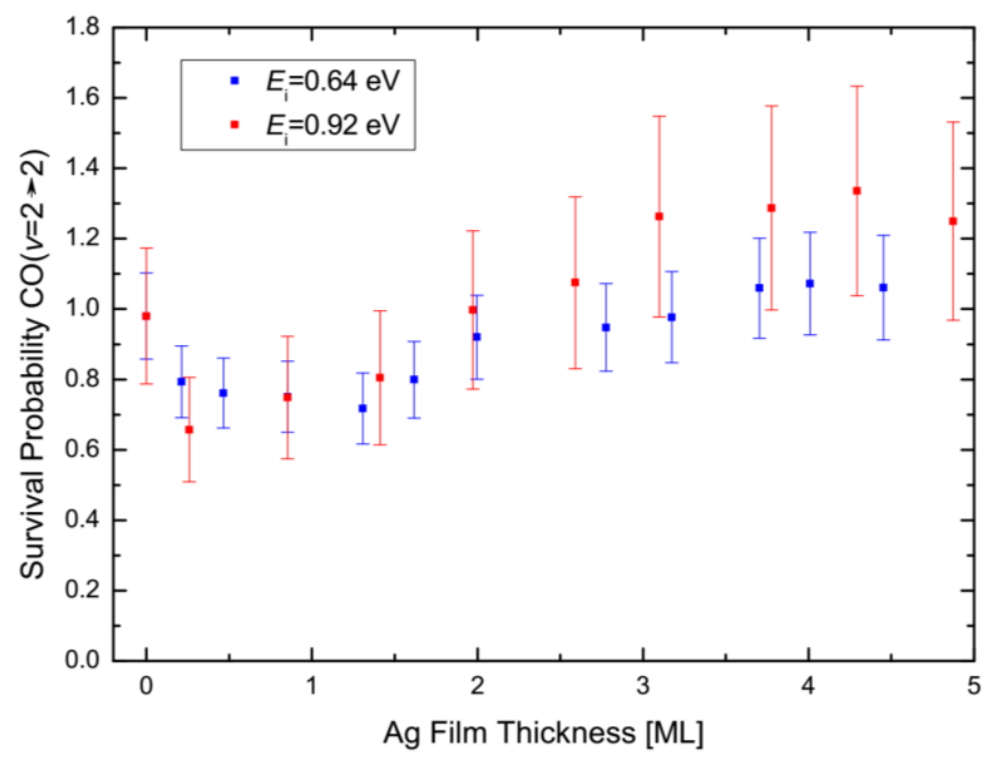

Figure 6.12: Survival probability of $\mathrm{CO}(v=2)$ measured with two mixtures of different incidence energies. Due to the lower amount of $\mathrm{CO}\left(1 \% \mathrm{CO} / \mathrm{H}_{2}\right)$, the signal-to-noise is smaller, leading to higher error bars. Within experimental errors, both measurements are equal. The scattering process is dominated by direct scattering, trapping-desorption can be ruled out to explain the increase in relaxation at $1 \mathrm{ML} \mathrm{Ag/Au}$.

\subsubsection{Vibrational Energy Transfer of $\mathrm{CO}(v=2)$ at Metal Surfaces}

With NO scattering from $\mathrm{Au}(111)$ as a standard, previous studies showed that $\mathrm{CO}$ generally exhibits lower tendency to undergo vibrational excitation or relaxation. $\mathrm{CO}(v=0 \rightarrow 1)$ excitation probabilities after scattering from $\mathrm{Au}(111)$ are reported to be lower by factor of 3-5 in comparison with $\mathrm{NO}(v=0 \rightarrow 1)[40]$. $\mathrm{CO}(v=2)$ relaxation during scattering from the same surface is very inefficient resulting in survival probabilities of $97-99 \%$ [49] $(\mathrm{NO}(v=2) / \mathrm{Au}(111)$ : $76 \%)$. Up to this point, no data was available about vibrational energy transfer of $\mathrm{CO}$ on a silver surface, but as a first working hypothesis, it was assumed that $\mathrm{CO}(v=2)$ relaxation would follow qualitatively the same trend as NO. Since $\mathrm{NO}(v=0 \rightarrow 1)$ excitation is significantly increased on $\mathrm{Ag}(111)$ [30] in comparison to $\mathrm{Au}(111)$ [28] and $\mathrm{NO}(v=2)$ shows substantially stronger relaxation on $\mathrm{Ag} / \mathrm{Au}(111)$ in comparison to $\mathrm{Au}(111)$, consequently an increase of relaxation from a silver coated surface was expected. 


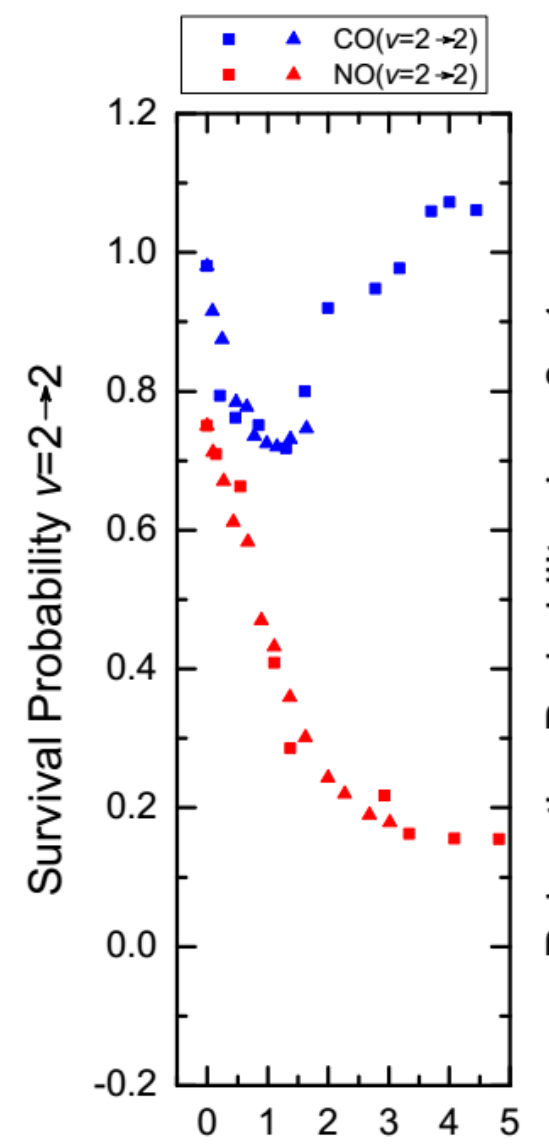

Ag Film Thickness [ML]

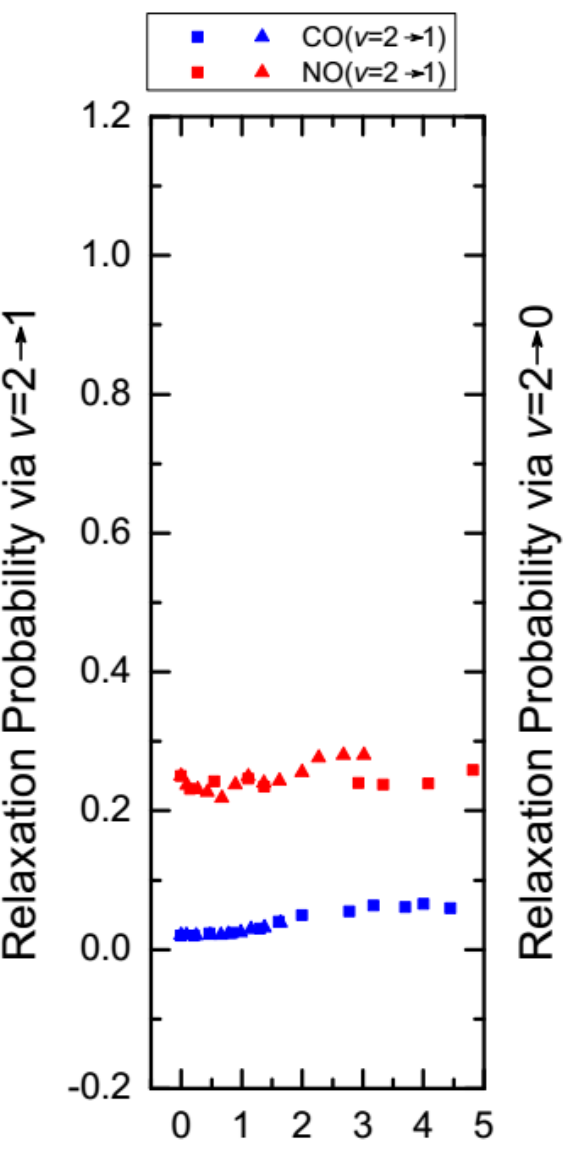

Ag Film Thickness [ML]

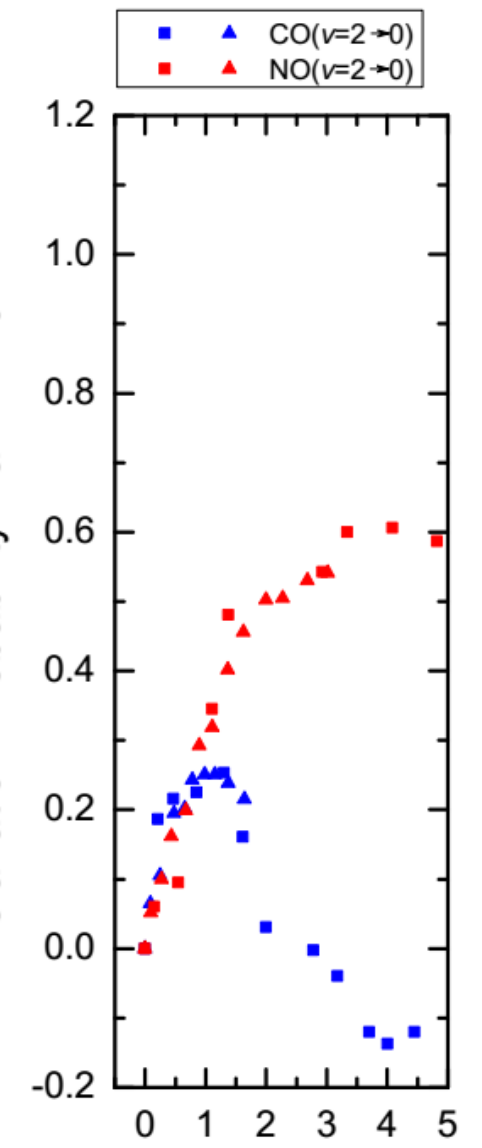

Ag Film Thickness [ML]

Figure 6.13: Vibrational Relaxation of $\mathrm{CO}(v=2)$ and $\mathrm{NO}(v=2)$ on $\mathrm{Ag} / \mathrm{Au}(111)$. Left Panel: Survival Probability vs. Ag Film Thickness. Central Panel: Probability to relax into $v=1$ vs. Film Thickness. Right Panel: Probability to relax into $v=0$ vs. Film Thickness. CO and NO show significantly different behavior.

Therefore, observation (1) of similar survival probabilities on $\mathrm{Au}(111)$ and silver films with $\geq 3 \mathrm{ML}$ $\mathrm{Ag} / \mathrm{Au}(111)$ is an astonishing result. It seems that the properties of a bulk (and bulk-like) surface influence the relaxation only in a negligible manner. Relaxation of $\mathrm{CO}(v=2)$ on metal surfaces is understood as an effect mediated by coupling to electron-hole pairs via the intermediate formation of a $\mathrm{CO}$ anion. The weak tendency to undergo relaxation on both materials in contrast to $\mathrm{NO}(v=2)$ reflects the difference of the molecular electron affinity levels (NO: $0.026 \mathrm{eV}$ [97], CO: $-1.5 \mathrm{eV}$ [106]). Formation of an intermediate $\mathrm{CO}$ anion can only arise with strong image charge stabilization of the anionic potential, possible only at close distance to the surface. A detailed analysis will follow in the coming section.

Observation (2), a strong decrease of the $\mathrm{CO}(v=2)$ survival probability to $70 \%$ at a $1 \mathrm{ML} \mathrm{Ag} / \mathrm{Au}$ surface is a second unpredicted result. Only in a recent experiment of our group, the scattering of highly vibrationally excited $\mathrm{CO}(v=17)$ from $\mathrm{Au}(111)$, a similar low survival probability was found with $60-80 \%$ [103]. The dominant pathway of $\mathrm{CO}(v=2)$ relaxation into the vibrational ground state $v=0$ (up to $25 \%$ probability) attracts attention and is a special feature only found at $1 \mathrm{ML} \mathrm{Ag/Au}$. It will be shown later that this effect cannot be simply explained in the established picture of nonadiabatic relaxation on metal surfaces. It rather seems likely that a different mechanism is the reason for the observed multiquantum relaxation only active when scattering from this special surface. 


\subsubsection{Mechanistic discussion: $\mathrm{CO}(v=2)$ relaxation at bulk (-like) surfaces}

Many mechanistic ideas of vibrational energy transfer in scattering processes due to coupling to surface electron hole pairs were developed to explain excitation and relaxation of NO on a noble metal which is regarded as a model system for those phenomena. In this paragraph, two of those models will be used and accordingly adjusted for the molecule CO. The predicted results will be compared to the experimental data. Because no model does predict the effect at $1 \mathrm{ML} \mathrm{Ag} / \mathrm{Au}$, it will be focused on the observation obtained from bulk(-like) surfaces (i.e. silver films $\geq 3 \mathrm{ML} \mathrm{Ag} / \mathrm{Au}(111)$ ).

Probably one of the most successful approaches describing vibrational energy transfer is a model by Newns [31] which was able to explain the vibrational excitation of $\mathrm{NO}(v=0 \rightarrow 1)$ on $\mathrm{Ag}(111)$ in a quantitative manner. Although this work focusses on the counter-process of vibrational relaxation, many mechanistic features, for example the formation of a transient ion, are believed to the same. In this model, coupling to electron hole pairs is energetically possible for a molecule approaching a surface if its electron affinity level crosses the Fermi level.

Since one of the crucial differences between NO and $\mathrm{CO}$ is the molecular affinity level, it is worth to discuss how the energetic picture changes if this parameter is affected. While accepting an electron leads to a small energy release for NO $(0.026 \mathrm{eV})$, it is energetically unfavored in the case of CO with an electron affinity of $-1.5 \mathrm{eV}$. It is believed that this single parameter can explain the fundamental difference between the relaxation behavior of the two molecules on $\mathrm{Au}$ and $\mathrm{Ag} / \mathrm{Au}(111)$ films of thicknesses $\geq 3 \mathrm{ML}$.

The image charge effect which is fundamental to the stabilization of the molecular affinity level $E_{\mathrm{A}}$ at distance to the metal $z$ is usually described by the simple formula

$$
E_{\mathrm{A}}(z)=E_{\mathrm{A}}(\infty)-\frac{1}{4 \pi \varepsilon_{0}} \frac{e^{2}}{4\left(z-z_{i m}\right)}
$$

where $\varepsilon_{0}$ is the electric constant, $e$ the elementary charge and $z_{i m}$ the position of the image plane. $E_{\mathrm{A}}(\infty)$ is the molecular affinity level at infinite distance which corresponds to the vacuum affinity. With the image plane set to the origin of the coordinate system and ignoring the influence of vibrational oscillations, Figure 6.14 depicts the variation with $z$ of $E_{\mathrm{A}}$ for both molecules $\mathrm{CO}$ and NO.

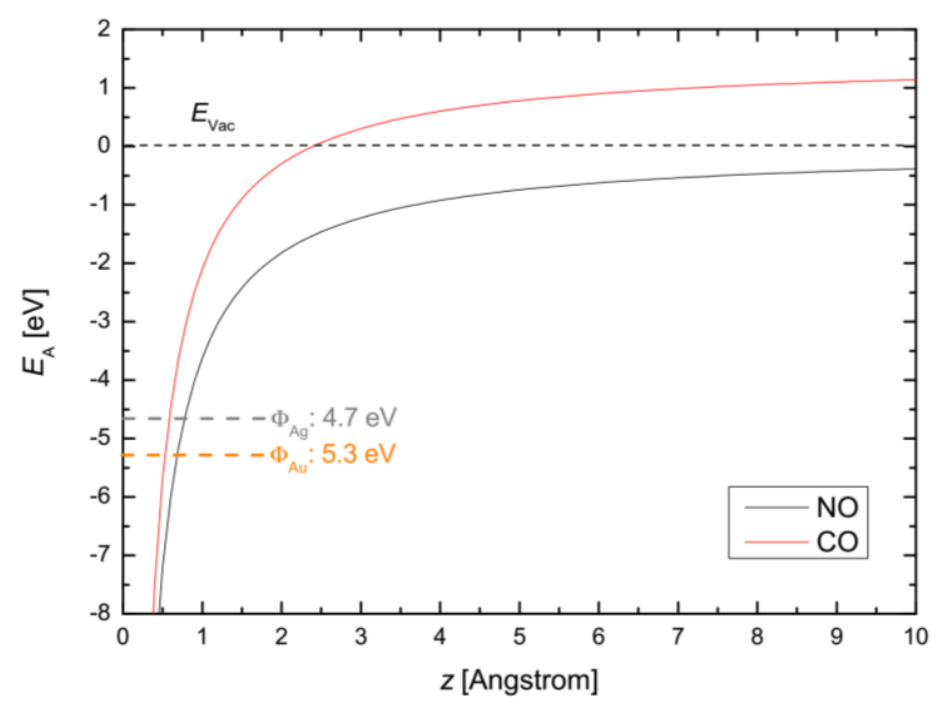

Figure 6.14: Variation of the molecular affinity level with distance to the surface $z$ for NO and CO. The calibration of the $y$ axis is chosen that $E_{\text {vac }}$ is set to the origin $\left(E_{v a c}=0 \mathrm{eV}\right)$. 
In this simple picture, direct comparison shows that in the case of $\mathrm{CO}$, the molecule can only cross the Fermi level at closer distance to the surface. That can explain why $\mathrm{CO}$ shows less vibrational relaxation in comparison to NO because the region for which ion formation can occur is shorter. At the first glance, one could still expected that $\mathrm{CO}$ relaxation will be increased at a silver coated surface. The explanation for increased relaxation of $\mathrm{NO}(v=2)$ on a silver coated surface due to the lowered work function (see 5.2) would be valid for $\mathrm{CO}$ as well. But keeping in mind that repulsion that will be active at small surface distances is neglected, it might be possible that for both $\mathrm{Au}$ and silver coated surfaces $\mathrm{CO}$ cannot approach such small distances. In that case, the image charge stabilization is not effective enough to stabilize the molecular affinity level to form an intermediate ion.

It is possible to extend this picture by taking into account the molecular vibration. Previously, the relaxation of $\mathrm{NO}(v=2)$ scattering from $\mathrm{Ag} / \mathrm{Au}(111)$ was discussed on the basis of a potential energy surface (PES) for the NO/Au(111) system (section 5.2). The employed model developed in the Tully group [98] describes an analytic ground state PES as a combination of two diabatic states (neutral and negative ion NO) which mix depending on the distance to the surface and is based on density functional theory (DFT) calculations. Due to the analytic representation, the adjustment of parameters like the work function or the electron affinity and its effect on the PES can be studied in a qualitative manner. Through comparing the ground state PES from different surfaces by adjusting the work function parameter, it was possible to visualize why a surface with lower work function leads to stronger relaxation of the $\mathrm{NO}(v=2)$ molecule.

In absence of any calculation of a PES for the CO/Au system, this system will be modeled by the same $\mathrm{NO} / \mathrm{Au}(111)$ potential and the choice of suitable parameters. For the case of $\mathrm{CO}$ scattering, the electron affinity is the crucial parameter that needs to be adjusted. As the image charge stabilization is implemented analogously to equation (6.1), by changing the vacuum affinity level $E_{\mathrm{A}}(\infty)$ only an offset is introduced. This parameter adjustment within the existing model seems justified because only the energetic difference of the two adiabatic potentials is shifted.

Other molecular parameters (intramolecular forces are represented by Morse oscillators) to account for a better description of $\mathrm{CO}$ were changed accordingly (details in Appendix $\mathrm{H}$ ), but had only minor influence on the final result. Although this approach will not lead to results which are correct in a quantitative manner, it is expected that trends can be predicted qualitatively. Apart from the electronic properties, the interaction of $\mathrm{NO}$ and $\mathrm{CO}$ with $\mathrm{Au}$ and $\mathrm{Ag}$ will be therefore assumed to be equal. Although those are strong simplifications, it will be shown that the model is consistent with the available relaxation data for vibrationally exicted CO scattered from bulk (-like) surfaces.

A contour plot of the obtained $\mathrm{CO} / \mathrm{Au}(111)$ adiabatic ground state PES with dependency on the bond

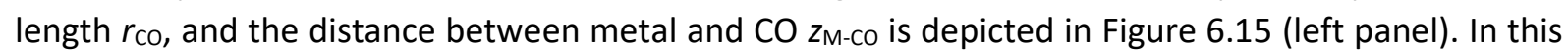
plot, the $\mathrm{CO}$ bond axis is oriented perpendicular to a threefold site of the frozen surface, $\mathrm{C}$ atom down. The black lines define the energetically accessible parameter space for $\mathrm{CO}$ in the vibrational states $v=0-16$. The red line highlights $\mathrm{CO}(v=2)$ marking its inner and outer turning point at $r_{\mathrm{CO}}=1.03 \AA$ and $1.25 \AA$, respectively with zero translational and rotational energy. 

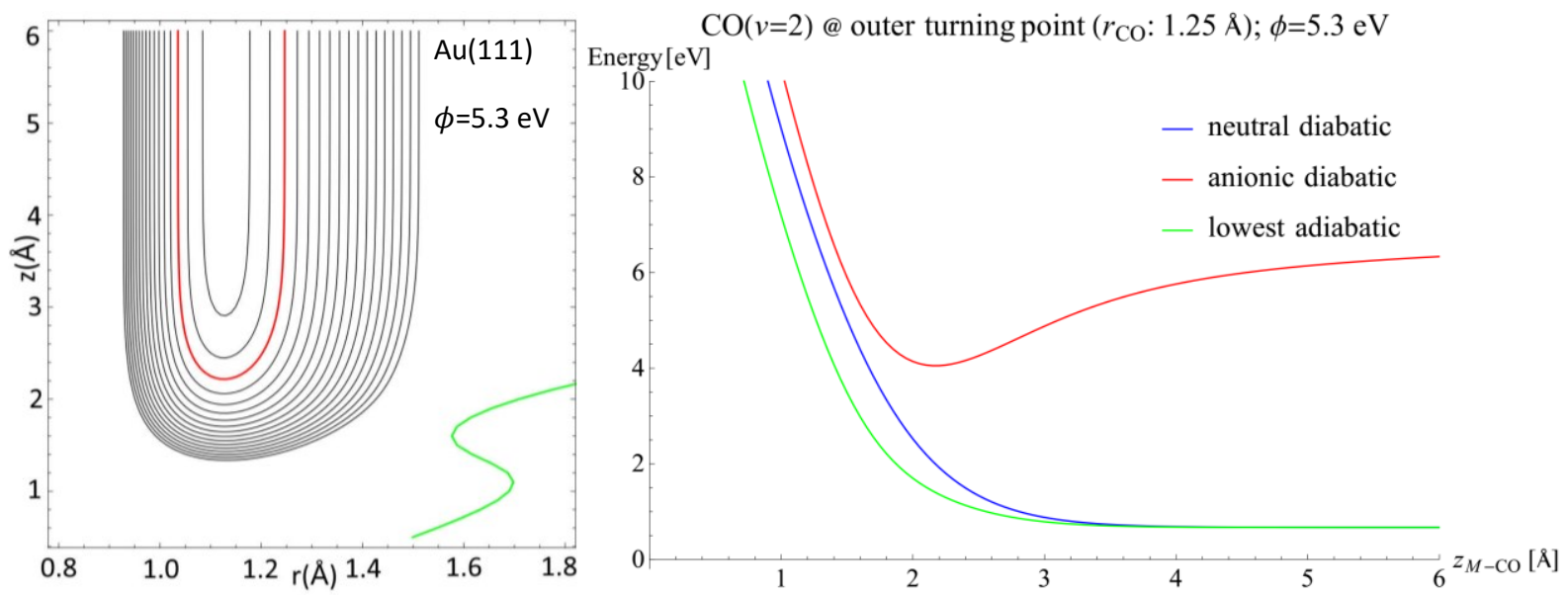

Figure 6.15: Left panel: Contour plot of the CO/Au(111) ground state adiabatic PES (CO perpendicular to threefold site, $C$ down). Dark-blue lines demarcate the regions energetically available to a CO molecule in the states $v=0-16$ with emphasis on $C O(v=$ 2) (red line). The green line marks the region with an ionic character of $50 \%$ of the adiabatic ground state. Right panel: Potential energy curve with bond length fixed to the outer turning point (1.25 $)$ ) at different metal-CO distances. Beside the adiabatic ground state (green) the diabatic states of the neutral and the anionic CO are depicted. Only weak coupling between the two states is possible at distances close to the surface, as there is only negligible difference between the neutral diabatic and the ground-state adiabatic curve.

Efficient vibrational relaxation is possible via intermediate transient ion formation with an electron hopping onto the $\mathrm{CO}$ molecule. This process is favored if the molecule's anionic potential is energetically stabilized towards the neutral potential. The crossing point of both potentials is marked in the contour plot by a green line. This line symbolizes for which parameters the adiabatic ground state potential has $50 \%$ ionic character, vibrational relaxation is therefore expected to be efficient close to this line. It is obvious that the contrast is very strong in comparison to $\mathrm{NO}(v=2) / \mathrm{Au}(111)$ (see Figure 5.20). The large separation between the red and the green line visualizes why relaxation of $\mathrm{CO}(v=2)$ is very inefficient.

The situation is considered from a different perspective in the right panel of Figure 6.15 where three potential energy curves are shown for a $\mathrm{CO}(v=2)$ molecule with fixed bond length at the outer turning point. At this geometry of a stretched molecule the probability density shows a maximum, especially for molecules in high vibrational states. Beside the lowest adiabatic potential whose contour plot is shown in the left panel, the diabatic potentials of $\mathrm{CO}$ and $\mathrm{CO}^{-}$are depicted before mixing. Although it is expected that this is the energetically most favored configuration for electron transfer, no point is found where anionic diabatic potential (red) is crossing the neutral potential (blue). The adiabatic ground state potential (green) is only of weak ionic character close to the surface where it shows slight deviation from the neutral potential.

In this picture, is can be understood why $\mathrm{CO}(v=2)$ relaxation is weak when scattering from a $\mathrm{Au}(111)$ surface. How does the situation change with silver on the surface? Setting the parameter work function from $5.3 \mathrm{eV}(\mathrm{Au}(111))$ to the value of $4.7 \mathrm{eV}(\mathrm{Ag}(111))$, the adjusted PES is shown in Figure 6.16. 

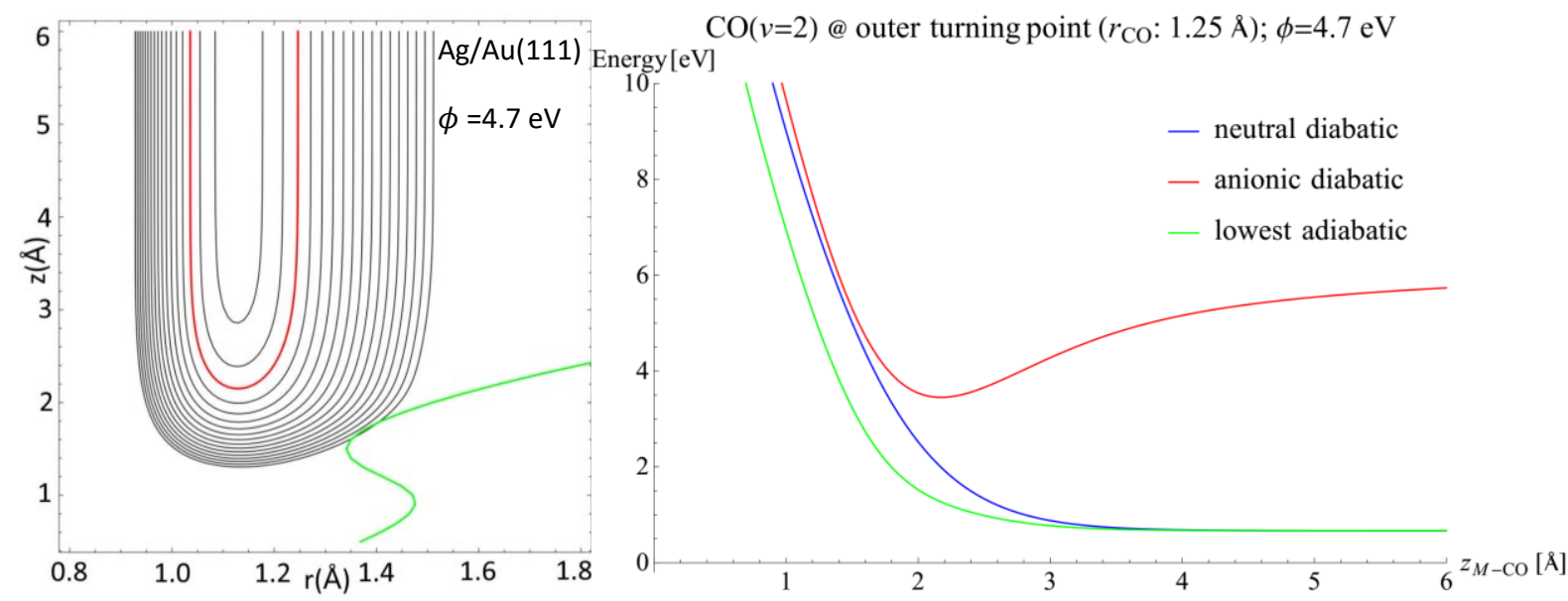

Figure 6.16: Left panel: Similar contour plot as in Figure 6.15 but the work function is lowered to $\Phi=4.7 \mathrm{eV}$. Even with that change, the red line marking the vibrational energy space for $\mathrm{CO}(v=2)$ is far from overlapping with the green line which shows $50 \%$ ionic character of the adiabatic ground state. Right panel: Slice of the contour plot at the vibrational outer turning point. The change in comparison to Au(111) is negligible, therefore surfaces with $>3 \mathrm{MLAg} / \mathrm{Au}$ show still inefficient vibrational relaxation.

In comparison to Figure 6.15 (left panel), the green line marking 50\% ionic character of the adiabatic ground state is closer to the red line defining the parameter space for $\operatorname{CO}(v=2)$. Nevertheless, the distance is still big in comparison to $\mathrm{NO}(v=2) / \mathrm{Au}(111)$ (see Figure 5.20). From the right panel of Figure 6.16, we see that the anionic diabatic state is slightly stabilized for a lower work function, however, the effect seems to be negligible to have a strong influence on the relaxation behavior. Even on a silver surface the formation of the intermediate anion is not stabilized enough to provide a pathway for efficient relaxation.

We might be able to test the validity of the described approach to compare its predictions with one further set of data: scattering of $\mathrm{CO}(v=17)$ from $\mathrm{Au}(111)$ exhibited a survival probability of 60 $80 \%$ [103], which is in the same order of magnitude as observed for the $\mathrm{NO}(v=2) / \mathrm{Au}(111)$ system (76\%). Both contour plots are juxtaposed in Figure 6.17. In a first approximation, in both cases the green line as a measure for efficient vibrational relaxation is at a similar distance to the red line describing the parameters space for the corresponding molecule.

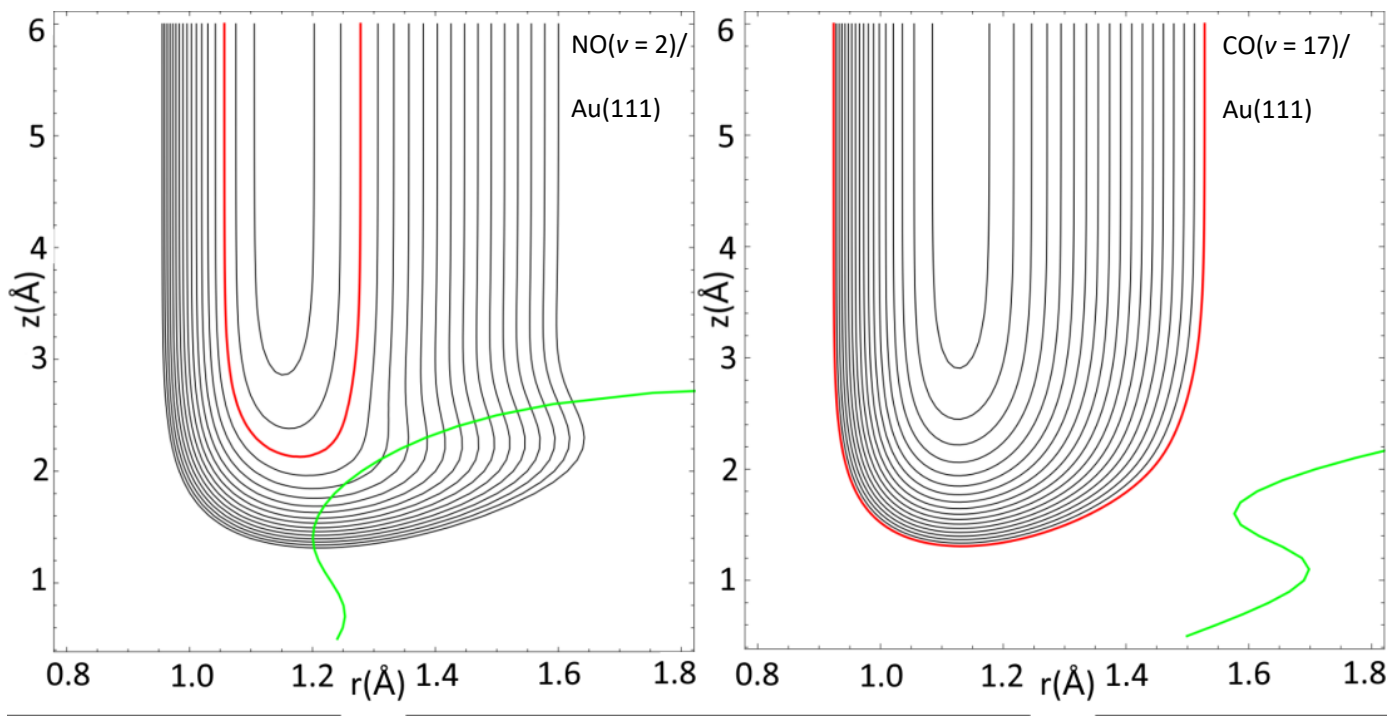

Figure 6.17: Comparison of the contour plots of $N O(v=2)$ and $C O(v=17)$ scattered from Au(111). Qualitatively, in both cases the green and the red line are showing a comparable distance to each other explaining the similar vibrational relaxation probability. 
The suggested approach could serve as a qualitative measure to estimate the strength of strong nonadiabatic vibrational relaxation for a non-reactive system. However, further refinements will be needed to confirm the validity in case of the $\mathrm{CO}$ results. Treating $\mathrm{CO}$ and $\mathrm{NO}$ in a similar manner seems to be the strongest simplification, a PES calculated for $\mathrm{CO} / \mathrm{Au}$ which is in preparation in our group will shed light on this aspect in the future.

\subsection{4 $\mathrm{CO}(v=2)$ at $1 \mathrm{ML} \mathrm{Ag/Au}$ in the context of other relaxation measurements}

Observation (2) of a strong multi-quantum relaxation via $\mathrm{CO}(v=2 \rightarrow 0)$ channel at the thickness of $1 \mathrm{ML} \mathrm{Ag} / \mathrm{Au}$ is a rather unexpected effect. The amount of vibrational energy loss ( $E_{\text {vib }}=0.54 \mathrm{eV}$ ) is too high to be explained by phonon interaction, it is therefore classified as an interaction with electronhole pairs. Why should a silver film of $1 \mathrm{ML}$ thickness exhibit the strongest vibrational relaxation for $\mathrm{CO}(v=2)$ ? In the light of the previous experiments of vibrational relaxation, this effect cannot be predicted: (a) While relaxation of $\mathrm{NO}(v=2)$ showed a continuous increase with thickness between 0 $3 \mathrm{ML} \mathrm{Ag} / \mathrm{Au}$, a singular effect seems to emerge for $\mathrm{CO}(v=2)$ at $1 \mathrm{ML} \mathrm{Ag} / \mathrm{Au}$, a continuous trend is not observed. (b) In the overview plot about relaxation that comprises all measurements of vibrational relaxation from different molecules and surfaces, the results of $\mathrm{CO}(v=2)$ scattering from film between 0-3 ML Ag/Au (Figure 6.18, blue triangles) do not fall into line with in the trend of the results for relaxation of NO in general and CO on bulk(-like) surfaces (dashed line in Figure 6.18).

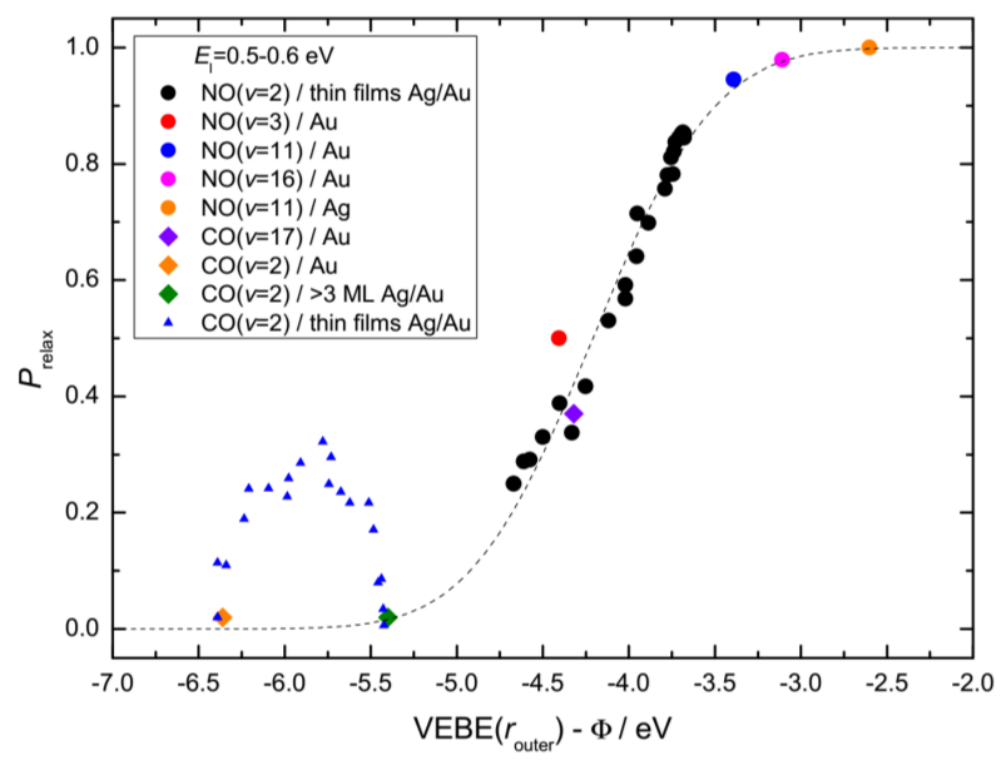

Figure 6.18: Overview about the relaxation probability $P_{\text {Relax }}\left(1-P_{\text {Surv }}\right)$ plotted against the difference between the vertical electron binding energy (VEBE) and the surface work function $\phi$. While all results for relaxation of NO (in general) and CO (on bulk surfaces) seem to follow a trend which can be described by a s-shaped function (dashed line), the results of $C O(v=2)$ relaxation from thin films of 0-3 ML Ag/Au do not blend in.

In a general approach to explain the relaxation of molecules based on the surface properties, it may be possible to identify a different characteristic that emerges only at this single monolayer coverage. The most obvious change that can occur on a bimetallic system are different structural properties. For example, surface strain that arises due to different lattice parameters is known to influence the 
reactivity of surfaces as observed for $\mathrm{CH}_{4}$ dissociation on thin films of $\mathrm{Co} / \mathrm{Cu}(111)$ films [107]. However, due to the negligible lattice mismatch (0.2\%) for $\mathrm{Ag} / \mathrm{Au}(111)$ [12] that effect can be ruled out. The only structural change that is reported for $\mathrm{Ag} / \mathrm{Au}(111)$ is that the well-known herringbone reconstruction of the $\mathrm{Au}(111)$ surface is lifted at $1 \mathrm{ML}$ silver coverage [55]. The unit cell of this $22 \times \sqrt{3}$ reconstruction is however spread out over a number of unit cells of $\mathrm{Au}(111)$, the lifting is therefore a minor effect which does not seem likely to explain the strong increase of relaxation.

Regarding electronic surface properties, the thickness-dependent change of the work function was already discussed in an elaborated manner in the context of $\mathrm{NO}(v=2)$ scattering from $\mathrm{Ag} / \mathrm{Au}(111)$ (section 5.2). It cannot serve as an explanation in the case of $\mathrm{CO}(v=2)$ because it follows a continuous trend with film thickness in contrast to the relaxation behavior. What other electronic effects show a strong dependence on film thickness? One other aspect is the existence of electronic surface states which emerge due to the sudden termination of the bulk at the surface. Energetically, their position is shifting with film thickness closer to the Fermi level [10]. Nevertheless, their relative shift is minimal in comparison to the absolute shift of the Fermi level (due to varying work function).

One effect that was discussed in the context of NO scattering is the shape of the potential energy surface. An indication for such a behavior might be obtained by comparing the experimental temperature programmed desorption (TPD) data. However, TPD studies of CO/Au [99] and $\mathrm{CO} / \mathrm{Ag}$ [108] yield very similar desorption peaks of the CO monolayer at $T_{\mathrm{s}}=52 \mathrm{~K}$ under same experimental conditions. It is conceivable that there is a special effect at $1 \mathrm{ML} \mathrm{Ag/Au}$, however, there is no experimental data available for this metal-metal system.

To conclude, it is not obvious which thickness-dependent effect controls the strong relaxation of $\mathrm{CO}(v=2)$ at $1 \mathrm{ML} \mathrm{Ag} / \mathrm{Au}$, however, it is an intriguing finding that the single monolayer itself seems to cause this behavior. This might be the first finding of such an effect for vibrational relaxation, however, there is a number of studies about the properties of bimetallic layers which will be discussed in the next section.

\subsubsection{A special single layer effect for $\mathrm{CO}$ on $\mathrm{Ag} / \mathrm{Au}$ ?}

The high relaxation probability of $\mathrm{CO}(v=2)$ scattering from $1 \mathrm{ML} \mathrm{Ag/Au} \mathrm{might} \mathrm{be} \mathrm{assigned} \mathrm{to} \mathrm{an} \mathrm{effect}$ that is characteristic for a single layer metallic film. A striking feature of single metal layers supported by noble or transition metals is their increased binding energy [109]. A representative study by Rodriguez and Goodman examined this effect for a single layer $\mathrm{Cu}$ and $\mathrm{Pd}$ bound to $\mathrm{Re}(0001)$ [72]. TPD measurements were carried out on those bimetallic system to determine the binding strength of the metal-metal bond. At film with more than $1 \mathrm{ML}$ coverage, a peak due to multilayer desorption was observed at a lower temperature before the single layer peak desorbed at a relatively higher temperature. The desorption energy of this high temperature peak reflects the bond energy between adatom layer and substrate. Moreover, it was found that the bond strength is influenced by electronic perturbations from the supporting material: X-ray photoelectron spectroscopy (XPS) measurements of the core level binding energy for the overlayer material revealed a shift in comparison to the value measured from a bulk sample [73]. Strong core level shifts were explained by significant electronic interactions between adlayer and substrate and correlated to a higher binding energy of the adlayer. It is therefore not surprising that those electronic influences control the chemical properties of an adatom layer as was quantified by measuring the TPD desorption peak of $\mathrm{CO}$ at a bimetallic surface. 
While it was concluded that strong electron donor-electron acceptor interactions deactivate palladium and nickel adlayers towards $\mathrm{CO}$ chemisorption, adlayers of $\mathrm{Cu}$ were activated. A theoretical model by Ruban et al. explained the effects by shifts of the $d$-band center of the overlayer and provided ab initio calculation for several metals [76].

Could similar effects be present for the system of $\mathrm{Ag} / \mathrm{Au}$ as well? Bonding energy and the electronic effects could provide indications for such a behavior. TPD measurements to estimate the bond energy of $\mathrm{Ag} / \mathrm{Au}$ are not possible due to the miscibility and formation of an alloy at high temperatures. But judging from reported bond dissociation energies [110], the Ag-Au bond (2.10 eV) is significantly stronger in comparison to the Ag-Ag bond $(1.69 \mathrm{eV})$, a trend that might be transferrable when comparing metal layers. The estimation of electronic effects between Ag and the Au substrate is not straightforward. From the differences in the bulk electronegativity (Ag: 1.93; Au: 2.4, Pauling scale) [110] a polarized Ag-Au bond is suggested with partially positive charge at the Ag overlayer. But taking into account that a surface atom is in an anisotropic environment, large variations could arise through orbital rehybridization which lead to differences between bulk and surface electronegativity [13]. Experimental data is only available for the similar system of $1 \mathrm{ML} \mathrm{Cu/Au}$ which exhibits a lower XPS binding energy in comparison to a thicker coverage [74] indicating that Au acts as an electron donor, although the opposite trend would be expected from the bulk electronegativity (Cu: 1.90; Au: 2.4, Pauling scale) [110]. Maybe an indication can be obtained from investigations on Ag-Au alloys. From XPS measurements it was concluded that the Au d-orbital charge transfer to Ag is stronger than the Ag $s$-orbital charge transfer to Au which would be consistent with the idea of $\mathrm{Au}$ as a net electron donor [111]. It is possible that similar effects could exist on the Ag-Au bilayer system as well.

Although it seems conceivable that the heteronuclear bonding nature of the bimetallic surface of $1 \mathrm{ML}$ $\mathrm{Ag} / \mathrm{Au}$ exhibits singular characteristics, it is not clear which property leads specifically to the strong increase of vibrational relaxation for $\mathrm{CO}(v=2)$. The only existing study about increased nonadiabatic vibrational transfer without invoking the effect of work function examined $\mathrm{N}_{2}(v=0 \rightarrow 1)$ excitation. While no excitation was found on $\mathrm{Au}(111)$, excitation probabilities of $10^{-4}$ to $10^{-3}$ were measured when scattering from $\mathrm{Pt}(111)$ [39] although $\mathrm{N}_{2}$ exhibits an even lower electron affinity $\left(E_{\mathrm{A}}=-2.3 \mathrm{eV}\right)$ than $\mathrm{CO}$ $\left(E_{\mathrm{A}}=-1.5 \mathrm{eV}\right)$. This difference was explained by the position of the $d$-orbitals which are close the Fermi level for Pt(111) but not for $\mathrm{Au}(111)$. It might be possible that the center of the $d$-orbitals is shifted towards the Fermi level for a single layer $\mathrm{Ag} / \mathrm{Au}$ which causes the increased relaxation. The previously mentioned model predicts a shift for $\mathrm{Ag} / \mathrm{Au}$ towards the Fermi level and a shift in the opposite direction for Au/Ag [76]. Nevertheless, the expected shift of the d-orbitals might be too small to have an effect since the $d$-orbitals of Au and Ag are at energetic distance of $>1 \mathrm{eV}$ below the Fermi level. Alternatively, the polarized nature of the metal-metal bonding could lead to a higher local density of electrons at the single silver layer or higher stabilization of the anionic potential, both effects that might facilitate the transient ion formation and increase the relaxation probability.

However, it is not possible to draw a final conclusion at this point. As the first reported single layer effect for vibrational relaxation and only scarce data about the $\mathrm{Ag} / \mathrm{Au}$ bilayer system, further studies will be necessary to investigate the observed effects. It is hoped that our findings can also stimulate theoretical efforts helping to understand the experimental results. 


\section{Conclusion \& Outlook}

To conclude, we studied thickness-dependent translational energy transfer during scattering of $\mathrm{NO}(v=2,0)$ and $\mathrm{CO}(v=2)$ from ultrathin metallic films of $\mathrm{Ag} / \mathrm{Au}(111)$. For both molecules, time-offlight experiments at different film thicknesses showed that the final mean translational energy of the vibrationally elastic and inelastic scattering channels decreases in the range of 0-3 ML Ag/Au. For higher thicknesses, constant behavior is observed which is interpreted as a bulk-like behavior. Results for $\mathrm{Au}(111)$ and $\geq 3 \mathrm{ML} \mathrm{Ag} / \mathrm{Au}$ are consistent with the Baule-Limit assuming head-on collision with a single surface atom. The trend between 0 and $3 \mathrm{ML} \mathrm{Ag/Au}$ can be modeled by assuming a gradual change of the effective surface mass that is experienced by the scattered molecule. A participation of up to two subsurface layers in the scattering process is suggested. The thickness dependence of the final kinetic energies exhibits the same trend for all investigated molecules and scattering channels and is purely dominated by mechanical properties of the surface.

Furthermore, the vibrational inelasticity of $\mathrm{NO}(v=2)$ and $\mathrm{CO}(v=2)$ scattering from $\mathrm{Ag} / \mathrm{Au}(111)$ was investigated and thickness-dependent vibrational relaxation probabilities of $\mathrm{NO}(v=2)$ and $\mathrm{CO}(v=2)$ were measured. Both molecules exhibit a significantly different trend. The relaxation probability of $\mathrm{NO}(v=2)$ increases gradually between thicknesses of $0-3 \mathrm{ML} \mathrm{Ag} / \mathrm{Au}$ and strongly suggests that the change of the work function influences the amount of relaxation. In contrast, the relaxation probability of $\mathrm{CO}(v=2)$ at $\mathrm{Ag} / \mathrm{Au}(111)$ is of similar order of magnitude for $\mathrm{Au}(111)$ and $\geq 3 \mathrm{ML} \mathrm{Ag} / \mathrm{Au}$ but shows a maximum for $1 \mathrm{ML} \mathrm{Ag/Au}$.

The results of the present work extend our understanding of scattering processes for surfaces that exhibit higher complexity than crystalline surfaces of a single material.

One the one hand, the trend of translational inelasticity over the first single layers shows that subsurface layers can influence the scattering process. That might especially affect the energy loss of molecules that collide with metallic nanoparticles. While the role of electronic effects of metal-oxide supported nanoparticles is well-established in the field of catalysis [112], the effective mass of such transition metals could additionally be influenced due to the fact that they are supported by a material of light mass $\left(\mathrm{Al}_{2} \mathrm{O}_{3}, \mathrm{SiO}_{2}\right)$. As a consequence, scattering molecules could lose a higher amount of kinetic energy on supported nanoparticles in comparison to bulk surfaces which might increase the tendency to trap and undergo chemical reactions. We hope that our findings might improve models that simulate the scattering processes. Ab initio molecular dynamics (AIMD) calculations for CO scattered from $\mathrm{Ag} / \mathrm{Au}$ at different film thickness are ongoing work in our group. Initial results failed to reproduce the experimental results which could indicate that the modeling of a process that is believed to be well-understood like the translational energy transfer might still offer a few surprises when the scattering target is a more complex surface.

On the other hand, most of the data about molecular vibrational relaxation can be understood in a simple model predicting the relaxation probability based on two parameters, the vertical electron binding energy (VEBE) and the surface work function $\phi$. Although an analogous model for a different nonadiabatic effect, the anion emission of electrons, has been proposed previously, simple models always raise the doubt if they can be applied to several systems. Is it possible to predict vibrational relaxation for surfaces with a higher density of states at the Fermi level? A surface like Pt(111) which exhibited a higher probability for $\mathrm{N}_{2}(v=0 \rightarrow 1)$ vibrational excitation than $\mathrm{Au}(111)$ might as well exhibit higher relaxation probability, although the model predicts a lower value due to the high surface work function. 
Furthermore, in all the investigated processes, molecular dissociation does not play a significant role. Can the same model be applied to a surface like $\mathrm{Cu}(111)$ which is known to have a lower dissociation barrier for NO?

The investigation of those aspects could help to answer the general question which role the amount of nonadiabatic vibrational relaxation plays for a reactive gas-surface system. There are many systems for which vibrational excitation significantly increases the dissociation probability of an incoming molecule described by the vibrational efficacy [113]. One the one hand, one could image that vibrational relaxation is an energy loss channel for a molecule which reduces the reactivity of such a gas-surface system. On the other hand, the parameters which predict a high relaxation probability, like a low work function and high molecular tendency to accept an electron, facilitate electron transfer reactions. From that point of view, a strong nonadiabatic coupling could increase the reactivity of a molecule-surface system.

The high relaxation probability for $\mathrm{CO}(v=2)$ on $1 \mathrm{ML} \mathrm{Ag} / \mathrm{Au}$ is an effect that is still not well-understood. However, its observation tells us that that there may be nonadiabatic effects that are characteristic for a nanoscale structures like bimetallic films or even nanoparticles used in modern catalytic processes. For a detailed understanding and modeling of fundamental energy transfer processes on such structures, it would be of interest to focus future studies on surfaces structurally closer to "real world" catalysts with the aim to obtain the knowledge to design energy conversion on an atomic scale. 


\section{Appendix}

\section{A Performance curves of the Evaporator}

This section shows a full set of performance curves of the home-built evaporator.
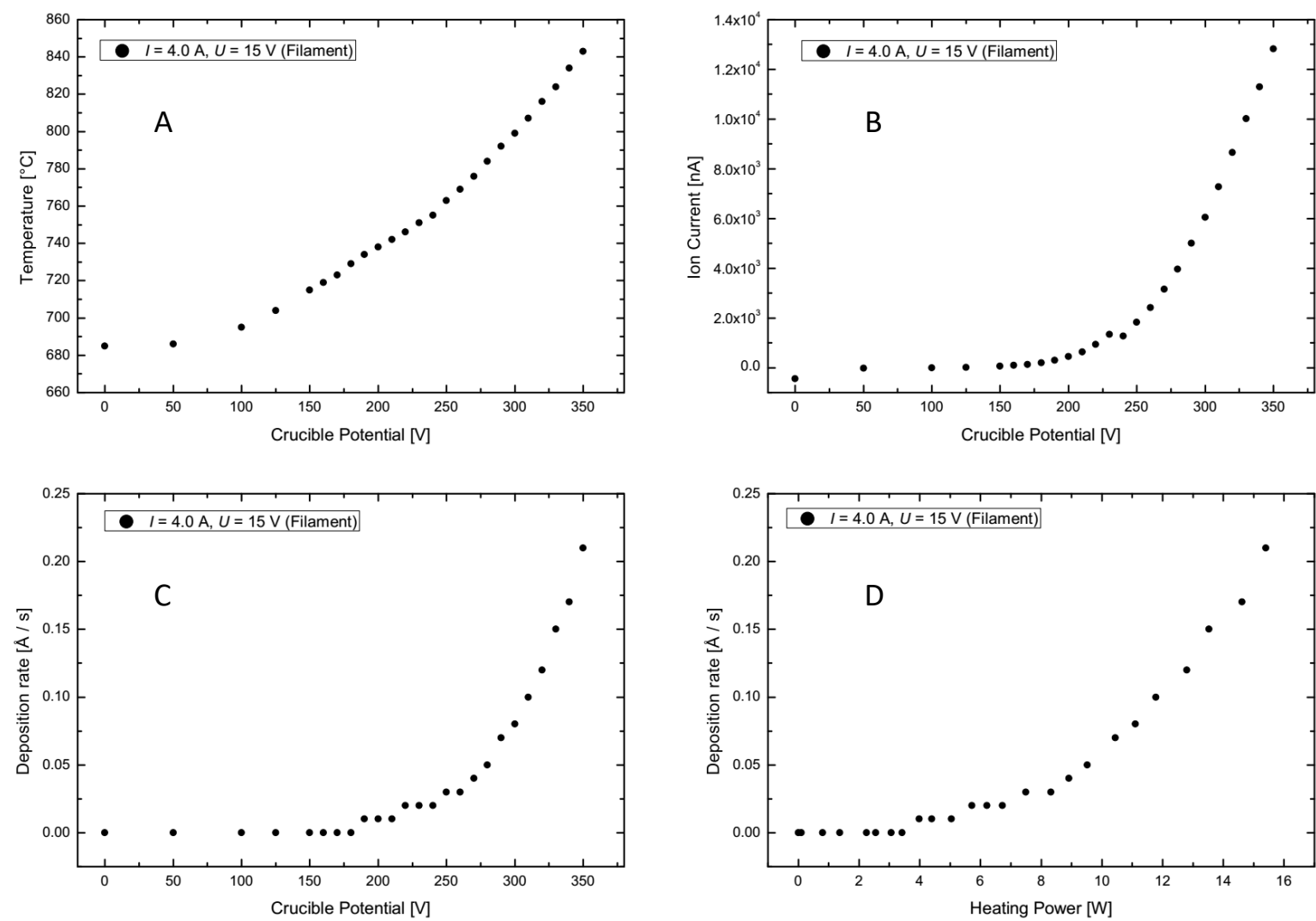

Figure 8.1: Typical set of performance curves. The filament's current was set to $4 \mathrm{~A}$ and the potential at the crucible was increased. A) Trend of the temperature with increasing crucible voltage. B) lon current measured at the final aperture with increasing crucible potential. C) (uncalibrated) deposition rate measured by the quartz crystal microbalance plotted against the crucible potential. D) Deposition rate (from C) plotted against the heating power. 


\section{B Analysis of the TOF data}

This part of the appendix describes how the measured arrival time distributions were fitted using the notation of Ref. [89].

In the present work, REMPI is used for the state-selective detection of molecules. REMPI is a densitysensitive technique that means that the observed ion signal is proportional to the number density in the ionization region. In a TOF experiment, the time-dependent density $S(t) d t$ of molecules over a differential time element is acquired. The raw TOF data is first converted into flux $I(t) d t$ by multiplication with the time-dependent velocity $v(t)$ of the molecules.

$$
I(t) d t=S(t) v(t) d t
$$

$I(t) d t$ is related to the velocity-dependent flux $P(v) d v$ by

$$
P(v) d v=I(t) d t=S(t) v(t) d t
$$

which leads to

$$
P(v)\left|\frac{d v}{d t}\right|=S(t) v
$$

Now, the Jacobian for the correct transformation can be applied for the conversion from time space into velocity space

$$
\left|\frac{d v}{d t}\right|=\frac{l}{t^{2}}=\frac{v^{2}}{l}
$$

with velocity $v$ and flight distance $l$. Finally, we obtain

$$
\begin{gathered}
P(v) \frac{l}{t^{2}}=S(t) v \\
\Leftrightarrow S(t)=P(v) \cdot \frac{1}{v} \cdot \frac{l}{t^{2}}
\end{gathered}
$$

when applying the density-to-flux conversion once. For the tagging experiments including two densitydependent steps (IR excitation and REMPI detection), two conversion steps are applied which leads to

$$
S(t)=P(v) \cdot \frac{1}{v^{2}} \cdot \frac{l}{t^{2}}
$$

The velocity-dependent flux $P(v)$ is described by a floating Maxwell-Boltzmann distribution $f(v)$ multiplied by the velocity $v$, resulting in

$$
P(v)=f(v) v=A v^{3} \exp \left(-\left(\frac{v-v_{0}}{\alpha}\right)^{2}\right)
$$

where $A$ is an amplitude factor, $v_{0}$ the center velocity of the distribution and $\alpha$ a width parameter. The fitting function for $S(t)$ is obtained by replacing $P(v)$ of (8.6) by its definition in (8.8) and use $=\frac{l}{t}$ :

$$
S(t)=A \cdot\left(\frac{t}{l}\right)^{2} \cdot\left(\frac{l}{t^{2}}\right) \cdot\left(\frac{l}{t}\right)^{3} \exp \left(-\left(\frac{\frac{l}{t}-v_{0}}{\alpha}\right)^{2}\right)
$$


After fitting the TOF data, $P(v)$ is obtained and can be converted into energy space. With the relation $P(E) d E=P(v) d v$ and $E=\frac{1}{2} m v^{2}$, the Jacobian $\frac{d E}{d v}=m v=\sqrt{2 m E}$ is employed to yield

$$
P(E) d E=\frac{P(v)}{m v}
$$

\section{Comparison $\mathrm{NO}(v=2 \rightarrow 2)$ and $\mathrm{NO}(v=0 \rightarrow 0)$ on $\mathrm{Au}$ and $\mathrm{Ag} / \mathrm{Au}$ with $\mathrm{NO}(v=0 \rightarrow 0)$ from bulk $\mathrm{Au}(111), \mathrm{Ag}(111)$}

$\mathrm{Au}$ (bulk) and $\mathrm{Ag}$ (film)

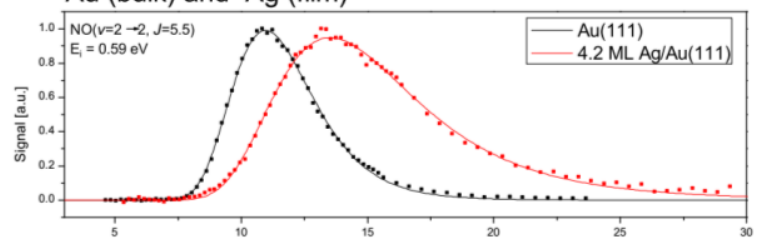

Au (bulk) and $\mathrm{Ag}$ (film)

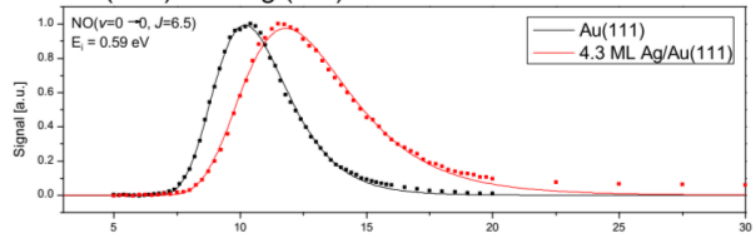

$\mathrm{Au}$ (bulk) and $\mathrm{Ag}$ (bulk) TOF [us]

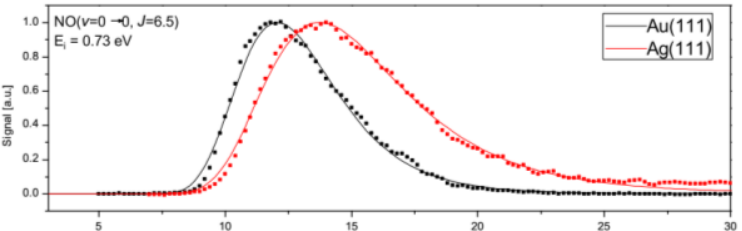

$\mathrm{Au}$ (bulk) and $\mathrm{Ag}$ (bulk) ToF [us]

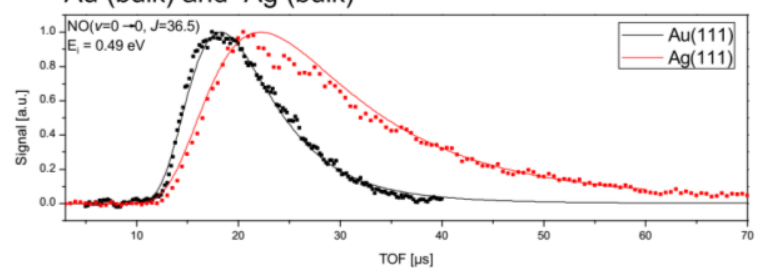

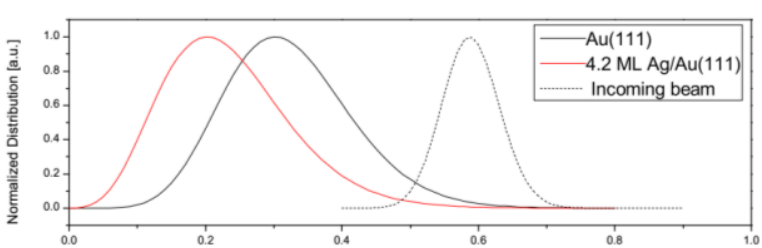

Kinetic Energy [eV]

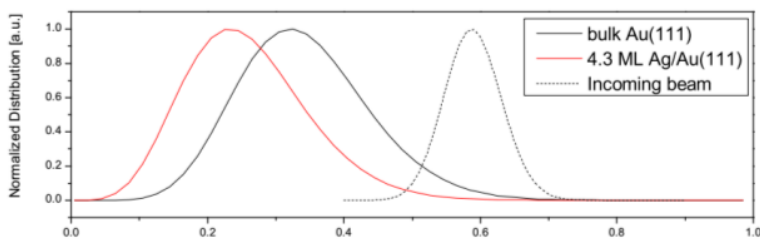

Kinetic Energy [eV]

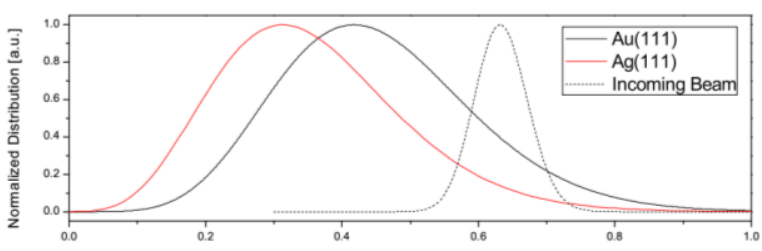

Kinetic Energy $[\mathrm{eV}]$

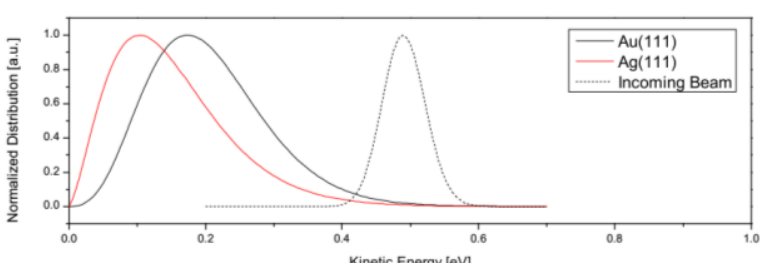

Figure 8.2: Compare scattering from Ag/Au films and bulk $\mathrm{Ag}(111)$ : Arrival time distributions (left panel) and kinetic energy distributions (right panel) for $\mathrm{NO}(v=2 \rightarrow 2)$ and $\mathrm{NO}(v=0 \rightarrow 0)$ scattered from Au and thin films of Ag/Au (this work) and previously acquired data for $\mathrm{NO}(\mathrm{v}=\mathrm{O} \rightarrow 0$ ) from bulk samples of Au(111) and Ag(111) (courtesy of Jascha Lau). For a common incidence energy the width of the final kinetic energy (right panel) that is independent of the scattering target. That is observed for the NO(v=O $\rightarrow 0$ ) scattered from bulk $\mathrm{Au}(111)$ and $\mathrm{Ag}(111)$ (plot 3,4 at the bottom) and both elastic scattering channels scattered from Au(111) and Ag/Au(111). If scattering from bulk and thin films exhibits the same scattering behavior, very good film quality is indicated. It even suggests that there is no difference between scattering from thin films of Ag/Au(111) and bulk Ag(111). 

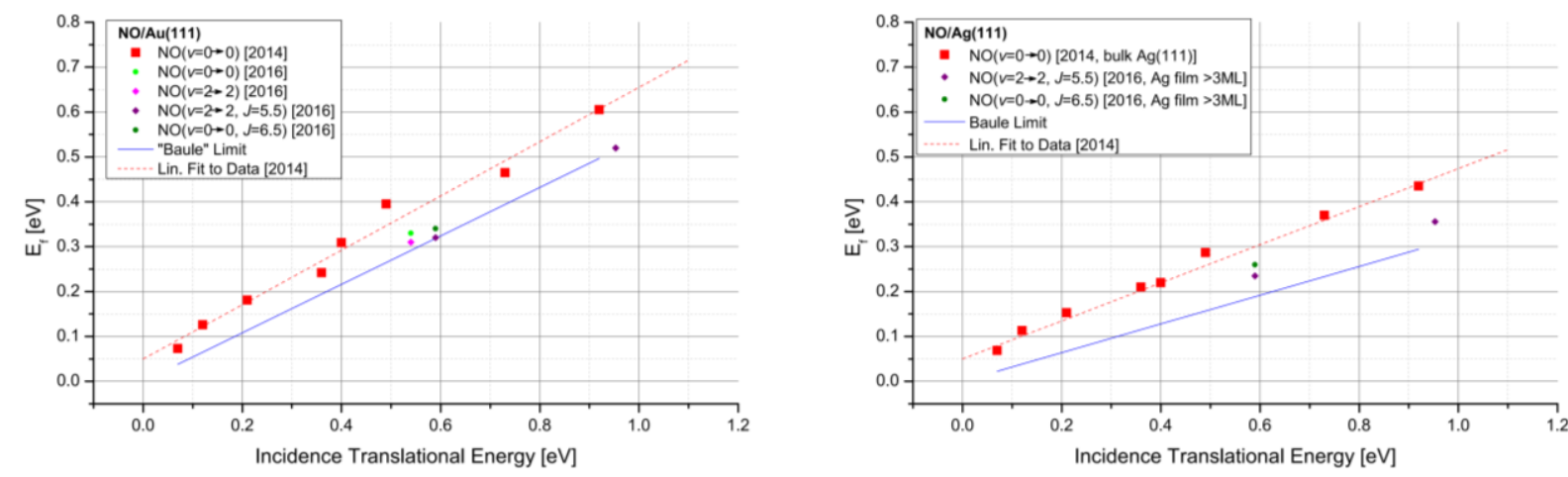

Figure 8.3: Final translational energy of NO after vibrationally elastic scattering on Au(111) and Ag(111). Comparison of $\mathrm{NO}(v=0 \rightarrow 0)$ scattered off of bulk $\mathrm{Au}(111)$ and bulk Ag(111) for a set of incidence energies (courtesy of Jascha Lau) with the data from this work. All data was acquired on the same machine. Within the error of the experiment, there is a small systematic offset between both data sets, probably due to differences in the flight length.

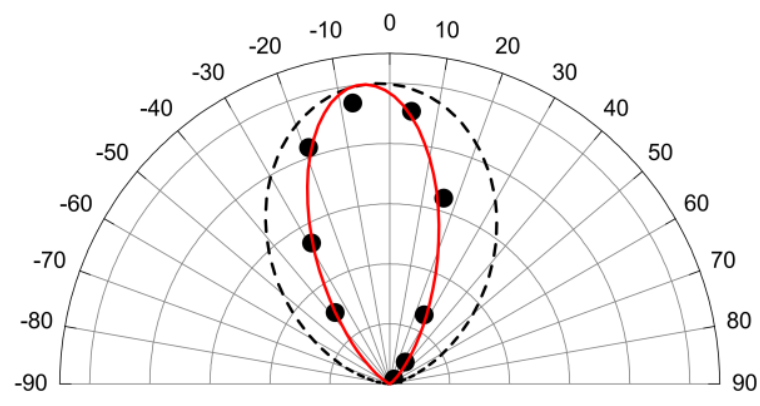

(a) $\mathrm{Au}(111)$

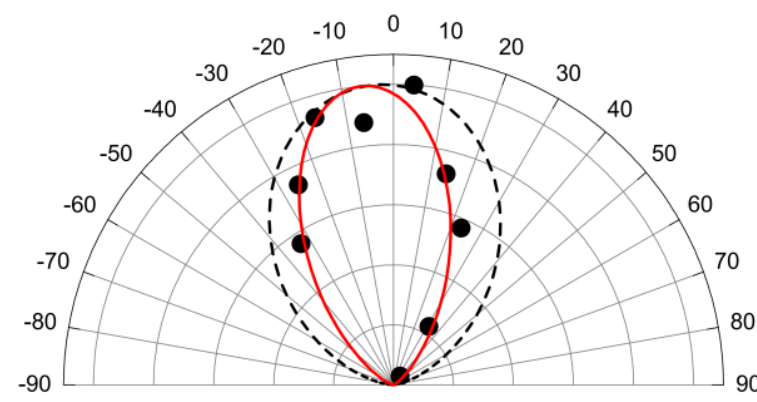

(b) $\operatorname{Ag}(111)$

Figure 8.4: Angular distributions of $\mathrm{NO}\left(\mathrm{v}=\mathrm{O} \rightarrow 0\right.$ ) scattering from bulk $\mathrm{Au}(111)$ and bulk $\mathrm{Ag}(111)$ for $E_{i}=0.92 \mathrm{eV}$ (courtesy of Sascha Lau). The solid line shows a fitting function of $\cos ^{n}\left(\vartheta+\vartheta_{0}\right)$. Parameters for $A u(111): n=7.36, \vartheta_{0}=5.42$. Parameters for $n=5.42, \vartheta_{0}=6.12$. Scattering from $A g(111)$ shows a slightly broader distribution in comparison with $\mathrm{Au}(111)$, a similar effect as observed for vibrationally elastic scattering from Au(111) and thin films of Ag/Au(111) (see Figure 5.6). 
D Data $\mathrm{CO}(v=0 \rightarrow 0)$ on $\mathrm{Au}(111)$ and $\mathrm{Ag}(111)$

\section{TOF Shapes for low rotational states}
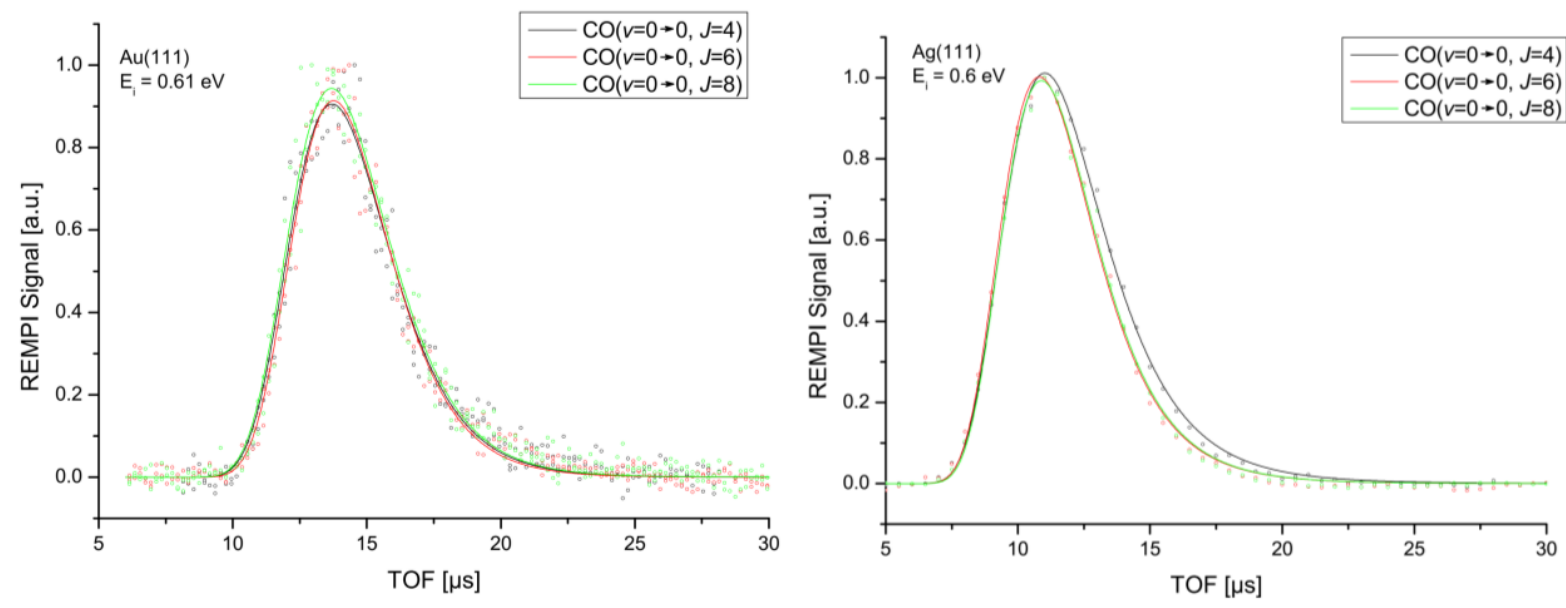

Figure $8.5 \mathrm{CO}(v=0 \rightarrow 0)$ TOF Shapes for low J states after scattering from Au(111) and Ag(111) (Courtesy of Pranav Shirhatti). The shapes of low J states are indistinguishable and can therefore be seen as a good approximation for the case of near elastic rotational scattering.

\section{Mean final kinetic energy vs rotational energy}
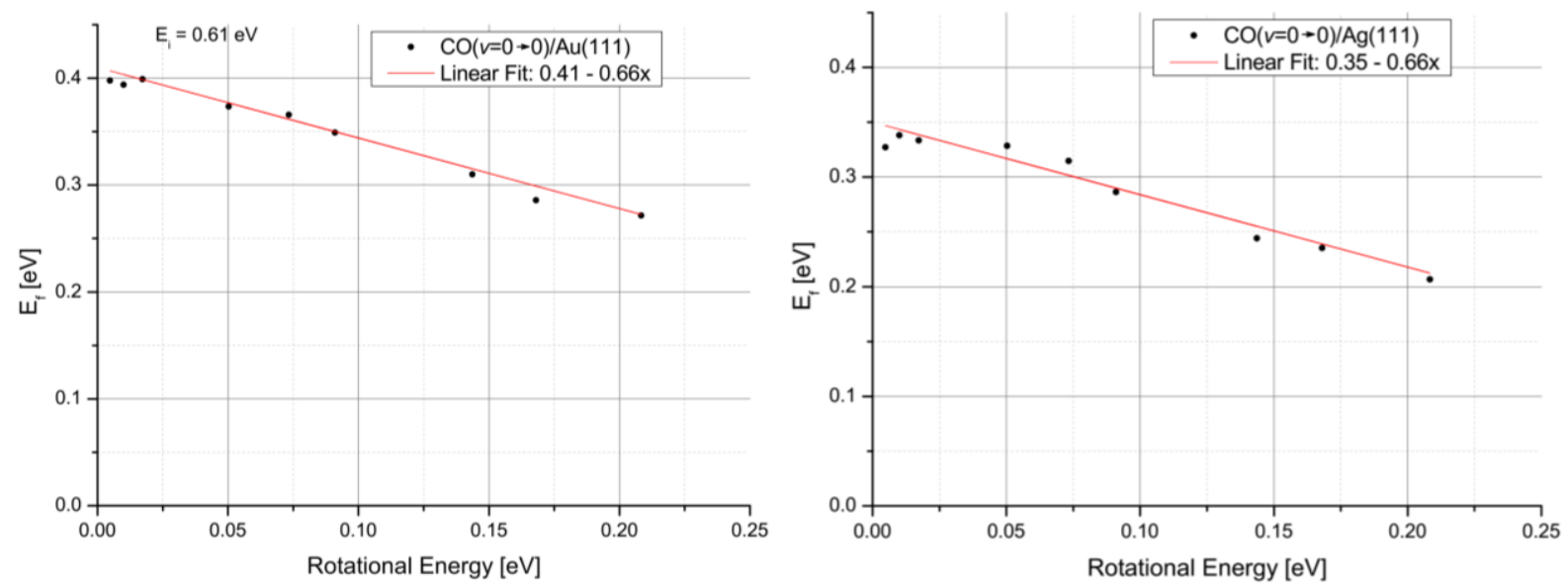

Figure 8.6: $\mathrm{CO}(v=0 \rightarrow 0)$ scattering from bulk $A u(111)$ and $A g(111)$. The mean final translational energy is detected for a number of rotational states and plotted against the rotational energy. The incidence energies used are close to the ones employed in this work. 


\section{E Parameters for the model potential energy surface of $\mathrm{NO} / \mathrm{Au}(111)$ and similar systems}

This part of the appendix is about the model invoked to explain the amount of vibrational relaxation of a gas-molecule system. Apart from a short introduction for the original NO/Au(111) system, it contains the documentation about the parameters that have been adjusted to describe $\mathrm{NO} / \mathrm{Ag} / \mathrm{Au}(111), \mathrm{CO} / \mathrm{Au}(111)$ and $\mathrm{CO} / \mathrm{Ag} / \mathrm{Au}(111)$.

The model employed to discuss the experimental results is strongly related to a potential energy surface of NO/Au(111) developed by Sharani Roy, Neil Shenvi and John Tully [98]. This PES is based on $a b$ initio calculations by density-functional theory using the Vienna $a b$ initio simulation package (VASP) software in the plane-wave basis. Ion-electron interaction was described by the PAW method and for the exchange-correlation, the Perdew-Wang 91 (PW91) functional was used within the generalizedgradient approximation (GGA). The calculated points (for different molecule-surface distances, NO bond lengths and orientations) were fitted by a functional form yielding an analytic representation of the ground states PES.

The diabatic description includes the neutral and anionic electronic states of the NO molecule interacting with the $\mathrm{Au}(111)$ surface and an off-diagonal coupling term that mixes the two diabatic configurations. The electronic Hamiltonian for a fixed nuclear configuration is constructed as the $(2 \times 2)$ Hamiltonian matrix

$$
H=\left[\begin{array}{ll}
H_{00} & H_{01} \\
H_{01} & H_{11}
\end{array}\right]
$$

where the diagonal element $H_{00}$ is the diabatic PES for the interaction of the neutral (uncharged) NO with $\mathrm{Au}(111), H_{11}$ is the diabatic PES describing the interaction if the ionic NO with $\mathrm{Au}(111)$ and $H_{01}$ is the off-diagonal coupling term. All terms of the matrix are dependent on the $\mathrm{N}, \mathrm{O}$ and $\mathrm{Au}$ positions.

In the following explication, it will be concentrated on the terms which have been adjusted to obtain the contour plots that are used in the discussion in sections 5.2 and 6.2 which are $H_{00}$ and $H_{11} . H_{01}$ is kept unchanged according to the original values.

The interaction potential of the neutral NO with $\mathrm{Au}(111) H_{00}$ consists of the two parts (eq. 8.12). One part describes the interaction between the ith of the $n$ Au atoms at position $\boldsymbol{r}_{i}$ with the $\mathrm{N}$ and $\mathrm{O}$ atom of the molecule and the other part describes the the $\mathrm{N}-\mathrm{O}$ interaction.

$$
H_{00}=\sum_{i=1}^{n}\left[V_{00}^{\mathrm{Au}-\mathrm{O}}\left(\left|\boldsymbol{r}_{i}-\boldsymbol{r}_{\mathrm{O}}\right|\right)+V_{00}^{\mathrm{Au}-\mathrm{N}}\left(\left|\boldsymbol{r}_{i}-\boldsymbol{r}_{\mathrm{N}}\right|\right)\right]+V_{00}^{\mathrm{N}-\mathrm{O}}\left(\left|\boldsymbol{r}_{\mathrm{N}}-\boldsymbol{r}_{\mathrm{O}}\right|\right)
$$

The second term, the $\mathrm{N}-\mathrm{O}$ interaction, is modeled by a Morse potential with the parameters $F_{0}, \gamma_{0}$ and $\boldsymbol{r}_{0}^{\mathrm{N}-\mathrm{O}}$.

$$
V_{00}^{\mathrm{N}-\mathrm{O}}\left(\left|\boldsymbol{r}_{\mathrm{N}}-\boldsymbol{r}_{\mathrm{O}}\right|\right)=F_{0}\left(1-e^{-\gamma_{0}\left(\left|\boldsymbol{r}_{\mathrm{N}}-\boldsymbol{r}_{\mathrm{O}}\right|-\boldsymbol{r}_{0}^{\mathrm{N}-0}\right)}\right)^{2}
$$

The second diabatic interaction potential of the anionic $\mathrm{NO}^{-}$and $\mathrm{Au}(111) H_{11}$ is defined as:

$$
H_{11}=V_{11}^{\mathrm{image}}+\sum_{i=1}^{n}\left[V_{11}^{\mathrm{Au}-\mathrm{O}}\left(\left|\boldsymbol{r}_{i}-\boldsymbol{r}_{\mathrm{O}}\right|\right)+V_{11}^{\mathrm{Au}-\mathrm{N}}\left(\left|\boldsymbol{r}_{i}-\boldsymbol{r}_{\mathrm{N}}\right|\right)\right]+V_{11}^{\mathrm{N}-\mathrm{O}}\left(\left|\boldsymbol{r}_{\mathrm{N}}-\boldsymbol{r}_{\mathrm{O}}\right|\right)+\phi-E_{a}
$$


Apart from the interactions analogous to neutral potential (eq. 8.12), a term $V_{11}^{\text {image }}$ describing the image charge stabilization, the work function $\phi$ and the electron affinity $E_{a}$ are additionally included. Especially the last two parameters are important when the model is used for other surfaces or molecules. The $\mathrm{N}-\mathrm{O}$ interaction of the anionic potential is described by a Morse potential, analogously to (8.13):

$$
V_{11}^{\mathrm{N}-\mathrm{O}}\left(\left|\boldsymbol{r}_{\mathrm{N}}-\boldsymbol{r}_{\mathrm{O}}\right|\right)=F_{1}\left(1-e^{-\gamma_{1}\left(\left|\boldsymbol{r}_{\mathrm{N}}-\boldsymbol{r}_{\mathrm{O}}\right|-\boldsymbol{r}_{1}^{\mathrm{N}-\mathrm{O}}\right)}\right)^{2}
$$

The adjusted values of the model are summarized in Table. The smallest adjustments are carryout out when changing from the surface $\mathrm{Au}$ to $\mathrm{Ag} / \mathrm{Au}(111)$. It is assumed that the interactions $V_{00,11}^{\mathrm{Ag}-\mathrm{N}}, V_{00,11}^{\mathrm{Ag}-\mathrm{O}}$ do not change significantly in comparison to a $\mathrm{Au}(111)$ surface, only the work function is significantly different. This seems justified as the authors of the model point out that the "neutral $H_{00}$ and ionic $H_{11}$ energies were fit to interaction potentials closely resembling those used in previous MD simulations of $\mathrm{NO}$ molecules scattering from $\mathrm{Ag}(111)$ and $\mathrm{Pt}(111)$ surfaces" [98], therefore it is assumed that $\mathrm{Ag} / \mathrm{Au}(111)$ films can be described in the same manner.

When discussing the system for $\mathrm{CO}$, the molecular parameters of this molecule were adjusted according to Reference [102]. However, the biggest changes arise due to the change of the electron affinity $E_{a}$.

Table 2: Parameters used in the model of the potential energy surface.

\begin{tabular}{|c|c|c|c|c|}
\hline & NO on $\mathrm{Au}(\mathbf{1 1 1})$ & $\mathrm{NO}$ on $\mathrm{Ag} / \mathrm{Au}(111)$ & $\mathrm{CO}$ on $\mathrm{Au}(111)$ & $\mathrm{CO}$ on $\mathrm{Ag} / \mathrm{Au}(111)$ \\
\hline \multicolumn{5}{|c|}{ Neutral Diabat } \\
\hline$F_{0}[\mathrm{eV}]$ & 6.53571 & 6.53571 & 11.09 & 11.09 \\
\hline$\gamma_{0}[1 / \AA ̊ \AA]$ & 2.743 & 2.743 & 2.3146 & 2.3146 \\
\hline $\boldsymbol{r}_{0}^{\mathrm{N}-\mathrm{O}}[\AA]$ & 1.15077 & 1.15077 & 1.1315 & 1.1315 \\
\hline \multicolumn{5}{|c|}{ Ionic Diabat } \\
\hline$F_{1}[\mathrm{eV}]$ & 5.14047 & 5.14047 & 8.1 & 8.1 \\
\hline$\gamma_{1}[1 / \AA ̊ A]$ & 2.489 & 2.489 & 2.46081 & 2.46081 \\
\hline $\boldsymbol{r}_{1}^{\mathrm{N}-\mathrm{O}}[\AA]$ & 1.2904 & 1.2904 & 1.17 & 1.17 \\
\hline$\phi[\mathrm{eV}]$ & 5.3 & $4.7[4.9]$ & 5.3 & 4.7 \\
\hline$E_{a}[\mathrm{eV}]$ & -0.007 & -0.007 & -1.5 & -1.5 \\
\hline $\begin{array}{l}\text { Parameters } \\
\text { used in... }\end{array}$ & $\begin{array}{l}\text { Figure 5.20, } \\
\text { Figure 5.24, } \\
\text { Figure } 6.17 \text { (left) } \\
\end{array}$ & $\begin{array}{l}\text { Figure 5.21, } \\
\text { Figure } 5.24 \text { (right) } \\
\text { [Figure } 5.24 \text { (center)] }\end{array}$ & $\begin{array}{l}\text { Figure } 5.25 \text { (left), } \\
\text { Figure } 6.17 \text { (right) }\end{array}$ & Figure 5.25 (right) \\
\hline
\end{tabular}




\section{References}

[1] G. Ertl, Reaktionen an Oberflächen: vom Atomaren zum Komplexen (Nobel-Vortrag), Angew. Chemie 2008, 120, 3578-3590.

[2] C.T. Rettner, D.J. Auerbach, J.C. Tully, A.W. Kleyn, Chemical Dynamics at the Gas-Surface Interface, J. Phys. Chem. 1996, 100, 13021-13033.

[3] J.E. Hurst, C.A. Becker, J.P. Cowin, K.C. Janda, L. Wharton, D.J. Auerbach, Observation of direct inelastic scattering in the presence of trapping-desorption scattering: Xe on Pt(111), Phys. Rev. Lett. 1979, 43, 1175-1177.

[4] J.E. Hurst, L. Wharton, K.C. Janda, D.J. Auerbach, Direct inelastic scattering Ar from Pt(111), J. Chem. Phys. 1983, 78, 1559-1581.

[5] A.W. Kleyn, A.C. Luntz, D.J. Auerbach, Rotational energy transfer in direct inelastic surface scattering: NO on Ag(111), Phys. Rev. Lett. 1981, 47, 1169-1172.

[6] C.T. Rettner, J. Kimman, D.J. Auerbach, Inelastic scattering of NO from Ag(111): Internal state, angle, and velocity resolved measurements, J. Chem. Phys. 1991, 94, 734.

[7] C.T. Rettner, The search for direct vibrational excitation in gas-surface collisions of CO with Au(111), J. Chem. Phys. 1993, 99, 5481-5489.

[8] G. Ertl, Elementary Steps in Heterogeneous Catalysis, Angew. Chemie Int. Ed. English 1990, 29, 1219-1227.

[9] C. Argile, G.E. Rhead, Adsorbed layer and thin film growth modes monitored by Auger electron spectroscopy, Surf. Sci. Rep. 1989, 10, 277-356.

[10] F. Forster, E. Gergert, A. Nuber, H. Bentmann, L. Huang, X.G. Gong, Z. Zhang, F. Reinert, Electronic localization of quantum-well states in $\mathrm{Ag} / \mathrm{Au}(111)$ metallic heterostructures, Phys. Rev. B - Condens. Matter Mater. Phys. 2011, 84, 1-8.

[11] C. Reuß, W. Wallauer, T. Fauster, Image States of Ag on Au(111), Surf. Rev. Lett. 1996, 3, 15471554.

[12] H. Cercellier, C. Didiot, Y. Fagot-Revurat, B. Kierren, L. Moreau, D. Malterre, F. Reinert, Interplay between structural, chemical, and spectroscopic properties of $A g / A u(111)$ epitaxial ultrathin films: A way to tune the Rashba coupling, Phys. Rev. B - Condens. Matter Mater. Phys. 2006, 73, $1-16$.

[13] J.A. Rodriguez, D.W. Goodman, The nature of the metal-metal bond in bimetallic surfaces., Science 1992, 257, 897-903.

[14] B.C. Krüger, N. Bartels, C. Bartels, A. Kandratsenka, J.C. Tully, A.M. Wodtke, T. Schäfer, NO Vibrational Energy Transfer on a Metal Surface: Still a Challenge to First-Principles Theory, J. Phys. Chem. C 2015, 119, 3268-3272.

[15] A.M. Wodtke, J.C. Tully, D.J. Auerbach, Electronically non-adiabatic interactions of molecules at metal surfaces: Can we trust the Born-Oppenheimer approximation for surface chemistry?, Int. Rev. Phys. Chem. 2004, 23, 513-539.

[16] B.I. Lundqvist, A. Hellman, I. Zorić, Chapter 10 Electron Transfer and Nonadiabaticity, Handb. Surf. Sci. 2008, 3, 429-524. 
[17] E. Hasselbrink, How non-adiabatic are surface dynamical processes?, Curr. Opin. Solid State Mater. Sci. 2006, 10, 192-204.

[18] I. Rahinov, R. Cooper, D. Matsiev, C. Bartels, D.J. Auerbach, A.M. Wodtke, Quantifying the breakdown of the Born-Oppenheimer approximation in surface chemistry., Phys. Chem. Chem. Phys. 2011, 13, 12680-12692.

[19] M. Born, R. Oppenheimer, Zur Quantentheorie der Molekeln, Ann. Phys. 1927, 389, 457-484.

[20] B. McCarroll, Chemisorptive Luminescence, J. Chem. Phys. 1969, 50, 4758-4765.

[21] T. Greber, R. Grobecker, A. Morgante, A. Böttcher, G. Ertl, O-Escape during the Oxidation of Cesium, Phys. Rev. Lett. 1993, 70, 1331-1334.

[22] L.D. Trowbridge, D.R. Herschbach, Summary Abstract: Surface chemi-ionization on alkali metals, J. Vac. Sci. Technol. 1981, 18, 588-589.

[23] M. Morin, N.J. Levinos, A.L. Harris, Vibrational energy transfer of $\mathrm{CO} / \mathrm{Cu}(100)$ : Nonadiabatic vibration/electron coupling, J. Chem. Phys. 1992, 96, 3950-3956.

[24] H.C. Chang, G.E. Ewing, Infrared fluorescence from a monolayer of $\mathrm{CO}$ on $\mathrm{NaCl}(100)$, Phys. Rev. Lett. 1990, 65, 2125-2128.

[25] B. Gergen, H. Nienhaus, W.H. Weinberg, E.W. McFarland, Chemically induced electronic excitations at metal surfaces., Science 2001, 294, 2521-2523.

[26] D. Diesing, E. Hasselbrink, Chemical energy dissipation at surfaces under UHV and high pressure conditions studied using metal-insulator-metal and similar devices, Chem. Soc. Rev. 2016, 45, 3747-3755.

[27] O. Bünermann, H. Jiang, Y. Dorenkamp, A. Kandratsenka, S.M. Janke, D.J. Auerbach, A.M. Wodtke, Electron-hole pair excitation determines the mechanism of hydrogen atom adsorption, Science 2015, 350, 1346-1349.

[28] R. Cooper, Z. Li, K. Golibrzuch, C. Bartels, I. Rahinov, D.J. Auerbach, A.M. Wodtke, On the determination of absolute vibrational excitation probabilities in molecule-surface scattering: Case study of NO on Au(111), J. Chem. Phys. 2012, 137,.

[29] T. Schäfer, N. Bartels, K. Golibrzuch, C. Bartels, H. Köckert, D.J. Auerbach, T.N. Kitsopoulos, A.M. Wodtke, Observation of direct vibrational excitation in gas-surface collisions of CO with Au(111): a new model system for surface dynamics., Phys. Chem. Chem. Phys. 2013, 15, 1863-7.

[30] C.T. Rettner, F. Fabre, J. Kimman, D.J. Auerbach, Observation of direct vibrational excitation in gas-surface collisions: NO on ag(111), Phys. Rev. Lett. 1985, 55, 1904-1907.

[31] D.M. Newns, Electron-hole pair mechanism for excitation of intramolecular vibrations in molecule-surface scattering, Surf. Sci. 1986, 171, 600-614.

[32] B.D. Kay, T.D. Raymond, M.E. Coltrin, Observation of direct multiquantum vibrational excitation in gas-surface scattering: NH3 on Au(111), Phys. Rev. Lett. 1987, 59, 2792-2794.

[33] Q. Ran, D. Matsiev, D.J. Auerbach, A.M. Wodtke, Observation of a change of vibrational excitation mechanism with surface temperature: $\mathrm{HCl}$ collisions with $\mathrm{Au}(111)$, Phys. Rev. Lett. 2007, 98,

[34] E.K. Watts, J.L.W. Siders, G.O. Sitz, Vibrational excitation of NO scattered from Cu(110), Surf. Sci. 1997, 374, 191-196. 
[35] R. Cooper, C. Bartels, A. Kandratsenka, I. Rahinov, N. Shenvi, K. Golibrzuch, Z. Li, D.J. Auerbach, J.C. Tully, A.M. Wodtke, Multiquantum vibrational excitation of NO scattered from Au(111): Quantitative comparison of benchmark data to Ab initio theories of nonadiabatic moleculesurface interactions, Angew. Chemie - Int. Ed. 2012, 51, 4954-4958.

[36] P.O. Gartland, S. Berge, B.J. Slagsvold, Photoelectric work function of a copper single crystal for the (100), (110), (111), and (112) faces, Phys. Rev. Lett. 1972, 28, 738-739.

[37] M. Chelvayohan, C.H.B. Mee, Work function measurements on (110), (100) and (111) surfaces of silver, J. Phys. C Solid State Phys. 1982, 15, 2305-2312.

[38] G. Hansson, S. Flodström, Photoemission study of the bulk and surface electronic structure of single crystals of gold, Phys. Rev. B 1978, 18, 1572-1585.

[39] J. Werdecker, P.R. Shirhatti, K. Golibrzuch, C. Bartels, A.M. Wodtke, D.J. Harding, Electronically Nonadiabatic Vibrational Excitation of N2 Scattered from Pt(111), J. Phys. Chem. C 2015, 119, 14722-14727.

[40] P.R. Shirhatti, J. Werdecker, K. Golibrzuch, A.M. Wodtke, C. Bartels, Electron hole pair mediated vibrational excitation in $\mathrm{CO}$ scattering from $\mathrm{Au}(111)$ : Incidence energy and surface temperature dependence, J. Chem. Phys. 2014, 141,.

[41] J. Geweke, P.R. Shirhatti, I. Rahinov, C. Bartels, A.M. Wodtke, Vibrational energy transfer near a dissociative adsorption transition state: State-to-state study of $\mathrm{HCl}$ collisions at $\mathrm{Au(111),} \mathrm{J.}$ Chem. Phys. 2016, 145,.

[42] P.R. Shirhatti, J. Geweke, C. Steinsiek, C. Bartels, I. Rahinov, D.J. Auerbach, A.M. Wodtke, Activated Dissociation of $\mathrm{HCl}$ on $\mathrm{Au}(111)$, J. Phys. Chem. Lett. 2016, 7, 1346-1350.

[43] Y. Huang, C.T. Rettner, D.J. Auerbach, A.M. Wodtke, Vibrational Promotion of Electron Transfer, Science 2000, 290, 111-114.

[44] J.D. White, J. Chen, D. Matsiev, D.J. Auerbach, A.M. Wodtke, Conversion of large-amplitude vibration to electron excitation at a metal surface, Nature 2005, 433, 503-505.

[45] Y. Huang, A.M. Wodtke, H. Hou, C.T. Rettner, D.J. Auerbach, Observation of Vibrational Excitation and Deexcitation for $N O(v=2)$ Scattering from $A u$ (111): Evidence for Electron-HolePair Mediated Energy Transfer, Phys. Rev. Lett. 2000, 84, 2985-2988.

[46] N. Bartels, B.C. Krüger, D.J. Auerbach, A.M. Wodtke, T. Schäfer, Controlling an electron-transfer reaction at a metal surface by manipulating reactant motion and orientation, Angew. Chemie Int. Ed. 2014, 53, 13690-13694.

[47] N. Shenvi, S. Roy, J.C. Tully, Dynamical steering and electronic excitation in NO scattering from a gold surface, Science 2009, 326, 829-832.

[48] B.C. Krüger, S. Meyer, A. Kandratsenka, A.M. Wodtke, T. Schäfer, Vibrational Inelasticity of Highly Vibrationally Excited NO on Ag(111), J. Phys. Chem. Lett. 2016, 7, 441-446.

[49] K. Golibrzuch, Quantum-state specific scattering of molecules from surfaces, 2014.

[50] H. Lüth, Solid Surfaces, Interfaces and Thin Films, 3., Springer Verlag, Berlin Heidelberg NewYork, 2001.

[51] D.C. Jackson, T.E. Gallon, A. Chambers, A model for the Auger electron spectroscopy of systems exhibiting layer growth, and its application to the deposition of silver on nickel, Surf. Sci. 1973, 36, 381-394. 
[52] W.P. Davey, Precision measurements of the lattice constants of twelve common metals, Phys. Rev. 1925, 25, 753-761.

[53] P.W. Palmberg, T.N. Rhodin, Auger electron spectroscopy of fcc metal surfaces, J. Appl. Phys. $1968,39,2425-2432$.

[54] H. Tokutaka, K. Nishimori, K. Takashima, A QUANTITATIVE AES METHOD FOR THE STUDY OF A MONOLAYER OVER-GROWTH OF THIN FILM, Surf. Sci. 1979, 86, 54-61.

[55] M.M. Dovek, C.A. Lang, J. Nogami, C.F. Quate, Epitaxial growth of Ag on Au(111) studied by scanning tunneling microscopy, Phys. Rev. B 1989, 40, 11973-11975.

[56] D.D. Chambliss, R.J. Wilson, Relaxed diffusion limited aggregation of Ag on Au(111) observed by scanning tunneling microscopy, J. Vac. Sci. Technol. B Microelectron. Nanom. Struct. 1991, 9, 928.

[57] H. Meinel, K.; Klaua, M.; Bethge, TEM AND AES INVESTIGATIONS OF SURFACE-DIFFUSIONINDUCED AGING PROCESSES IN THIN Au FILMS ON Ag(111), Ultramicroscopy 1986, 20, 261-267.

[58] K. Besocke, H. Wagner, Adsorption of W on W(110): Work-Function Reduction and Island Formation, Phys. Rev. B 1973, 8, 4597.

[59] M. Alnot, J.J. Ehrhardt, J.A. Barnard, A characterization of heterogeneous Pt surfaces by work function measurements and photoemission of adsorbed xenon, Surf. Sci. 1989, 208, 285-305.

[60] W. Shockley, On the surface states associated with a periodic potential, Phys. Rev. 1939, 56, 317-323.

[61] I. Tamm, Über eine mögliche Art der Elektronenbindung an Kristalloberflächen, Z. Phys 1932, 76, 849-850.

[62] P.O. Gartland, B.J. Slagsvold, Transitions conserving parallel momentum in photoemission from the (111) face of copper, Phys. Rev. B 1975, 12, 4047-4058.

[63] T.C. Chiang, Photoemission studies of quantum well states in thin films, Surf. Sci. Rep. 2000, 39, 181-235.

[64] B.J. Hinch, C. Koziol, J.P. Toennies, G. Zhang, Evidence for Quantum Size Effects Observed by Helium Atom Scattering during the Growth of Pb on Cu(111), Europhys. Lett. 1989, 10, 341-346.

[65] J. Paggel, C. Wei, M. Chou, D. -a. Luh, T. Miller, T.-C. Chiang, Atomic-layer-resolved quantum oscillations in the work function: Theory and experiment for $\mathrm{Ag} / \mathrm{Fe}(100)$, Phys. Rev. B 2002, $66,1-4$.

[66] P.S. Kirchmann, M. Wolf, J.H. Dil, K. Horn, U. Bovensiepen, Quantum size effects in Pb Si (111) investigated by laser-induced photoemission, Phys. Rev. B - Condens. Matter Mater. Phys. 2007, 76, 5-9.

[67] M.N. Baibich, J.M. Broto, A. Fert, F. Nguyen Van Dau, F. Petroff, P. Eitenne, G. Creuzet, A. Friederich, J. Chazelas, Giant Magnetorestistance of (001)Fe/(001)Cr Magnetic Superlattices, Phys. Rev. Lett. 1988, 61, 2472-2475.

[68] X. Ma, P. Jiang, Y. Qi, J. Jia, Y.Y. Yang, W. Duan, W.-X.W.-X.W.-X. Li, X. Bao, S.B. Zhang, Q.-K. Xue, Experimental observation of quantum oscillation of surface chemical reactivities, Proc. Natl. Acad. Sci. 2007, 104, 9204-9208.

[69] U. Hagemann, $\mathrm{H}$. Nienhaus, Quantum size effects in chemicurrent measurements during lowtemperature oxidation of Mg(0001) epilayers, New J. Phys. 2014, 16, 1-14. 
[70] T. Miller, A. Samsavar, G.E. Franklin, T.C. Chiang, Quantum-well states in a metallic system: Ag on Au(111), Phys. Rev. Lett. 1988, 61, 1404-1407.

[71] C.T. Campbell, Bimetallic Model Catalysts, in: G. Ertl, H. Knozinger, J. Weitkamp (Eds.), Handb. Heterog. Catal., Wiley-VCH, Weinheim, 1997: pp. 814-826.

[72] J.A. Rodriguez, R.A. Campbell, D.W. Goodman, Electron donor--electron acceptor interactions in surface metal--metal bonds: The $\mathrm{Cu} / \mathrm{Re}(0001)$ and $\mathrm{Pd} / \mathrm{Re}(0001)$ systems, 38th Natl. Symp. Am. Vac. Soc. 1992, 10, 2540-2545.

[73] J.A. Rodriguez, R.A. Campbell, D.W. Goodman, Electron donor-electron acceptor interactions in bimetallic surfaces: Theory and XPS studies, J. Phys. Chem. 1991, 95, 5716-5719.

[74] A.K. Santra, C.N.R. Rao, Interaction of Carbon Monoxide with Bimetallic Overlayers, J. Chem. Phys. 1994, 98, 5962-5965.

[75] A.R. Miedema, J.W.F. Dorleijn, Quantitative predictions of the heat of adsorption of metals on metallic substrates, Surf. Sci. 1980, 95, 447-464.

[76] A. Ruban, B. Hammer, P. Stoltze, H.L. Skriver, J.K. Nørskov, Surface electronic structure and reactivity of transition and noble metals, J. Mol. Catal. A Chem. 1997, 115, 421-429.

[77] B. Hammer, Y. Morikawa, J. Nørskov, CO Chemisorption at Metal Surfaces and Overlayers, Phys. Rev. Lett. 1996, 76, 2141-2144.

[78] G.Z. Sauerbrey, No Title, Phys. Verh. 1958, 8, 193.

[79] C.S. Lu, O. Lewis, Investigation of film-thickness determination by oscillating quartz resonators with large mass load, J. Appl. Phys. 1972, 43, 4385-4390.

[80] K. Meinel, M. Klaua, H. Bethge, The influence of the substrate microtopography on the growth of thin films studied for the Au-Ag system by TEM and AES, J. Cryst. Growth 1988, 89, 447-458.

[81] Q. Ran, D. Matsiev, A.M. Wodtke, D.J. Auerbach, An advanced molecule-surface scattering instrument for study of vibrational energy transfer in gas-solid collisions, Rev. Sci. Instrum. 2007, 78 .

[82] G. Scoles, Atomic and Molecular Beam Methods, 1988.

[83] R.K. Kawakami, E. Rotenberg, H.J. Choi, E.J. Escorcia-Aparicio, M.O. Bowen, J.H. Wolfe, E. Arenholz, Z.D. Zhang, N. V Smith, Z.Q. Qiu, Quantum-well states in copper thin films, Nature 1999, 398, 132-134.

[84] T. Uchihashi, T. Nakayama, Locality and lateral modulations of quantum well states in Ag(100) thin films studied using a scanning tunneling microscope, Surf. Sci. 2015, 637-638, 58-62.

[85] J. Kimman, C.T. Rettner, D.J. Auerbach, J.A. Barker, J.C. Tully, Correlation between KineticEnergy Transfer to Rotation and to Phonons in Gas-Surface Collisions of NO with Ag(111), Phys. Rev. Lett. 1986, 57, 2053-2056.

[86] T. Hanisco, C. Yan, A. Kummel, Energy and momentum distributions versus incident energy in the scattering of CO from Ag(111), J. Vac. Sci. Technol. A Vacuum, Surfaces, Film. 1993, 11, 2090.

[87] K. Golibrzuch, P.R. Shirhatti, J. Altschäffel, I. Rahinov, D.J. Auerbach, A.M. Wodtke, C. Bartels, State-to-state time-of-flight measurements of NO scattering from Au(111): Direct observation of translation-to-vibration coupling in electronically nonadiabatic energy transfer, J. Phys. Chem. A 2013, 117, 8750-8760. 
[88] K. Golibrzuch, P.R. Shirhatti, I. Rahinov, D.J. Auerbach, A.M. Wodtke, C. Bartels, Incidence energy dependent state-to-state time-of-flight measurements of $\mathrm{NO}(\mathrm{v}=3)$ collisions with Au(111): the fate of incidence vibrational and translational energy., Phys. Chem. Chem. Phys. 2014, 16, 7602-10.

[89] I. Rahinov, R. Cooper, C. Yuan, X. Yang, D.J. Auerbach, A.M. Wodtke, Efficient vibrational and translational excitations of a solid metal surface: State-to-state time-of-flight measurements of $\mathrm{HCl}(v=2, J=1)$ scattering from Au(111), J. Chem. Phys. 2008, 129,.

[90] I.M. Lane, Z.P. Liu, D.A. King, H. Arnolds, Ultrafast vibrational dynamics of NO and CO adsorbed on an iridium surface, J. Phys. Chem. C 2007, 111, 14198-14206.

[91] H.A. Ory, A.P. Gittleman, J.P. Maddox, FRANCK-CONDON FACTORS FOR NO BETA + GAMMA BAND SYSTEMS, Astrophys. J. 1964, 139, 346.

[92] K. Golibrzuch, P.R. Shirhatti, I. Rahinov, A. Kandratsenka, D.J. Auerbach, A.M. Wodtke, C. Bartels, The importance of accurate adiabatic interaction potentials for the correct description of electronically nonadiabatic vibrational energy transfer: A combined experimental and theoretical study of $\mathrm{NO}(v=3)$ collisions with a Au(111) surface, J. Chem. Phys. 2014, 140,

[93] E.W. Kuipers, M.E.M. Spruit, A.W. Kleyn, DIFFERENTIAL TRAPPING PROBABILITIES AND DESORPTION OF PHYSISORBED MOLECULES : APPLICATION TO NO / Ag (111), Surf.Sci. 1988, 205, 241-268.

[94] J.D. White, J. Chen, D. Matsiev, D.J. Auerbach, A.M. Wodtke, Vibrationally promoted electron emission from low work-function metal surfaces, J. Chem. Phys. 2006, 124,

[95] A.W. Dweydari, C.H.B. Mee, Work function measurements on (100) and (110) surfaces of silver, Phys. Status Solidi 1975, 27, 223-230.

[96] N. Shenvi, S. Roy, J.C. Tully, Nonadiabatic dynamics at metal surfaces: Independent-electron surface hopping, J. Chem. Phys. 2009, 130,

[97] M.J. Travers, D.C. Cowles, G.B. Ellison, Reinvestigation of the electron affinities of 02 and NO, Chem. Phys. Lett. 1989, 164, 449-455.

[98] S. Roy, N.A. Shenvi, J.C. Tully, Model Hamiltonian for the interaction of NO with the Au(111) surface, J. Chem. Phys. 2009, 130,.

[99] D.P. Engelhart, R.J. V Wagner, A. Meling, A.M. Wodtke, T. Schäfer, Surface Science Temperature programmed desorption of weakly bound adsorbates on Au (111), Surf. Sci. 2016, 650, 11-16.

[100] P.A. Redhead, Thermal Desorption of Gases, Vacuum 1962, 12, 203-211.

[101] S.K. So, R. Franchy, W. Ho, The adsorption and reactions of NO on Ag (111) at $80 K, 1989,91$, 5701.

[102] I. Magoulas, A. Papakondylis, A. Mavridis, Structural parameters of the ground states of the quasi-stable diatomic anions $\mathrm{CO}-, \mathrm{BF}-$, and $\mathrm{BCl}$ - as obtained by conventional Ab Initio methods, Int. J. Quantum Chem. 2015, 115, 771-778.

[103] R.J. V Wagner, N. Henning, B.C. Krüger, G.B. Park, A.M. Wodtke, T. Schäfer, Scattering Highly Vibrationally Excited CO from Au(111): The Influence of Electron Affinity on Electronic NonAdiabaticity, in preparation, 2017.

[104] C.T. Rettner, H. a. Michelsen, D.J. Auerbach, Quantum-state-specific dynamics of the dissociative adsorption and associative desorption of $\mathrm{H} 2$ at a $\mathrm{Cu}(111)$ surface, J. Chem. Phys. 1995, 102, 4625. 
[105] N.S. Murthy, U.D. Prahllad, Determination of the transition moment of the $B 1 \Sigma+-X 1 \Sigma+$ transition in CO, J. Phys. B At. Mol. Phys. 1972, 5, 896-902.

[106] G.J. Schulz, Resonances in electron impact on diatomic molecules, Rev. Mod. Phys. 1973, 45, 423-486.

[107] J. Larsen, I. Chorkendorff, Increased dissociation probability of $\mathrm{CH}_{4}$ on $\mathrm{Co} / \mathrm{Cu}$ (111), Surf. Sci. 1998, 405, 62-73.

[108] W. Hansen, M. Bertolo, K. Jacobi, Physisorption of CO on Ag(111): investigation of the monolayer and the multilayer through HREELS, ARUPS, and TDS, Surf. Sci. 1991, 253, 1-12.

[109] C.T. Campbell, Bimetallic Surface Chemistry, Annu. Rev. Phys. Chem. 1990, 41, 775-837.

[110] J.A. Dean, Lange's handbook of chemistry, Fifteen Ed, McGraw-Hill Inc, 1999.

[111] R.E. Watson, J. Hudis, M.L. Perlman, Charge flow and d compensation in gold alloys, Phys. Rev. B 1971, 4, 4139-4144.

[112] M. Valden, X. Lai, D.W. Goodman, Onset of Catalytic Activity of Gold Clusters on Titania with the Appearance of Nonmetallic Properties, Science 1998, 281, 2-6.

[113] A.C. Luntz, Simple model for associative desorption and dissociative chemisorption, J. Chem. Phys. 2000, 113, 6901-6905. 


\section{Lebenslauf}

Persönliche Daten:

Diplom-Chemiker Christoph Steinsiek

Mittelstraße 3H

37077 Göttingen

Geburtsdatum: $\quad$ 16.11.1985

Geburtsort: Rheda-Wiedenbrück

Staatsangehörigkeit: deutsch

Familienstatus ledig

\section{Schulausbildung:}

09/1992 - 07/1996 Eichendorff-Schule Wiedenbrück (Grundschule)

09/1996-06/2005 Ratsgymnasium Wiedenbrück

Abschluss: Abitur (Note 1,6)

Universitäre Ausbildung:

$10 / 2005$

Beginn des Studiums an der Georg-August-Universität Göttingen

Fach: Chemie (Diplom)

04/2009-09/2009 Auslandspraktikum im Labor von Dr. Matthew Gaunt an der University of Cambridge

09/1010 - 03/2011 Diplomarbeit bei Prof. Dr. Alec Wodtke

Thema: „Time-Correlated Single Photon Counting on Quantum Well States“

$05 / 2011$

Seit 06/2011 Abschluss: Diplom (Gesamtnote: sehr gut)

Promotion an der Georg-August-Universität bei Prof. Dr. Alec Wodtke

Thema: „Molecular Beam Scattering from Ultrathin Metallic Films“

Weitere Ausbildung:

10/2011 - 09/2013 Teilnahme am Fernstudiengang "Lasertechnologie" an der Friedrich-SchillerUniversität Jena bei Doz. Dr. G. Staupendahl

\section{Sonstiges:}

04/2007 - 05/2011 Stipendiat des Evangelischen Studienwerks Villigst e.V.

01/2008-05/2011 Stipendiat der Studienstiftung des deutschen Volkes 